University of Louisville

ThinkIR: The University of Louisville's Institutional Repository

Electronic Theses and Dissertations

7-2015

\title{
Model of a tubular perfusion bioreactor using computational fluid dynamics.
}

Matthew James Robeson 1991-

University of Louisville

Follow this and additional works at: https://ir.library.louisville.edu/etd

Part of the Chemical Engineering Commons

\section{Recommended Citation}

Robeson, Matthew James 1991-, "Model of a tubular perfusion bioreactor using computational fluid dynamics." (2015). Electronic Theses and Dissertations. Paper 1996.

https://doi.org/10.18297/etd/1996

This Master's Thesis is brought to you for free and open access by ThinkIR: The University of Louisville's Institutional Repository. It has been accepted for inclusion in Electronic Theses and Dissertations by an authorized administrator of ThinkIR: The University of Louisville's Institutional Repository. This title appears here courtesy of the author, who has retained all other copyrights. For more information, please contact thinkir@louisville.edu. 
MODEL OF A TUBULAR PERFUSION BIOREACTOR USING COMPUTATIONAL FLUID DYNAMICS

\author{
By \\ Matthew James Robeson \\ B.S., University of Louisville, 2014 \\ A Thesis \\ Submitted to the Faculty of the \\ University of Louisville \\ In Partial Fulfillment of the Requirements \\ For the Professional Degree
}

MASTER OF ENGINEERING

Department of Chemical Engineering

July 2015 


\section{MODEL OF A TUBULAR PERFUSION BIOREACTOR}

USING COMPUTATIONAL FLUID DYNAMICS

Submitted by:

Matthew James Robeson

A Thesis Approved On

A Thesis Approved On
(Date)

By the Following Reading and Examination Committee:

Dr. R. Eric Berson, Co-Advisor

Dr. Gerold Willing

Dr. Martin O’Toole 


\section{ACKNOLWEDGEMENTS}

I would first like to acknowledge my mentors, Dr. Eric Berson, Dr. Eric Brey, and Jarel Gandhi for their immense help on this project. Without the support and guidance that they provided, this research never would have come to fruition. For their knowledge and patience over the last two years, I am deeply grateful.

This project began as part of a summer internship with the NSF Research Experience for Undergraduates project. The REU program not only gave me this project, but helped me to grow as a researcher and encouraged me to pursue graduate school. The support of the NSF and Illinois Institute of Technology made this work possible.

I would like to thank the members of my committee, Dr. Gerold Willing and Dr. Martin O'Toole, for agreeing to share their knowledge and critique with me, particularly on such short notice.

Lastly, I would like to thank my family and friends who kept my chin up through the good times and the bad. The importance of moral support should never be underestimated. 


\begin{abstract}
Through recent years, many novel bioreactor designs for tissue engineering have emerged. The Tubular Perfusion System (TPS) is one such bioreactor for bone and cartilage tissue engineering. The TPS is a perfusion type bioreactor, in which media flows through a bed of scaffold beads. The advantage of this system over similar designs is that it requires no special fabrication techniques and operates at low pressure. Experimental trials have shown increased osteoblastic differentiation and mineralization of bone tissue grown using the TPS versus static culture.
\end{abstract}

The effectiveness of the TPS in promoting osteoblastic differentiation is believed to be due to enhanced nutrient transport and exposure of the scaffold particles to shear stress. The purpose of this thesis was to quantify these two factors using computational fluid dynamics and to study the effects of altering the packing regime of the bed, the media flow rate, and the scaffold materials. The study successfully identified an average shear exposure of $0.13-0.26 \mathrm{dyn} / \mathrm{cm}^{2}$ for all cases under normal operating conditions. The highest average shear stress recorded corresponds to a chamber to bead diameter ratio (D/d) of 2.821. This case is unique because the geometry contains interior spheres. An analysis of flow within the scaffold particles determined the relative contributions of diffusive and advective transport within the scaffolds, with average Peclet numbers ranging from 0.05 to 0.30 . In more permeable tri-calcium phosphate scaffolds, advective transport plays a much more significant role. Both phenomena were found to be highly geometry dependent, with different distributions for each packing regime simulated 


\section{TABLE OF CONTENTS}

APPROVAL PAGE

ACKNOWLEDGEMENTS

ABSTRACT

TABLE OF CONTENTS V v

NOMENCLATURE vii

LIST OF TABLES viii

LIST OF FIGURES

I. INTRODUCTION 1

II. REVIEW OF RELATED LITERATURE 4

A. Tubular Perfusion Bioreactors $\quad 4$

B. Close Packing and Simulated Annealing 7

C. Shear Stress and Biosignaling 14

D. Computational Fluid Dynamics 18

E. Dimensionless Parameters 22

F. Examples of Similar Studies Using CFD 24

III. EXPERIMENTAL METHODS 27

A. Geometry Generation $\quad 27$

B. Mesh Generation $\quad 37$

C. Fluent Setup and Simulation 41

IV. RESULTS AND DISCUSSION 51

A. General Discussion and Overview

B. Shear Stress and Packing Regime $\quad 59$

C. Advective Flow and Nutrient Transport 76

D. Flow Rate Comparison $\quad 82$

E. Maximal Contact and Line Slip Structures $\quad 88$

F. Advective Transport in Tricalcium Phosphate Scaffolds 95 
V. CONCLUSION

VI. RECCOMENDATIONS

REFERENCES CITED

APPENDIX

106

VITA

109 


\section{NOMENCLATURE}

$\begin{array}{ccc}\text { As } & = & \text { Cross- Sectional Area } \\ \mathrm{D} & = & \text { Column Diameter } \\ \mathrm{d} & = & \text { Bead Diameter } \\ \mathrm{D} & = & \text { Diffusion Coefficient } \\ \mathrm{F}_{\mathrm{S}} & = & \text { Shear Force } \\ \mathrm{g} & = & \text { Gravitational Acceleration } \\ \mathrm{h} & = & \text { Enthalpy } \\ \mathrm{L} & = & \text { Peclet Number } \\ \mathrm{Pe} & = & \text { Mass Flow Rate } \\ \mathrm{Q} & = & \text { Schmidt Number } \\ \mathrm{Re} & = & \text { Sherwood Number } \\ \mathrm{Sc} & = & \text { Velocity } \\ \mathrm{Sh} & = & \text { Separation Distance } \\ \mathrm{u} & = & \text { Void Fraction } \\ \mathrm{y} & = & \text { Hydraulic Permeability } \\ \varepsilon & = & \text { Viscosity } \\ \mathrm{K} & = & \text { Density } \\ \mu & = & \text { Shear Stress } \\ \rho & = & \\ \tau & = & \\ \phi & = & \end{array}$




\section{LIST OF TABLES}

TABLE I - SUMMARY OF D/d VALUES FOR ALL PACKING REGIMES TESTED

TABLE II - CALCULATION OF VOID FRACTIONS

TABLE III - CALCULATION OF REYNOLDS NUMBERS

TABLE IV - DETERMINATION OF 20 GAUGE BEAD DIAMETERS FROM MATLAB

TABLE V - DETERMINATION OF 26 GAUGE BEAD DIAMETERS FROM MATLAB 


\section{LIST OF FIGURES}

FIGURE 1 - Crosslinking of sodium alginate with calcium ions +B3B2:B35 5

FIGURE 2 - TPS growth chamber with packed bed of alginate beads $\quad 6$

FIGURE 3 - 2D representation of simple unit cells with $\mathrm{N}$ beads in a flat plane $\quad 7$

FIGURE 4 - 3D relresentation of simple unit cells with $\mathrm{N}$ beads in a flat plane 7

FIGURE 5 - Line-slip structure (left) with corresponding maximal contact structure (right) 11

FIGURE 6 - Side view of columnar crystal with interior spheres (left); Top view of columnar crystals with and without interior spheres (right) 12

FIGURE 7 - Example image of bead before (left) and after (right) image processing 13

FIGURE 8 - Semicircular sketch used to generate spherical bead 28

FIGURE 9 - Revolve feature dialog box (left) and finished bead with sketch visible (right) 29

FIGURE 10 - Extruded Boss/Base dialog box (left) and extruded chamber (right) 30

FIGURE 11 - Shell feature dialog box (left), Face selection for shell opening (top right), Completed shell (bottom right) 31

FIGURE 12 - Four beads ready for annealing (left) and after the annealing process (right) 32

FIGURE 13 - Motion simulation panel with relevant option boxed in red 34

FIGURE 14 - Gravity dialog box (left) and contact dialog box (right) 34

FIGURE 15 - Plane creation dialog box (left) and reference planes (right) 36

FIGURE 16 - Mass properties data (left) and trimmed column for volume fraction determination (right) 36

FIGURE 17 - Named selection creation dialog for inlet surface 37

FIGURE 18 - Meshing dialog box with relevant fields highlighted in red 39

FIGURE 19 - Mesh cross-section with beads unsuppressed (left) and suppressed (right) 40

FIGURE 20 - General settings with default values 41

FIGURE 21 - Scale mesh dialog box

FIGURE 22 - Create/Edit Materials dialog box with correct constants 43

FIGURE 23 - Cell Zone Conditions dialog box with porous zone parameters defined 44

FIGURE 24 - Cell Zone Conditions panel with Physical Velocity Formulation selected 45

FIGURE 25 - Inlet boundary conditions dialog box 46

FIGURE 26 - Surface Monitor dialog box with relevant fields highlighted 48

FIGURE 27 - Run Calculation panel with number of iterations set to $100 \quad 49$

FIGURE 28 - Convergence history of scaled residuals $\quad 50$

FIGURE 29 - Convergence history of surface shear stress $\quad 50$

FIGURE 30 - Observed volume fraction for C2 - C5 with Mughal predicted values 54

FIGURE 31 - C2, C3, C4, and C5 isometric views

FIGURE 32 - Observed volume fraction for MC1-MC7 with Mughal predicted values $\quad 55$

FIGURE 33 - MC1 and MC2 (grey) compared with Mughal models generated by Monte Carlo 
FIGURE 34 - MC3 and MC4 (grey) compared with Mughal models generated by Monte Carlo

FIGURE 35 - MC5 and MC6 (grey) compared with Mughal models

FIGURE 36 - Observed volume fraction for LS1-LS3 with Mughal predicted values

FIGURE 37 - MC7 and LS1 (grey) compared with Mughal models

FIGURE 38 - LS2 and LS3 compared with Mughal models

FIGURE 39 - Composite shear average for all maximal contact structures 59

FIGURE 41 - Comparison of velocity field without (left) and with (right) interior spheres 62

FIGURE 42 - Contour map of shear distribution on C2 (D/d = 2.00) 63

FIGURE 43 - Histogram of shear magnitudes for C2 $(\mathrm{D} / \mathrm{d}=2.00)$

FIGURE 44 - Contour map of shear distribution on MC1 $(\mathrm{D} / \mathrm{d}=2.039)$

FIGURE 45 - Histogram of shear magnitudes for MC1 $(\mathrm{D} / \mathrm{d}=2.039)$

FIGURE 46 - Contour map of shear distribution on C3 (D/d = 2.155) 65

FIGURE 47- Histogram of shear magnitudes for C3 (D/d = 2.155) 65

FIGURE 48 - Contour map of shear distribution on MC2 $(\mathrm{D} / \mathrm{d}=2.225)$

FIGURE 49 - Histogram of shear magnitudes for MC2 $(\mathrm{D} / \mathrm{d}=2.225)$

FIGURE 50 - Contour map of shear distribution on MC3 (D/d = 2.291) 67

FIGURE 51 - Histogram of shear magnitudes for MC3 (D/d = 2.291) 67

FIGURE 52 - Contour map of shear distribution on C4 $(\mathrm{D} / \mathrm{d}=2.414)$

FIGURE 53 - Histogram of shear magnitudes for C4 (D/d = 2.414) 68

FIGURE 54 - Contour map of shear distribution on MC4 $(\mathrm{D} / \mathrm{d}=2.486)$

FIGURE 55 - Histogram of shear magnitudes for MC4 $(\mathrm{D} / \mathrm{d}=2.486)$

FIGURE 56 - Contour map of shear distribution on MC5 $(\mathrm{D} / \mathrm{d}=2.571) \quad 70$

FIGURE 57 - Histogram of shear magnitudes for MC5 (D/d = 2.571) 70

FIGURE 58 - Contour map of shear distribution on C5 (D/d = 2.701) 71

FIGURE 59 - Histogram of shear magnitudes for C5 (D/d = 2.701) 71

FIGURE 60 - Contour map of shear distribution on MC6 (D/d = 2.731) 72

FIGURE 61 - Histogram of shear magnitudes for MC6 (D/d = 2.731) 72

FIGURE 62 - Contour map of shear distribution on MC7 (D/d = 2.821) 73

FIGURE 63 - Histogram of shear magnitudes for MC7 (D/d = 2.821) 73

FIGURE 64 - Comparison of shear distributions between MC3 and MC7 packing regime 74

FIGURE 65 - \% of surface $>0.15 \mathrm{dyn} / \mathrm{cm}^{2}$ for each packing regime $\quad 75$

FIGURE 66 - Average Peclet number as a function of packing regime 77

FIGURE 66 - Average Peclet number as a function of packing regime 78

FIGURE 68 - Vector plot for C4 showing flow striking beads perpendicular to surface $\quad 79$

FIGURE 69 - Velocity Contours on representative bead surface for C4 80

FIGURE 70 - Cross section of C4 bead showing Pe >1 (left) and Pe > 0.1 (right) 80

FIGURE 71 - Velocity Contours on representative bead surface for C2 81

FIGURE 72 - Cross section of C2 bead showing Pe >1 (left) and Pe $>0.1$ (right) 81

FIGURE 73 - Average shear stress values for MC3 and MC7 at 3 and $10 \mathrm{~mL} / \mathrm{min}$ 
FIGURE 74 - Shear stress distributions for MC3 at 3 and $10 \mathrm{~mL} / \mathrm{min} \quad 83$

FIGURE 75 - Shear stress distributions for MC7 at 3 and $10 \mathrm{~mL} / \mathrm{min} \quad 84$

FIGURE 76 - Average Peclet number at 3 and $10 \mathrm{~mL} / \mathrm{min} \quad 85$

FIGURE 77 - Peclet number distributions by $\%$ of scaffold volume at 3 and $10 \mathrm{~mL} / \mathrm{min} \quad 85$

FIGURE 78 - Regions of Pe >1 in MC3 at $3 \mathrm{~mL} / \mathrm{min}$ (left) and $10 \mathrm{~mL} / \mathrm{min}$ (right) 86

FIGURE 79 - Regions of Pe $>0.1$ in MC3 at $3 \mathrm{~mL} / \mathrm{min}$ (left) and $10 \mathrm{~mL} / \mathrm{min}$ (right) 87

FIGURE 80 - Regions of Pe >1 in MC7 at $3 \mathrm{~mL} / \mathrm{min}$ (left) and $10 \mathrm{~mL} / \mathrm{min}$ (right) 87

FIGURE 81 - Regions of Pe $>0.1$ in MC7 at $3 \mathrm{~mL} / \mathrm{min}$ (left) and $10 \mathrm{~mL} / \mathrm{min}$ (right) 88

FIGURE 82 - Mean shear stress for each line slip/maximal contact pair 89

FIGURE 83 - Shear distribution for LS1 and C4 by \% of scaffold surface 90

FIGURE 84 - Shear stress contour plots for LS1 (left) and C4 (right) 91

FIGURE 85 - Top-down shear stress contour plots for LS1 (left) and C4 (right) 91

FIGURE 86 - Shear stress distribution for LS2 and MC5 by \% of surface area 92

FIGURE 87 - Shear stress contour plots for LS2 (left) and MC5 (right) 92

FIGURE 88 - Top down shear stress contour plots for LS2 (left) and MC5 (right) 93

FIGURE 89 - Shear stress distribution for LS3 and C5 by \% of surface area 93

FIGURE 90 - Shear stress contour plots for LS3 (left) and C5 (right) 94

FIGURE 91 - Top down shear stress contour plots for LS3 (left) and C5 (right) 94

FIGURE 92 - Comparison of average Peclet number for both packing regimes and materials 96

FIGURE 93 - \% of scaffold volume at Pe $>1$

FIGURE 94 - Regions of Pe >1 on MC3 in alginate (left) and tricalcium phosphate (right) 97

FIGURE 95 - Regions of Pe > 1 on MC7 in alginate (left) and tricalcium phosphate (right) 97 


\section{INTRODUCTION}

The engineering of three dimensional tissues is a new frontier in biomedical engineering, with a promising array of future clinical applications. Though scientists have been able to culture populations of cells in vitro for many decades, the science of growing three-dimensional constructs for implantation into the body is still emerging, and comes with a unique set of challenges. Research labs around the globe have had much success in the field of tissue engineering in a lab setting, but the continued development of novel bioreactors and new biomaterials to serve as scaffolds is necessary to advance tissue engineering to the clinical level. Though there is still much work to be done, the impetus for such advancements is strong. As technology improves, bioreactors for tissue engineering become ever more versatile tools for a variety of serious ailments, including the culturing of new islet cells to restore insulin production in type I diabetics, the development of bone grafts that heal more quickly and with less complications, and the production of new ligaments for athletes with ACL injuries, with many more applications sure to come.

Growing tissues in a bioreactor, regardless of the application, always begins with traditional cell culture, using a population of stem cells appropriate for the application. In a clinical setting, these cells would be taken from the patient who is to receive the tissue, eliminating the possibility of an immunogenic response, a significant complication in tissue and organ transplantation. Once the stem cell population has been prepared, these cells are encapsulated in a porous material that serves as a scaffold for the nascent cells to grow and produce extracellular matrix. The cell-seeded scaffolds are then placed into a bioreactor designed to promote the proliferation of the cells throughout the scaffold material, and to promote their differentiation into 
the target cell type. Unlike in traditional culture where cells are grown along the bottom of a petri dish or flask, in constant contact with the growth media, cells encapsulated inside a scaffold material are by nature separated from the growth media, and must rely on mass transport through the scaffold to carry nutrients and growth factors in and waste products out. In most cases, when the scaffolds are placed in static media, simple diffusion alone is insufficient for supplying the cells with oxygen and nutrients, and cell death occurs rapidly (Fischer 2011). This underscores the first key challenge in designing a bioreactor system for 3D tissue culture: the bioreactor must provide sufficient mass transport of nutrients to keep the nascent cells alive until the tissue is ready for transplantation, a matter of days or weeks. In order to accomplish this, all bioreactors use some sort of flow field to exploit convective mass transport. This challenge becomes even more pressing as bioreactor systems are scaled up from benchtop scale apparatus with millimeter scale scaffolds, to more clinically relevant sizes.

Simply keeping the cells alive inside of a bioreactor is not enough to ensure success when growing tissues in vitro. The environment in the bioreactor must also promote the differentiation of stem cells into the target cell type. This is accomplished by a combination of both chemical and mechanical cues. This paper is particularly focused on a bioreactor design for bone tissue engineering, where mechanical stimulation is a key mediator of the biosignaling pathways that lead to osteoblastic differentiation. Specifically, research shows a strong link between shear exposure and the expression of bone extracellular matrix proteins. These mechanically dependent pathways likely developed through osteoblasts' natural environment in the body, where constant cyclic loading through walking and activity produces shear forces. However, shear forces above a certain magnitude are detrimental to cell survival. An ideal bioreactor for bone tissue applications 
would ensure uniform shear stress over the scaffold surface, of the appropriate magnitude to promote differentiation without harming the cells.

With the two key challenges of mass transport and shear exposure in mind, it is desirable to have a way to determine how well a bioreactor performs in these two categories. Experimental determination of shear exposure within a bioreactor with a complex geometry is in many cases impractical, thus computational fluid dynamics is an attractive option that has been employed in a number of studies. Benchtop studies with bioreactor systems take weeks to complete, and rely on indirect metrics to assess efficacy, such as histological analysis and quantitative PCR to determine levels of protein expression. While these data are useful, CFD allows for the direct study of mass transport and shear exposure under a variety of conditions with relative ease, allowing researchers to quantify the effects of scaffold size, scaffold permeability, and media flow rate.

The focus of this thesis is the development of a CFD model for a novel bioreactor known as a tubular perfusion bioreactor, developed for bone tissue applications by Dr. John Fisher at the University of Maryland. A simplified 2-D model was developed previously using COMSOL, but previously no attempt has been made to generate a full 3-D model. This model will be used to study the magnitude and distribution of shear stress on the surface of the scaffold beads, the contribution of advective flow within the pores of the material to overall nutrient transport, and how the size and packing regime of the scaffold beads affects these key parameters. These data can be directly used to improve the design of the bioreactor, and the model provides a powerful proof of concept for the use of CFD in bioreactor optimization. 


\section{LITERATURE REVIEW}

\section{A. Tubular Perfusion Bioreactors}

A common solution to the problem of growing tissues in three dimensions is the use of perfused bioreactor systems, through which growth media is constantly perfused by way of a peristaltic pump. Designs vary widely, but in general a perfused bioreactor consists of a closed circuit of tubing with a media reservoir, a pump, and a growth chamber where the scaffold resides.

The key difference between different perfusion bioreactors is in the design and fabrication of this growth chamber. In bone tissue engineering, rigid ceramic and composite materials such as tri-calcium phosphate or PLGA often serve as scaffolds due to their mechanical strength. Because these materials are highly permeable, bioreactors for this application often consist of a flat chamber with a solid plug of scaffold material through which all media must pass, such as those described by Patrarchi et. al. (2012). This design is impractical for less permeable materials with smaller intrinsic pore sizes, such as those used in soft tissue and cartilage engineering, as a great deal of pressure would be required to force the media through the relatively impermeable scaffold plug. This design could cause the bioreactor to leak and could subject the cells to very high shear. In these applications, templated scaffolds are used with specially designed channels, such as the one described by De Boodt et. al. (2010). The size and geometry of these channels has a profound effect on the shear and mass transport within, and previous work using computational fluid dynamics has been mainly focused on the spatial optimization of channels in this type of bioreactor.

Unlike the designs described in the preceding paragraph, the tubular perfusion system (TPS) designed by Fisher et. al. utilizes a packed bed of spherical scaffold beads inside a cylindrical length of silicone tubing. According to Fisher, this design carries many advantages over 
other perfusion systems, as it requires no special fabrication techniques, provides a large surface area exposed to shear, and works with a variety of materials since the bulk of the flow occurs in the void space, where the pore size and interconnectivity of the scaffold material are not a concern. Though this design is novel in the field of bone tissue engineering, tubular growth chambers are commonly used in engineering blood vessels, where vascular tissue is grown along the inner wall of the chamber (Fisher 2011). Packed bed reactors also have numerous uses in biomedical applications, including the generation of recombinant proteins.

In Fisher's studies, the scaffold beads used in the TPS are made with alginate, a very hydrophilic anionic polysaccharide. A $2 \% \mathrm{w} / \mathrm{v}$ solution of powdered sodium alginate in $0.15 \mathrm{M}$ $\mathrm{NaCl}$ is first created. The stem cells to be added are then separated from their culture media using trypsin/EDTA and pelleted via centrifugation. This pellet of cells is dissolved in the alginate solution, which is then added dropwise to a solution of $0.1 \mathrm{M} \mathrm{CaCl}$ using a syringe. Exposure to the calcium ions in solution induces the polysaccharide chains to crosslink and creates solid spherical beads. The crosslinking reaction is demonstrated in FIGURE 1.

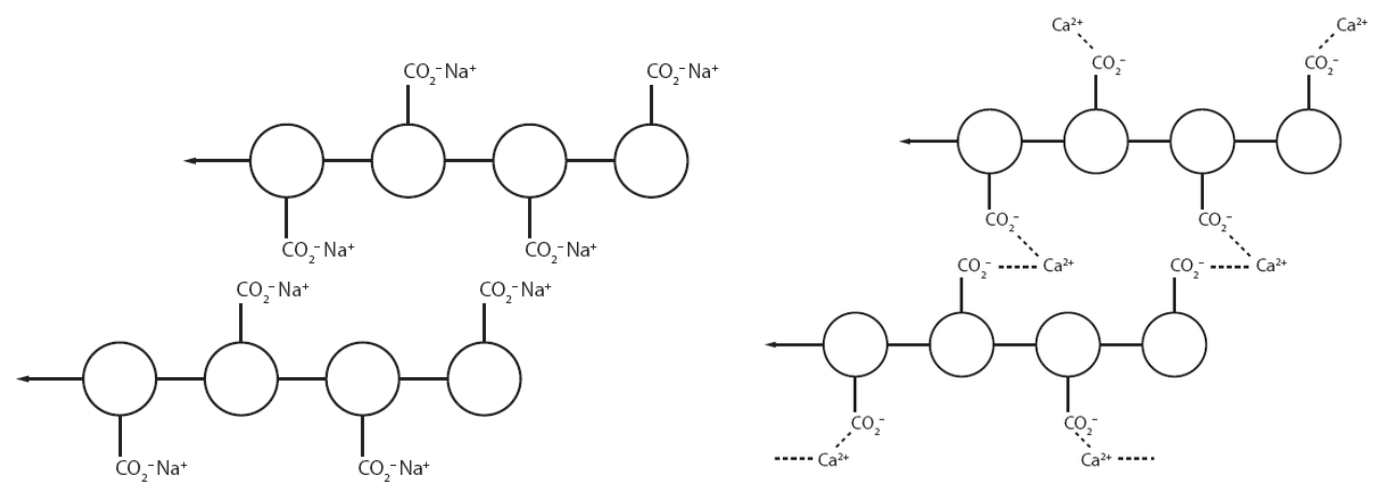

FIGURE 1 - Crosslinking of sodium alginate with calcium ions 
These beads are allowed to stabilize for 15 minutes to ensure that the crosslinking process goes to completion. Once ready, the beads are added to the growth chamber and gravity allows them to settle into place. The density and permeability of the beads can be tuned by altering the concentration of the alginate solution. By using different diameter needles to add the alginate dropwise to the crosslinking solution, the size of the beads can likewise be altered. According to Fisher, alginate is a good candidate for bone tissue engineering because it can be easily dissolved using a calcium chelating agent after the tissue has developed, leaving behind only cells and extracellular matrix. This is different than the traditional approach of using more rigid scaffold materials that are designed to degrade after implantation into the body. It also gives the system versatility, as the TPS can also be used for soft tissue engineering. FIGURE 2 shows a representative growth chamber filled with a packed bed of alginate beads, artificially dyed for visibility.

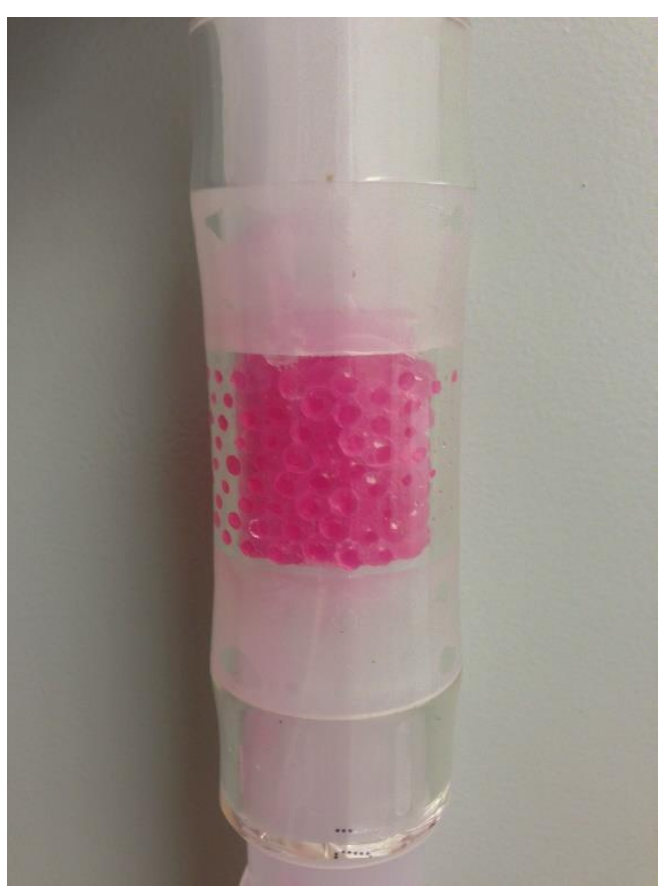

FIGURE 2 - TPS growth chamber with packed bed of alginate beads 


\section{B. Close Packing and Simulated Annealing}

In perfusion bioreactors with scaffolds created by templating or additive manufacturing, flow characteristics such as shear stress can be altered by altering the geometry of the flow channels. Studies such as the one conducted by He et. al (2011) and Papantoniou et. al. (2014) utilize computational fluid dynamics to optimize the geometry of such systems. Due to the nature of the tubular perfusion system, the channels for fluid flow are not pre-designed, but are dependent on the packing structure of the scaffold beads inside the growth chamber. The void fraction of the packed column and the tortuosity of the void space through which fluid will flow have a profound effect on the flow pattern in the column. These variables can be controlled by altering the size of the scaffold beads. Quantifying the effects of scaffold size and packing regime on shear and mass transport is the key goal of this thesis.

The packing structure of spherical particles inside a spherical container is a function of the ratio of the container diameter to the bead diameter, D/d. According to Mughal and Chan (2012), each discrete diameter ratio naturally forms a unique columnar crystal, a structure that can be formed by repeatedly stacking unit cells along the axial length of the column. The number of particles in a single unit cell and the shape of the unit cell is different for each value of $D / d$, but can be determined either through simple deduction or mathematical simulation. The simplest cases involve unit cells which lie entirely in a flat plane perpendicular to the axis of the cylindrical chamber. Finding the structure of these cases is analogous to the $2 \mathrm{D}$ problem of finding the smallest diameter circle into which $\mathrm{N}$ non-overlapping smaller circles can be packed. Such structures are illustrated in FIGURE 3 and FIGURE 4. 

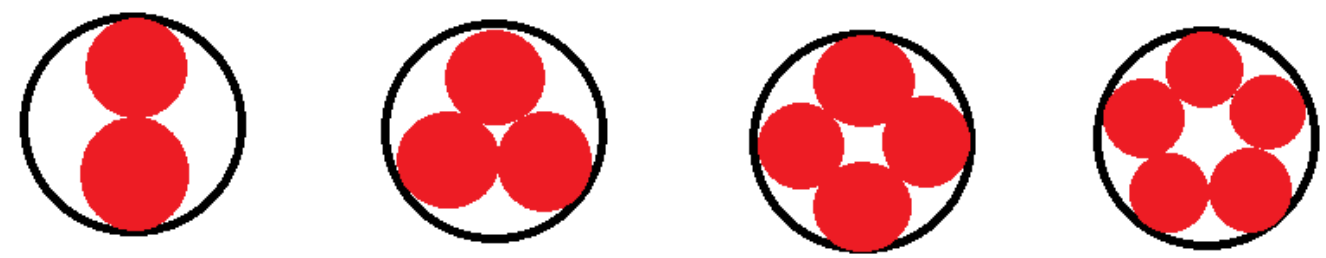

FIGURE 3-2D representation of simple unit cells with $\mathrm{N}$ beads in a flat plane
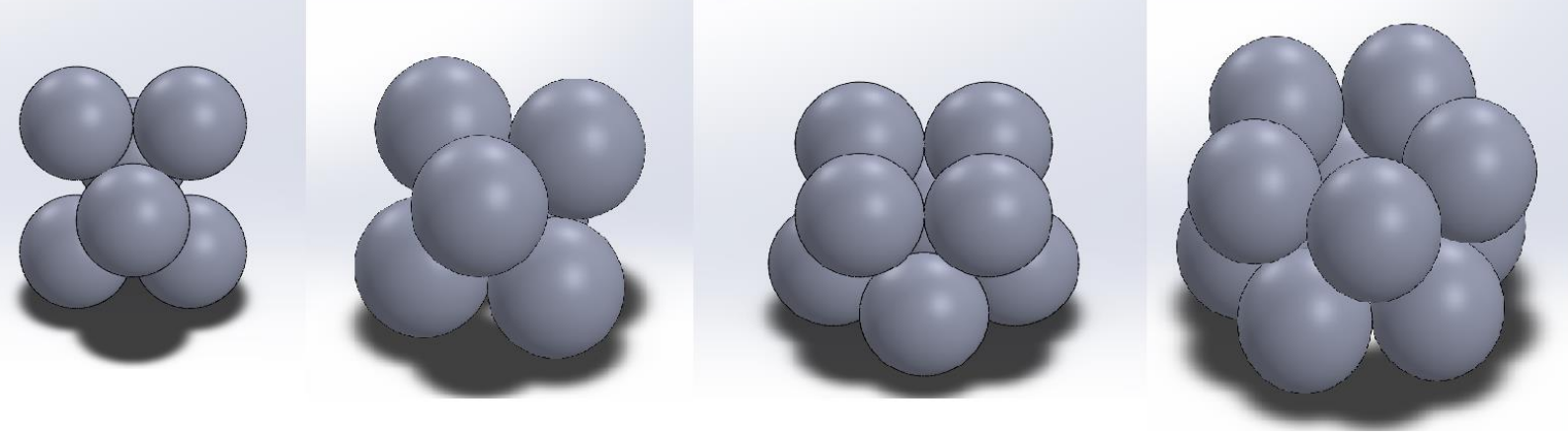

FIGURE 4 - 3D representation of simple unit cells with $\mathrm{N}$ beads in a flat plane

For most D/d values, the corresponding unit cell structure is not easily deduced. In these cases, mathematical modeling is used to find the columnar crystal structure in which void fraction is minimized, a process known as simulated annealing. A computational algorithm is used to find a representative unit cell, consisting of $\mathrm{N}$ spheres, which can be extrapolated into a columnar crystal by translation along the cylinder axis by a distance $\mathrm{nL}$, and rotation about the cylinder axis 
by angle na (Mughal and Chan, 2012). This is known as a "screw operation" and is easily visualized in the simple structures pictured in FIGURE 4. For instance, in the first example containing 2 beads per unit cell, the twist angle $\alpha$ would be equal to $90^{\circ}$.

The simulated annealing algorithm used by Mughal and Chan begins by defining two potential functions, one for interaction between the spheres, $E_{i j}^{S}$, and one for interaction between the spheres and boundary, $E_{i}^{B}$. The potential functions are modeled after spring behavior, according to Hooke's Law. If two spheres are placed close enough to overlap, $E_{i j}^{S}$ will be nonzero. Likewise, if a sphere penetrates the outer boundary of the cylinder, $E_{i}^{B}$ will be nonzero.

$$
\begin{gathered}
E_{i j}^{S}= \begin{cases}\frac{1}{2}\left(r_{i j}-d\right)^{2}, & r_{i j}<d \\
0, & r_{i j} \geq d\end{cases} \\
E_{i B}= \begin{cases}\frac{1}{2}\left(r_{i B}-d / 2\right)^{2}, & r_{i j}<\frac{d}{2} \\
0, & r_{i j} \geq \frac{d}{2}\end{cases}
\end{gathered}
$$

These two equations function as convergence criteria. The Metropolis-Hastings algorithm, a Monte Carlo method, is then used to determine the spatial configuration at which potential is minimized for a given unit cell length. The unit cell length is gradually increased until the potential functions are reduced to zero. This point represents the densest spatial configuration for a given D/d ratio with no overlapping of the spheres. In Mughal's studies, a robust convergence criteria was applied, simulating $\mathrm{N} \times\left(5 \times 10^{6}\right)$ Monte Carlo steps for each run, then repeating each run multiple times with a different randomly generated starting configuration, to ensure the same optimal configuration is reached each time. Additionally, for a given $\mathrm{D} / \mathrm{d}$, the entire procedure is repeated for different numbers of spheres, $\mathrm{N}$, from 1 to 15 . The result which yields the smallest void fraction is considered the final result. 
Mughal and Chan have applied this method to all D/d ratios $\leq 2.873$ with some counterintuitive results. One might assume that as the value of $\mathrm{D} / \mathrm{d}$ is increased, void fraction decreases linearly as smaller spheres are able to pack more tightly. However, the results from simulated annealing show that void fraction oscillates as $\mathrm{D} / \mathrm{d}$ is increased, traversing several maxima and minima. The minima correspond to points at which a maximum number of contact points has been reached. If the diameter of the spheres were made any larger, they would begin to overlap, making the given solution overconstrained. These points mark the transition from one packing regime to the next. At these points, the derivative of the void fraction with respect to D/d approaches $-\infty$. These special solutions are called "maximal contact structures." The simple unit cells shown in FIGURE 4 are a subset of this category. Taken together, the maximal contact structures define all possible packing regimes within the given range of diameter ratios. As such, each maximal contact structure characterized by Mughal was simulated as a part of this study. The intervening crystal structures between the maximal contact structures are modified versions of the maximal contact structures, in which void fraction reaches a minimum. These structures are best characterized as "staggered helices" in which the contact pattern within the unit cell, and thus the packing regime, remains the same. In these cases, however, the contact points between the spheres have slipped enough to accommodate the new sphere diameter. Mughal calls these structures "lineslip" structures. A comparison between a maximal contact structure and the corresponding lineslip structure is shown in FIGURE 5. 

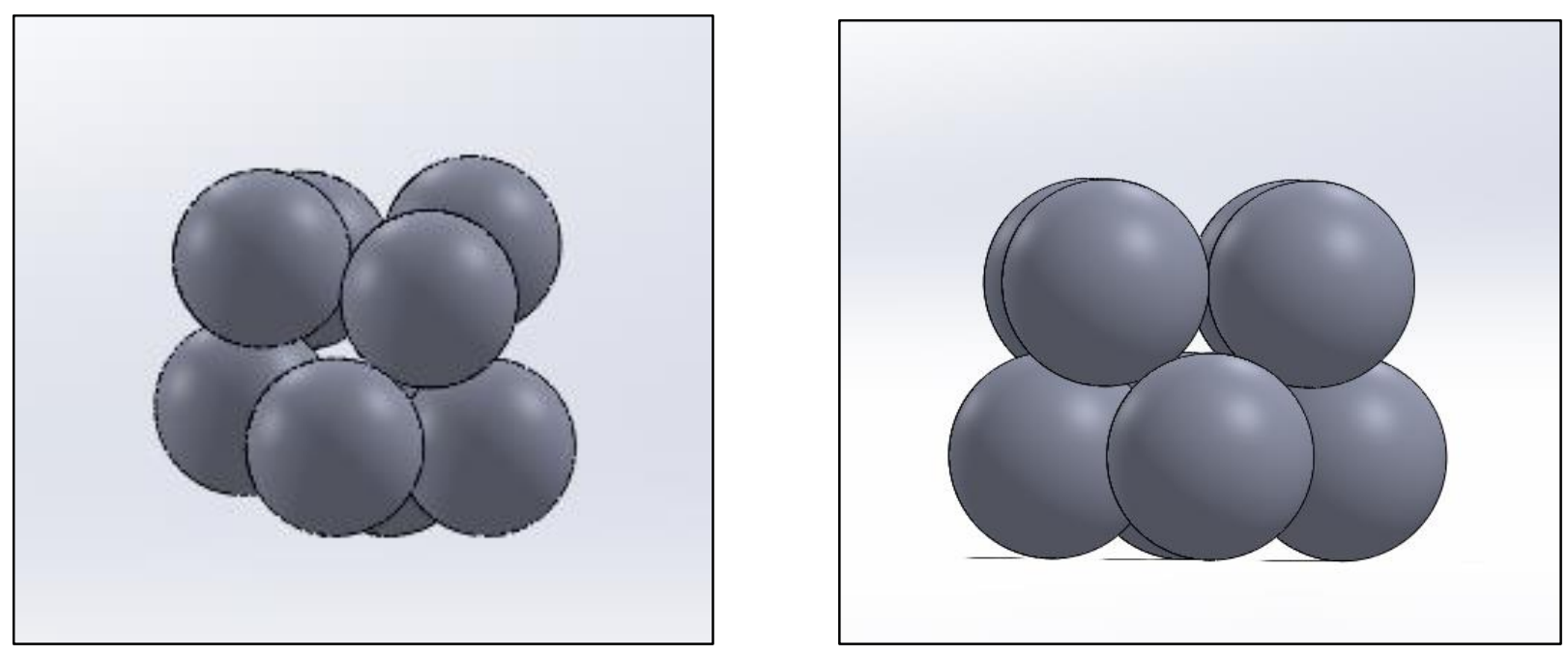

FIGURE 5 - Line-slip structure (left) with corresponding maximal contact structure (right)

Differences in void fraction over all of the structures tested are slight, ranging from about 46-48\%. It is therefore hypothesized that shear coverage and advective flow within the scaffold particles is likely more a function of the packing structure and tortuosity of the fluid pathways than the numerical value of the void fraction. One crucial factor affecting the tortuosity of the fluid pathway is the presence of interior spheres. For all structures with $\mathrm{D} / \mathrm{d} \leq 2.71$, the optimal packing regime is such that all spheres contact the cylinder wall. This leaves a hollow core through the center of the cylinder where no beads reside. For $\mathrm{D} / \mathrm{d}>2.71$, interior spheres begin to appear. This leads to the second hypothesis addressed by this thesis: shear stress and mass transport can be maximized by selecting $\mathrm{D} / \mathrm{d}$ ratios which contain interior spheres. It stands to reason that this would be true, since in the case where there are no interior spheres, fluid flow will be directed mostly through the hollow central core, exposing little of the surface of the spheres to shear. FIGURE 6 illustrates packing regimes with and without interior spheres. 

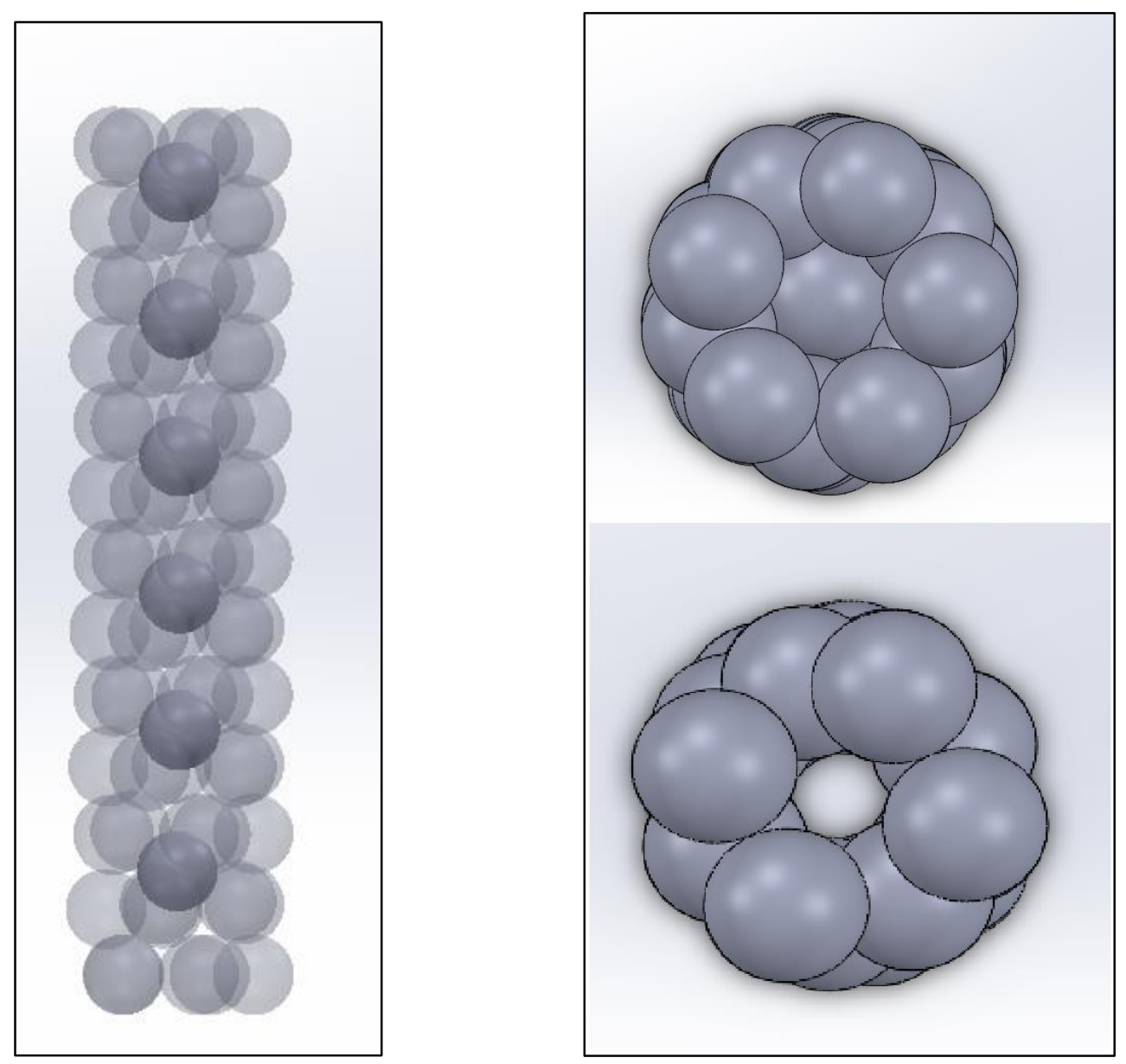

FIGURE 6 - Side view of columnar crystal with interior spheres (left); Top view of columnar crystals with and without interior spheres (right)

In a laboratory setting, the annealing of scaffold beads into a columnar crystal occurs by careful addition of the beads to the growth chamber, few at a time, followed by tamping the column down after each addition. If care is taken to spot stacking faults as they occur, the ideal packing structures generated by simulation can be duplicated in the laboratory. It stands to reason that the packing structure of the scaffold beads will have a profound effect on the efficacy of the bioreactor. As such, choosing an optimal diameter for the spheres according to simulation data is the chief goal of this study. To date, scaffold beads have been generated using 20 gauge and 26 gauge needles. In preparation for this study, a population of beads created using each needle gauge was 
imaged under magnification and sized using a MATLAB script. This image processing identifies the region of the image occupied by the bead, locates the centroid of the bead and determines an average diameter. These trials found an average diameter of 2.75 and $2.23 \mathrm{~mm}$, respectively, corresponding to a $\mathrm{D} / \mathrm{d}$ ratio of 2.309 and 2.848 . In this range, the packing regime will match that of maximal contact structures MC3 and MC7, as denoted in the results section. The raw data on bead size is reported in the appendix of this report.
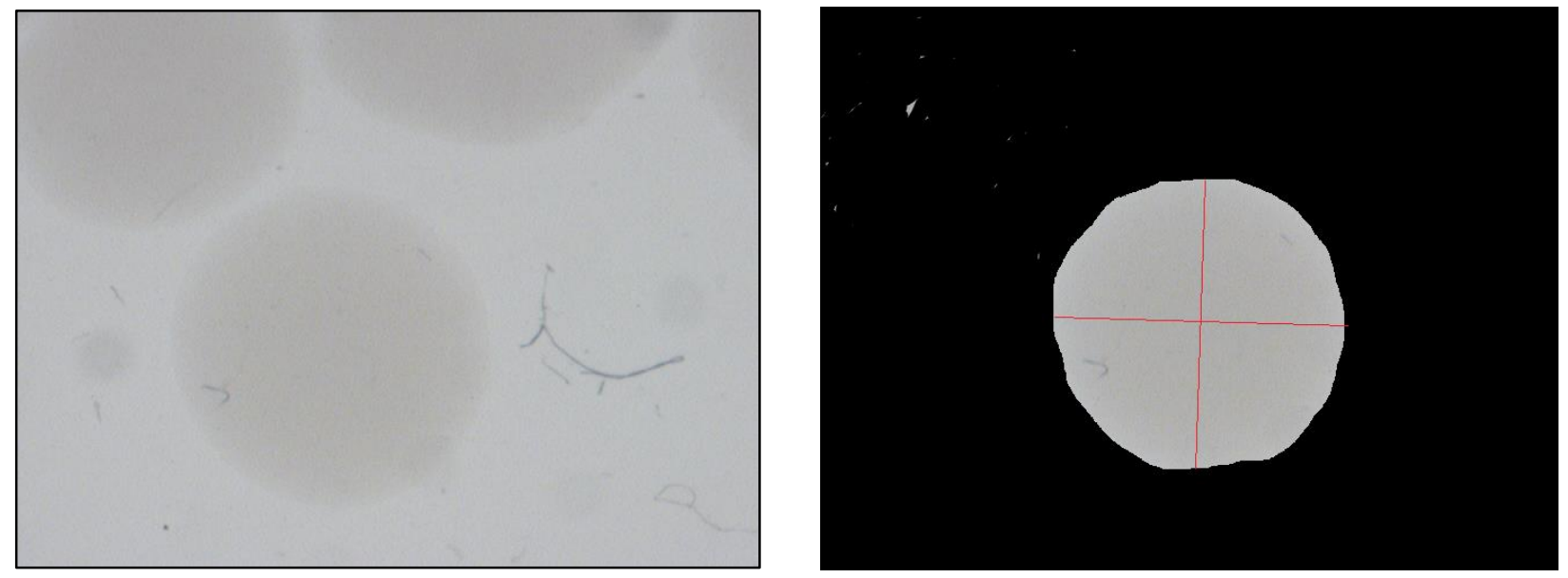

FIGURE 7 - Example image of bead before (left) and after (right) image processing 


\section{Shear Stress and Biosignaling}

While the focus of this thesis is primarily modeling, some background is needed on the meaning of shear and its role in the biosignaling pathways leading to osteoblastic differentiation. Shear stress is defined by Bird et. al. (2002) using a system consisting of two parallel plates, one moving and one stationary, separated by a liquid. Due to friction between the liquid molecules, a force is required to maintain the steady motion of the plate. At both boundaries, the velocity of the fluid relative to the boundary is taken to be zero, known as the no-slip boundary condition. Thus, a velocity gradient develops between the two plates. The force that must be applied to maintain this velocity gradient is directly proportional to the area of the plate and the velocity at which it is moving and inversely proportional to the separation distance. The proportionality constant, $\mu$, represents the viscosity of the liquid, a material property which represents the resistance to momentum transport through the fluid.

$$
\frac{F_{s}}{A_{s}}=-\mu \frac{u}{y}
$$

For systems with more complex boundaries, a linear velocity gradient will not always be present. Newton's Law of Viscosity provides a more general definition of shear stress, related to the instantaneous velocity gradient at the surface:

$$
\tau_{x y}=-\mu \frac{d u_{x}}{d y}
$$

In this form of the equation, $\tau_{x y}$ represents the shear stress, or the force in the $\mathrm{x}$ direction per unit area perpendicular to the y direction. This equation applies for one-dimensional laminar flow of Newtonian fluids. 
The importance of shear stress in bone tissue engineering is a well-studied phenomenon. According to Sikavitsas et. al. (2001), the cyclic loading and unloading of bone tissue in vivo generates flow of interstitial fluid inside the pores of the bone. This fluid flow sets in motion a complex mechanotransduction system that regulates the production of numerous growth factors and influences the differentiation of osteoprogenitor cells into their target cell type. In the TPS, the progenitor cells used are human mesenchymal stem cells (hMSCs), a type of stem cell found in bone marrow which can differentiated into osteoblasts (bone cells), chondrocytes (cartilage cells), myocytes (muscle cells) or adipocytes (fat cells). Research has shown that their ultimate fate depends entirely on the conditions they are subjected to in the bioreactor, and that shear exposure is necessary for differentiation into osteoblasts.

Yeatts and Fisher (2012) explore the biochemical pathways influenced by shear. Mechanical stresses activate a signaling cascade mediated by mitogen activated protein kinases (MAPKs). These kinases in turn activate three key signaling pathways, the JNK, p38, and ERK 1/2 pathways. The downstream targets of each pathway are transcriptional regulators, which increase production of a variety of enzymes and growth factors. Two key downstream targets whose levels are often monitored in bone tissue engineering studies are alkaline phosphatase (ALP) and osteopontin (OPN). Alkaline phosphatase is a versatile enzyme used to dephosphorylate a variety of biomolecules, while osteopontin regulates mineralization. The expression of these two enzymes is a good indicator of osteoblastic differentiation.

Numerous studies have been conducted on the exact magnitude of shear stress necessary for enhanced osteoblastic differentiation in vitro. According to Sikavitsas (2001), bone cells in vivo are routinely exposed to shear stresses of $8-30 \mathrm{dyn} / \mathrm{cm}^{2}$. However, studies suggest that shear stresses begins to influence signaling pathways at a much lower magnitude. A study by Bancroft 
et. al. (2002) showed greatly enhanced OPN expression and calcium matrix deposition with shear stress exposure less than $1 \mathrm{dyn} / \mathrm{cm}^{2}$. Mature osteoblasts produce the calcium matrix, thus the presence of extensive matrix deposition indicates that the majority of the cells have differentiated to osteoblasts. Likewise, Kreke et. al.(2005) demonstrated an increase in OPN expression in a population of stromal cells placed in a parallel plate reactor and subjected to shear of $1.6 \mathrm{dyn} / \mathrm{cm}^{2}$ for differing lengths of time.

While perfusion systems clearly promote enhanced osteoblastic differentiation, it is possible that the results presented in the preceding paragraph are also due in part to enhanced nutrient transport, since increased shear typically means an increase in flow rate and a corresponding increase in mass transport. Li et. al. (2009) performed a study in which the effects of flow rate and mass transport were evaluated separately. This was accomplished by raising the viscosity of the growth media with the addition of dextran, thus increasing shear without altering the flow rate. This study showed that increasing shear from 0.05 to $0.15 \mathrm{dyn} / \mathrm{cm}^{2}$ without altering the media flow rate increased mineralization and OPN expression. This illustrates that shear alone is influential in cell proliferation and differentiation, and also the low threshold required. Li also studied the effects of mass transport while holding shear stress constant, varying the flow rate from $0-9 \mathrm{~mL} / \mathrm{min}$. Again, osteopontin activity increased with increasing flow rate, but an inhibitory effect was observed at flow rates above $6 \mathrm{~mL} / \mathrm{min}$. Once adequate nutrient transport has been achieved, higher flow rates seem to interfere with cell signaling and matrix deposition.

Though the effects of direct shear exposure have been well characterized, the question remains whether shear exposure can have a profound effect in the 3D culture environment of the TPS. Since the beads are tightly porous and the majority of the media flow will be directed around the outside of the beads, shear exposure will be much less for the cells seeded on the interior of the 
scaffold beads due to the very low advective flow rate beyond the surface. In another study, Yeatts and Fisher (2012) investigated the connection between the position of cells within the scaffold and their ultimate fate. In this study, histomorphometric analysis was used to quantify proliferation and mineralization at different radial points within the scaffold beads. Though less mineralization was observed in the inner annulus than the outer annulus of the scaffold beads after 28 days in the bioreactor, both showed a significant increase over static culture. Additionally, an increase in osteoblastic differentiation across the entire cross section of the bead was observed when the surface shear was increased by the addition of dextran to the media. Aside from the benefits of enhanced mass transport, Yeatts and Fisher postulate that paracrine signaling from the cells along the surface of the beads in response to shear is responsible for signaling the interior cells to differentiate.

Thus far, the only quantification of the magnitude of surface shear produced in the TPS comes from a simplified 2D model generated by the Fisher group using COMSOL multiphysics. In the study described in the preceding paragraph, dextran was again used as a thickening agent, to study the effects of different shear magnitudes on osteoblastic differentiation. Two different viscosities were tested, corresponding to two dextran concentrations. All studies were conducted with a media flow rate of $3 \mathrm{~mL} / \mathrm{min}$. An average shear stress of $1.63 \pm 0.13 \mathrm{dyn} / \mathrm{cm}^{2}$ was calculated for a $3 \%$ dextran solution $(\mu=1.66 \pm .05 \mathrm{mPa} \mathrm{S})$, and $4.13 \pm 0.34 \mathrm{dyn} / \mathrm{cm}^{2}$ was calculated for a $9 \%$ dextran solution. Likewise, the permeability of the scaffold was modeled using COMSOL and an average advective flow rate within the media was found to be $3 \times 10^{-7} \mathrm{~mL} / \mathrm{min}$. This two dimensional model is severely limited in its capabilities. In two dimensions, it is impossible to replicate the wide variety of packing regimes possible within the growth chamber of the TPS. Additionally, shear coverage over the entire surface of a bead can't be investigated, since the spherical beads are 
simplified to circles. The motivation for developing the $3 \mathrm{D}$ model outlined in this thesis is to overcome these shortcomings and to develop a powerful tool for measuring surface shear and nutrient transport.

\section{Computational Fluid Dynamics}

The Navier-Stokes Equation, Fick's Law, and Fourier's Law together comprise the canonical equations for momentum, mass, and heat transport, respectively. With an understanding of these equations, a variety of simple problems related to transport phenomena can be solved with ease. Many engineering problems, however, are far too complex to be solved by hand. At each discrete point in a complex flow field, the fluid velocity, density, mass flux or heat flux might vary in a complex engineering problem. Computers, however, can perform iterative solutions many orders of magnitude faster than humans. Computational fluid dynamics software allows complex flow fields to be discretized into hundreds of thousands or even millions of elements. This is accomplished by generating a computational mesh, a grid which represents a solid geometry. The software then iteratively solves all of the relevant transport equations at each element until a solution is reached. This technology is widely used in a wide variety of fields, including aerodynamics, HVAC, and reactor design, among many others.

There exist a number of commercial packages for computational fluid dynamics, including Ansys Fluent, CFX, and OpenFOAM. For this study, all simuluations were conducted in Ansys Fluent. Most CFD packages, however, operate on similar principles. As such, this study could be replicated using a different software package. 
In Ansys Fluent, the governing equations relevant to the bioreactor simulation according to the Fluent Theory Guide are as follows:

For conservation of mass:

$$
\frac{\partial \rho}{\partial t}+\frac{\partial}{\partial x_{i}}\left(\rho * u_{i}\right)=S_{m}
$$

For conservation of momentum:

$$
\frac{\partial}{\partial x_{i}}\left(\rho * u_{i}\right)+\frac{\partial}{\partial x_{j}}\left(\rho * u_{i} * u_{j}\right)=-\frac{\partial \rho}{\partial x_{i}}+\frac{\partial \tau_{i j}}{\partial x_{j}}+\rho * g_{i}+F_{i}
$$

For conservation of energy:

$$
\frac{\partial}{\partial t}(\rho * h)+\frac{\partial}{\partial x_{i}}\left(\rho * u_{i} * h\right)=\frac{\partial p}{\partial x_{i}}\left(k * \frac{\partial T}{\partial x_{i}}\right)-\frac{\partial}{\partial x_{i}} \sum h_{j} * J_{j}+\frac{\partial p}{\partial t}+u_{i} \frac{\partial p}{\partial x_{i}}+\tau_{i j} \frac{\partial u_{i}}{\partial x_{j}}+S_{h}
$$

The variables in the preceding equation are defined as:

$$
\begin{gathered}
\mathrm{h}=\sum m_{i} * h_{i} \\
h_{i}=\int_{T r e f}^{T} C_{p i} d T
\end{gathered}
$$

For calculation of shear stress:

$$
\tau_{i j}=\left[\mu\left(\frac{\partial \tau_{i}}{\partial x_{j}}+\frac{\partial \tau_{j}}{\partial x_{i}}\right)\right]-\frac{2}{3} * \mu * \frac{\partial \tau_{i}}{\partial x_{i}} * \delta_{i j}
$$


For conservation of chemical species:

$$
\frac{\partial}{\partial t}\left(\rho * m_{i}\right)+\frac{\partial}{\partial x_{i}}\left(\rho * u_{i} * m_{i}\right)=\frac{\partial}{\partial x_{i}}\left(J_{i^{1} i^{2}}\right)+S_{i}
$$

Where $\mathrm{J}$ is defined as:

$$
J_{i, i^{2}}=-\rho * D_{i^{1,}, i^{2}} * m * \frac{\partial m_{i}}{\partial x_{i}}
$$

To set the stage for an iterative solution, fluent uses the finite difference method. This is a numerical method for solving complex differential equations by converting them to difference equations. Like the Euler Method, the finite differences method approximates the derivative at a point and uses this derivate to find the next iterative solution.

The discretization method used by fluent is known as the finite volume method. Once the computational mesh has been generated by dividing the geometry into tetrahedrons or hexahedrons, each element is treated independently by the software. The momentum balance equation is solved in each element independently to determine the $\mathrm{x}, \mathrm{y}$, and $\mathrm{z}$ momentum components. The solution for the velocity field is iterated until the continuity equation is satisfied for each element, that is to say that flow into the element equals flow out of the element. To monitor for convergence, fluent records "residuals" after each iteration, which represent the magnitude of change from the previous iteration. If the problem converges correctly, the residuals should decrease rapidly. The threshold at which the solution is considered converged is set by the user.

Special auxiliary equations are needed in dealing with porous or permeable media, such as in the TPS model. The necessary inputs are based on Darcy's Law. Darcy's Law relates flow rate of a liquid through a permeable media to the pressure gradient applied to the media, the surface area and thickness of the permeable media, and the viscosity of the liquid. Darcy permeability, 
denoted $\mathrm{k}$, is an intrinsic material property that does not change with changes in geometry.

Darcy's Law:

$$
Q=-\frac{\kappa A}{\mu} \frac{\left(P_{B}-P_{A}\right)}{L}
$$

Fluent has two schemes for solving velocity fields in porous media: the superficial velocity formulation, or the physical velocity formulation. The superficial velocity formulation is more computationally simple, as it assumes a homogeneous velocity throughout the porous media based on bulk flow rate through the region. This formulation is suitable for applications where the chief concern is pressure drop through a porous region. However, since this study is largely concerned with the flow fields inside the scaffold beads, the physical velocity formulation must be used. This solves for the true velocity through the porous media by treating the porous media as an isentropic momentum sink. This adds an extra term to the momentum balance equation, accounting for viscous and inertial losses within the porous zone.

Momentum source term for porous zones:

$$
S_{i}=-\left(\sum_{j=1}^{3} D_{i j} \mu v_{j}+\sum_{j=1}^{3} \frac{C_{i j}}{2} \rho|v| v_{j}\right)
$$

In equation (14), the first term on the right hand side corresponds to viscous momentum loss in the porous region, and the second term corresponds to inertial losses. In low velocity, laminar flows, the intertial loss term is negligible. Eliminating this term and rewriting the first term based on Darcy's Law gives the simplified equation for simple homogenous porous media. 


$$
S_{i}=-\frac{\mu}{\alpha} v_{i}
$$

In this form of the equation, $\alpha$ is the Darcy permeability. The higher the viscosity of the liquid, or the smaller the permeability, the greater the viscous loss term. The Darcy permeability value for alginate hydrogels was characterized by Hwang et. al. (2010), and found to have a value of $1.2 \pm 0.1 \times 10^{-12} \mathrm{~cm}^{2}$. This is the value used for all studies presented in this thesis.

\section{E. Dimensionless Parameters}

When describing mass transport, it is convenient to use certain dimensionless parameters to describe the relative contributions of advective and diffusive transport. In most CFD studies on similar systems, the Sherwood and Péclet numbers are commonly employed. These quantities are functions of the Reynolds and Schmidt numbers, thus those must be defined first before using them to calculate Sherwood and Peclet numbers.

Perhaps the most ubiquitous of the dimensionless quantities, the Reynolds number gives the ratio of inertial forces to viscous forces in a fluid flow. This is useful not only in defining the dimensionless quantities useful for mass transport, but in verifying the flow regime within the growth chamber. At low Reynolds numbers, viscous forces predominate, and the fluid streamlines are smooth and unidirectional. At high Reynolds numbers, intertial forces predominate, and swirling pockets of fluid known as eddies begin to form. According to McCabe et. al., the Reynolds number in a packed bed is defined as follows:

$$
R e=\frac{\text { Viscous Forces }}{\text { Inertial Forces }}
$$




$$
R e=\frac{D_{p} V_{s} \rho}{(1-\varepsilon) \mu}
$$

The Reynolds numbers calculated for each of the cases simulated are reported in the results section. For a packed bed, $\operatorname{Re}<10$ indicates laminar flow, while $10 \leq \operatorname{Re} \leq 2000$ corresponds to the transition regime. Re $>2000$ indicate fully turbulent flow. Because of the relatively low flow rate, all of the cases simulated fall within the laminar regime, or very low in the transition regime. This is sufficient justification for using the laminar flow model for all CFD simulations.

Similar to the Reynolds number, the Schmidt number relates the momentum diffusivity, otherwise known as viscosity, to the mass diffusivity. It is a quantitative measure of the relative ease of momentum and mass transport. The Schmidt number is not dependent directly on flow conditions, but is instead a property of the mixture. It is defined as follows:

$$
S c=\frac{\mu}{\rho D}
$$

The Sherwood number and Peclet number are closely related quantities. Both are the ratio of two mass transport rates. The Peclet number is dependent upon a characteristic length, L, and gives the ratio of the advective transport rate to the diffusive transport rate. A Peclet number greater than 1 indicates predominantly advective transport, while a Pelcet number less than 1 indicates that diffusion is the primary means of mass transport.

$$
\begin{gathered}
P e=\frac{\text { Rate of Advective Transport }}{\text { Rate of Diffusive Transport }} \\
P e=\frac{L u}{D}=R e * S c
\end{gathered}
$$


The Sherwood number, also a function of the Reynolds and Schmidt numbers, gives the ratio of total mass transfer rate to the rate of diffusion alone.

$$
\begin{gathered}
S h=\frac{\text { Rate of Total Mass Transfer }}{\text { Rate of Mass Transfer by Diffusion }} \\
\qquad h=\frac{K L}{D}
\end{gathered}
$$

The Sherwood number is related to the Reynolds and Schmidt numbers by the Froessling correlation:

$$
S h=2+0.552 R e^{1 / 2} S c^{1 / 3}
$$

\section{F. Examples of Similar Studies Using CFD}

Though the application of CFD to a packed bed bioreactor for tissue engineering is novel, numerous studies have been conducted using CFD for both packed bed reactors and bioreactors of other geometries. The techniques outlined in these studies proved useful in the development of the TPS model. This section is meant as a brief synopsis of some of the key studies used to develop the TPS model.

The most similar study to the one in this thesis is by Baker (2011). Baker studied the effects of packing regime on pressure drop inside of packed beds and found a significant correlation between the tortuosity of the void spaces and the pressure drop simulated in CFD. Baker also used a similar method to Mughal et. al. to generate the geometry for his models, utilizing a Monte Carlo 
algorithm to simulate close packing with analogous results. Using this method, Baker highlighted a key concern also experienced when modeling the TPS. Due to the nature of the close contact points between the spheres, highly skewed cells are often generated during the meshing process. Baker reports that despite poor mesh elements, the solutions still offer a good approximation of the flow field, and offers strategies to mitigate the inaccuracies caused by poor mesh elements. Duscha et. al. (2012) also outlined several approaches for dealing with this issue, including shrinking the diameter of the spheres such that the spheres are not in direct contact, enlarging the diameter of the spheres such that they overlap slightly, or bridging the contact points with a small cylindrical appendage. For the TPS model, the second approach was taken, due to its ease of implementation in CAD and its minimal effect on void fraction within the column.

A number of studies using CFD for bioreactors have focused on the relationship between permeability and nutrient transport. Ma et. al. (2007) studied the effects of altering the permeability of the scaffold material in a perfused bioreactor system with a rotating wall. This study highlighted the improvement of nutrient transport with increased permeability. Fan et. al. (2013) also discuss the relationship between permeability and nutrient transport. Using histological analysis, they found that increased scaffold permeability led to greater differentiation and mineralization in bone tissue. Though alginate has been used in all trials thus far in the TPS, the CFD model makes it simple to study what would happen if more permeable materials were used as a scaffold. Additionally, Eiselt et. al. (2000) outline methods for generating more porous alginate hydrogels by incorporating gas bubbles when crosslinking the gels, thus allowing the alginate permeability to be tunable. Part of the data generated for this thesis involves testing different permeability values to study the magnitude by which nutrient transport is affected.

Since shear stress has been widely recognized as an important factor in bone tissue 
engineering and other applications as well, it is not surprising that most CFD models for bioreactors are designed primarily to study shear stress distributions. Anisi et. al. (2014) and Lawrence et. al. (2009) both modeled a bioreactor system with a flat channel as a growth chamber. Though the geometries differed, both pointed out phenomena that lead to uneven shear distribution in the reactor. Dependent on the flow rate and placement of the inlets and outlets to the growth chamber, "channeling" can occur, whereby the majority of the fluid is confined to a small region with high velocity, where shear is at a maximum. Consequently, the formation of "dead zones" also occurs, where flow stagnates and shear is very small. This situation is analogous to TPS configurations described in the preceding sections where the $\mathrm{D} / \mathrm{d}$ ratio is such that all beads are in contact with the wall of the growth chamber. This allows a channeling effect to take place, whereby the majority of fluid flows through the central channel, leading to an uneven shear distribution.

Truscello et. al.extended the CFD analysis of shear stress to a growth chamber consisting of square pillars of scaffold material laid out in a grid. Velocity profiles were generated in the channels between the pillars and wall shear along the walls of the pillars was analyzed. This study also used oxygen tension to determine the maximum size of the pillars such that oxygen saturation occurs throughout. The ultimate goal of the TPS model is the upscaling of the TPS to a clinically relevant size. Though the capability to study oxygen saturation has not yet been added to the TPS model, this is a promising future direction for improvement.

Because of the novelty of the TPS design, the only existing literature over modeling of the TPS is the 2D COMSOL model developed by Fisher et. al. and described in previous sections. Though the other studies summarized here all differ widely in geometry, the methods used by other researchers lend confidence that the TPS model is valid and useful. 


\section{EXPERIMENTAL METHODS}

\section{A. Geometry Generation}

The first step in the simulation of the bioreactor growth chamber is recreating the geometry of the chamber using 3D computer aided design. Positioning the beads to replicate the tight packings simulated by Mughal et. al. (2012) is not straightforward except in the simplest of cases, as the patterns are often complicated and difficult to visually identify. The simulated annealing approach used by Mughal, as outlined in the previous section, is not easily implemented in CAD programs and requires significant simulation time. For the purposes of this study, an alternative method was developed using SolidWorks 2014-2015 and the built-in motion study utilities. The basic process by which the geometry files were created involves placing the beads above the chamber, fixing the position of the chamber in space, and allowing simulated gravity to settle the beads few at a time to ensure no stacking faults are present. Once it has been verified that the stacking of the beads matches that predicted by Mughal, the beads are fixed in place and the process is repeated until the column of beads has reached the desired height. In this study, the target height for all of the columns was $30 \mathrm{~mm}$, or approximately $23 \%$ of the $130 \mathrm{~mm}$ tube comprising the growth chamber. Once all of the geometries were generated, the void fractions were determined and compared with those predicted by Mughal for validation. A step-by-step description of the geometry generation process follows. 


\section{Create the Bead Part}

Open a new part file in SolidWorks. Create a semicircular sketch in any plane. Define the desired radius by clicking the arc and typing the dimension in the side panel, or using the Smart Dimension tool. Generate the 3D part from the sketch by using Features $>$ Revolved Boss/Base, selecting the arc as the contour and the line segment as the axis of revolution. Set the revolution angle to $360^{\circ}$. Save this part file.

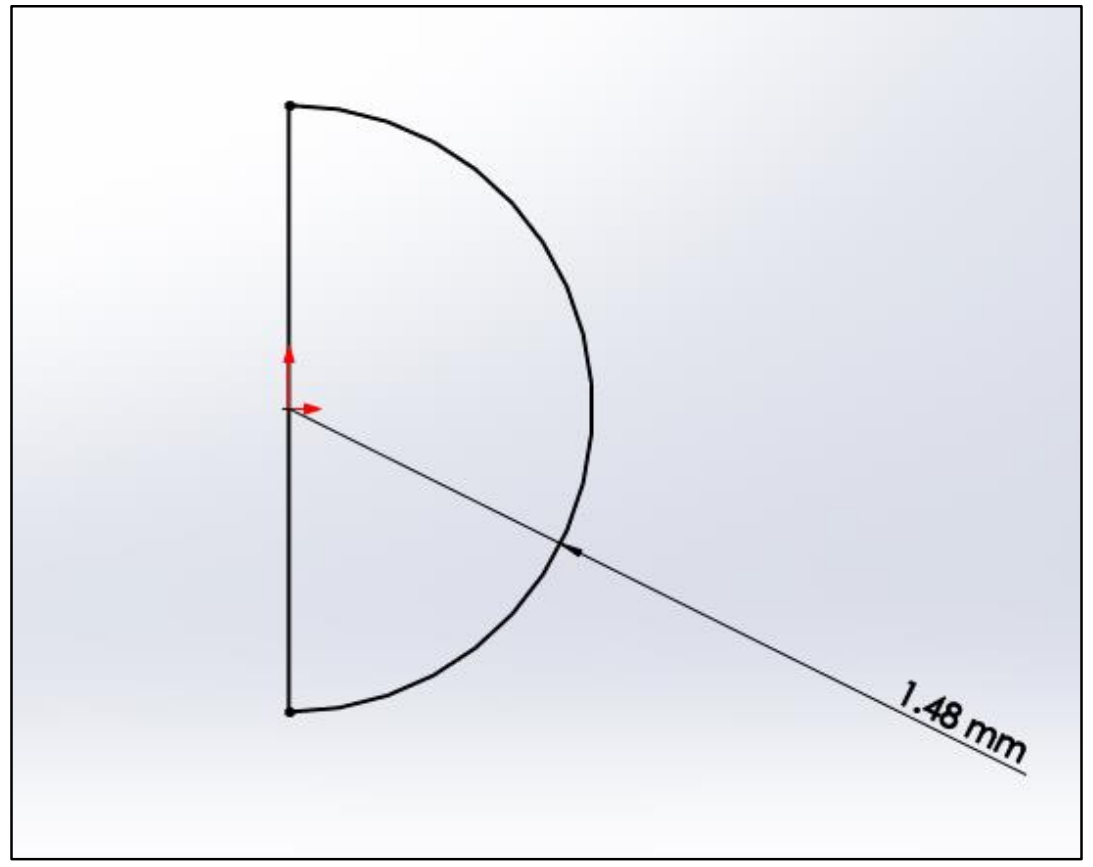

FIGURE 8 - Semicircular sketch used to generate spherical bead 

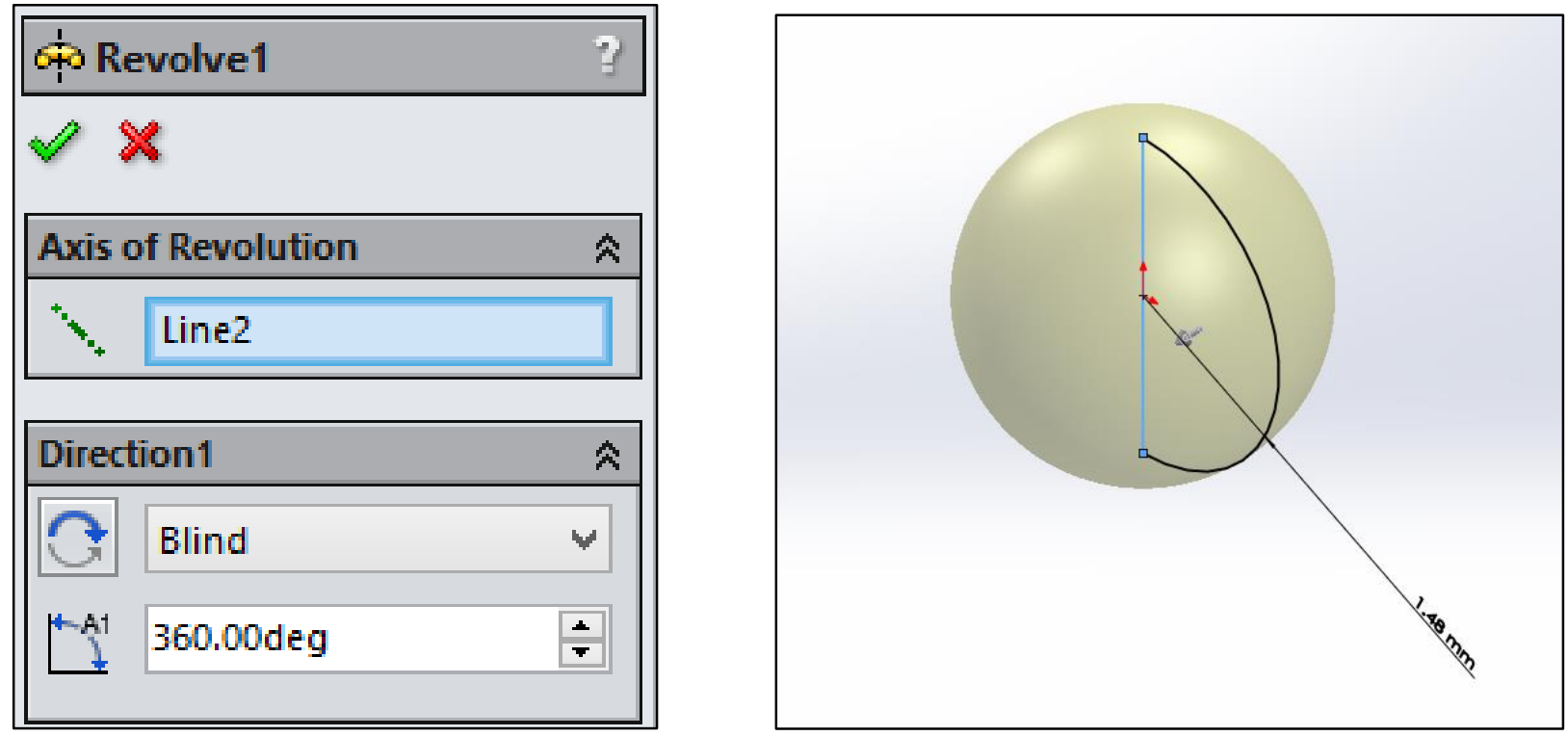

FIGURE 9 - Revolve feature dialog box (left) and finished bead with sketch visible (right)

\section{Create the Growth Chamber Part}

The dimensions of the growth chamber simulated in this study are $130 \mathrm{~mm}$ in length by 6.35 mm inner diameter. In CFD, the geometry represents only the area where the fluid resides, thus the outer diameter of the growth chamber is irrelevant for this application. A solid cylinder is first created by drawing a circular sketch in the "top" plane, then extruding it out to $129 \mathrm{~mm}$ using Features > Extruded Boss/Base. To facilitate the next step, where the beads are added to the chamber, the Feature > Shell command is used to create a hollow cup into which the beads can fall. For this feature, the user must select the thickness of the shell and the face for the opening. SolidWorks is unable to handle zero-thickness geometry, so a nonzero value must be entered for 
the thickness of the shell. Through trial and error, it was found that a value of $0.11 \mathrm{~mm}$ gives the best results. Due to the tolerances of the physics solver, thinner walls allow the beads to penetrate the walls and exit the cup during motion simulation. At a value of $0.11 \mathrm{~mm}$, the beads penetrate the walls just enough to be tangent to the exterior boundary of the boundary of the cup without falling out, thus accurately replicating a $6.35 \mathrm{~mm}$ diameter chamber. Once the cavity has been created, use the extrude feature once again to extrude the original circular sketch $1 \mathrm{~mm}$ in the opposite direction, beyond the cavity. This creates a small "outlet zone" which keeps the beads from being directly tangent to the outlet. This serves to improve mesh quality while having a negligible effect on results, as the total overall length is retained at $130 \mathrm{~mm}$.
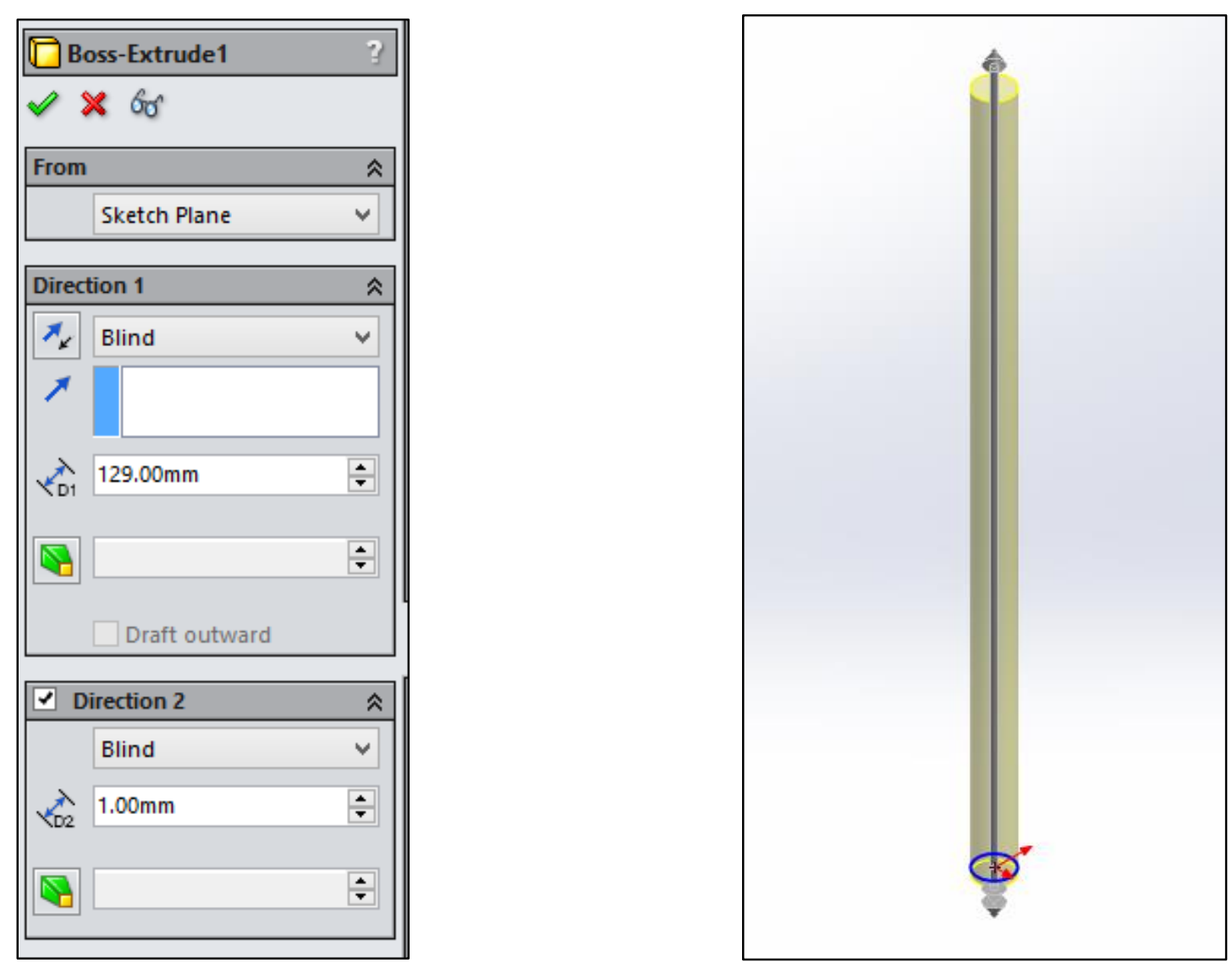

FIGURE 10 - Extruded Boss/Base dialog box (left) and extruded chamber (right) 


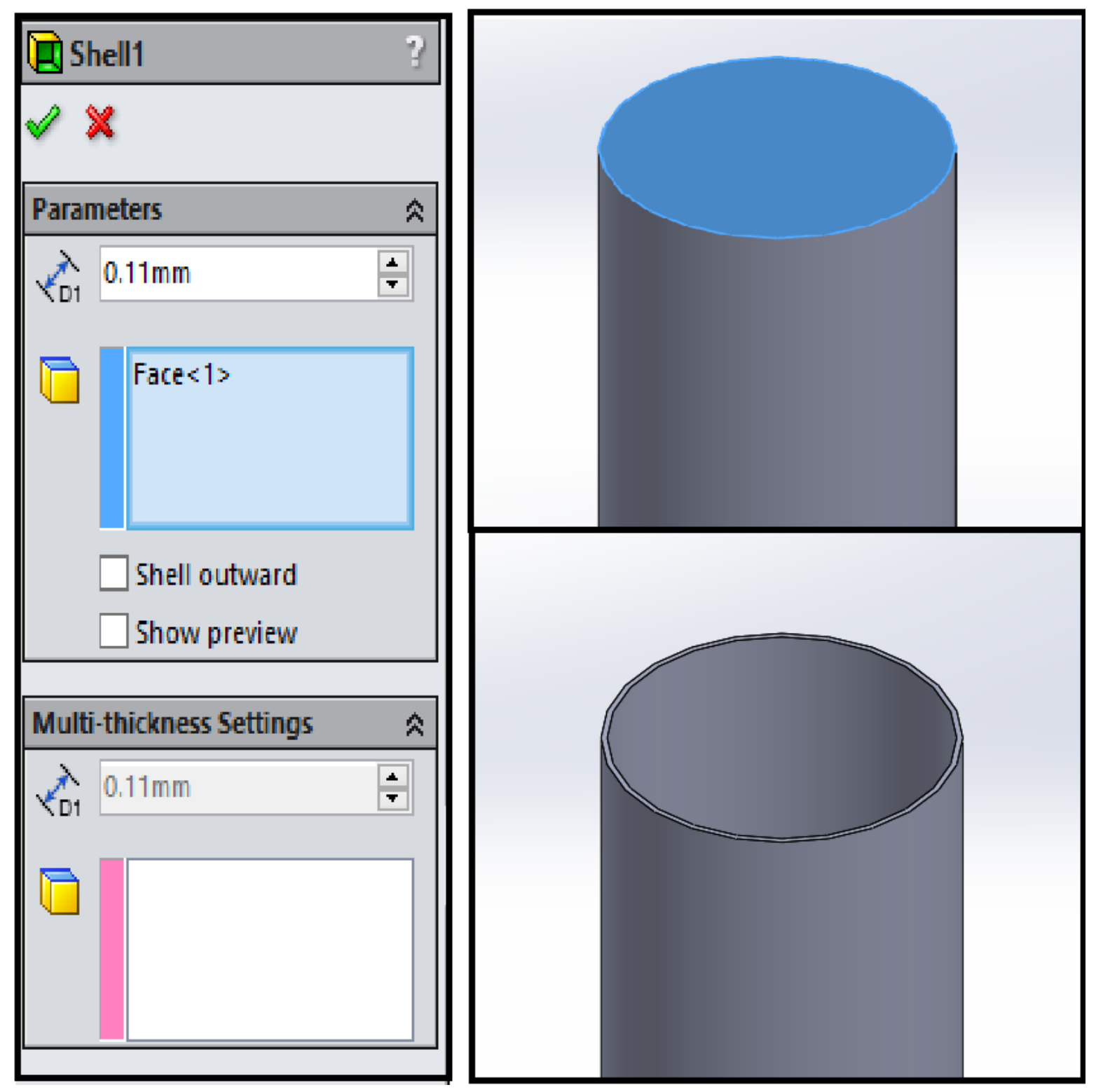

FIGURE 11 - Shell feature dialog box (left), Face selection for shell opening (top right),

Completed shell (bottom right) 


\section{Create the Growth Chamber in a SolidWorks Assembly File}

To unify the bead and chamber parts, begin a SolidWorks assembly file by selecting File New > Assembly. Place the chamber first by selecting the chamber part file and using the green check mark button to paste the part into the assembly. By default, this will place the bottom of the chamber at the origin and fix it in space. To place the beads, use Assembly > Insert Components and select the bead part file. Using the cursor, the beads can be placed anywhere in space, and by default are set to "float" rather than "fixed". It is necessary that the beads are set to "float" and the chamber is set to "fixed" for the gravity simulation to work properly. This should happen by default, but can be verified by right clicking any part and clicking "fix" or "float" to toggle between the two. Since it is important to replicate the structures predicted by Mughal as closely as possible, beads are annealed $4-5$ at a time in order to prevent stacking faults.
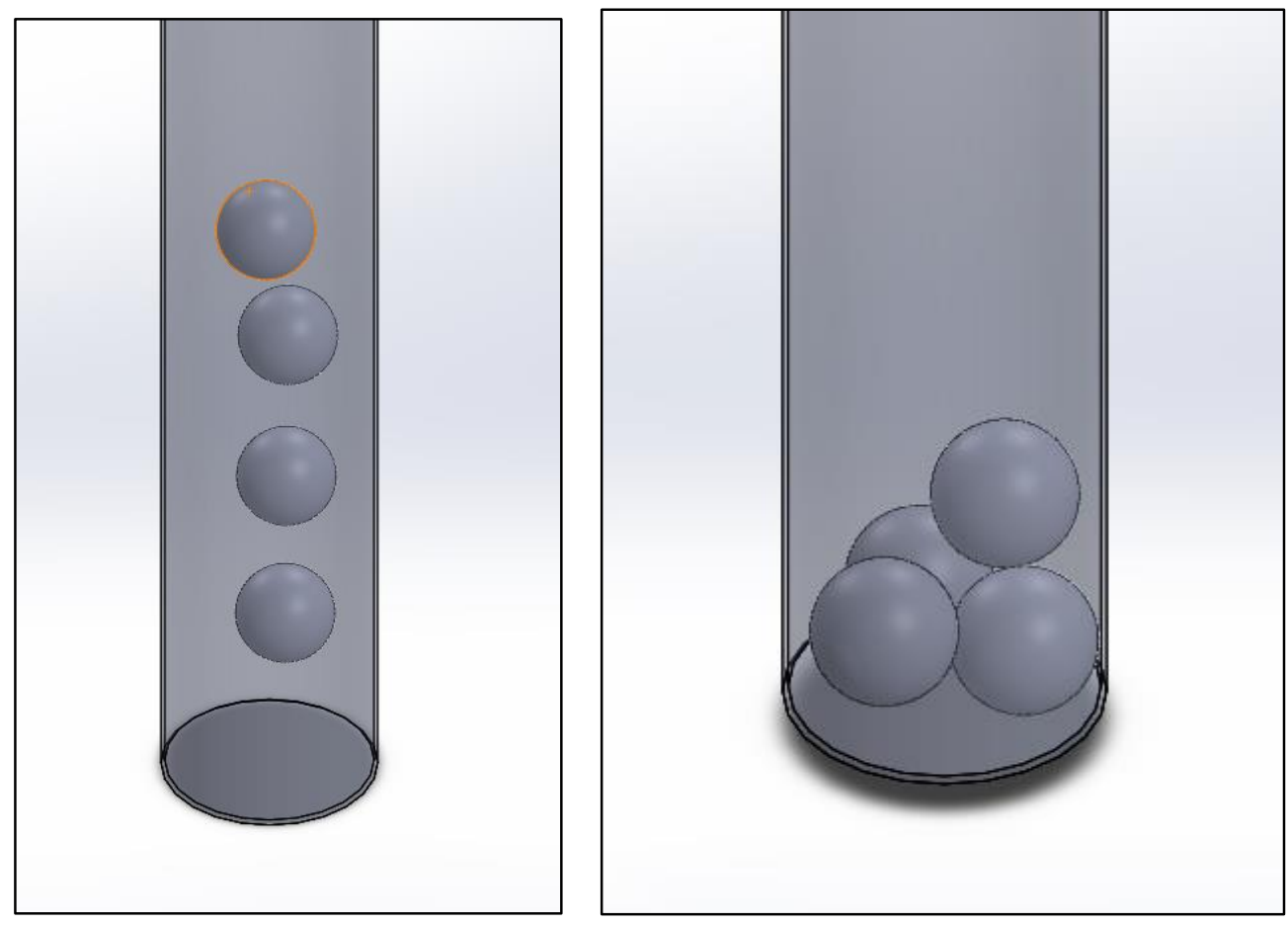

FIGURE 12 - Four beads ready for annealing (left) and after the annealing process (right) 


\section{Running the Motion Study}

Once the beads to be annealed have been placed, a motion study must be created using Assembly $>$ New Motion Study. First change the drop down box in the lower left from "Animation" to "Basic Motion." This enables the use of the physics engine within SolidWorks and will allow the motion simulation to be carried out. Two settings are needed before running the simulation - simulated gravity and simulated contact. Select the apple icon to bring up the gravity dialog box. Ensure that gravity is directed in the downward y direction. A green arrow in the simulation window will show the direction in which gravity will act. For the simulated contact button, select the contact button from the simulation panel. Highlight all of the parts in the model window to simulate solid body contact between all parts. Once these parameters have been set, the simulation is ready to run. Click "Calculate" to begin the simulation. The beads should fall into the cup and settle against each other. If stacking faults are observed, the simulation can be run again using higher gravity values until the beads settle in their proper configuration. Once this is accomplished, the beads can be fixed into place by selecting them in the simulation window and using Right Click > Form New Subassembly. The beads are now frozen in position and ready for the addition of more beads in the modeling window. This motion study can be deleted and the process repeated until the desired column height is reached. 


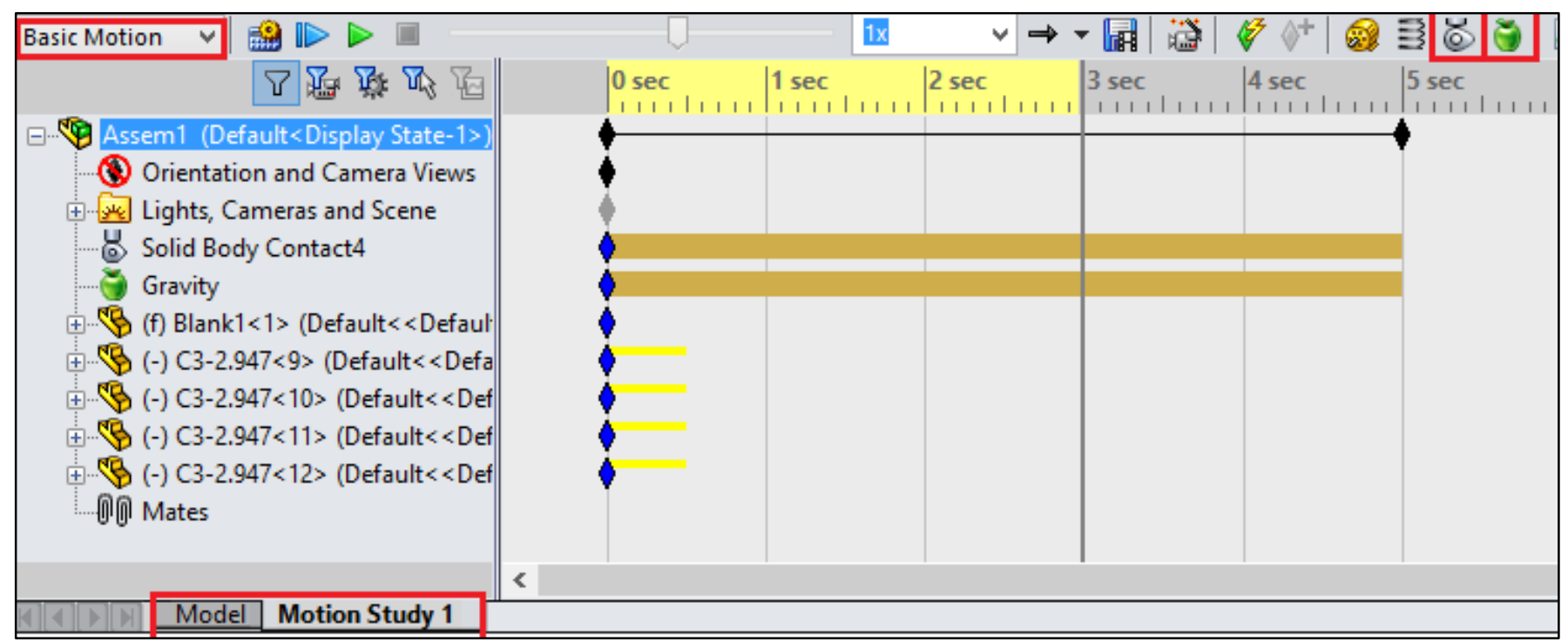

FIGURE 13 - Motion simulation panel with relevant option boxed in red
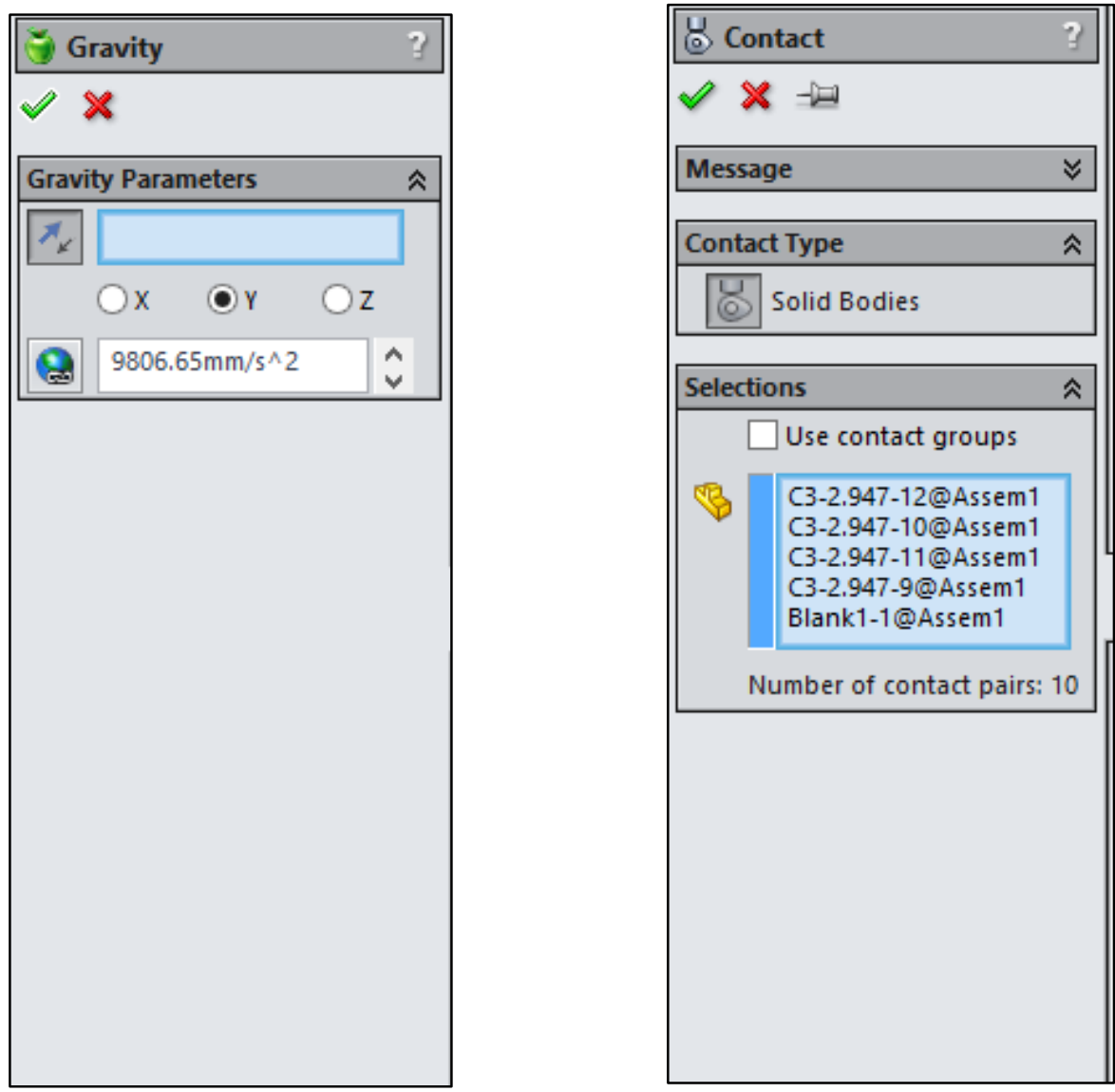

FIGURE 14 - Gravity dialog box (left) and contact dialog box (right) 


\section{Verifying the Height and Volume Fraction}

Once the chamber has been completed, a measurement of the height and volume fraction can be carried out to ensure that the values align closely with the targets. Since beads may only be added in discrete numbers, the target column height of $30 \mathrm{~mm}$ is an approximation. Beads can be added or deleted from the column as appropriate to come as close to $30 \mathrm{~mm}$ as possible. In order to measure the height of the bead column, two parallel planes are generated, one tangent to the top bead in the column and the other tangent to the bottom bead in the column. This is accomplished by using Assembly > Reference Geometry > Plane and using the dialog box to define the plane as tangent to the bead and parallel to the "Top (XZ)" plane. The distance between these two planes can then be measured by selecting the two planes and using Tools > Measure.

Determining the volume fraction of the generated column requires use of the planes generated in the previous step. Because the top and bottom portions of the column often contain an incomplete unit cell, a more accurate measurement of the volume fraction of the column can be obtained by measuring only the interior portion. Thus, the first $5 \mathrm{~mm}$ of each end are "sliced off". First the chamber part is suppressed temporarily using Right Click > Suppress. This leaves only the beads. Using the planes generated in the last step, create a circular sketch larger than the column diameter on each plane and use Features > Extruded Cut to remove $5 \mathrm{~mm}$ from each end of the column. To determine the volume of the beads remaining, use Tools > Mass Properties, which will automatically calculate the volume. Knowing the height of the column, determining the chamber volume and volume fraction is trivial from this point forward. The column can be reverted to its original condition by deleting the cut features and unsuppressing the chamber part. 


\begin{tabular}{|c|c|c|}
\hline$\otimes P$ & ane & ? \\
\hline \multicolumn{3}{|c|}{$\mathscr{X}-\not$} \\
\hline Mes: & iage & $\hat{\imath}$ \\
\hline \multicolumn{3}{|c|}{ Fully defined } \\
\hline \multirow{2}{*}{\multicolumn{3}{|c|}{ First Reference }} \\
\hline & & \\
\hline \multicolumn{3}{|c|}{ () Tangent } \\
\hline \multicolumn{3}{|c|}{$\square$ Flip } \\
\hline \multicolumn{3}{|c|}{ Second Reference } \\
\hline & Top Plane & \\
\hline & Parallel & \\
\hline$\perp$ & Perpendicular & \\
\hline 久 & Coincident & \\
\hline 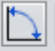 & $90.00 \mathrm{deg}$ & $\hat{a}$ \\
\hline$\mapsto$ & $10.00 \mathrm{~mm}$ & $\hat{a}$ \\
\hline & Mid Plane & \\
\hline
\end{tabular}

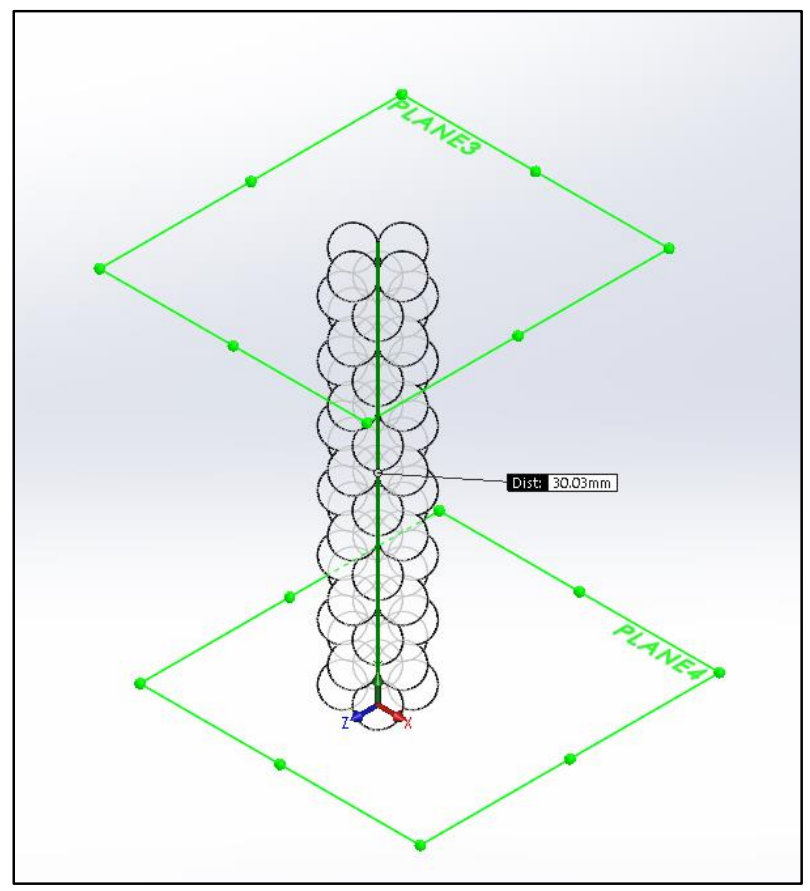

FIGURE 15 - Plane creation dialog box (left) and reference planes (right)

\begin{tabular}{|c|c|c|}
\hline \multicolumn{3}{|c|}{$\begin{array}{l}\text { Mass properties of MC3-1 fixed } \\
\text { Configuration: Default } \\
\text { Coordinate system: -- default -- }\end{array}$} \\
\hline \multicolumn{3}{|l|}{ Mass $=0.32$ grams } \\
\hline \multicolumn{3}{|c|}{ Volume $=319.59$ cubic millimeters } \\
\hline \multicolumn{3}{|c|}{ Surface area $=709.99$ square millimeters } \\
\hline $\begin{array}{l}\text { Center of mass: ( millimeters } \\
\qquad \begin{array}{l}X=-0.01 \\
Y=15.09 \\
Z=0.01\end{array}\end{array}$ & & \\
\hline $\begin{array}{l}\text { Principal axes of inertia and } \\
\text { Taken at the center of mass. } \\
\qquad \begin{array}{l}\mid x=(0.00,1.00,-0.00) \\
\mid y=(-0.77,0.00,0.64) \\
\mid z=(0.64,-0.00,0.77)\end{array}\end{array}$ & $\begin{array}{l}\text { rincipal mom } \\
\qquad \begin{array}{l}P x=1.27 \\
P y=11.02 \\
P z=11.07\end{array}\end{array}$ & ( grams * square $n$ \\
\hline \multicolumn{3}{|c|}{$\begin{array}{l}\text { Moments of inertia: ( grams }{ }^{*} \text { square millimeters) } \\
\text { Taken at the center of mass and aligned with the output coordinate system. }\end{array}$} \\
\hline $\begin{array}{l}L x x=11.04 \\
L y x=0.03 \\
L z x=-0.02\end{array}$ & $\begin{array}{l}L x y=0.03 \\
L y y=1.27 \\
L z y=-0.02\end{array}$ & $\begin{array}{l}L x z=-0.02 \\
L y z=-0.02 \\
L z z=11.05\end{array}$ \\
\hline $\begin{array}{l}\text { Moments of inertia: ( grams } \\
\text { Taken at the output coordin } \\
\qquad \begin{array}{l}\mid \mathrm{xx}=83.82 \\
\text { Iyx }=-0.00 \\
\mid \mathrm{zx}=-0.02\end{array}\end{array}$ & $\begin{array}{l}\text { square millin } \\
\text { te system. } \\
\begin{array}{l}\mid x y=-0.00 \\
\mid y y=1.27 \\
\mid z y=0.01\end{array}\end{array}$ & $\begin{array}{l}\mid x z=-0.02 \\
\mid y z=0.01 \\
\mid z z=83.83\end{array}$ \\
\hline
\end{tabular}

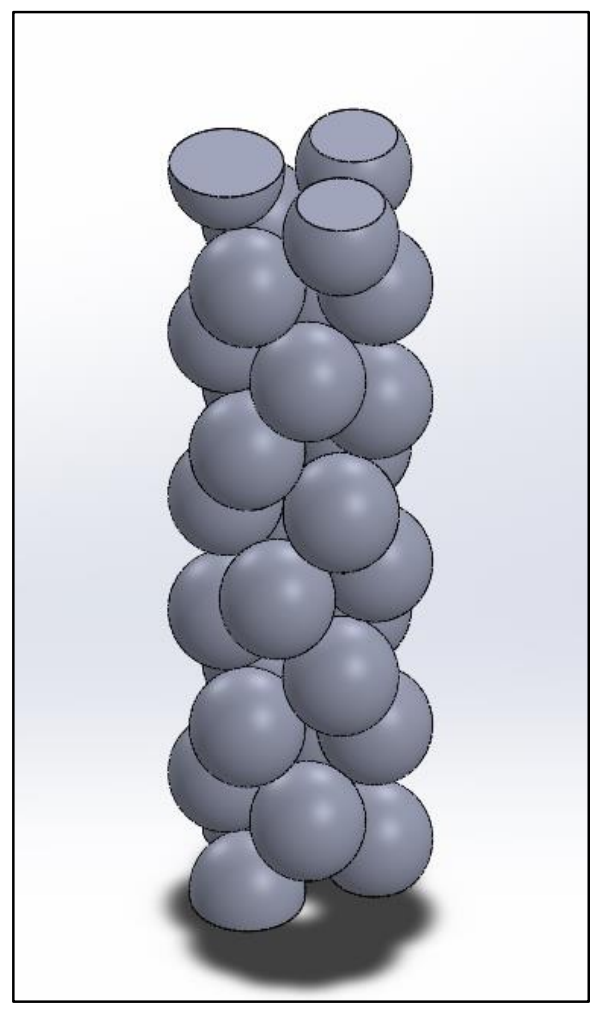

FIGURE 16 - Mass properties data (left) and trimmed column for volume fraction determination (right) 


\section{B. Mesh Generation}

Begin a new fluent project in ANSYS Workbench. Import the assembly file by right clicking the geometry cell and selecting "Import File." Ansys has the native capability to handle SolidWorks Assembly (.SLDASM) files, so no pre-processing should be necessary. A green check will appear in the geometry cell if the file import is successful. Open ANSYS meshing by double clicking the "Mesh" cell. Prior to meshing, named selections should be created in order to denote the important features of the geometry. This is accomplished by selecting the surface or body to be named and using Right Click > Create Named Selection. The named selections for this study are "velocity-inlet" for the top surface of the chamber, "pressure-outlet" for the bottom surface, "tube-wall" for the outer wall of the chamber and "beads" for all of the beads.

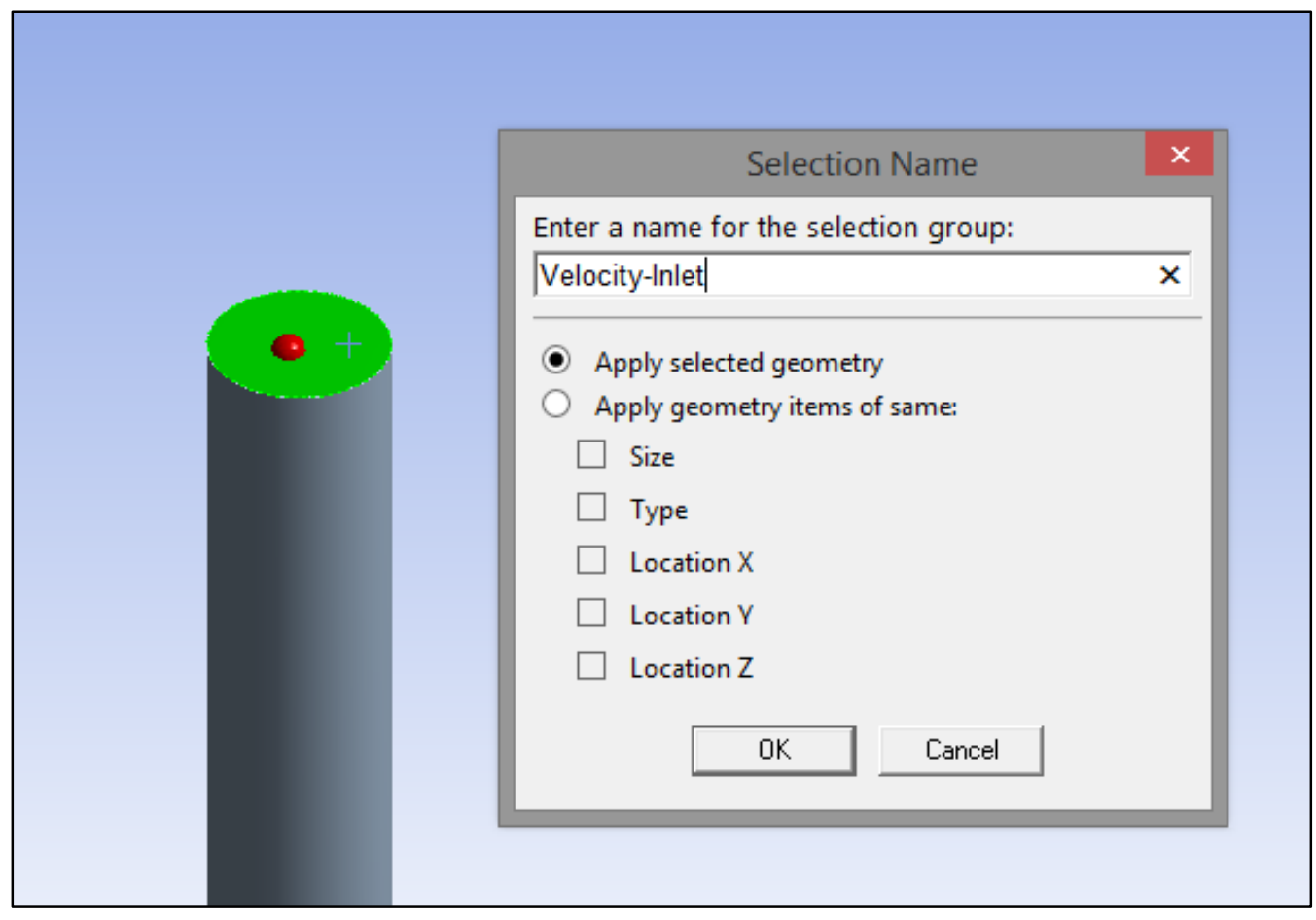

FIGURE 17 - Named selection creation dialog for inlet surface 
Once the named selections have been set, the mesh generation can be initiated. Typically in geometries which contain multiple zones for fluid flow, such as the hollow chamber and the porous beads, boolean operations must be manually performed to define the different zones. As an alternative for this study, the assembly meshing algorithm is used to automatically identify and separate the mesh zones. Of the two possible assembly meshing algorithms, CutCell was determined to yield better quality mesh and promote better solution convergence. This method creates a hexahedral dominant mesh, which is well suited to long sections of pipe. Tetrahedral meshing, on the other hand, tends to produce degenerate elements at the contact points, resulting in poor convergence. The advanced size feature is used to improve the resolution at close contact points. The mesh parameters are set using the following commands:

- Assembly Meshing > Method > CutCell

- Sizing > Advanced Size Function > On: Proximity and Curvature

- $\quad$ Sizing $>$ Relevance Center $>$ Fine

- Defaults $>$ Relevance $>100$

- Sizing > Max Size $>0.1 \mathrm{~mm}$ 


\begin{tabular}{|l|l|l|}
\hline Defaults \\
\hline Physics Preference & CFD \\
\hline Solver Preference & Fluent \\
\hline \multicolumn{1}{|l|}{ Relevance } & 100 \\
\hline Sizing & \\
\hline Use Advanced Size Function & On: Proximity and Curvature \\
\hline Relevance Center & Fine \\
\hline Smoothing & Medium \\
\hline$\square$ Curvature Normal Angle & Default (12.0 $)^{\circ}$ \\
\hline$\square$ Proximity Accuracy & 0.5 \\
\hline$\square$ Num Cells Across Gap & Default (5) \\
\hline Proximity Size Function Sources & Edges \\
\hline$\square$ Min Size & Default (2.0517e-002 mm) \\
\hline$\square$ Proximity Min Size & Default (2.0517e-002 mm) \\
\hline$\square$ Max Size & $0.164130 \mathrm{~mm}$ \\
\hline$\square$ Growth Rate & Default (1.10) \\
\hline Minimum Edge Length & $19.9490 \mathrm{~mm}$ \\
\hline Inflation & \\
\hline Assembly Meshing & \\
\hline Method & CutCell \\
\hline Feature Capture & Program Controlled \\
\hline Tessellation Refinement & \\
\hline Statistics & \\
\hline
\end{tabular}

FIGURE 18 - Meshing dialog box with relevant fields highlighted in red

The program is now ready for mesh generation. Click "Generate" to begin the meshing process. This process takes several minutes. The completed mesh should contain between 1.5-1.7 million elements depending on the bead size being simulated. Based on grid-independence studies, this resolution was found to be adequate to ensure that the solution was not dependent on element count. The following step depends on the type of study being performed. For studies on surface shear, the bead mesh is suppressed, leaving behind a hollow cavity. In this case, Fluent will treat the boundary of the beads as a no-slip boundary where shear can be calculated. If interstitial flow in the beads is to be studied, the bead mesh is not suppressed. When setting up the case, this zone will be denoted as a porous zone in fluent. This method is necessary because Fluent recognizes a 
porous zone boundary as a fluid-fluid boundary, where shear is not calculated. Suppressing the bead mesh can be accomplished by selecting all of the beads in the sidebar, and using Right Click > Suppress.
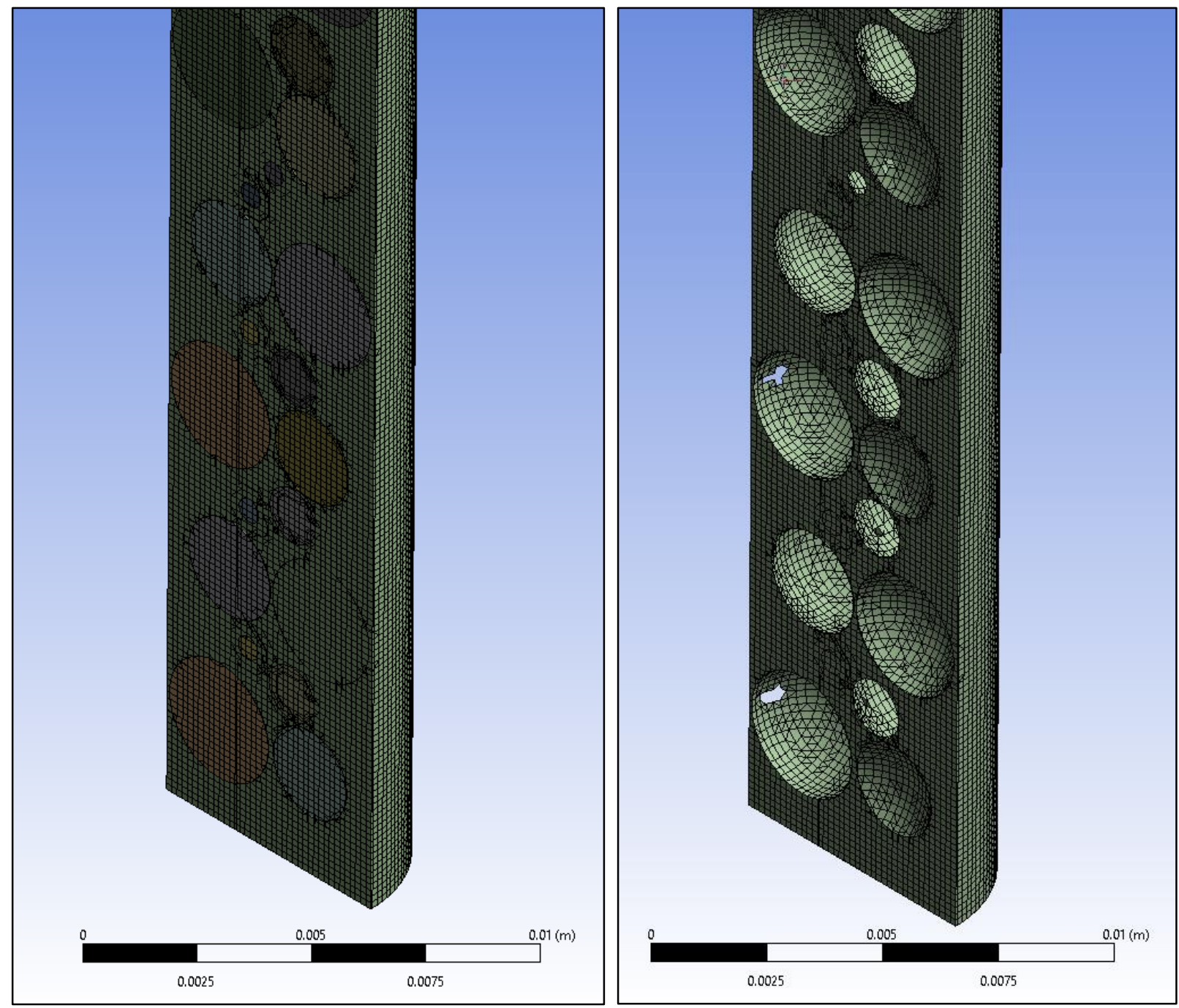

FIGURE 19 - Mesh cross-section with beads unsuppressed (left) and suppressed (right) 
Once the mesh generation is complete, the meshing editor can be closed and the mesh file will be automatically appended to the project in workbench.

\section{Fluent Setup and Simulation}

Once the mesh has been generated, the CFD simulation is ready to be set up. If the mesh has been successfully appended to the project in Ansys workbench, a green check mark will appear in the "Mesh" cell. Bringing up the Fluent interface is accomplished by double clicking the "Setup" cell, which will load the mesh into fluent automatically. The following sequence of steps is necessary in order to set up the simulation.

\section{General Settings}

Ensure that all the general settings are set to the default values as shown.

\begin{tabular}{|c|c|c|c|}
\hline Meshing & \multicolumn{3}{|l|}{ General } \\
\hline Mesh Generation & \multicolumn{3}{|l|}{ Mesh } \\
\hline Solution Setup & Scale... & Check & Report Quality \\
\hline Models & Display... & & \\
\hline $\begin{array}{l}\text { Materials } \\
\text { Phases }\end{array}$ & & \multicolumn{2}{|c|}{ Solver } \\
\hline $\begin{array}{l}\text { Cell Zone Conditions } \\
\text { Boundary Conditions } \\
\text { Mesh Interfaces } \\
\text { Dynamic Mesh } \\
\text { Reference Values }\end{array}$ & $\begin{array}{l}\text { Type } \\
\text { Pressure-Based } \\
\text { Density-Based }\end{array}$ & \multicolumn{2}{|c|}{$\begin{array}{l}\text { Velocity Formulation } \\
\text { Absolute } \\
\text { Relative }\end{array}$} \\
\hline $\begin{array}{l}\text { Solution } \\
\text { Solution Methods } \\
\text { Solution Controls } \\
\text { Monitors }\end{array}$ & \multicolumn{3}{|l|}{$\begin{array}{l}\text { Time } \\
\text { Steady } \\
\text { Transient }\end{array}$} \\
\hline $\begin{array}{l}\text { Solution Initialization } \\
\text { Calculation Activities } \\
\text { Run Calculation }\end{array}$ & $\square$ Gravity & \multicolumn{2}{|r|}{ Units... } \\
\hline $\begin{array}{l}\text { Results } \\
\text { Graphics and Animations } \\
\text { Plots } \\
\text { Reports }\end{array}$ & \multicolumn{3}{|l|}{ Help } \\
\hline
\end{tabular}

FIGURE 20 - General settings with default values 
Ensure that the mesh was interpreted in the proper units. Click "Scale..." to open the scaling dialog box and change both dropdown boxes to "mm." Ensure that the dimensions are correct or scale appropriately.

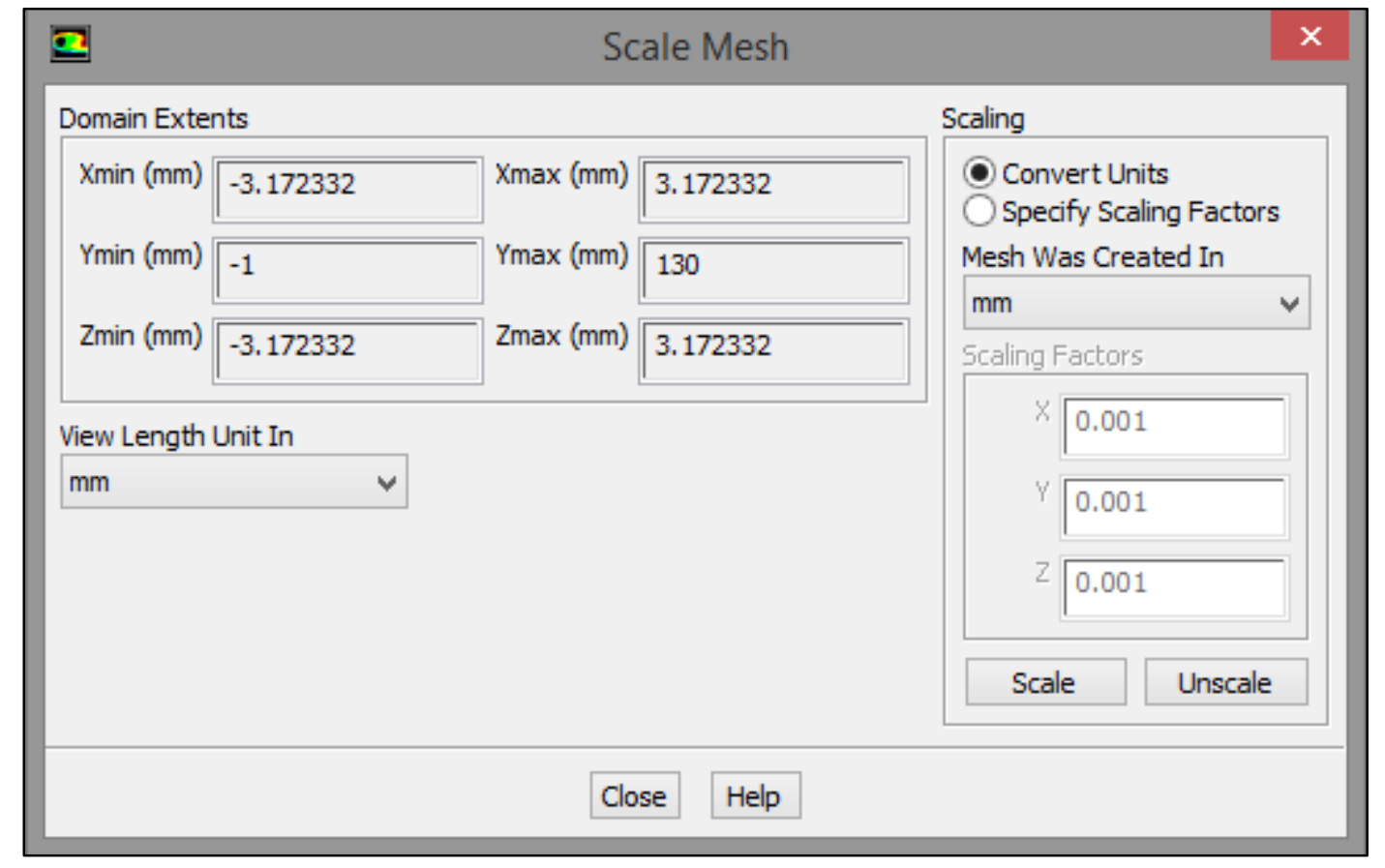

FIGURE 21 - Scale mesh dialog box

\section{Defining the Growth Media Material}

The material properties of the growth media must be defined in Fluent. These values are taken from Fisher et. al. (2011). This is accomplished by clicking Materials > Create/Edit and entering the following parameters:

- Density: 993 kg/m³

- Viscosity: $0.00078 \mathrm{~kg} / \mathrm{m}=\mathrm{s}$

- Name: growth-media 
Once the material properties have been defined, click Change/Create and overwrite "Air" as the default material.

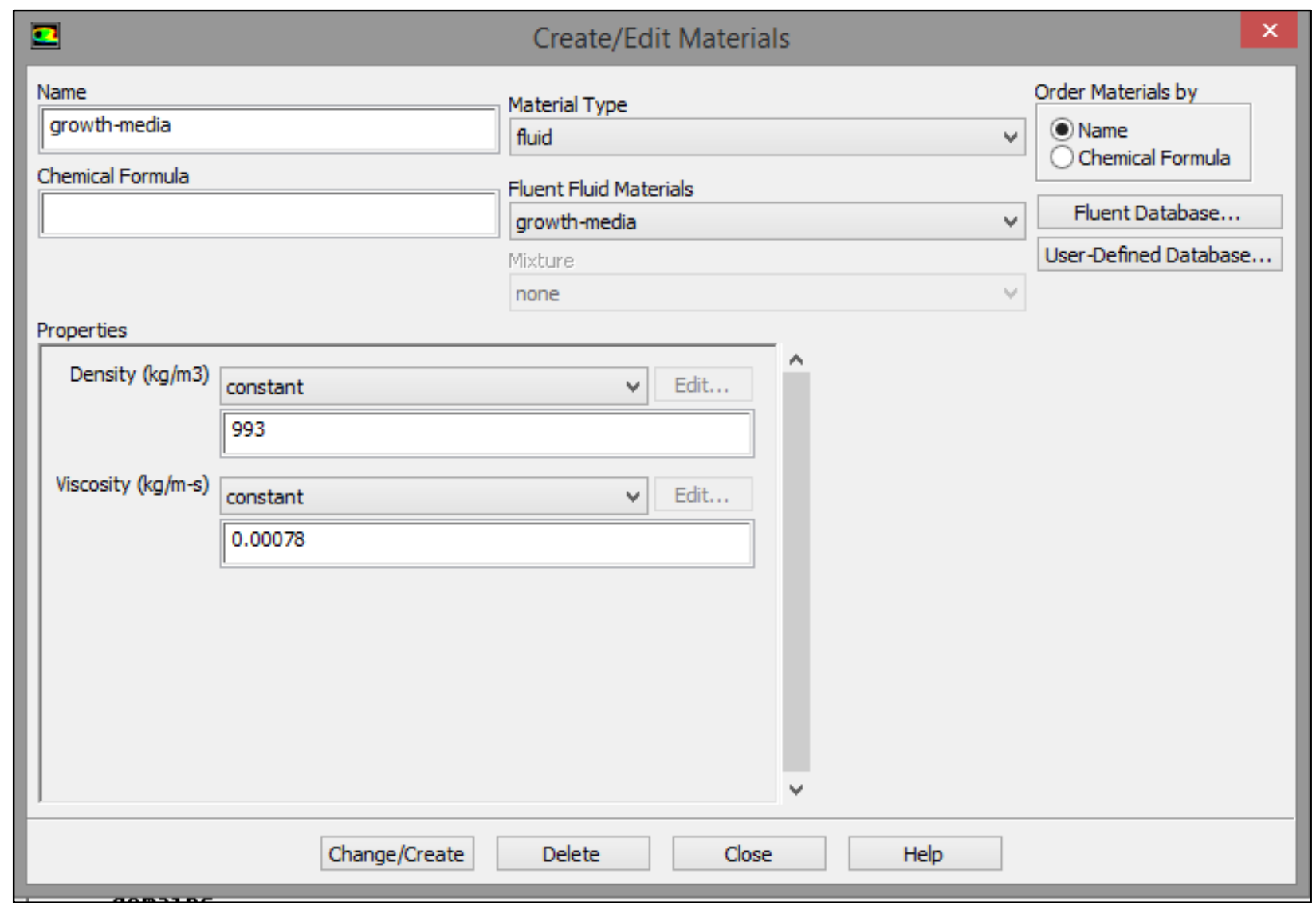

FIGURE 22 - Create/Edit Materials dialog box with correct constants

\section{Setting the Porous Media Parameters using Cell Zone Conditions (Porous Only)}

For shear stress studies in which interstitial flow within the beads is not considered, this step is not applicable. For studies utilizing the porous media model, the properties of the alginate must be defined under "Cell Zone Conditions." As stated in the previous section, the Darcy permeability for sodium alginate has been determined to have a value of $1.2 \times 10^{-12} \mathrm{~cm}^{2}$. The input field for 
permeability in Fluent is termed "Inertial Resistance" and is reported as $1 / \alpha$ rather than $\alpha$. Additionally, the units must be converted from $1 / \mathrm{cm}^{2}$ to $1 / \mathrm{m}^{2}$. Following conversion, the appropriate value is $8.33 \times 10^{15} \mathrm{~m}^{-2}$. The porous media parameters can be accessed using Cell Zone Conditions $>$ Beads $>$ Porous Zone. Since the flow is low velocity and low Reynolds number, the inertial resistance terms are left at the default value of zero. Viscous resistance is considered to be isentropic, thus the same value is used for each Direction-1, Direction-2, and Direction-3 (defined as $\mathrm{X}, \mathrm{Y}$, and Z direction by default).

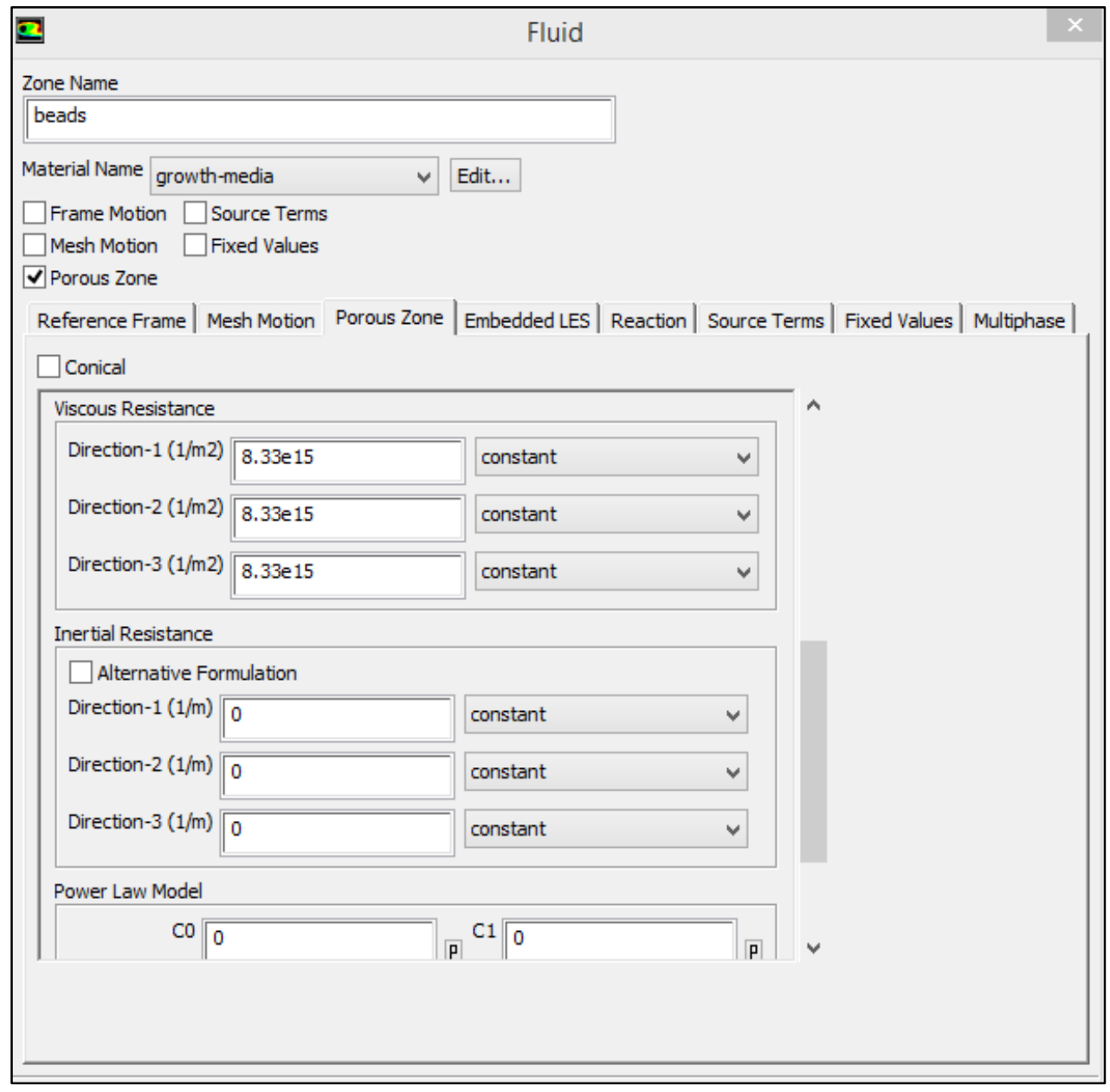

FIGURE 23 - Cell Zone Conditions dialog box with porous zone parameters defined 
In addition to defining the zone permeability, it is critical that the "Porous Formulation" option be set to "Physical Velocity." This can be toggled in the Cell Zone Conditions panel.

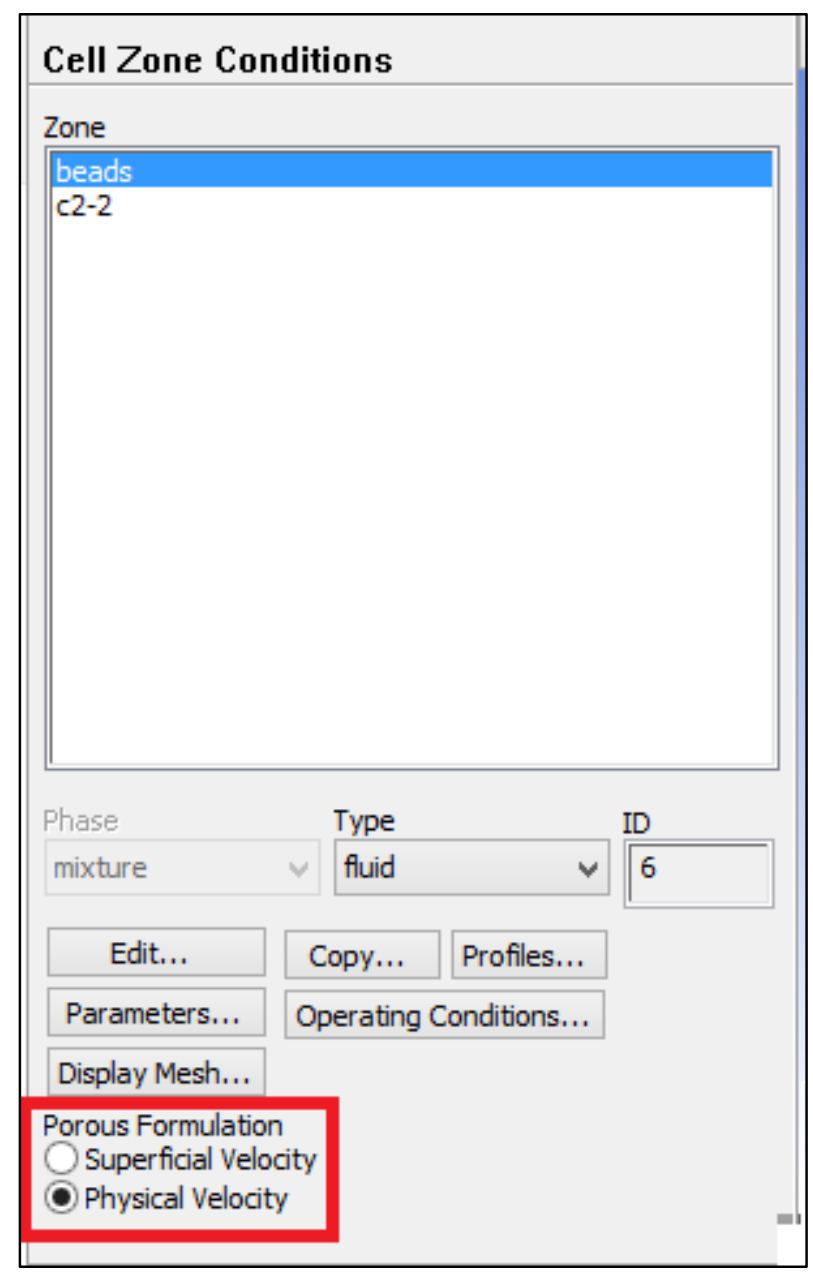

FIGURE 24 - Cell Zone Conditions panel with Physical Velocity Formulation selected 


\section{Setting the Boundary Conditions}

Boundary conditions are used to define inlets and outlets in a flow problem. By creating the two named selections, "Velocity-Inlet" and "Pressure-Outlet," during the mesh generation step, the boundary types should be automatically interpreted by Fluent. A Pressure-Outlet is an outlet boundary defined by the static gauge pressure at the boundary. This value is arbitrary, and by default is set to zero. For the purposes of this simulation, this setting is retained. In order to define the flow rate, the boundary condition for "Velocity-Inlet" must be defined. A flow rate of $3 \mathrm{~mL} / \mathrm{min}$ through a $6.35 \mathrm{~mm}$ diameter circular cross section equates to a flow velocity of 0.0016 $\mathrm{m} / \mathrm{s}$, normal to the surface. This parameter is defined by clicking Boundary Conditions > Velocity-Inlet $>$ Edit... and changing the value of Velocity Magnitude to $0.0016 \mathrm{~m} / \mathrm{s}$.

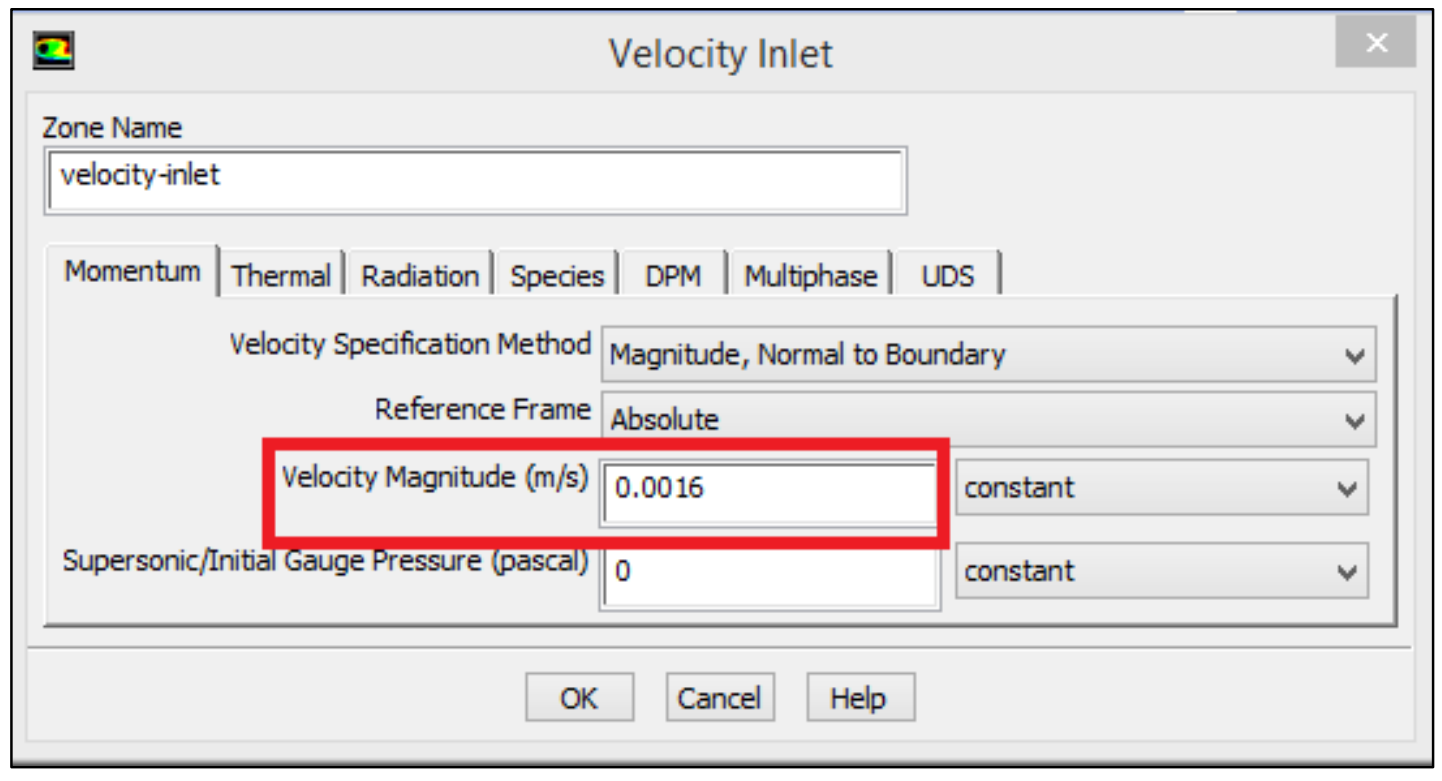

FIGURE 25 - Inlet boundary conditions dialog box 


\section{Setting the Solution Methods}

Solution methods are chosen based on the geometry and flow conditions of the simulation, and will affect the convergence time and robustness of the final solution. In general, the default solution methods are well suited to low Reynolds number, single phase flows with low pressure gradients, such as the simulations presented in this thesis. Therefore, the default solution methods are retained with the exception of the momentum solver, which is changed from first-order to second-order. This method is more computationally intense but allows for a greater degree of accuracy when evaluating surface shear. The solution methods are as follows:

- Pressure-Velocity Coupling Scheme: SIMPLE

- Gradient Discretization Scheme: Least Squares Cell Based

- Pressure Discretization Scheme: Standard

- Momentum Discretization Scheme: $\quad$ Second Order Upwind

\section{Setting the Convergence Monitors}

Convergence monitors must be established to determine solution convergence. By default, residual values are used as a metric for convergence, signifying the imbalance of a conserved quantity within a volume, such as the difference between mass in and mass out. A drop of 2-3 orders of magnitude in residual values followed by a plateau is a good general indicator of convergence. However, residuals only provide a very general indicator of convergence and do not ensure that a solution is accurate. Additional monitors are important to judge convergence. Typically these monitors are chosen to correspond with the variable to be evaluated. In this case, the area-weighted average of surface shear along the wall of the beads is used as a convergence 
monitor. To define this monitor, select Monitors $>$ Surface Monitors $>$ Create and select the surface corresponding to the bead wall. Use Report Type > Area Weighted Average and Field Variable > Wall Fluxes > Wall Shear Stress to define the monitor. Select "Print to Console" and "Plot" so that the value can be monitored while Fluent carries out the iterative solution.

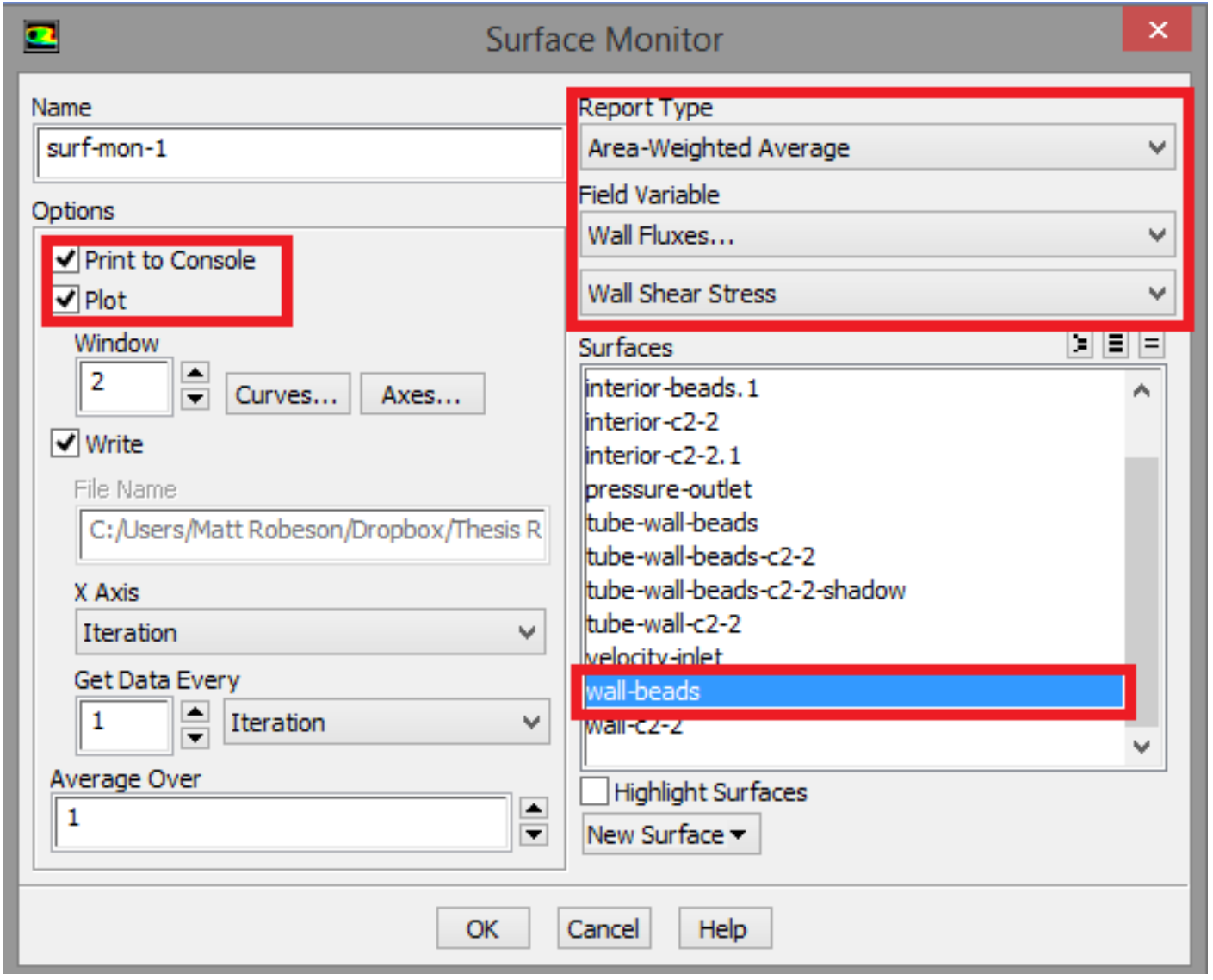

FIGURE 26 - Surface Monitor dialog box with relevant fields highlighted 


\section{Running the Simulation and Judging Convergence}

The simulation is now completely defined and ready to run. Initialization of the solution is carried out by clicking Solution Initialization $>$ Hybrid Initialization > Initialize. This fills the mesh cells with initial guess values for the iterative solution. Once initialized, the simulation can be run using the "Run Calculation" panel. Typically these simulations converge within 100 iterations, therefore the number of iterations is set to 100 . Click "Calculate" to begin the simulation.

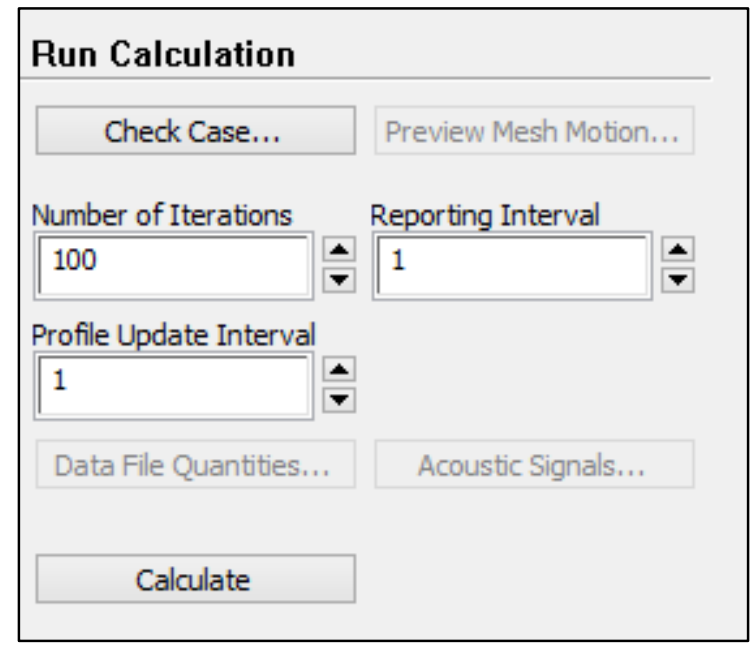

FIGURE 27 - Run Calculation panel with number of iterations set to 100

As the simulation runs, a graphical representation of both the residual values and the surface monitor is available. The drop down box at the top of the window can be used to toggle between the two. In addition, the numerical values for each iteration will print to the console. Convergence is assumed when the residuals have decreased by at least two orders of magnitude and leveled off, and the surface monitor changes by less than $0.01 \%$ between successive iterations. The two figures below illustrate the typical appearance of the monitors when convergence is achieved. 


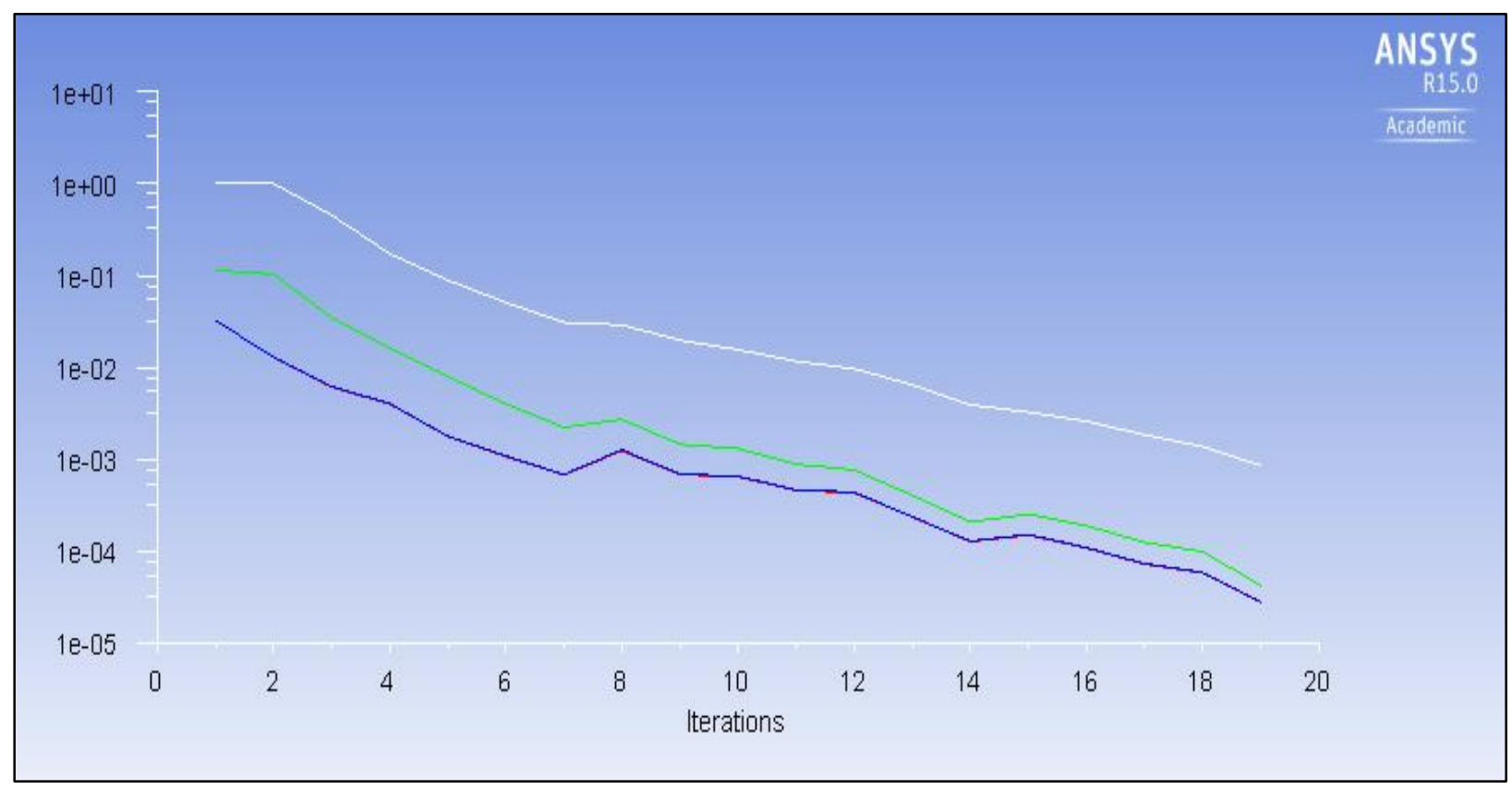

FIGURE 28 - Convergence history of scaled residuals

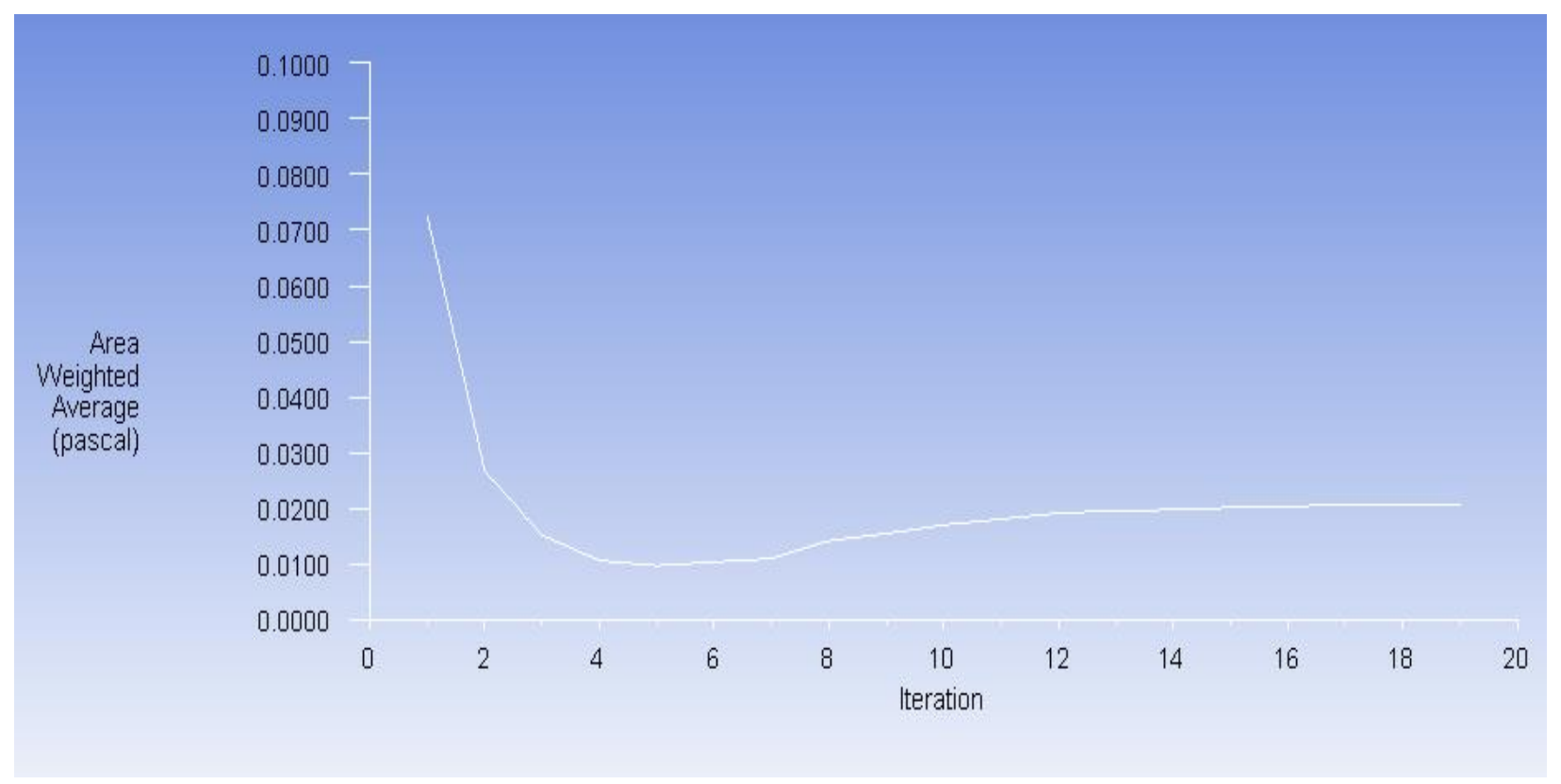

FIGURE 29 - Convergence history of surface shear stress 


\section{RESULTS AND DISCUSSION}

\section{A. General Discussion and Overview}

The versatility of the CFD model allowed for exploration of multiple factors and how they impact shear exposure and nutrient transport within the TPS. The magnitude of shear and advective flow within the TPS had never been characterized prior to this study, so the primary objective of the simulations presented here was to establish the magnitude of shear stresses and flow rates within the scaffold that can be produced under a range of normal operating conditions. Experimental results have already shown increased stimulation of the biosignaling pathways dependent upon shear. Quantifying the magnitude of shear stress in the TPS allows for a better understanding of the magnitudes necessary to activate these pathways. Additionally, prior to this study, it was not known if the media flow within the pores of the scaffold itself provided a significant contribution to overall nutrient transport, or how far beyond the surface of the scaffold media was able to effectively penetrate. By simulating the scaffold particles using the porous media model, the magnitude of the flow within the scaffold material and the relative contributions of advection and diffusion were quantified.

In addition to quantifying the typical range and distribution of shear stress and nutrient transport in the TPS under normal operating conditions, the CFD model was used to study the effects of a number of modifications to the TPS design and operation. Laboratory trials to date have used only alginate as a scaffold material, typically operate at a flow rate of $3 \mathrm{~mL} / \mathrm{min}$, and utilize scaffolds of either $2.23 \mathrm{~mm}$ or $2.75 \mathrm{~mm}$ diameter $(\mathrm{D} / \mathrm{d}=2.848$ or 2.309$)$. The first set of trials presented investigates the effect of packing regime on shear exposure and nutrient transport, using alginate as a scaffold material and simulating media flow at the typical flow rate of 3 
$\mathrm{mL} / \mathrm{min}$. In these trials, all possible packing regimes from $\mathrm{D} / \mathrm{d}=2.00$ to 2.85 are investigated, including the two packing regimes used in experimental trials.

Following the investigation on the influence of packing regime, the two packing regimes used in experimental trials were studied at a higher media flow rate of $10 \mathrm{~mL} / \mathrm{min}$. Fisher et. al. (2011) have studied the effectiveness of the TPS at both flow rates and have found that though higher flow rates show increased expression of osteoblastic markers osteopontin and BMP-2, mineralization is diminished at the higher flow rate. This is hypothesized to be the result of too much flow through the scaffold, resulting in dissolution of the cell-scaffold complex. This hypothesis was tested using the CFD model by comparing the results between the two flow rates.

The third set of trials presented compares the effectiveness of "maximal contact" crystal structures, to less ideal "line slip" crystal structures. Within a given packing regime, the "maximal contact" structure corresponds to the $\mathrm{D} / \mathrm{d}$ value at which volume fraction is maximized. The contact patterns in these structures form perfect helices along the axial direction of the cylindrical growth chamber. "Line slip" structures, by contrast, have less-than-ideal D/d ratios for the given packing regime, resulting in a "staggered helix" with greater void space. Prior to this study, it was hypothesized that maximal contact structures would promote greater shear exposure due to their lower void fraction. This hypothesis was tested by comparing three "line slip" structures to their corresponding "maximal contact" structures.

The final set of trials presented studies the effects of using a more permeable scaffold material in place of alginate. Tricalcium phosphate is a common ceramic material used in bone tissue engineering due to its mechanical strength and osteoconductive properties. The design of the TPS is such that a number of different biomaterials would be a good candidate for scaffold materials. Tricalcium phosphate was simulated to determine the degree to which advective flow is 
enhanced when using a much more permeable material, and to determine whether or not diffusive transport still dominates.

For greater clarity and for reference, each of the packing regimes simulated is represented below. The special cases in which the unit cells are defined by flat planes perpendicular to the cylinder axis are denoted C2, C3, C4, and C5, consistent with Mughal et. al. Maximal contact structures are denoted MC1, MC2, MC3, MC4, MC5, MC6, and MC7, while line slip structures are denoted LS1, LS2, and LS3. These structures were validated both by comparing the measured void fraction to the expected value and by visual comparison to the structures characterized by Mughal. In some cases, a disparity was observed between expected void fraction and observed void fraction. However, this is likely due to the inherent difficulty in measuring void fraction. Because all trials were normalized to a column height of $30 \mathrm{~mm}$, some of the simulated geometries used in this study contain partial unit cells. In this scenario, average void fraction over the column may not be representative of the true void fraction, and a visual inspection of the geometry is a better indicator of whether or not the crystal structure conforms to that predicted by Mughal. The following figures show each simulated packing regime, with figures from Mughal for comparison. In each case, the contact patterns were carefully matched and stacking faults were avoided by piecewise addition of the beads. 
TABLE I - SUMMARY OF D/d VALUES FOR ALL PACKING REGIMES TESTED

\begin{tabular}{|c|c|c|}
\hline Packing Regime & $\mathbf{D} / \mathbf{d}$ & $\mathbf{d}(\mathbf{m m})$ \\
\hline C2 & 2.0000 & 3.1750 \\
\hline MC1 & 2.0390 & 3.1143 \\
\hline C3 & 2.1547 & 2.9470 \\
\hline MC2 & 2.2247 & 2.8543 \\
\hline MC3 & 2.2905 & 2.7723 \\
\hline LS1 & 2.3800 & 2.6681 \\
\hline C4 & 2.4142 & 2.6303 \\
\hline MC4 & 2.4863 & 2.5540 \\
\hline LS2 & 2.5440 & 2.4961 \\
\hline MC5 & 2.5712 & 2.4697 \\
\hline LS3 & 2.6550 & 2.3917 \\
\hline C5 & 2.7013 & 2.3507 \\
\hline MC6 & 2.7306 & 2.3255 \\
\hline MC7 & 2.8211 & 2.2509 \\
\hline
\end{tabular}

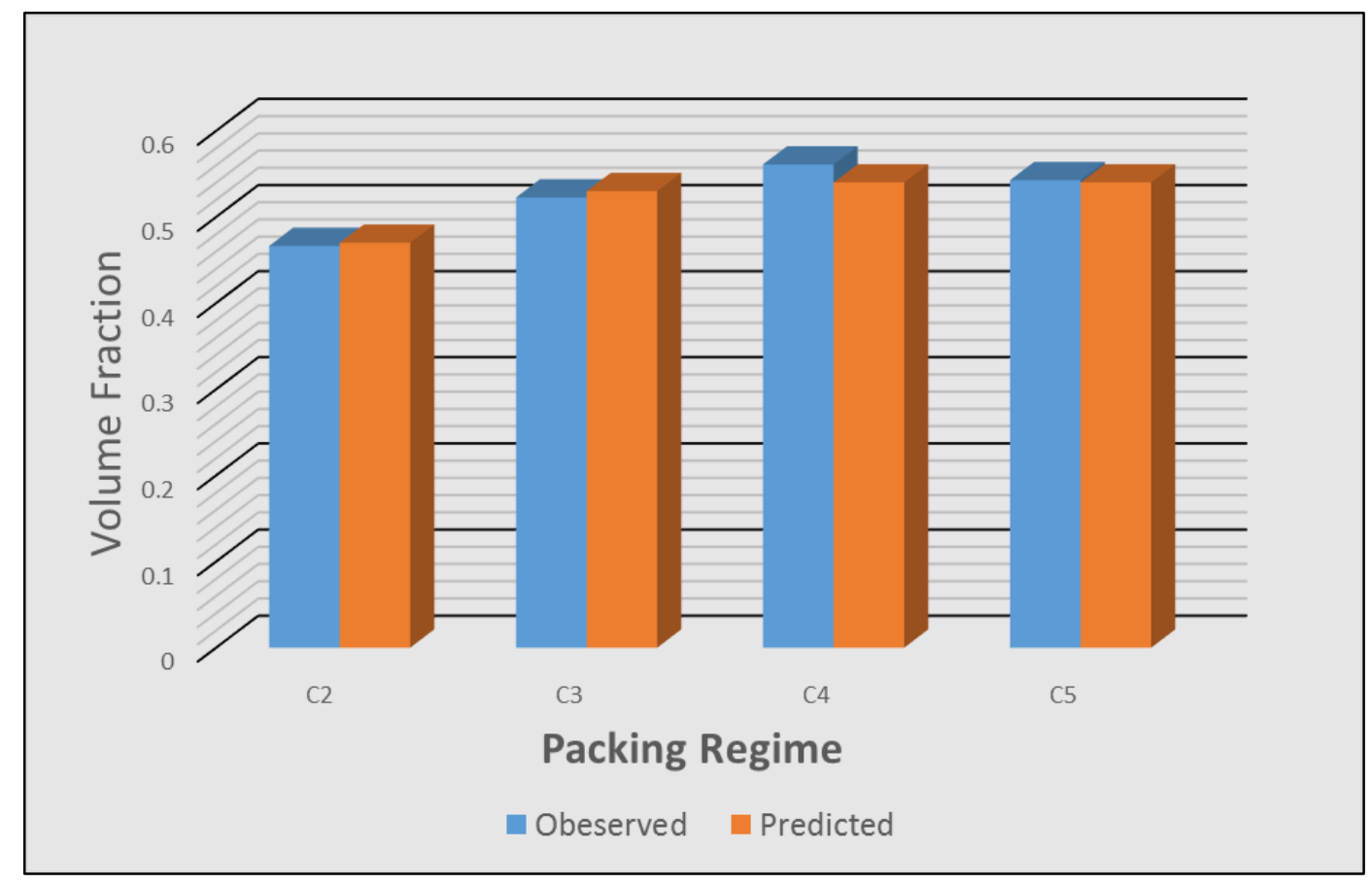

FIGURE 30 - Observed volume fraction for C2 - C5 with Mughal predicted values 

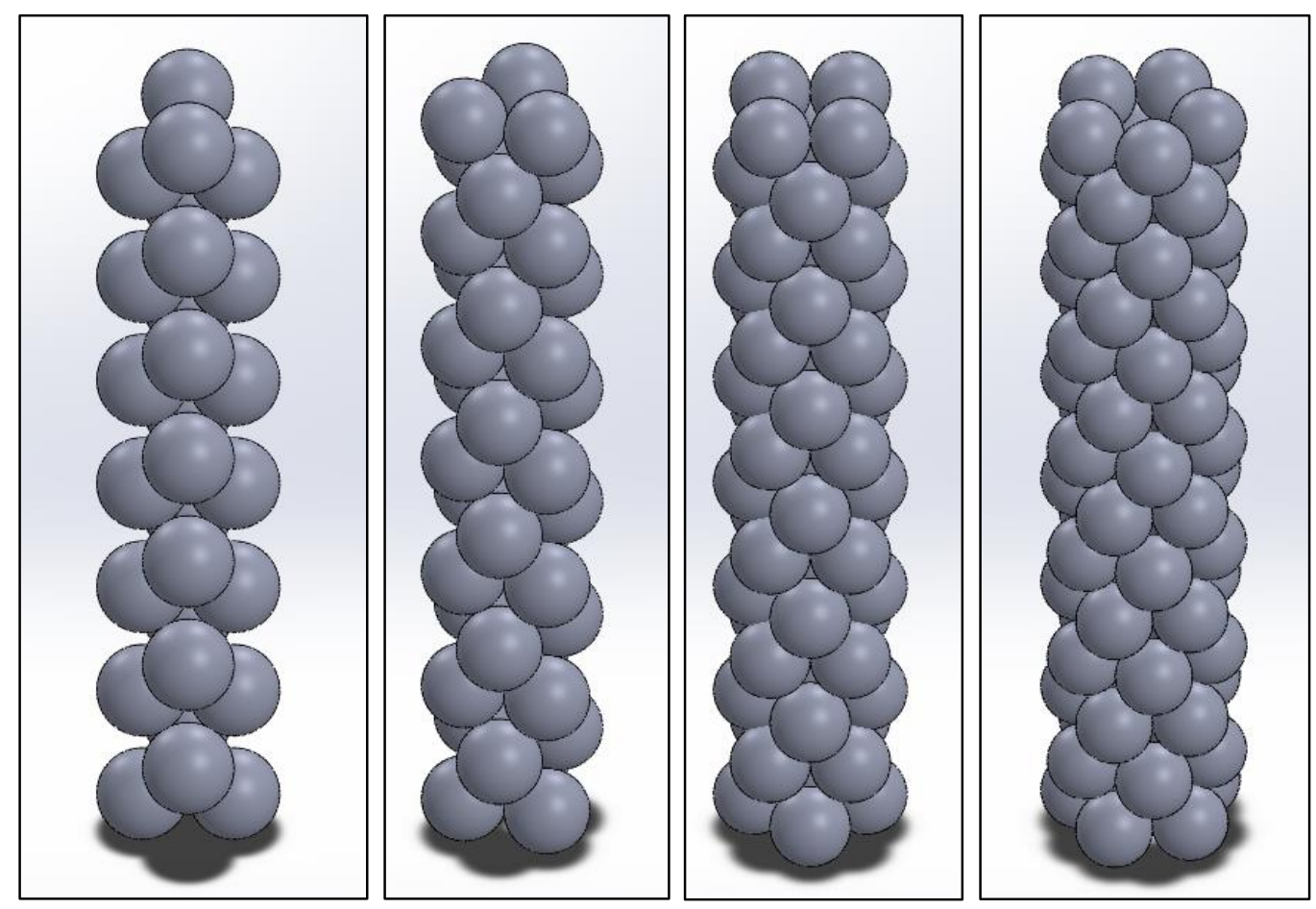

FIGURE $31-\mathrm{C} 2, \mathrm{C} 3, \mathrm{C} 4$, and $\mathrm{C} 5$ isometric views

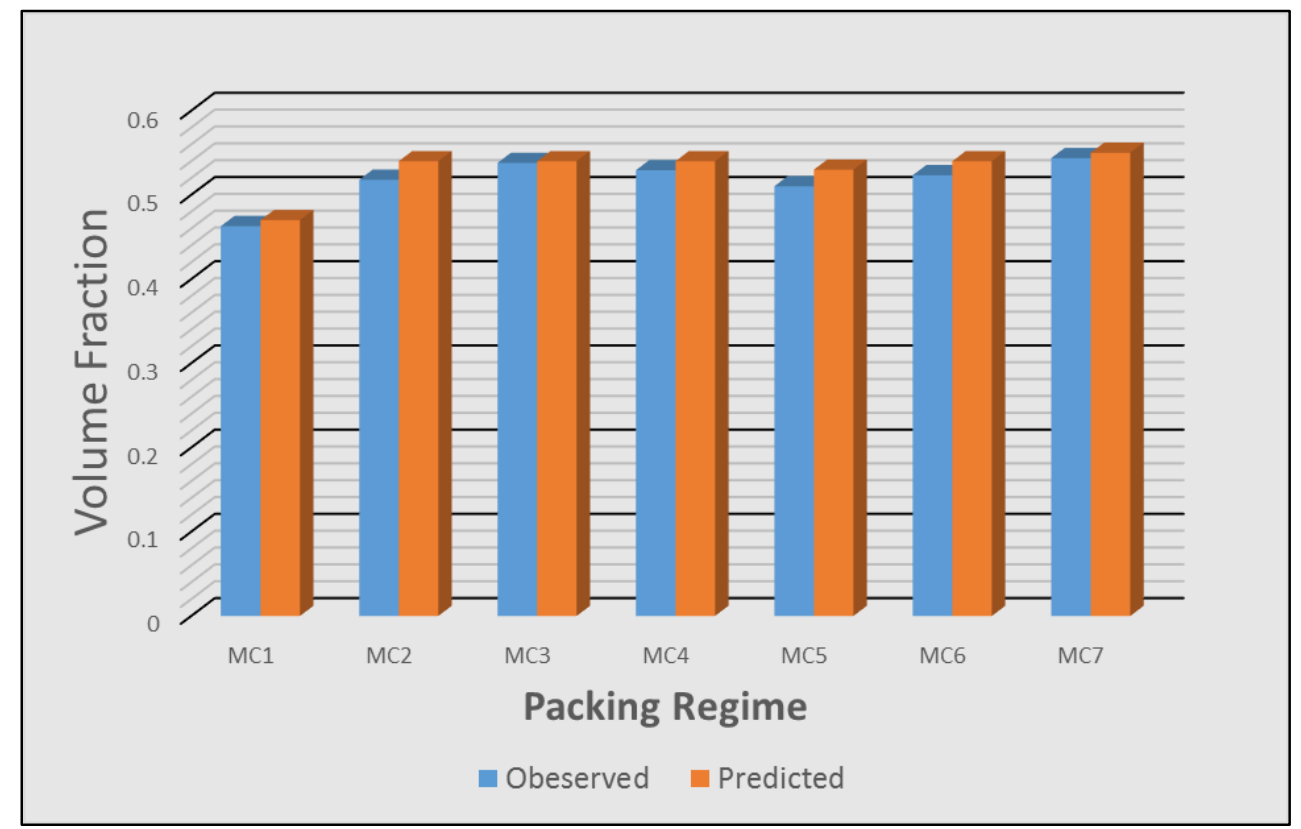

FIGURE 32 - Observed volume fraction for MC1-MC7 with Mughal predicted values 

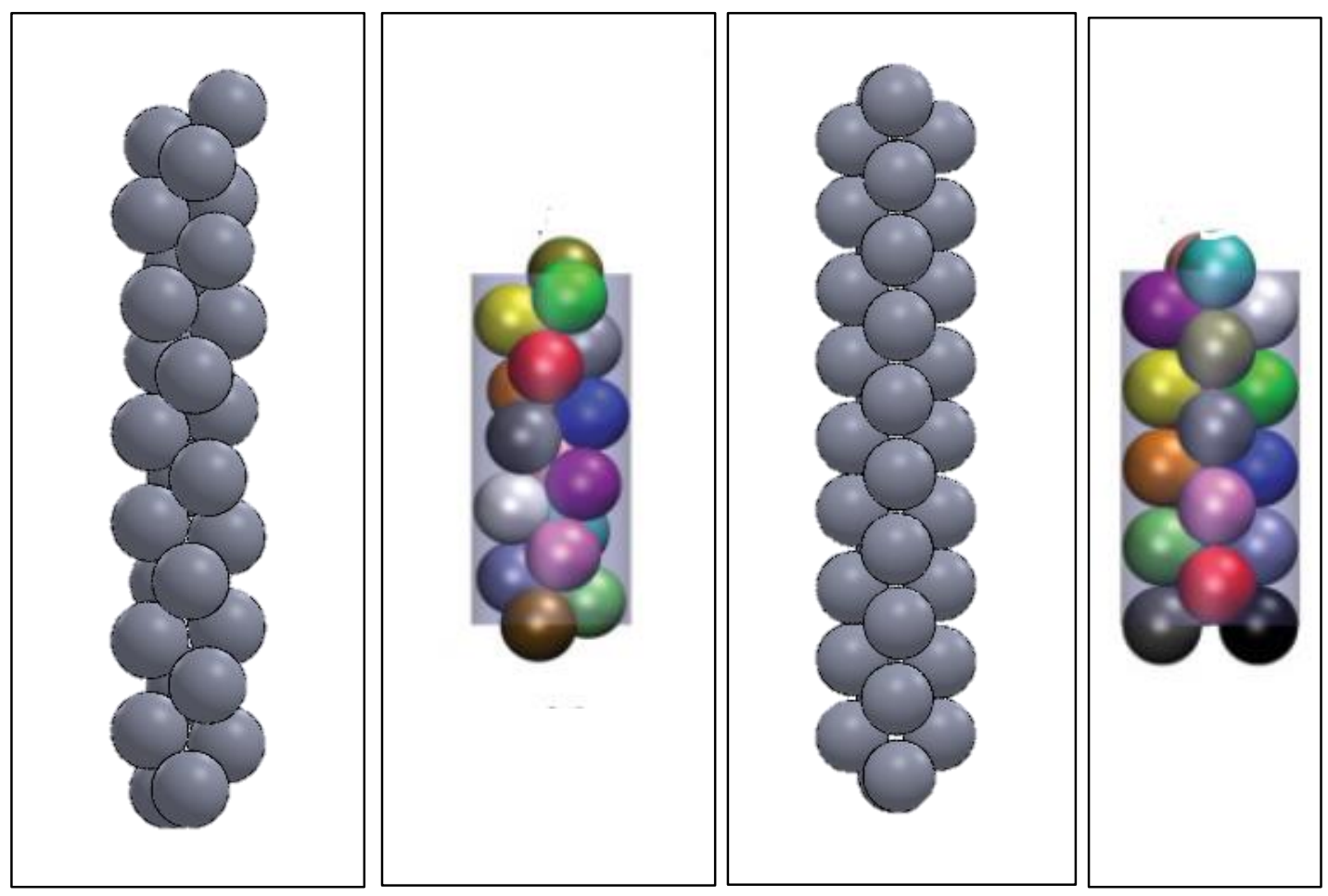

FIGURE 33 - MC1 and MC2 (grey) compared with Mughal models generated by Monte Carlo
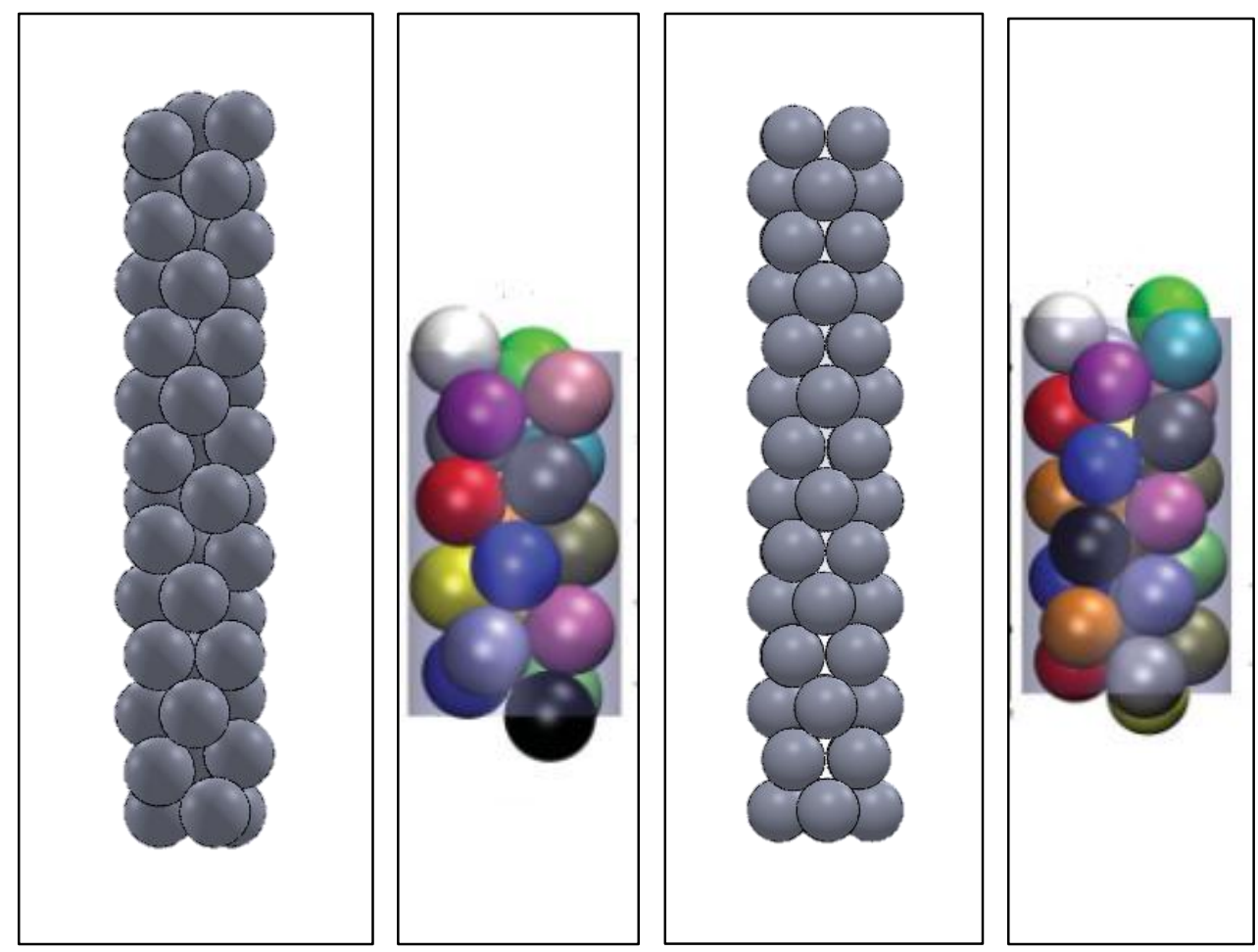

FIGURE 34 - MC3 and MC4 (grey) compared with Mughal models generated by Monte Carlo 

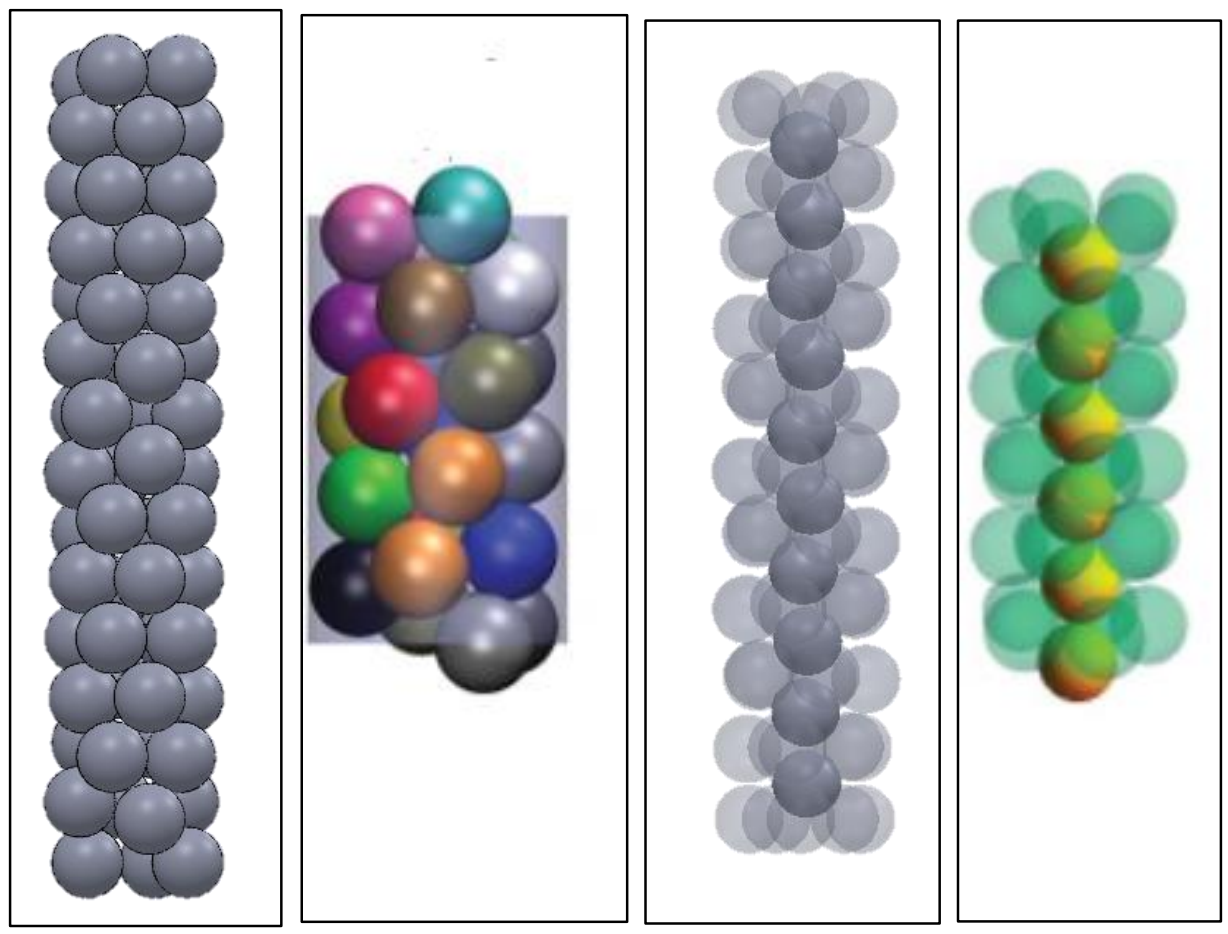

FIGURE 35 - MC5 and MC6 (grey) compared with Mughal models

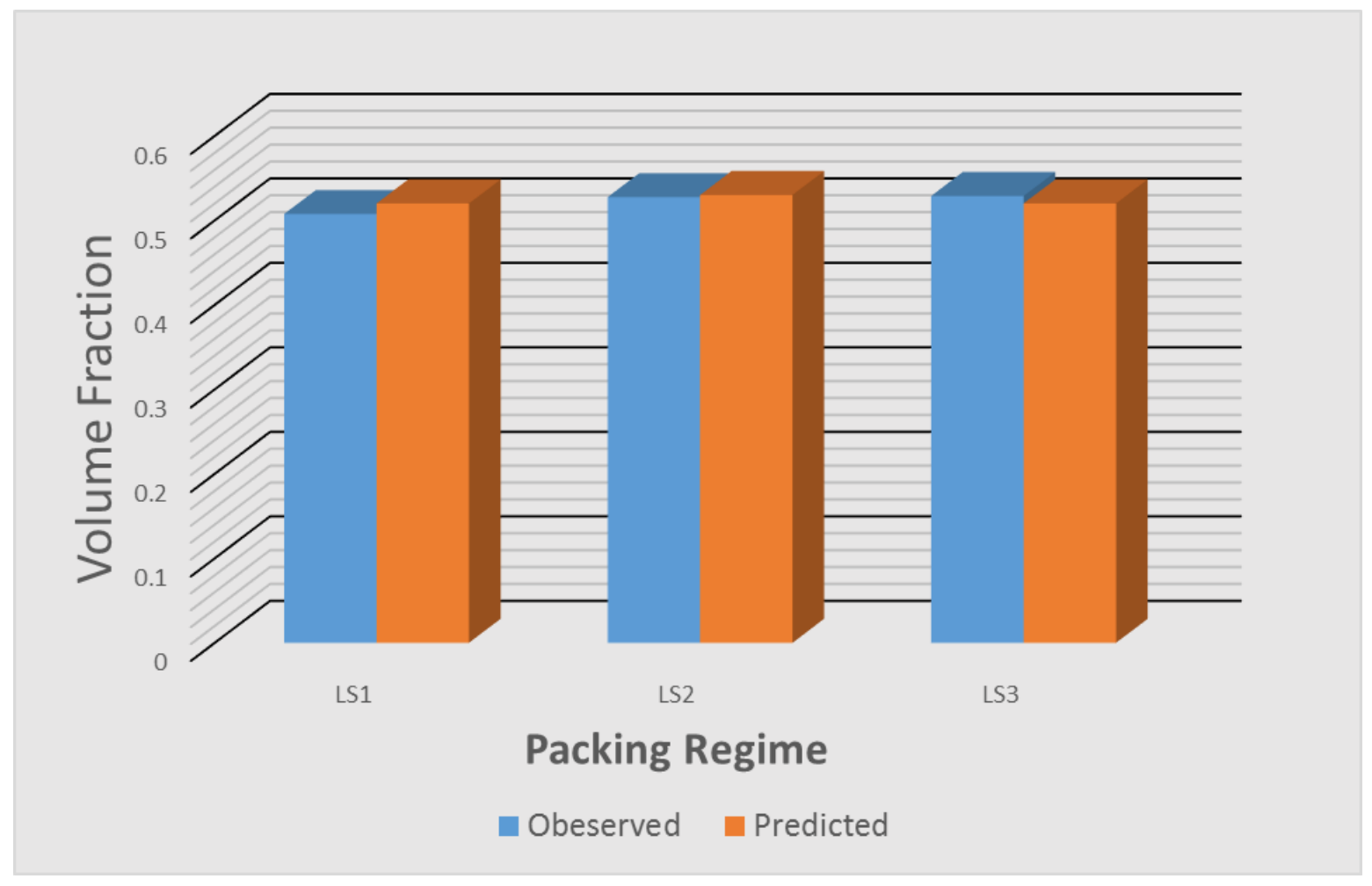

FIGURE 36 - Observed volume fraction for LS1-LS3 with Mughal predicted values 

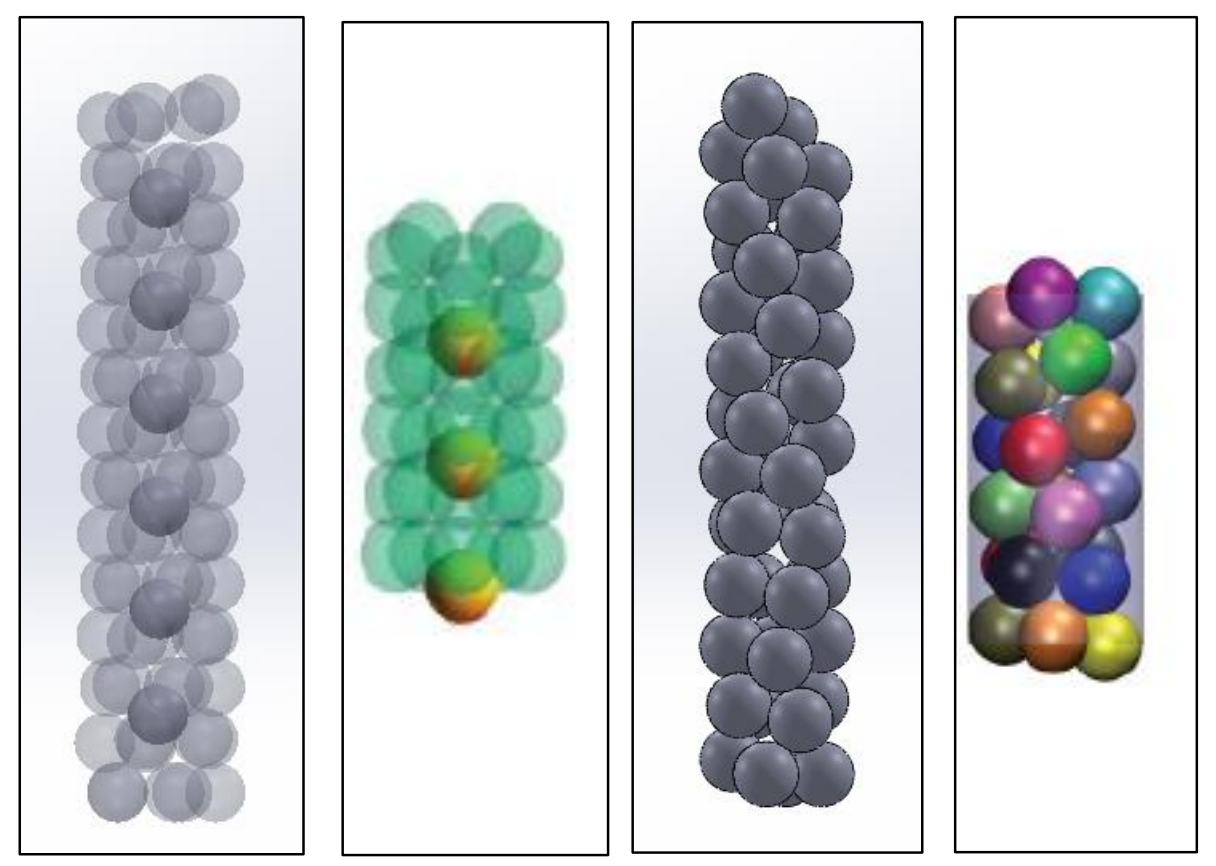

FIGURE 37 - MC7 and LS1 (grey) compared with Mughal models
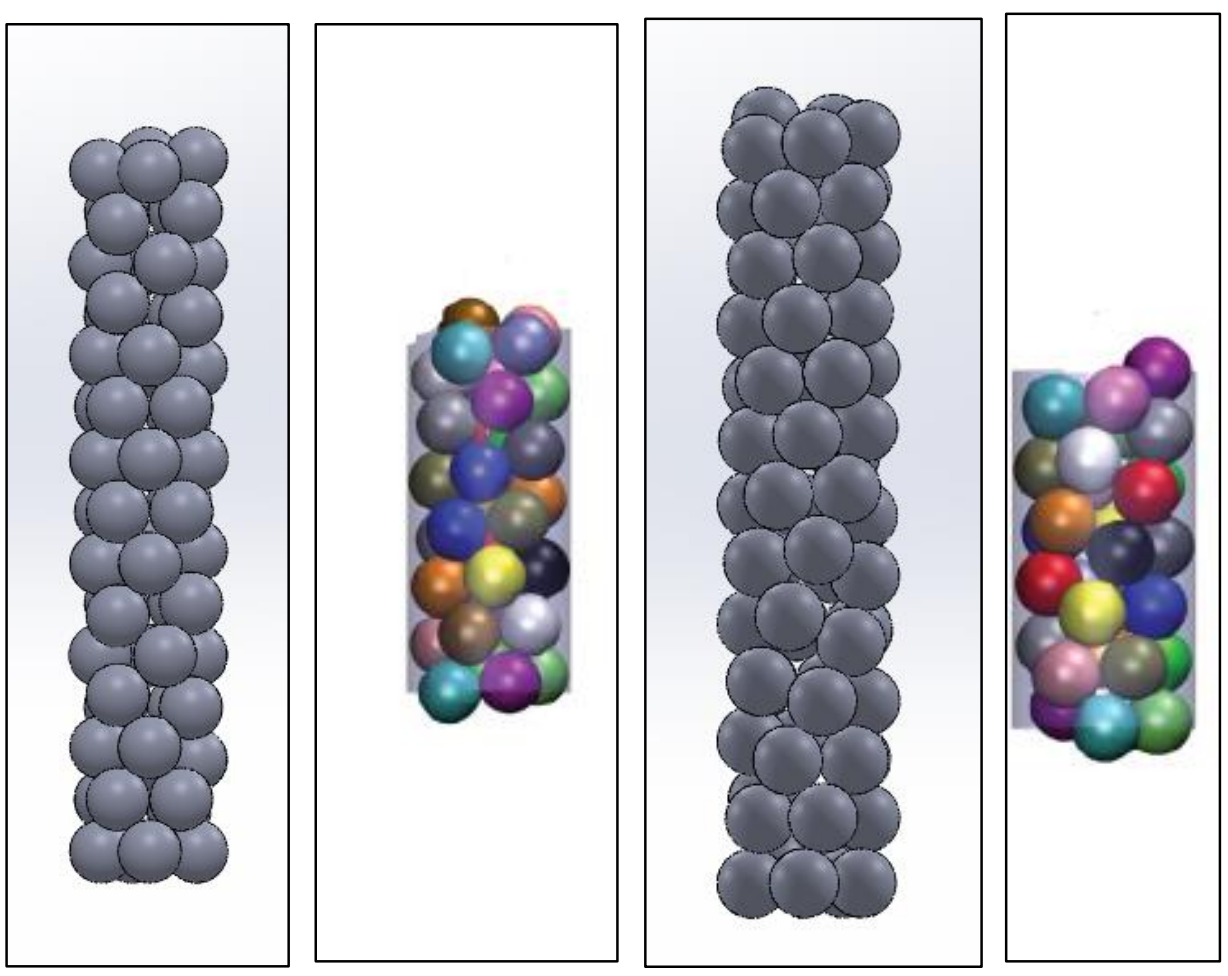

FIGURE 38 - LS2 and LS3 compared with Mughal models 


\section{B. Shear Stress and Packing Regime}

In this section results are presented for shear distribution along the scaffold surface of each maximal contact structure, representing each possible packing regime. Each of these trials was conducted at a flow rate of $3 \mathrm{~mL} / \mathrm{min}$. For each individual case, shear data is presented both qualitatively and quantitatively. Histograms for each case illustrate the distribution of shear stress magnitudes over the surface of all beads, with the y axis representing \% surface coverage for each range of values. Additionally, contour maps allow for the visualization of where shearing forces are concentrated on the bead surfaces. Prior to individual analysis of each case, a composite average of shear stress magnitude over the surface of all beads was calculated for each case and compiled in FIGURE 36. The packing regimes tested are ordered along the $\mathrm{x}$-axis as D/d increases (or, alternatively, as bead diameter becomes smaller).

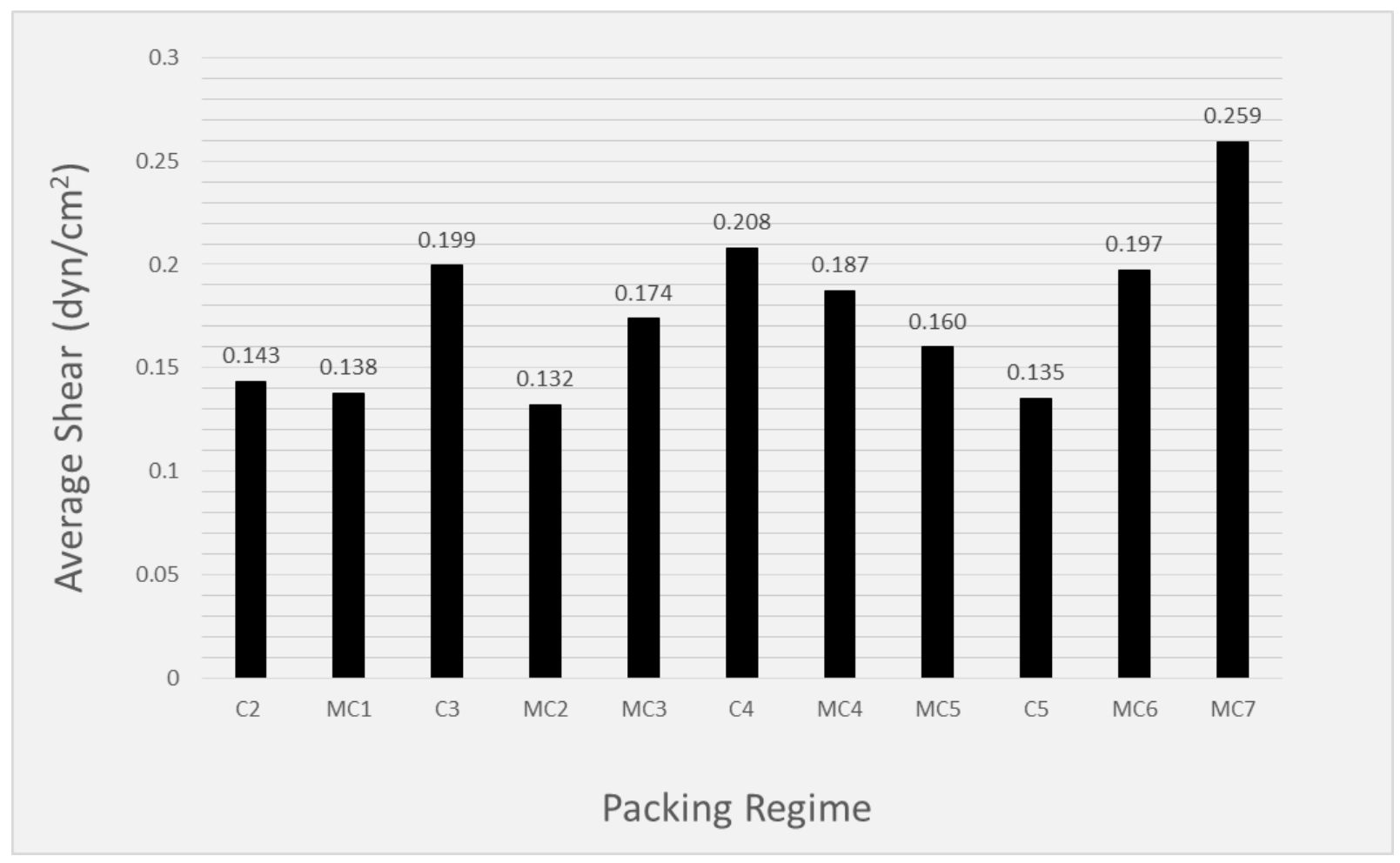

FIGURE 39 - Composite shear average for all maximal contact structures 
The data presented in FIGURE 39 suggests that the average shear stress for all packing regimes tested is on the order of 0.13 to $0.26 \mathrm{dyn} / \mathrm{cm}^{2}$ at a flow rate of $3 \mathrm{~mL} / \mathrm{min}$. This is significantly lower than that predicted by the 2D COMSOL model. This is to be expected as the COMSOL model included several limiting assumptions. In order to more accurately replicate the geometry in two dimensions, a $4 \mathrm{~mm}$ diameter $(\mathrm{D} / \mathrm{d}=1.5)$ bead was modeled, which is outside the scope of typical operation of the TPS. Additionally, a 2-dimensional model cannot accurately replicate the proportion of the bead surface area exposed to shearing flow, and the 2D model also allowed space between the chamber wall and beads. These factors combined would certainly raise the average shear stress above its true value. FIGURE 40 shows the velocity field generated using the COMSOL model developed by Fisher et. al.

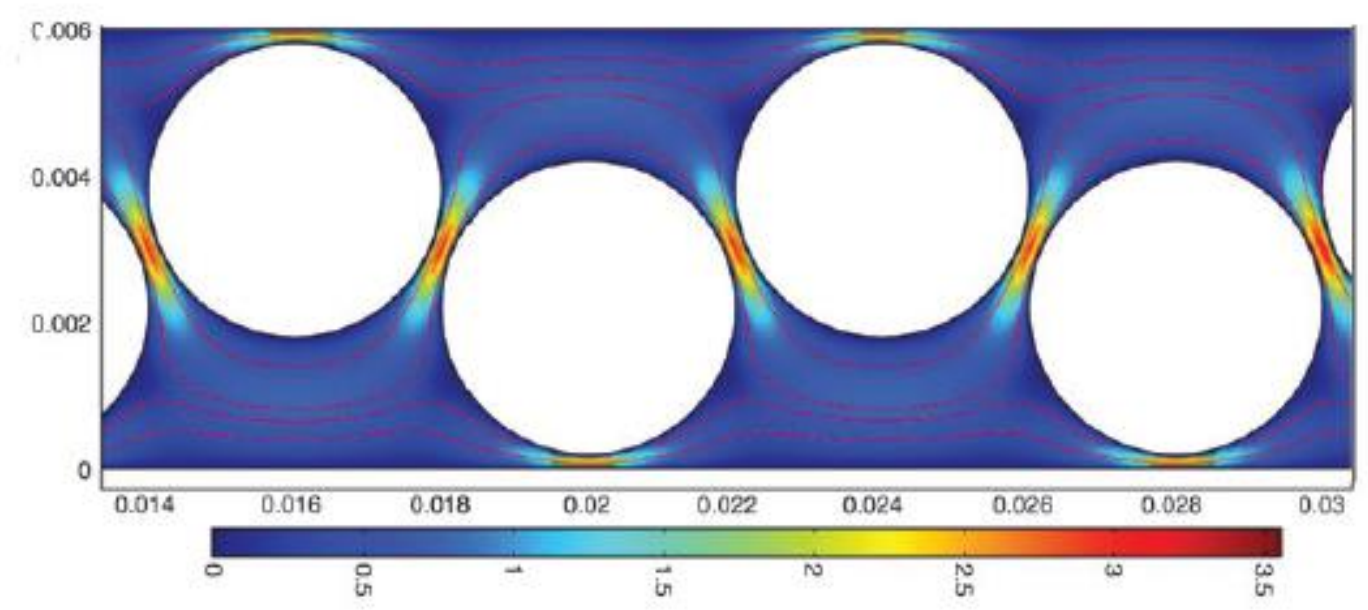

FIGURE 40 - Velocity plot from 2D COMSOL model reproduced from Fisher et. al. (2011)

Though the shear stress magnitudes observed using the 3D CFD model are less than those predicted in the COMSOL study, all still fall within the range determined to enhance osteopontin and BMP-2 expression. As mentioned previously, Li et. al. demonstrated that shear stress values of only $0.15 \mathrm{dyn} / \mathrm{cm}^{2}$ were sufficient to enhance osteoblastic marker expression and mineralization. 
This knowledge provides some validation for the model, as the average shear stresses observed are congruent with the experimental findings by Fisher showing enhanced differentiation.

Another important general observation is the difference in flow field between geometries containing inner spheres and those that do not. As predicted, the velocity fields indicate that a majority of the media flow is directed through the central annulus in packing regimes where inner spheres are not present. This isolates the majority of high shear and nutrient transport to the region of the beads in contact with the central annulus, leaving little media flow in the outer regions of the growth chamber. Thus, shear coverage was found to be more uniform in those geometries containing interior spheres. This finding is important to understanding the relationship between packing regime and bioreactor function, and is revisited a number of times in the following sections.
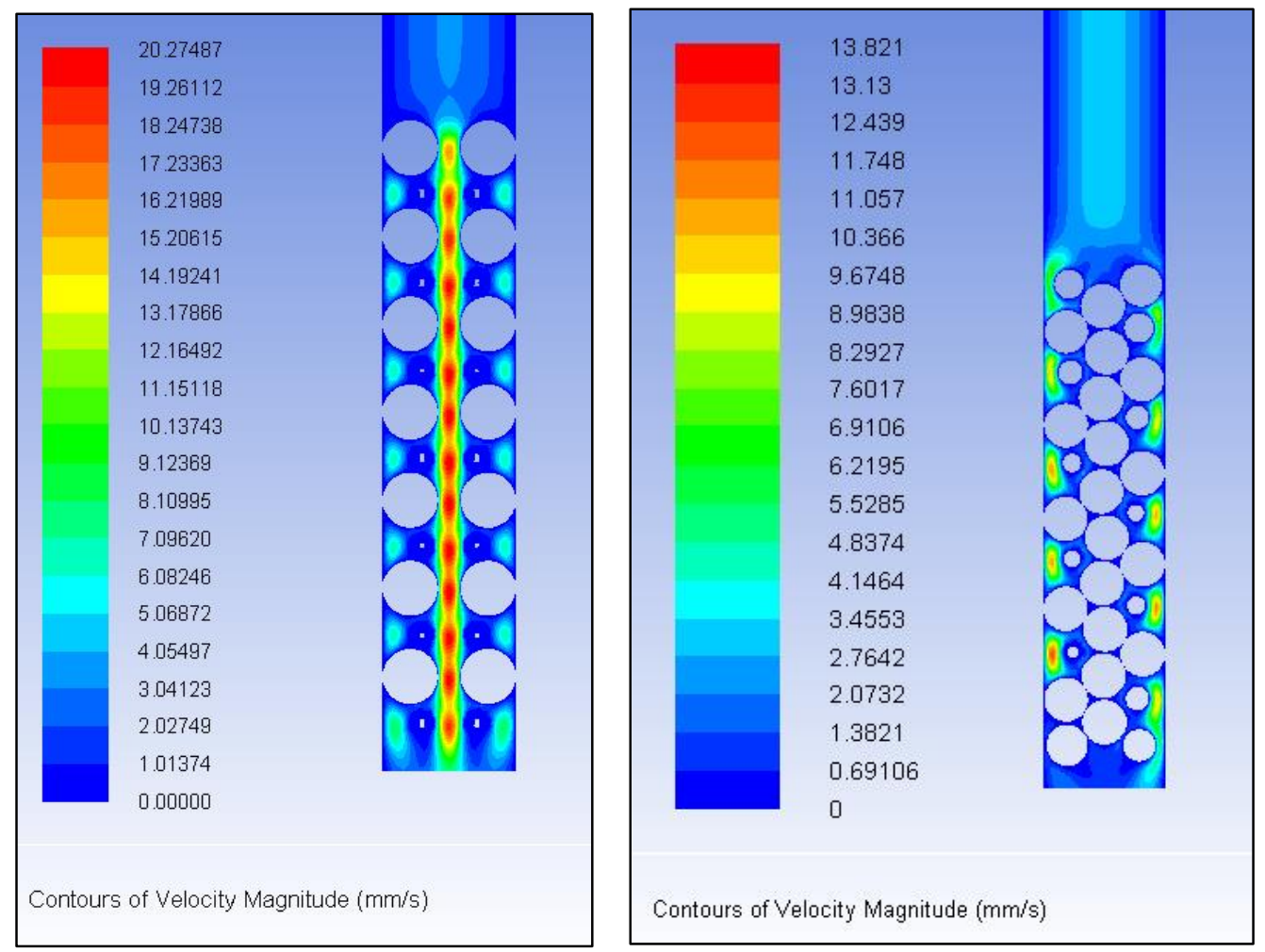

FIGURE 41 - Comparison of velocity field without (left) and with (right) interior spheres 
What follows is a characterization of each packing regime with a histogram and contour plot illustrating the shear stress distribution unique to each geometry. The $\mathrm{x}$-axis is normalized to a maximum value of $0.75 \mathrm{dyn} / \mathrm{cm}^{2}$ in all plots.

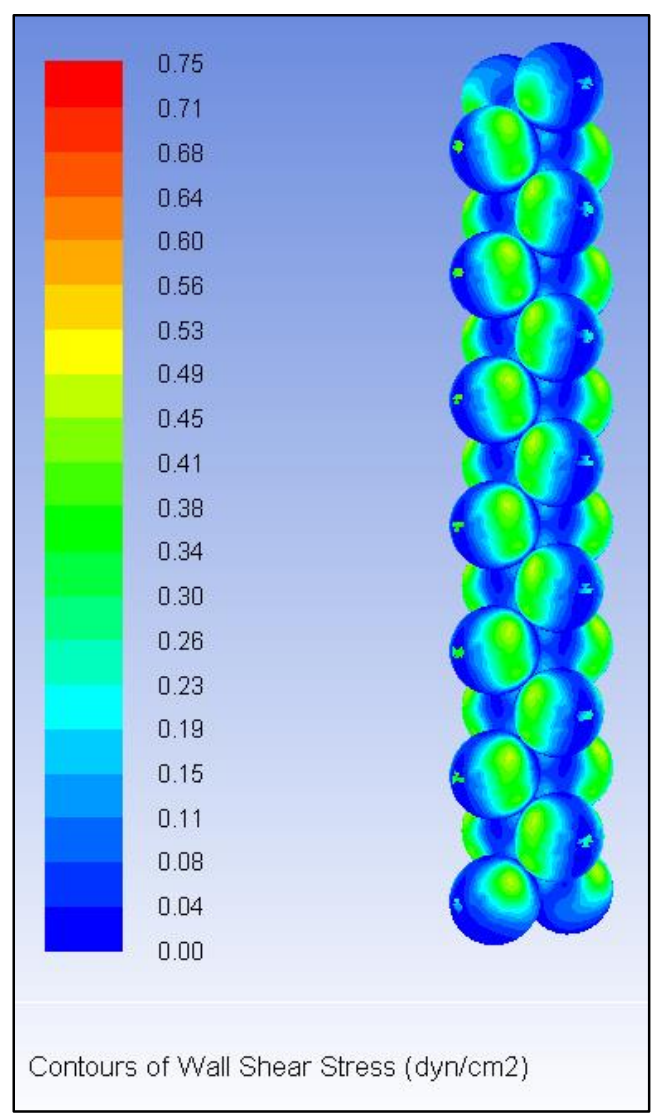

FIGURE 42 - Contour map of shear distribution on C2 $(\mathrm{D} / \mathrm{d}=2.00)$ 


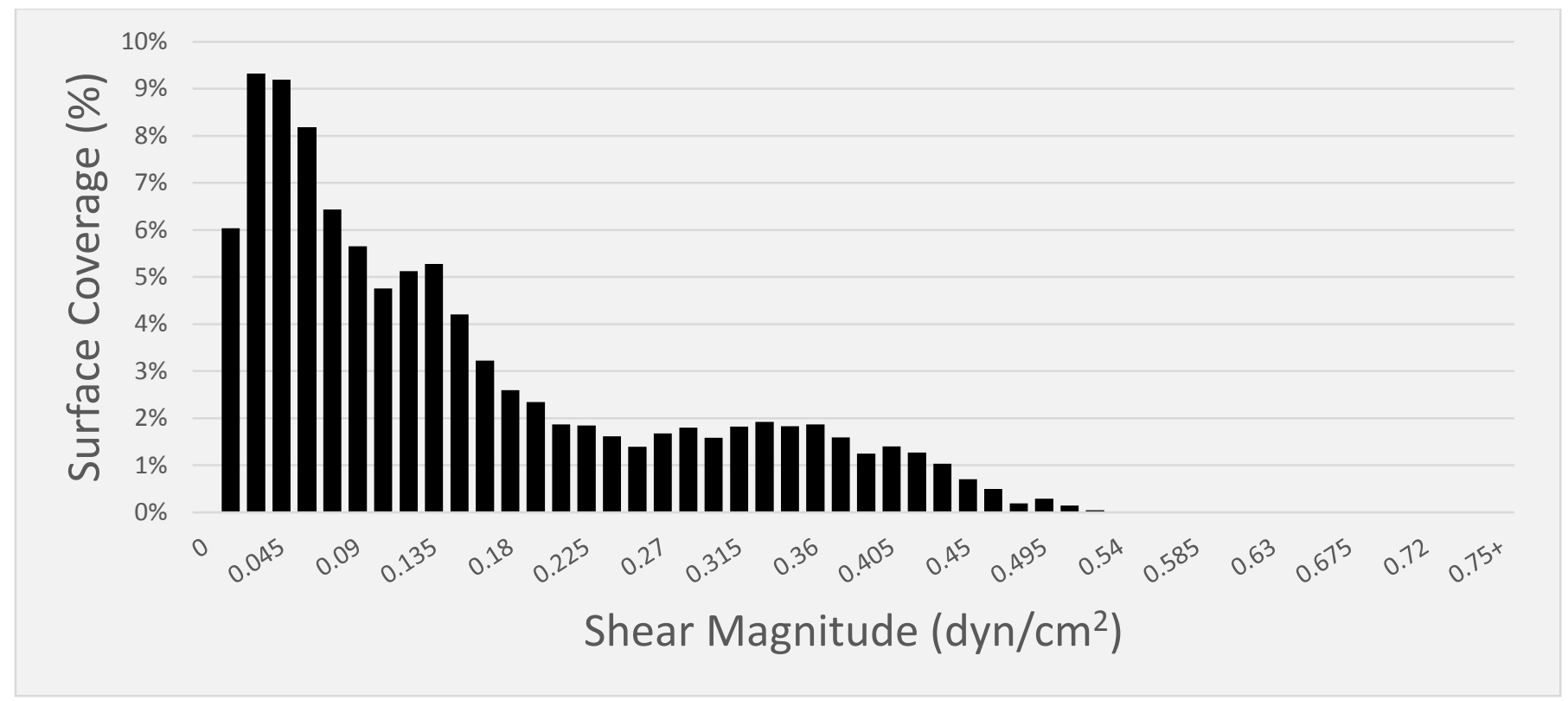

FIGURE 43 - Histogram of shear magnitudes for $\mathrm{C} 2(\mathrm{D} / \mathrm{d}=2.00)$

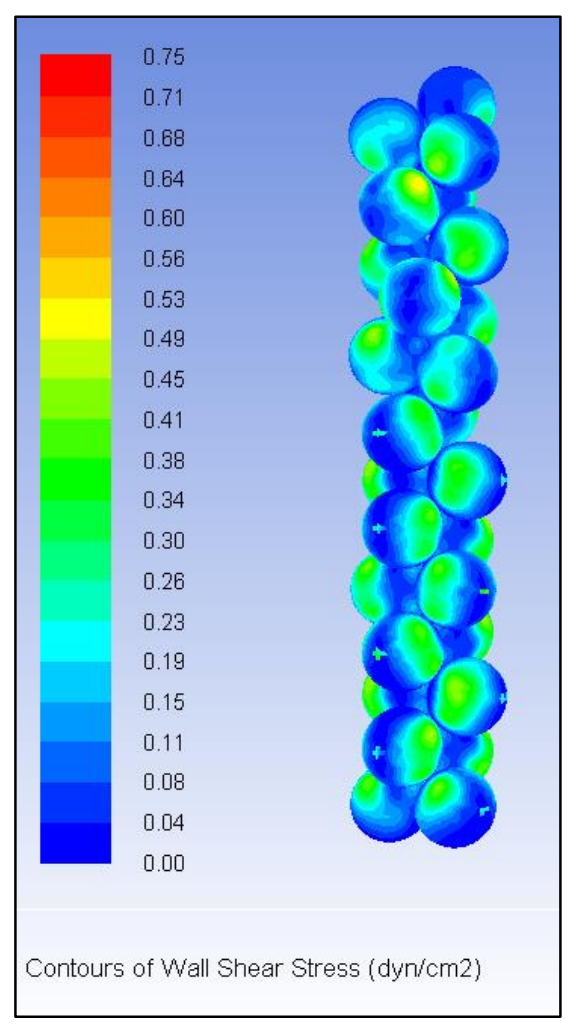

FIGURE 44 - Contour map of shear distribution on MC1 $(\mathrm{D} / \mathrm{d}=2.039)$ 


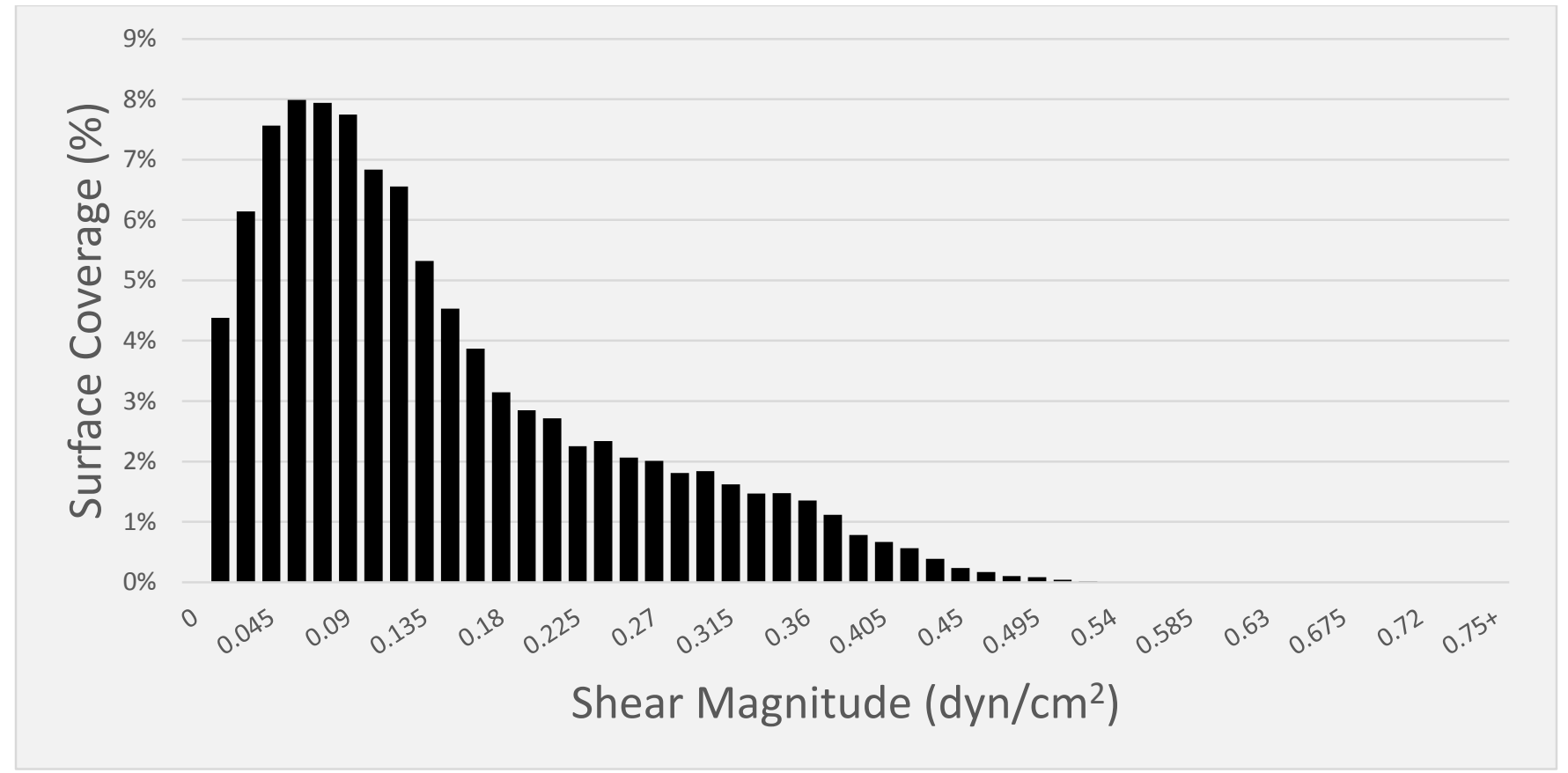

FIGURE 45 - Histogram of shear magnitudes for MC1 (D/d = 2.039)

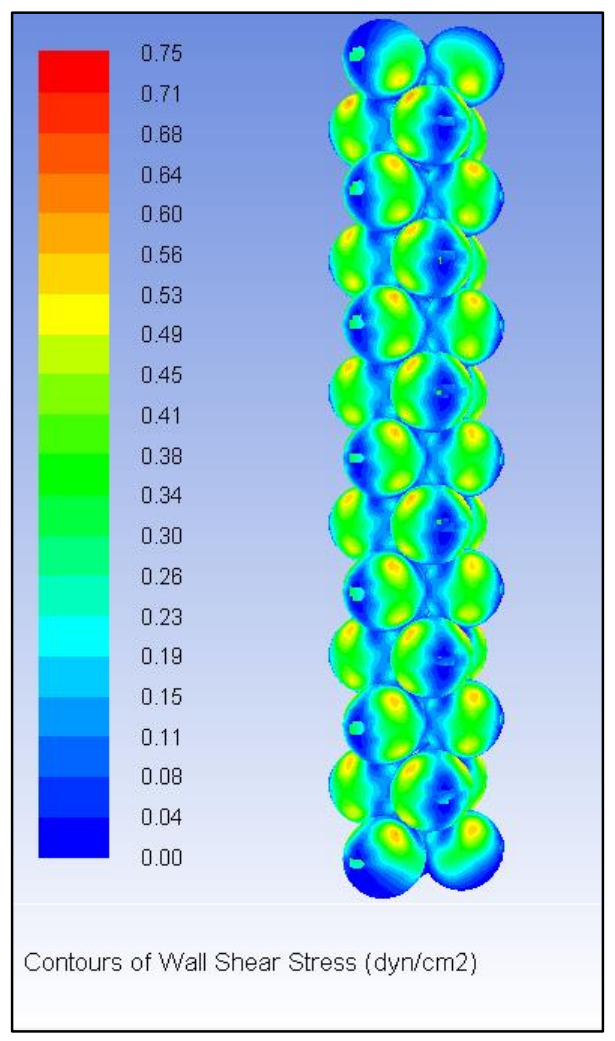

FIGURE 46 - Contour map of shear distribution on C3 $(\mathrm{D} / \mathrm{d}=2.155)$ 


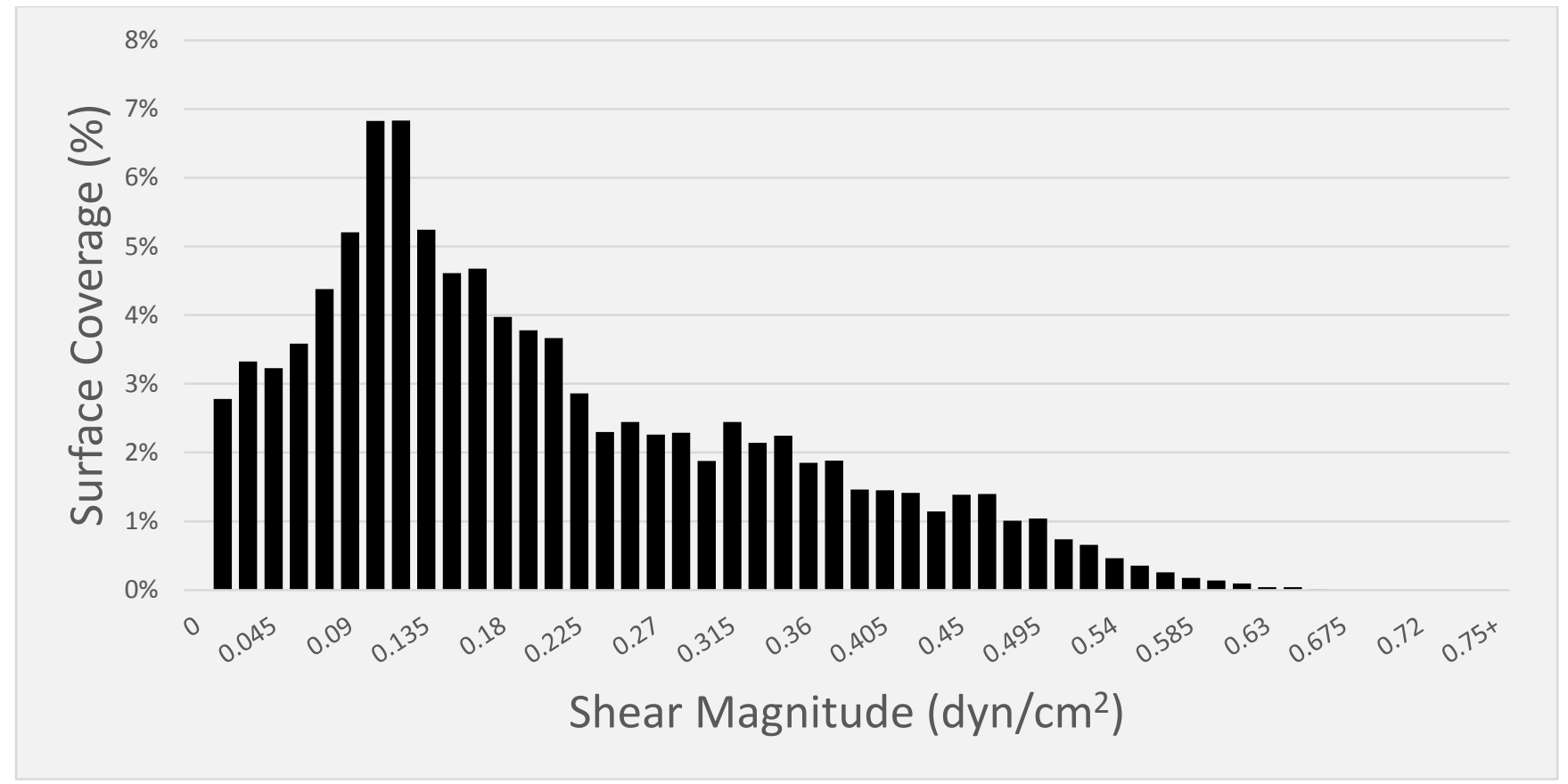

FIGURE 47- Histogram of shear magnitudes for C3 $(\mathrm{D} / \mathrm{d}=2.155)$
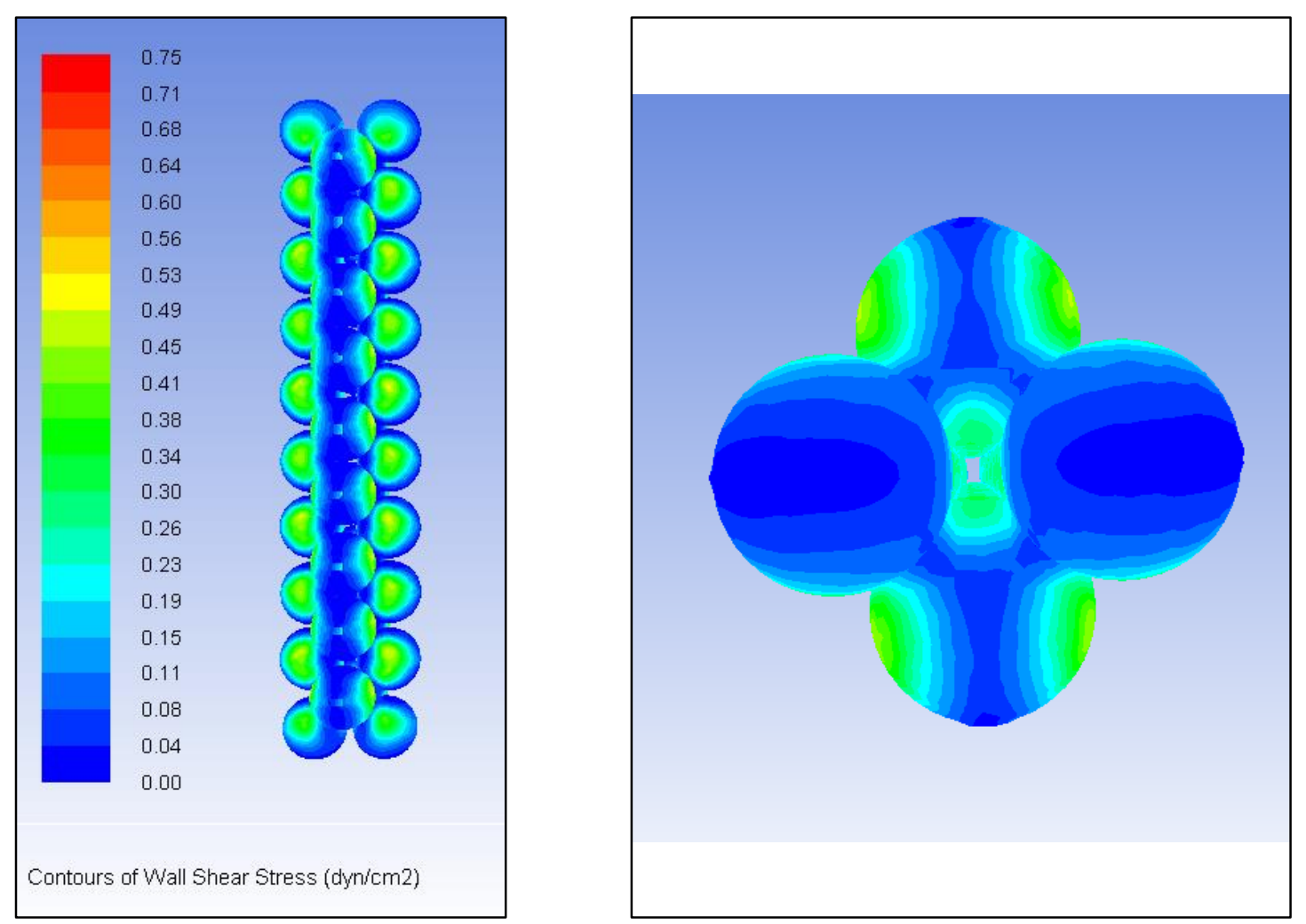

FIGURE 48 - Contour map of shear distribution on MC2 (D/d = 2.225) 


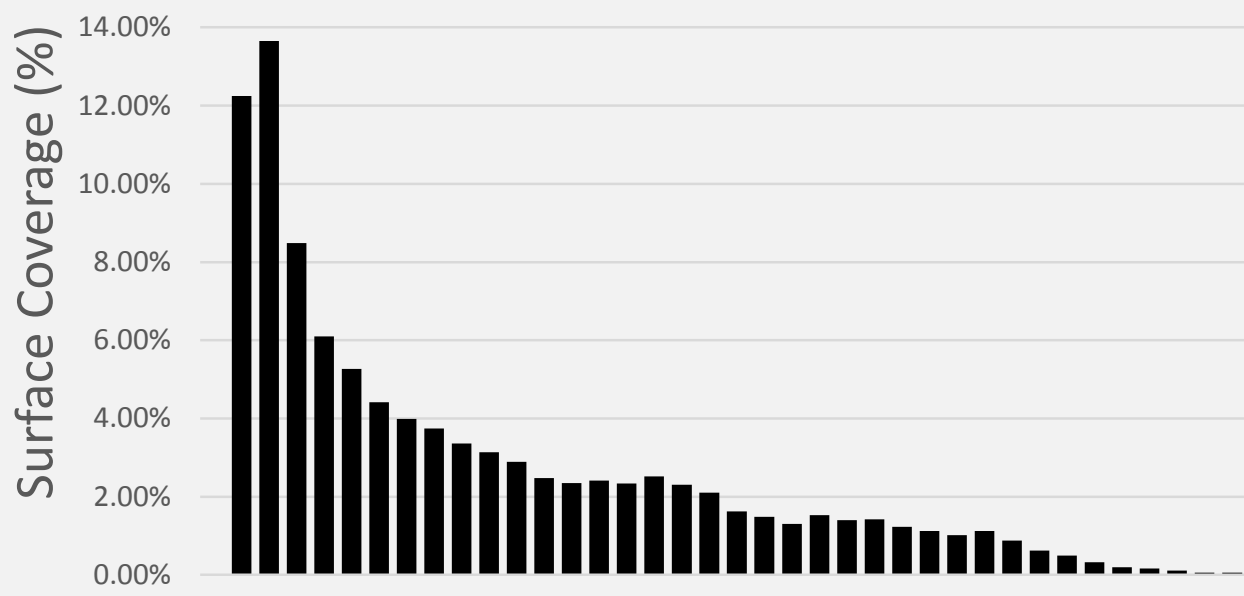

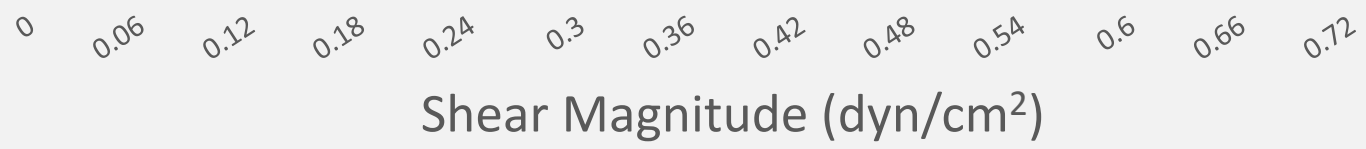

FIGURE 49 - Histogram of shear magnitudes for MC2 (D/d = 2.225)
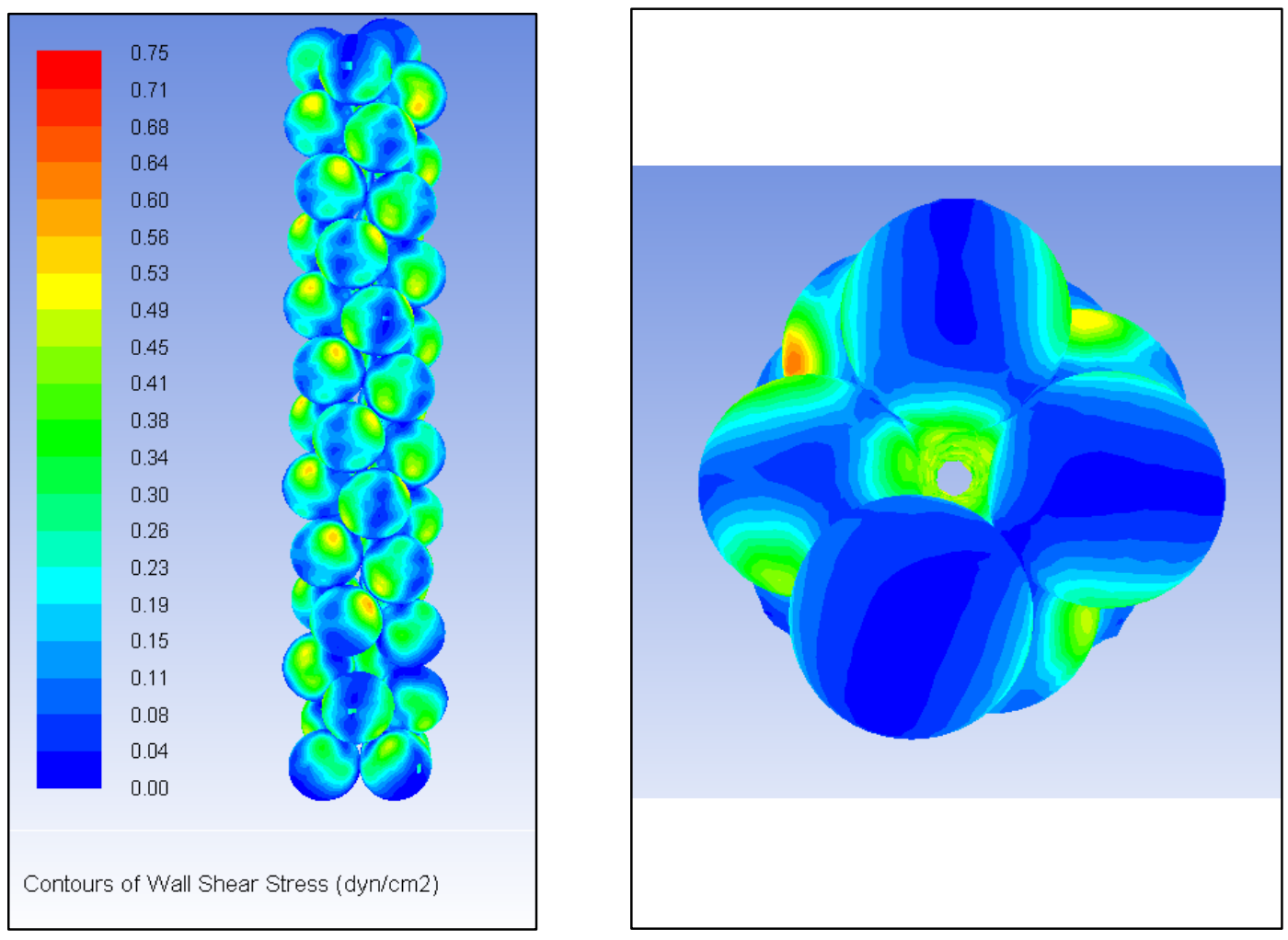

FIGURE 50 - Contour map of shear distribution on MC3 (D/d = 2.291) 


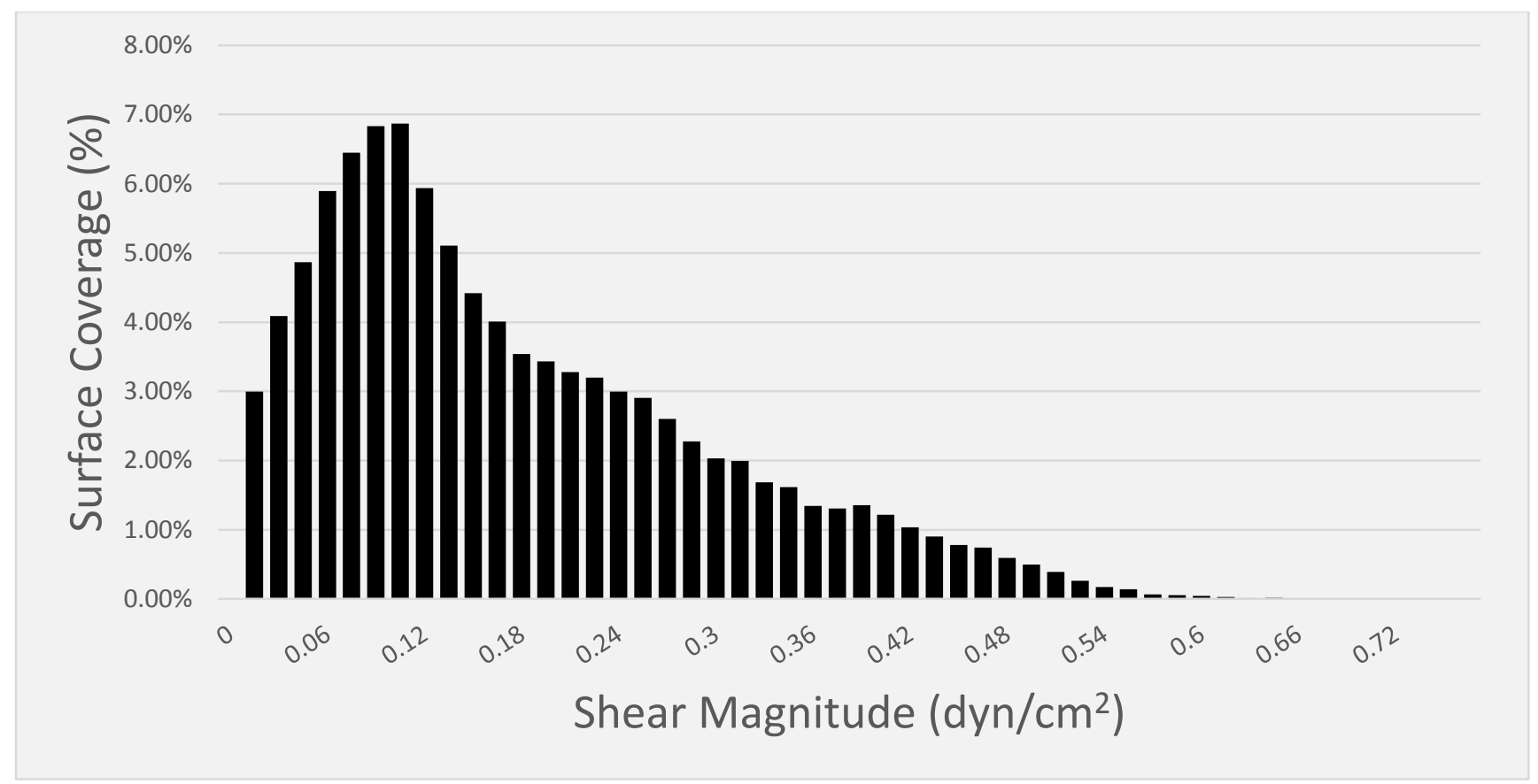

FIGURE 51 - Histogram of shear magnitudes for MC3 (D/d = 2.291)
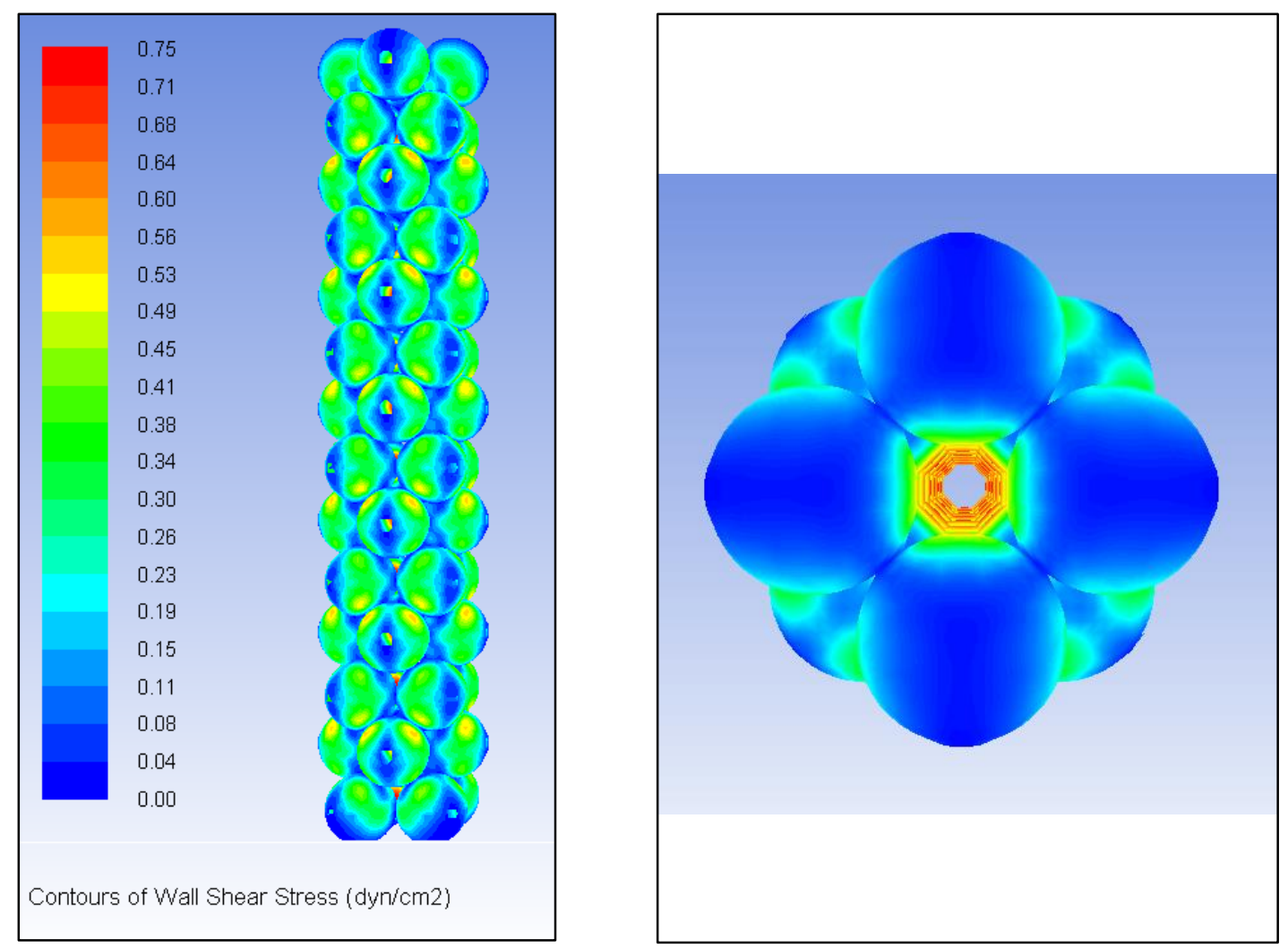

FIGURE 52 - Contour map of shear distribution on C4 $(\mathrm{D} / \mathrm{d}=2.414)$ 


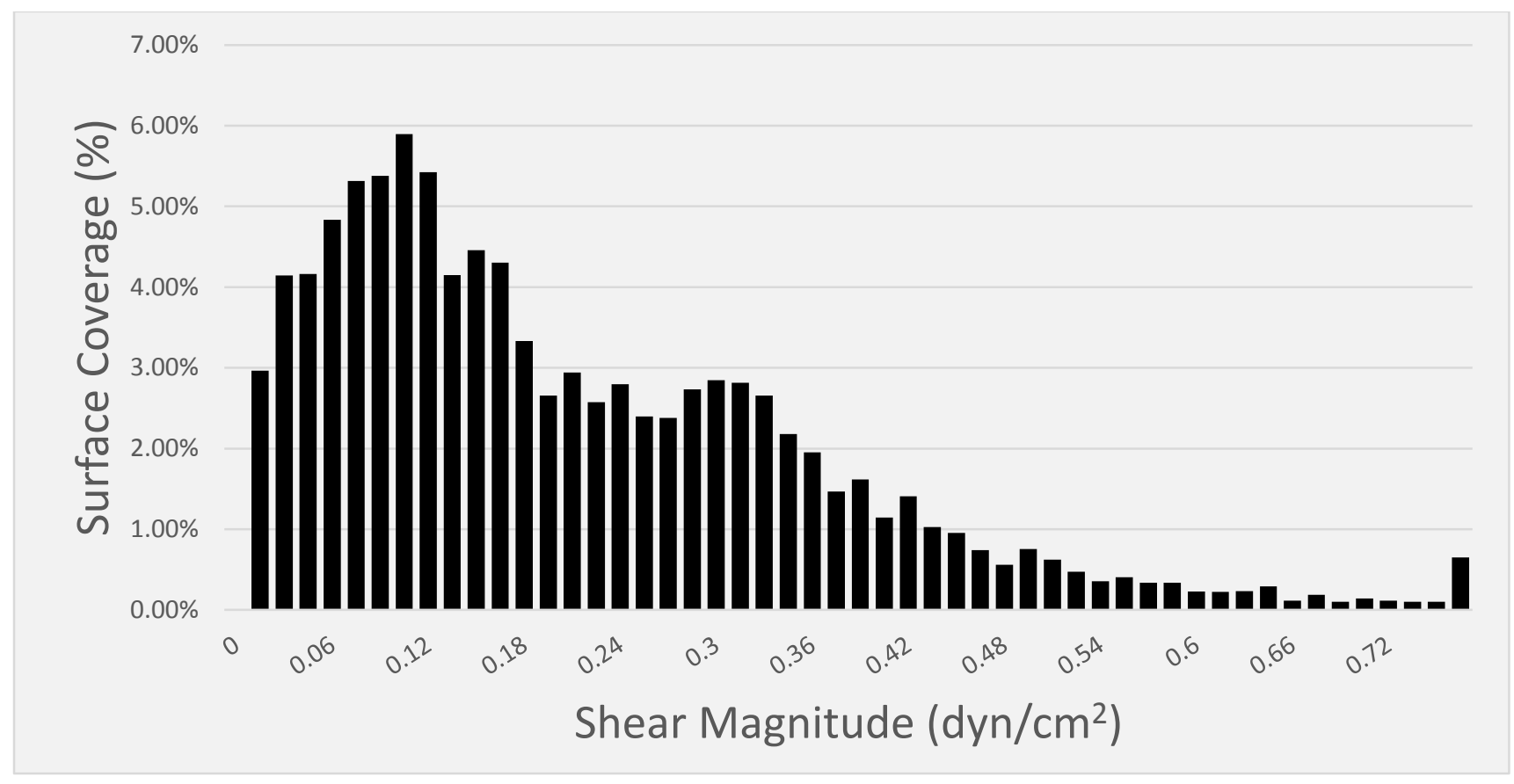

FIGURE 53 - Histogram of shear magnitudes for C4 $(\mathrm{D} / \mathrm{d}=2.414)$
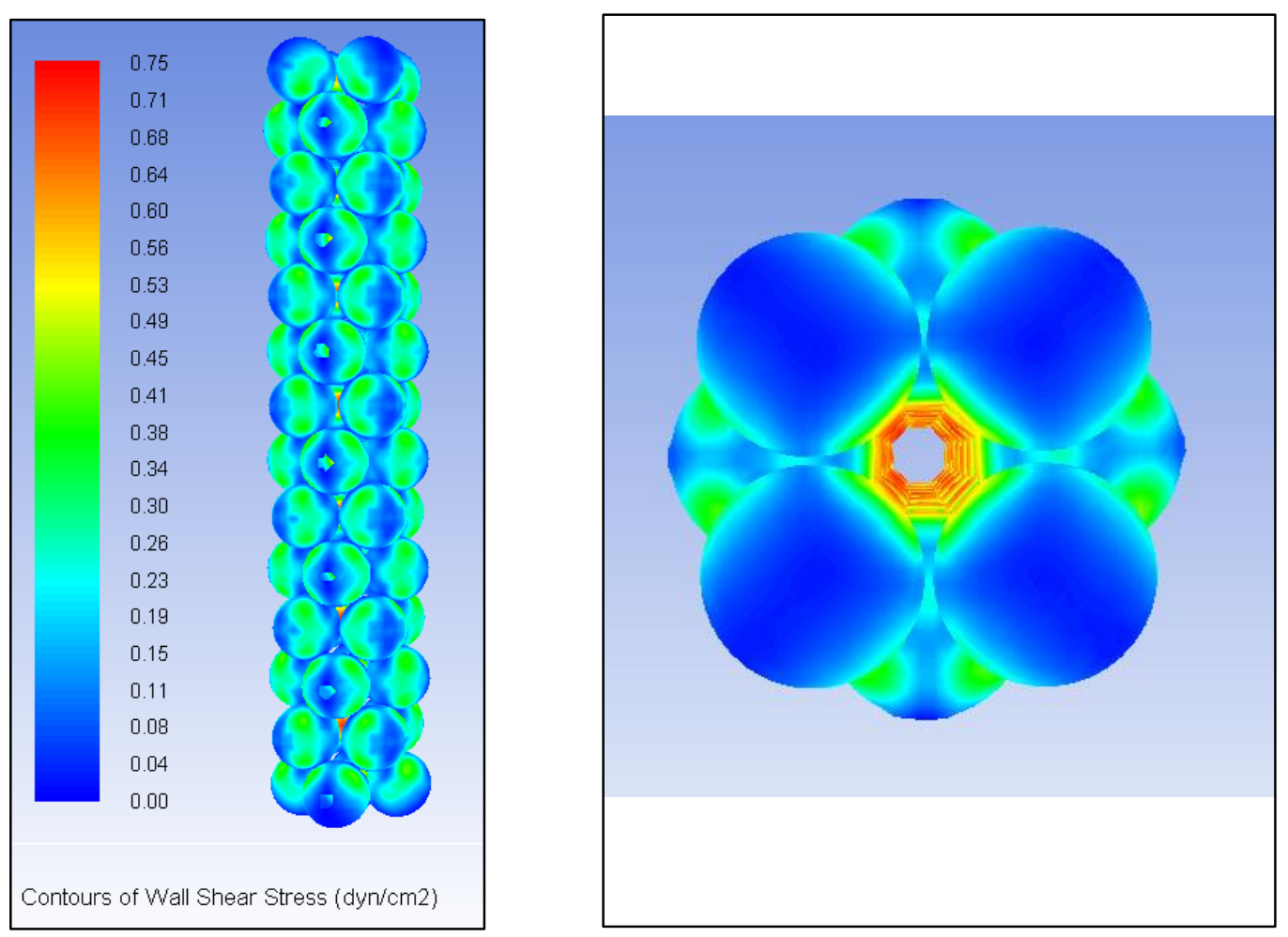

FIGURE 54 - Contour map of shear distribution on MC4 (D/d = 2.486) 


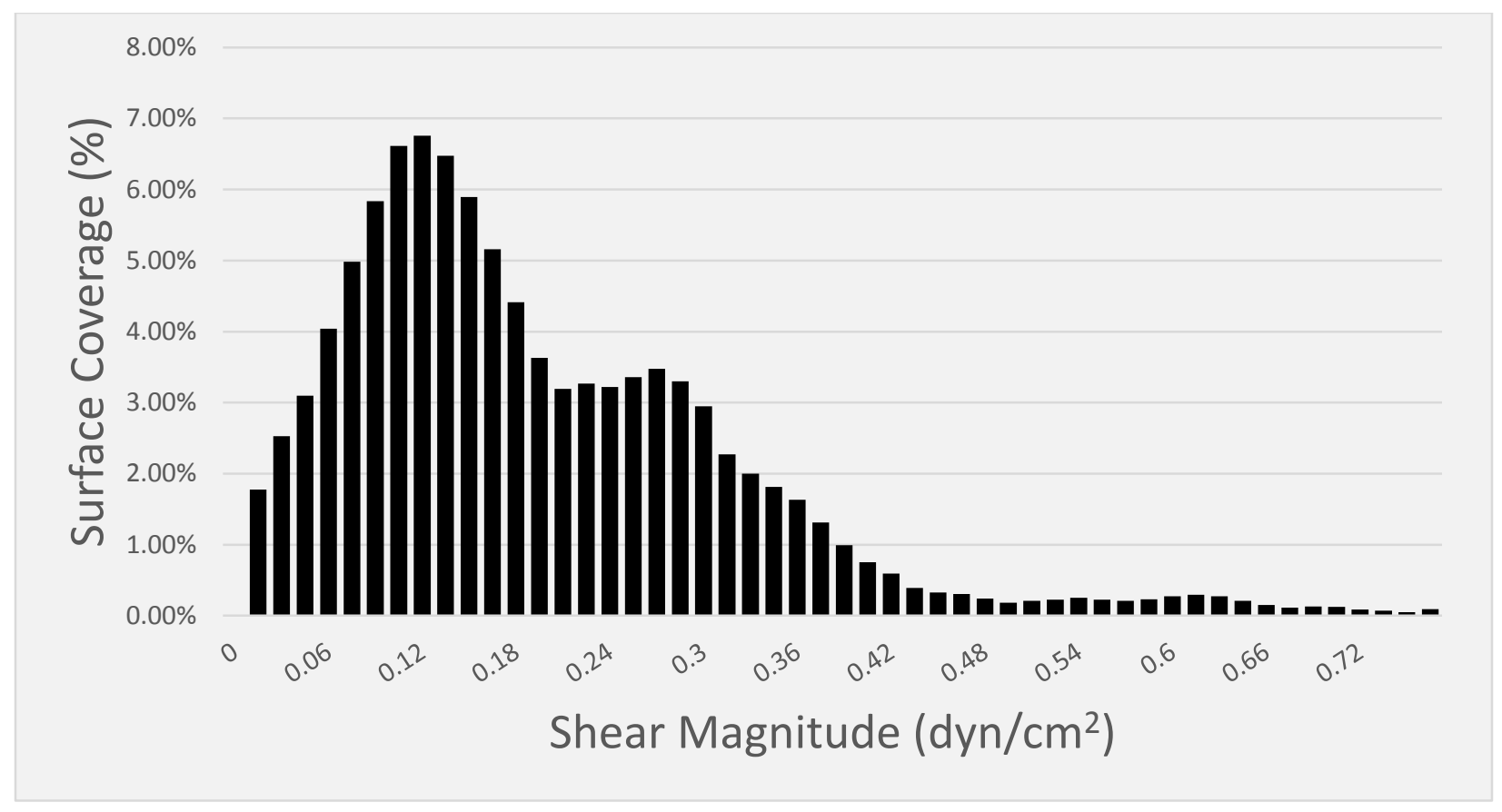

FIGURE 55 - Histogram of shear magnitudes for MC4 (D/d = 2.486)
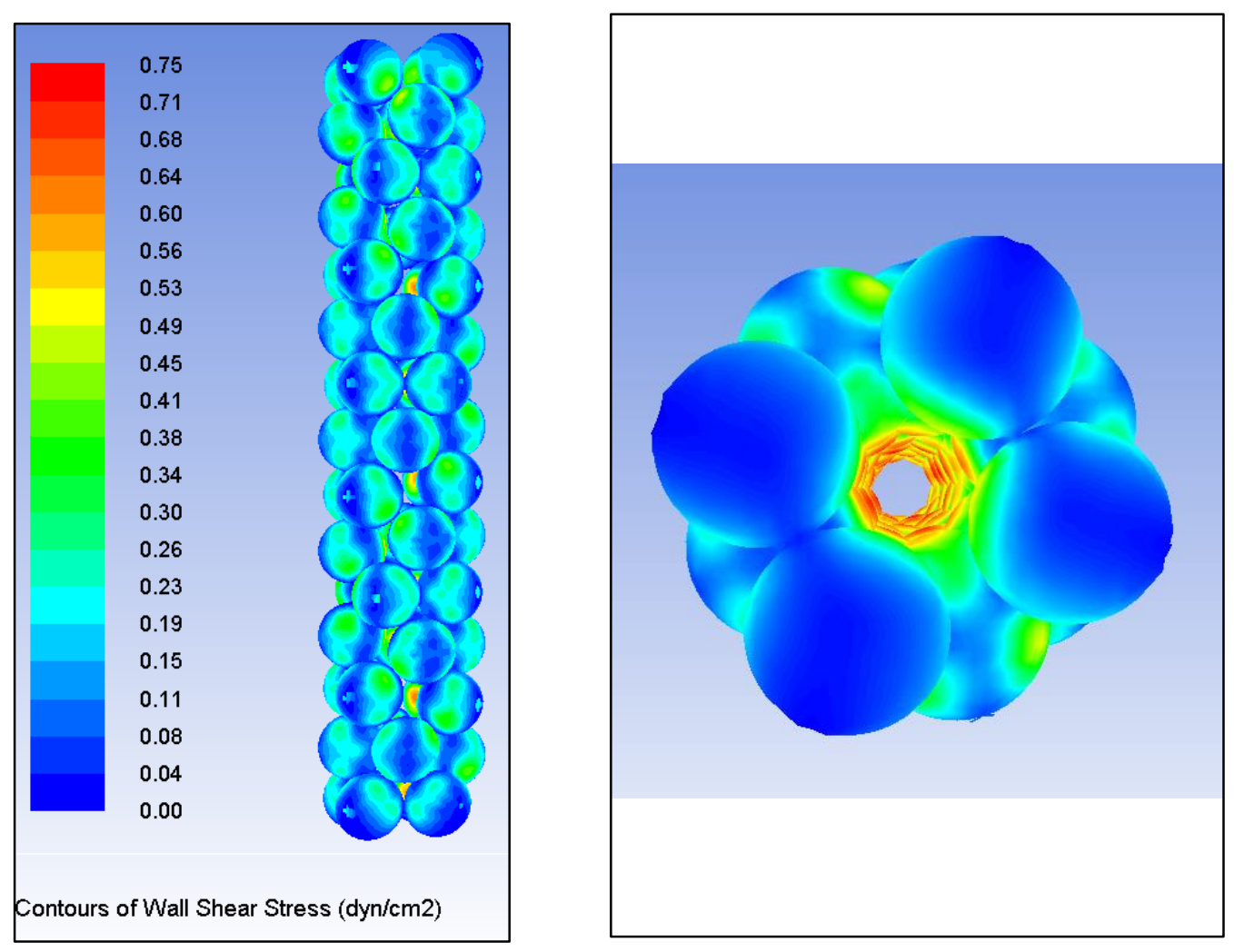

FIGURE 56 - Contour map of shear distribution on MC5 (D/d = 2.571) 


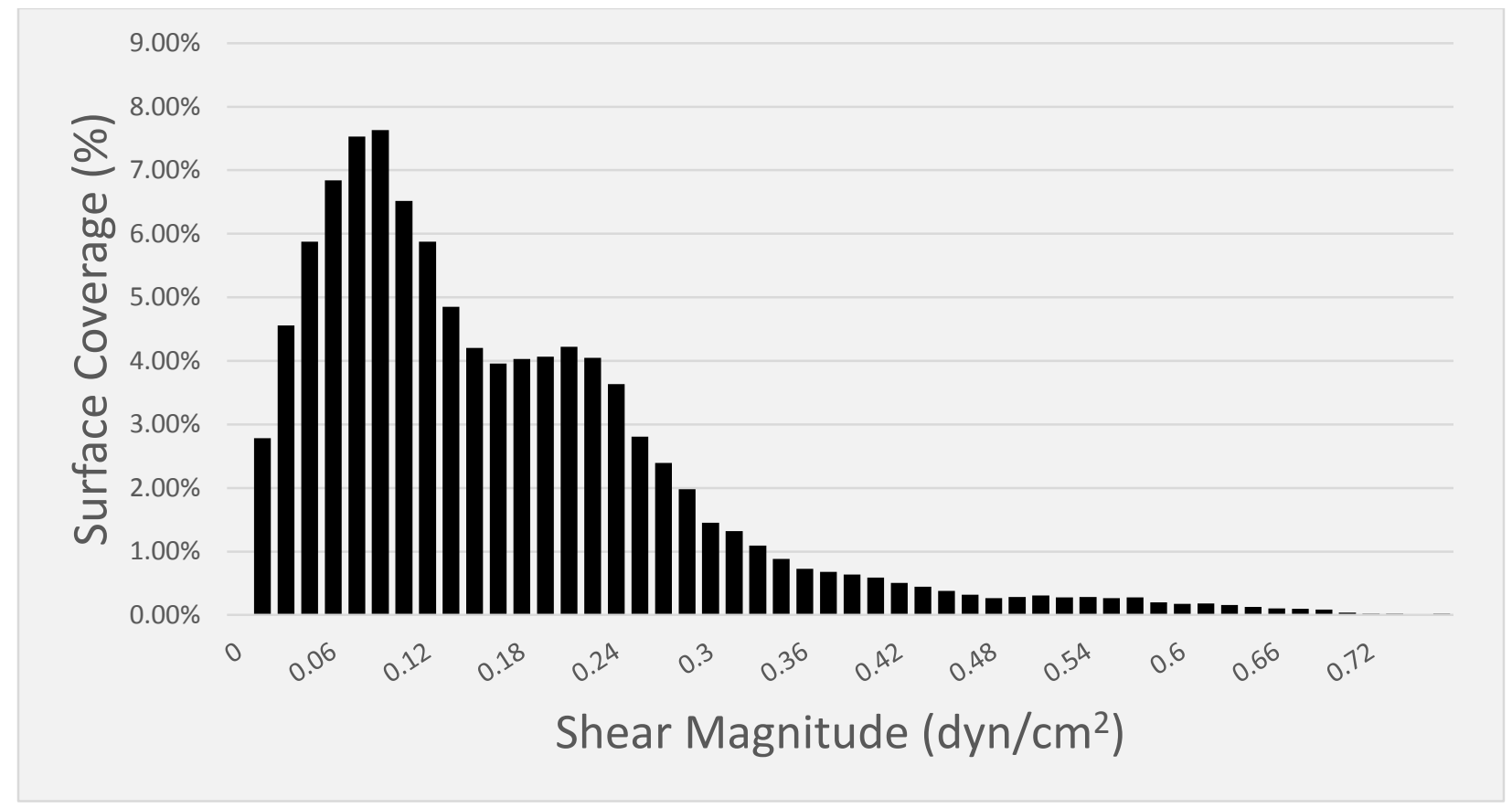

FIGURE 57 - Histogram of shear magnitudes for MC5 (D/d = 2.571)
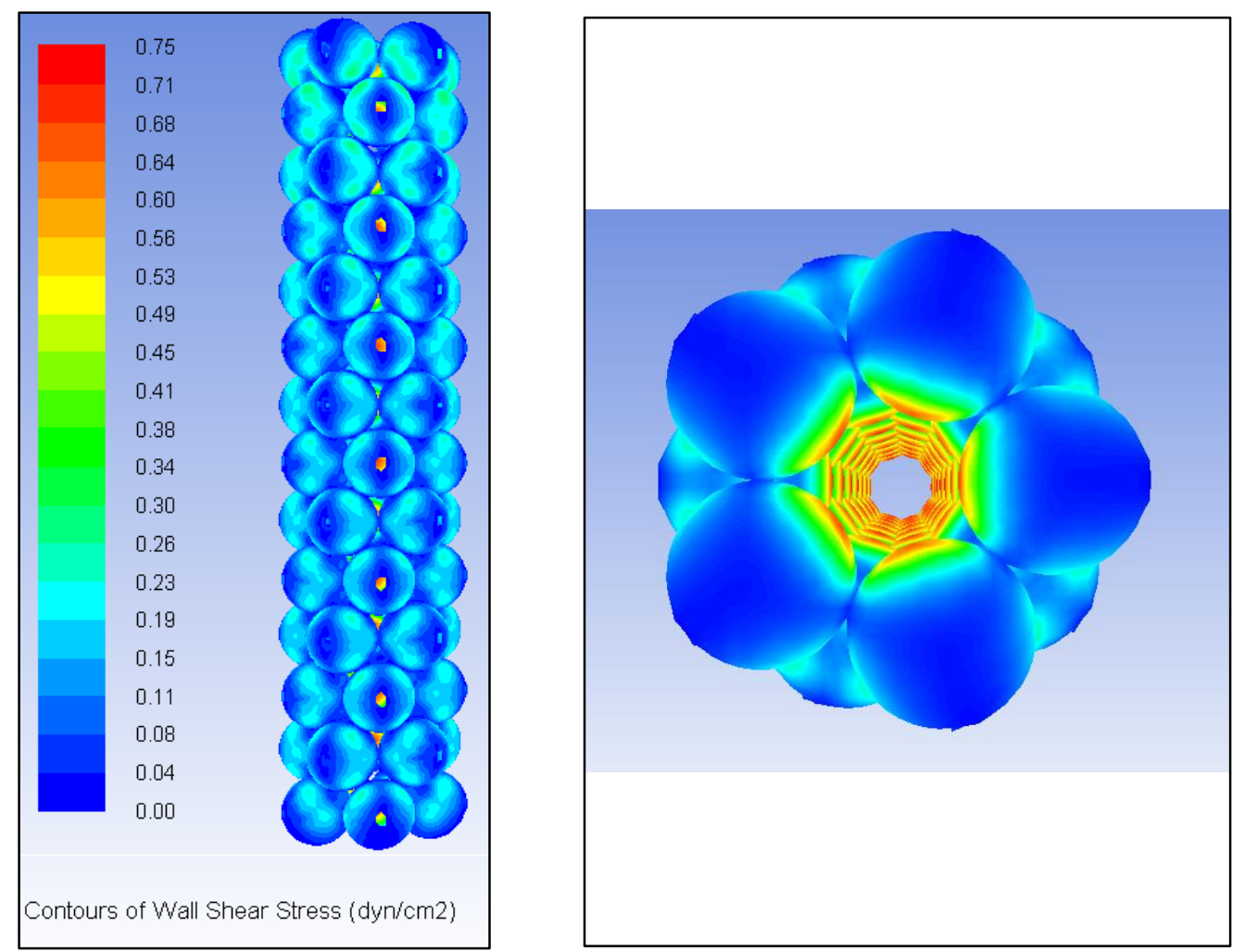

FIGURE 58 - Contour map of shear distribution on C5 $(\mathrm{D} / \mathrm{d}=2.701)$ 


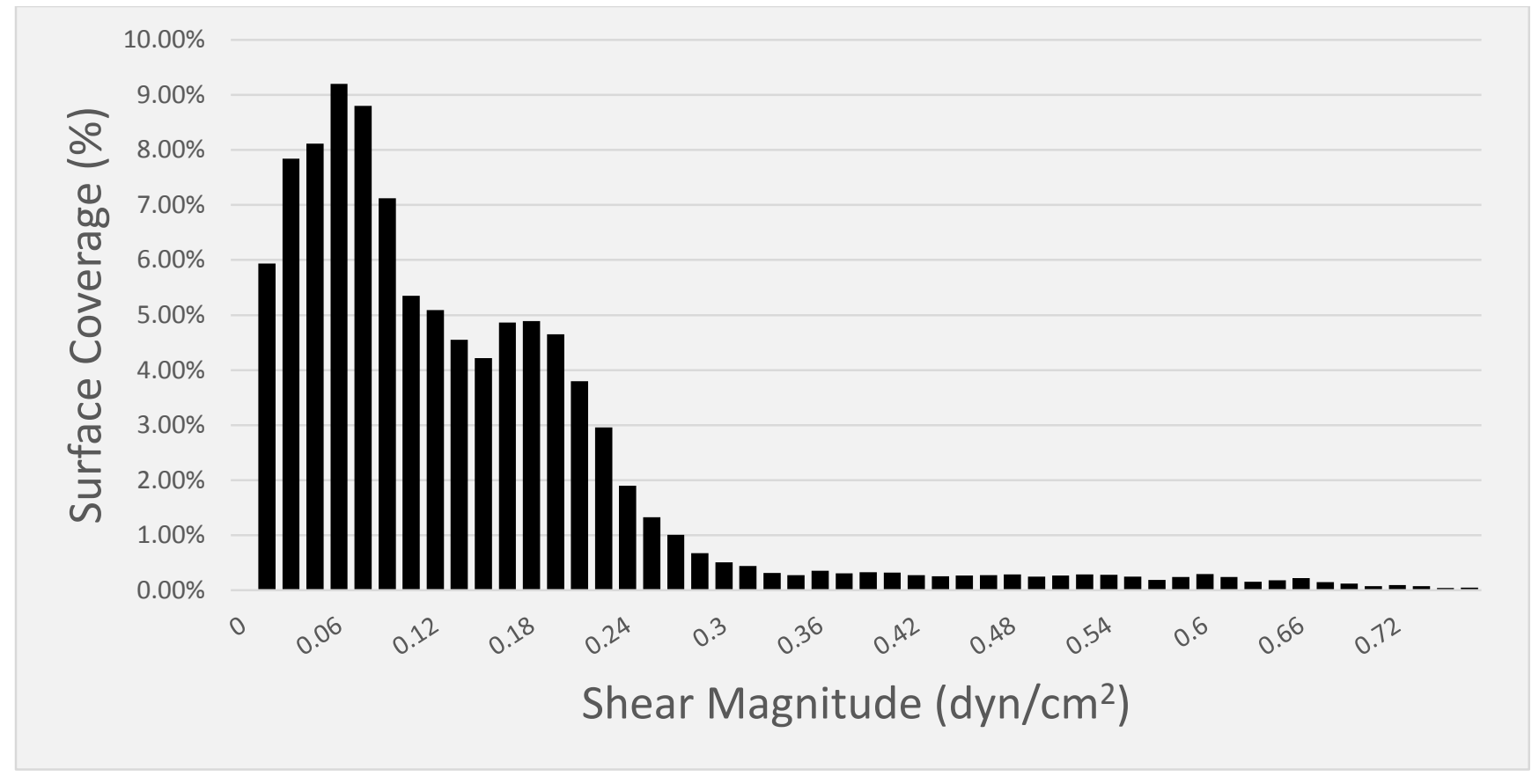

FIGURE 59 - Histogram of shear magnitudes for C5 $(\mathrm{D} / \mathrm{d}=2.701)$
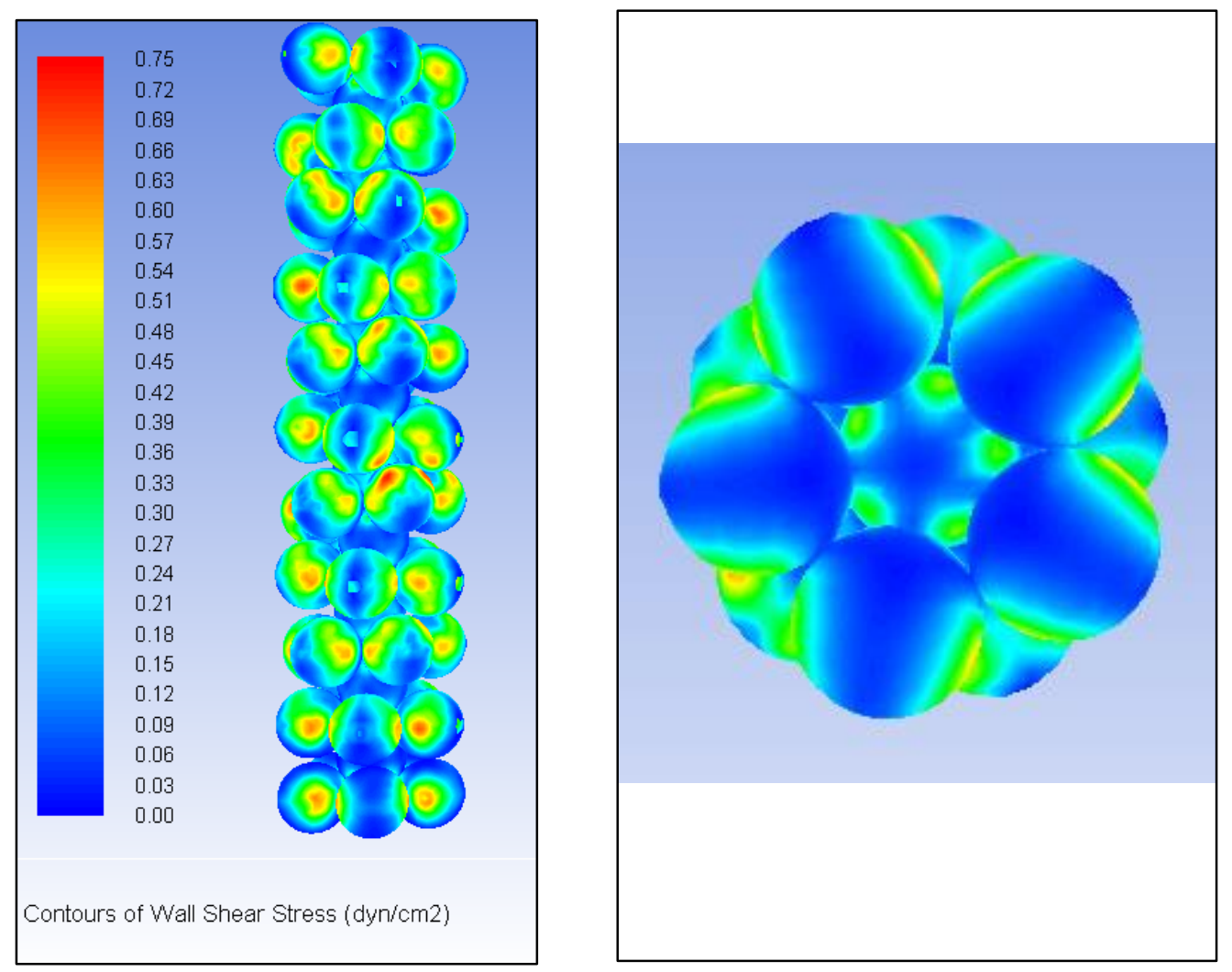

FIGURE 60 - Contour map of shear distribution on MC6 (D/d = 2.731) 


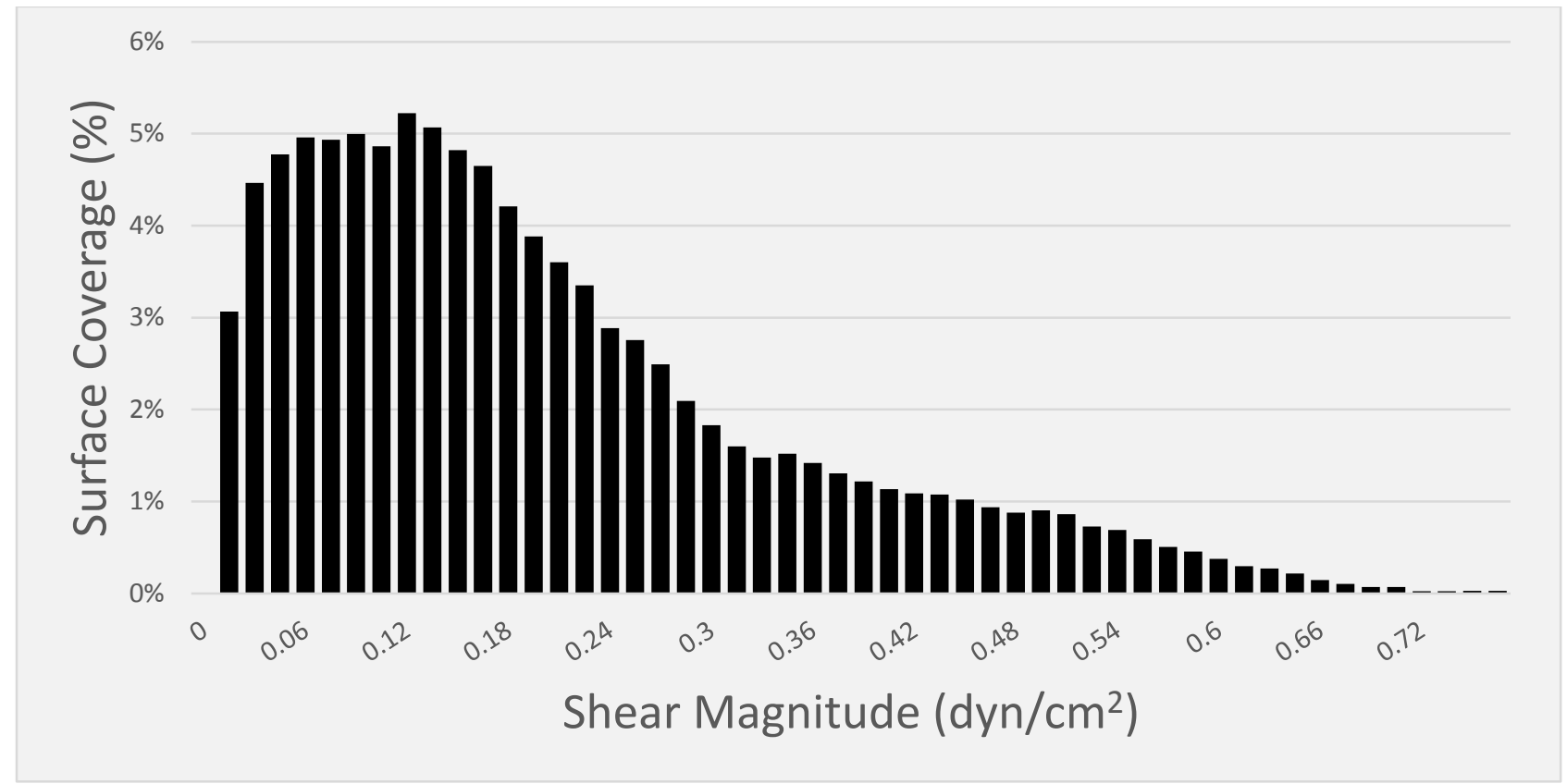

FIGURE 61 - Histogram of shear magnitudes for MC6 (D/d = 2.731)
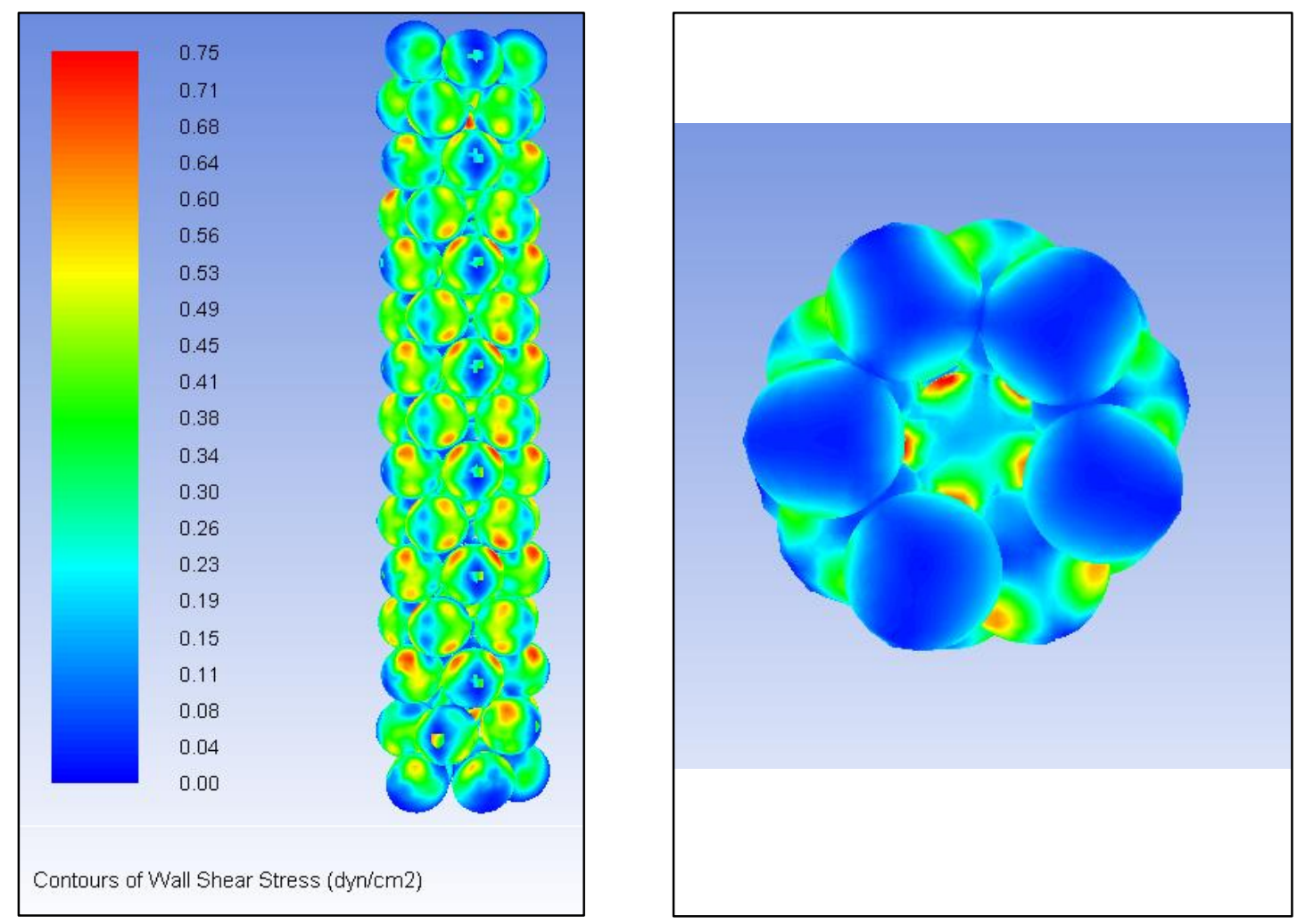

FIGURE 62 - Contour map of shear distribution on MC7 (D/d = 2.821) 


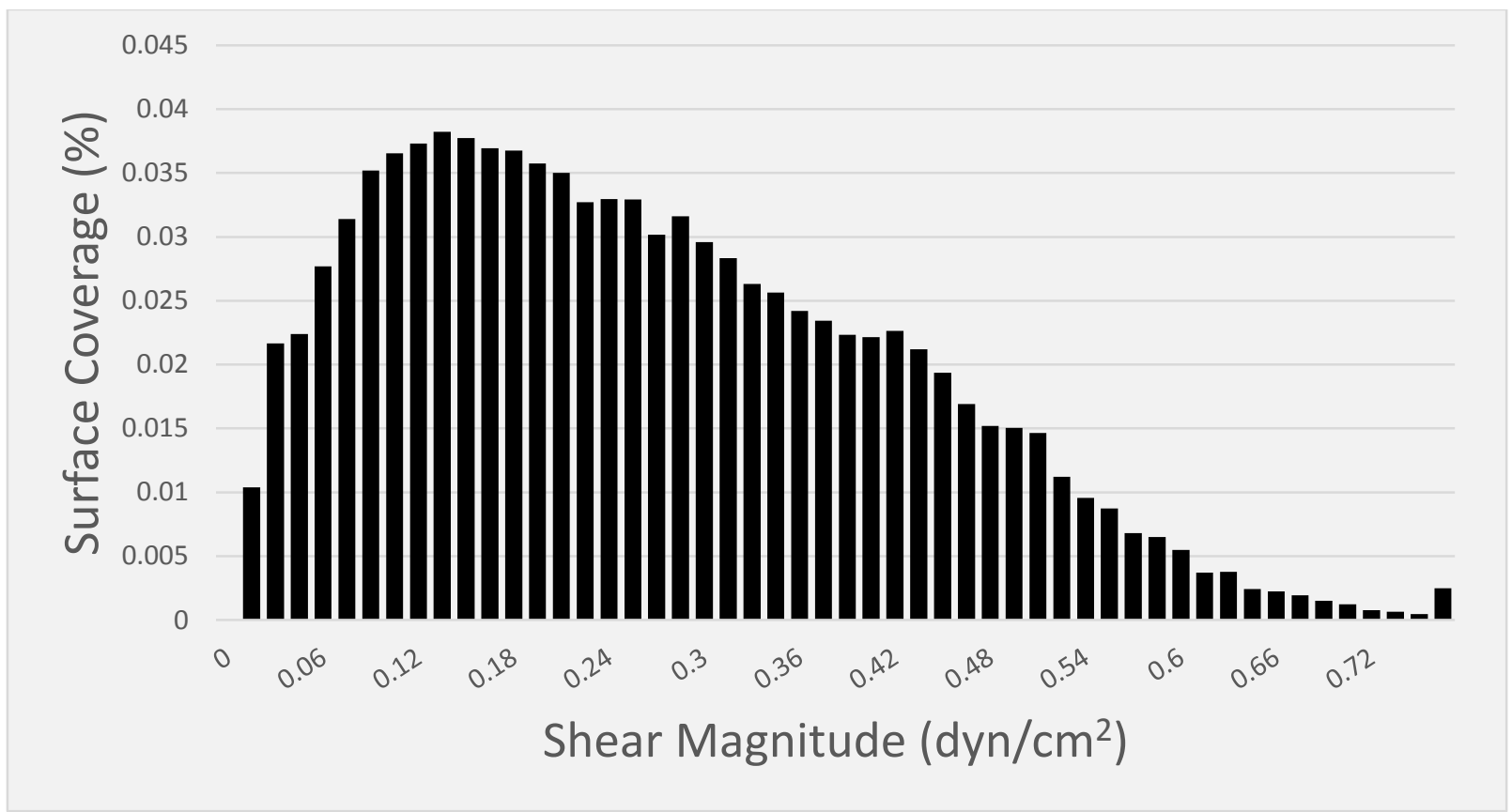

FIGURE 63 - Histogram of shear magnitudes for MC7 (D/d = 2.821)

Based on the histograms, the packing regimes simulated can be divided into three subsets. The first four packing regimes, $\mathrm{C} 2, \mathrm{MC} 1, \mathrm{C} 3$ and $\mathrm{MC} 2$, have no internal spheres and very little to no internal channel. In each case, a single mode is present, defined by a peak. As the inner channel begins to open up starting with MC3 and widening through $\mathrm{C} 4$, MC4, MC5 and C5, two peaks begin to develop, indicating a bimodal distribution. It is evident from the contour plots that one mode represents the average shear stress along the sides of the beads closest to the wall, while the higher peak represents the average shear stress within the hollow central cavity. MC6 and MC7, with the presence of internal spheres, define a third subset, in which the mode is less sharply defined, and shear coverage is more uniform throughout. A comparison between MC3 and MC7, the two packing regimes used in experimental trials, illustrates the individual variation in shear coverage as a result of differing geometry. 


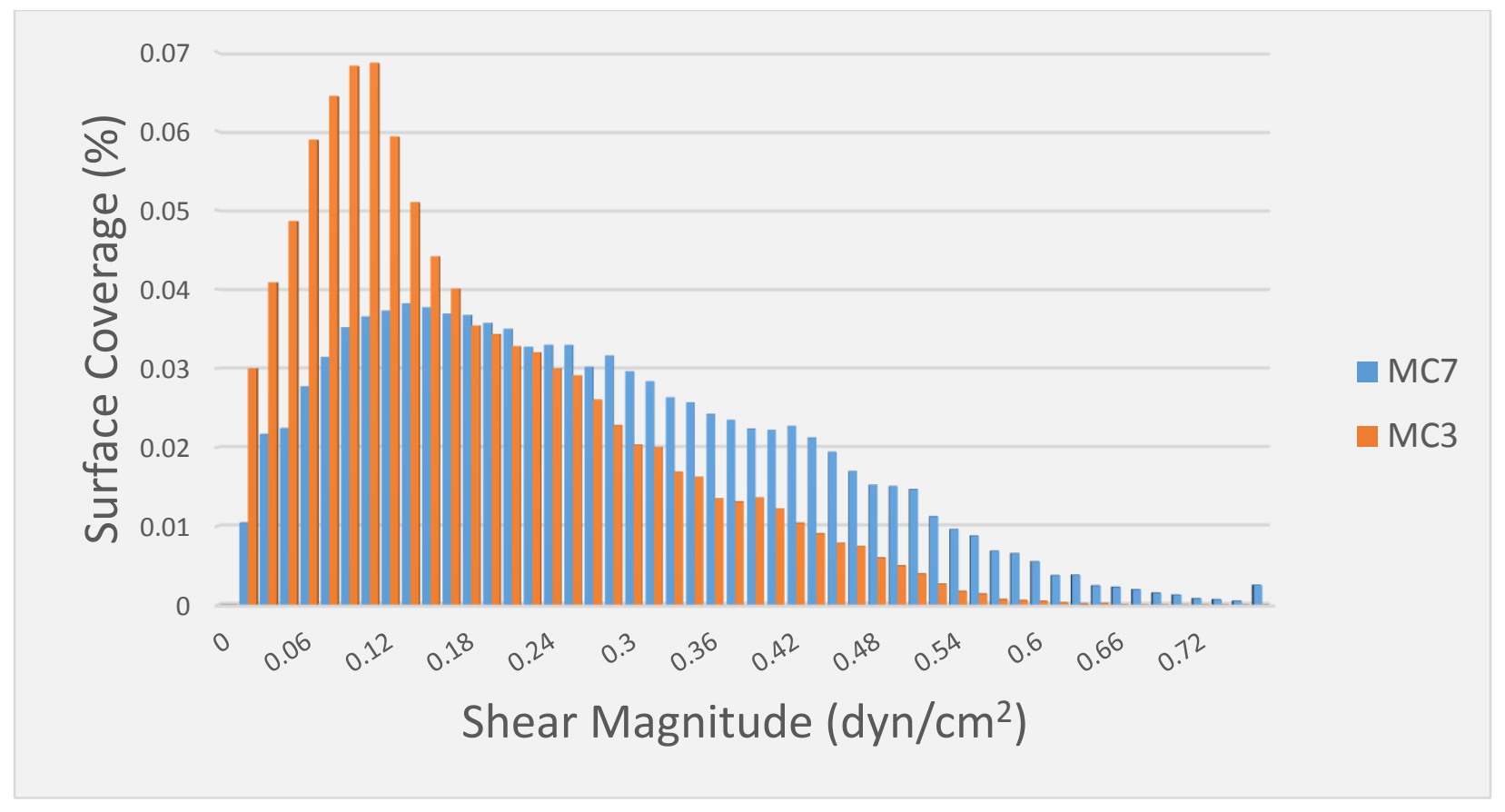

FIGURE 64 - Comparison of shear distributions between MC3 and MC7 packing regime

Because of the highly geometry-dependent nature of the shear stress distributions, comparing the mean shear stress over all bead surfaces is not an especially meaningful comparison. Additionally, since the signaling mechanisms at play within the bioreactor seem to act over a distance, it is more appropriate to compare the various packing regimes based on percentage of surface area at or above a certain shear stress level. Because Li et. al. identified $0.15 \mathrm{dyn} / \mathrm{cm}^{2}$ as a value at which OPN expression is enhanced, this value is chosen as the critical value. 


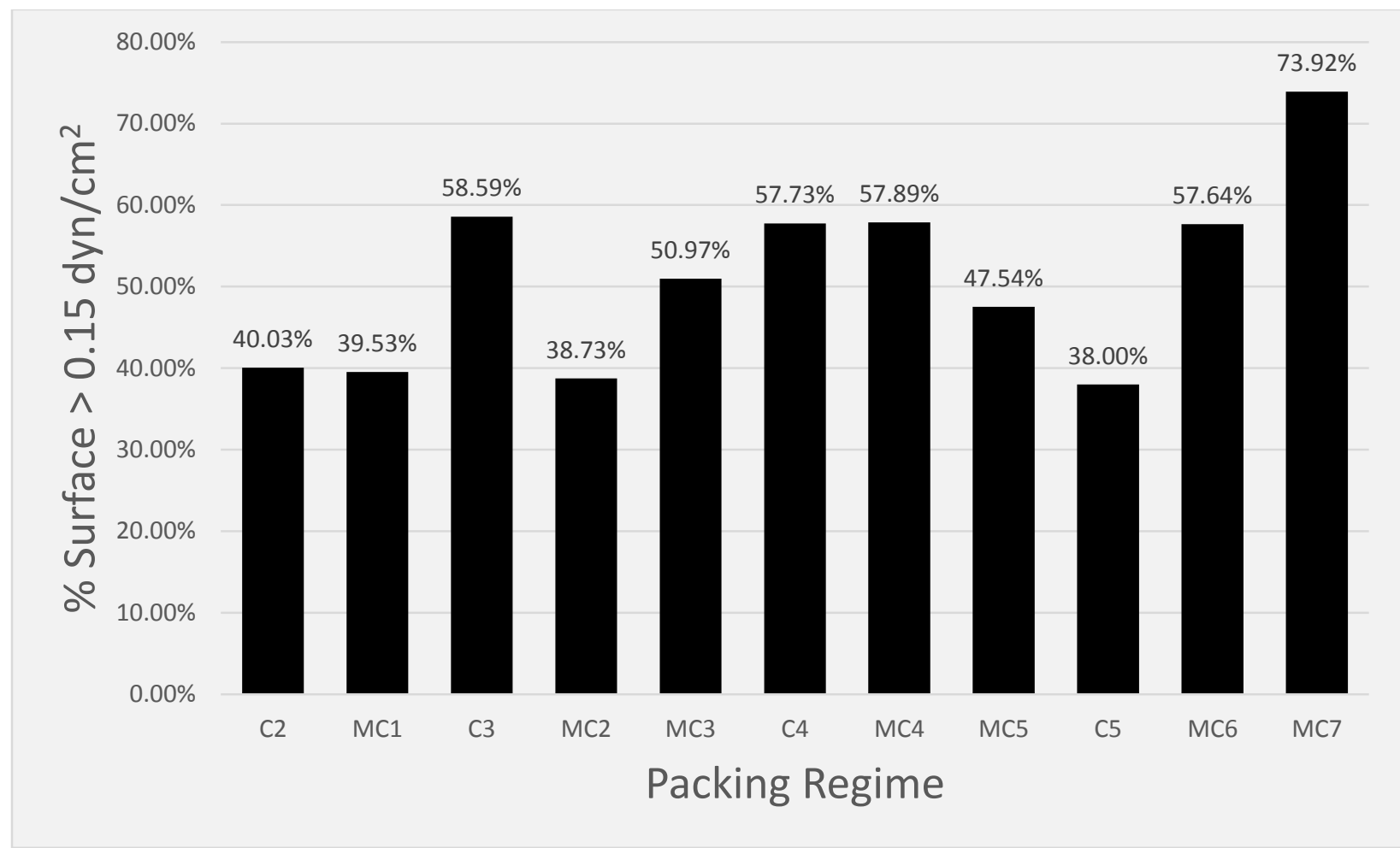

FIGURE $65-\%$ of surface $>0.15 \mathrm{dyn} / \mathrm{cm}^{2}$ for each packing regime

These data, taken together, suggest that selecting an optimal packing regime in order to maximize shear exposure is not always intuitive. The packing regimes containing interior spheres do ensure that a high proportion of the scaffold surface is covered with high shear levels. However, at lower D/d ratios, $\mathrm{C} 3$ demonstrated the highest average shear and percentage of surface area at or above $0.15 \mathrm{dyn} / \mathrm{cm}^{2}$. Conversely, MC2 proved to be a particularly poor arrangement, due to the nature of the packing in which nearly all of the beads are in close contact with the wall. This reinforces the idea that the average shear experienced along the scaffold surfaces is highly geometry-dependent. 


\section{Advective Flow and Nutrient Transport}

These trials were conducted under the same operating conditions as the shear stress trials, in order to determine the magnitude of advective flow within the scaffold particles as a function of packing regime, as well as the spatial distribution of advective flow, i.e. how deep media flow is able to penetrate beyond the scaffold surface. The relative contributions of advective and diffusive flow were quantified by the Peclet number. All Peclet number calculations were performed using the diffusion coefficient of oxygen in alginate, $2.56 \times 10^{-9} \mathrm{~m}^{2} / \mathrm{s}$ (Fisher et. al., 2011). The choice of characteristic length has a significant effect on the magnitude of the calculated Peclet number. Because the scope of the study is to measure nutrient transport across the entirety of the scaffold, the bead diameter was chosen as the characteristic length. However, if the mean pore diameter were selected as characteristic length, the calculated Peclet number would be orders of magnitude lower. As before, the first analysis was a comparison of average Peclet number over the entire bead region. Based on this data, a determination of whether or not transport in the bioreactor is largely advection based or diffusion based could be made. However, further analysis was necessary to determine spatial variations in the magnitude of advective flow through the scaffold particles.

According to Huysmans and Dassargues (2004), Peclet number values much less than 1 typically indicates a region in which diffusion plays a more significant role than advection with regards to species transport. In cases where size exclusion effects prevent access to most of the porous volume, however, very high Peclet numbers do not necessarily indicate that advective transport is predominant. Special definitions of the Peclet number including an effective porosity are available for this case. However, in this study, oxygen is the model species, and thus size exclusion effects are negligible. The critical Peclet number values defined for this study were 0.1 and 1.0. These values were largely chosen for comparison across different trials, but are not 
entirely arbitrary. For a small species such as oxygen, advection and diffusion are traditionally considered to contribute equally to overall mass transport at $\mathrm{Pe}=1.0$. Likewise, $\mathrm{Pe}=0.1$ would correspond to $1 / 10$ the overall contribution at $\mathrm{Pe}=1.0$, or $5 \%$ of overall mass transport.

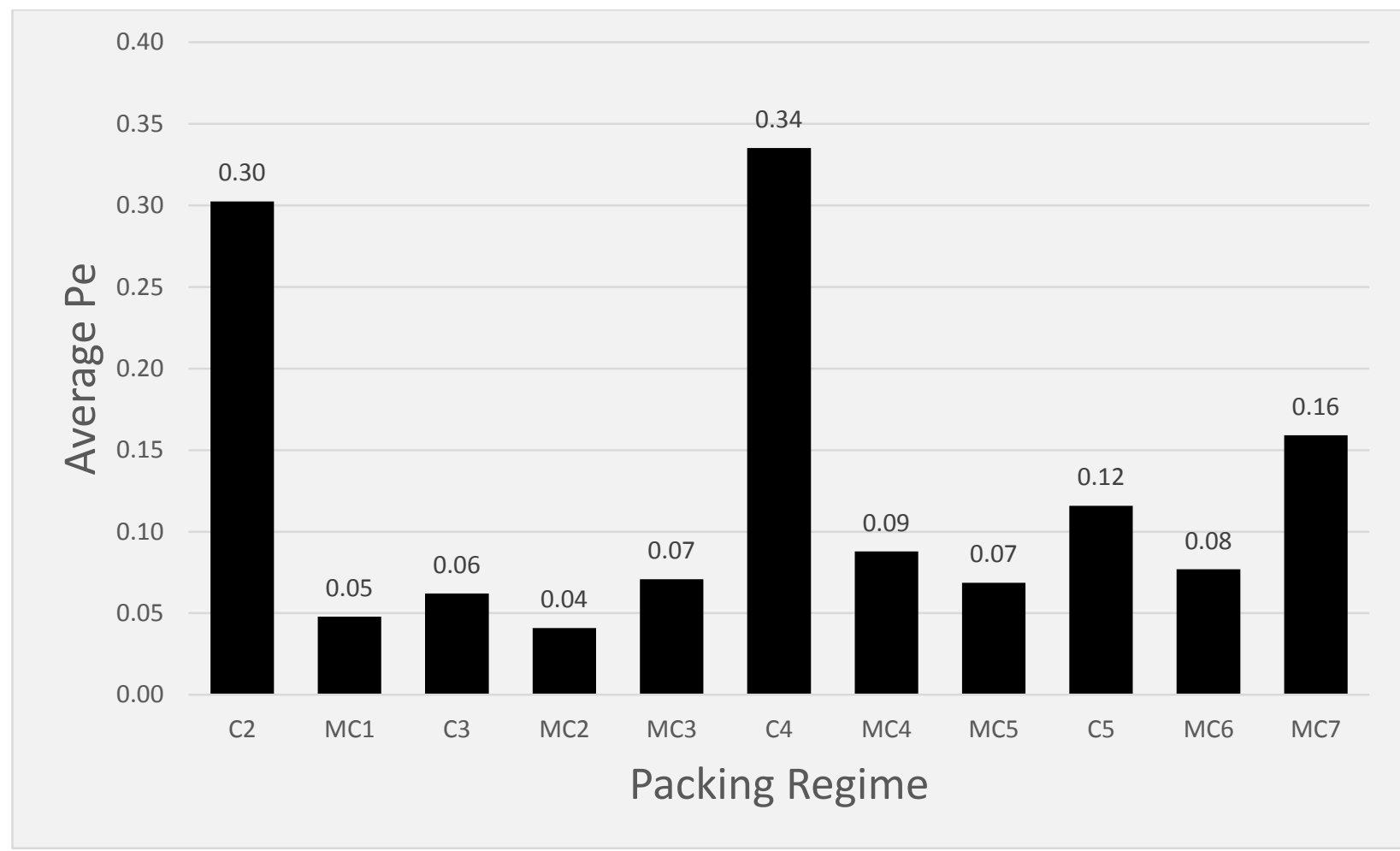

FIGURE 66 - Average Peclet number as a function of packing regime

Based on the critical values of Peclet number defined in the preceding paragraph, FIGURE 66 suggests that in all cases, diffusion contributes more significantly to overall mass transport than advection. Curiously, C2 and C4 exhibit a significantly higher average Peclet number than all other packing regimes. These findings were originally approached with skepticism. If a small portion of the scaffold experiences an extremely high rate of flow, this could significantly skew the average. Additionally, unreasonably high velocity values could suggest poor solution convergence in Fluent. In order to investigate, histogram plots were created comparing the $\%$ of scaffold volume at or above $\mathrm{Pe}=0.1$ and $\mathrm{Pe}=1.0$ for each packing regime. 


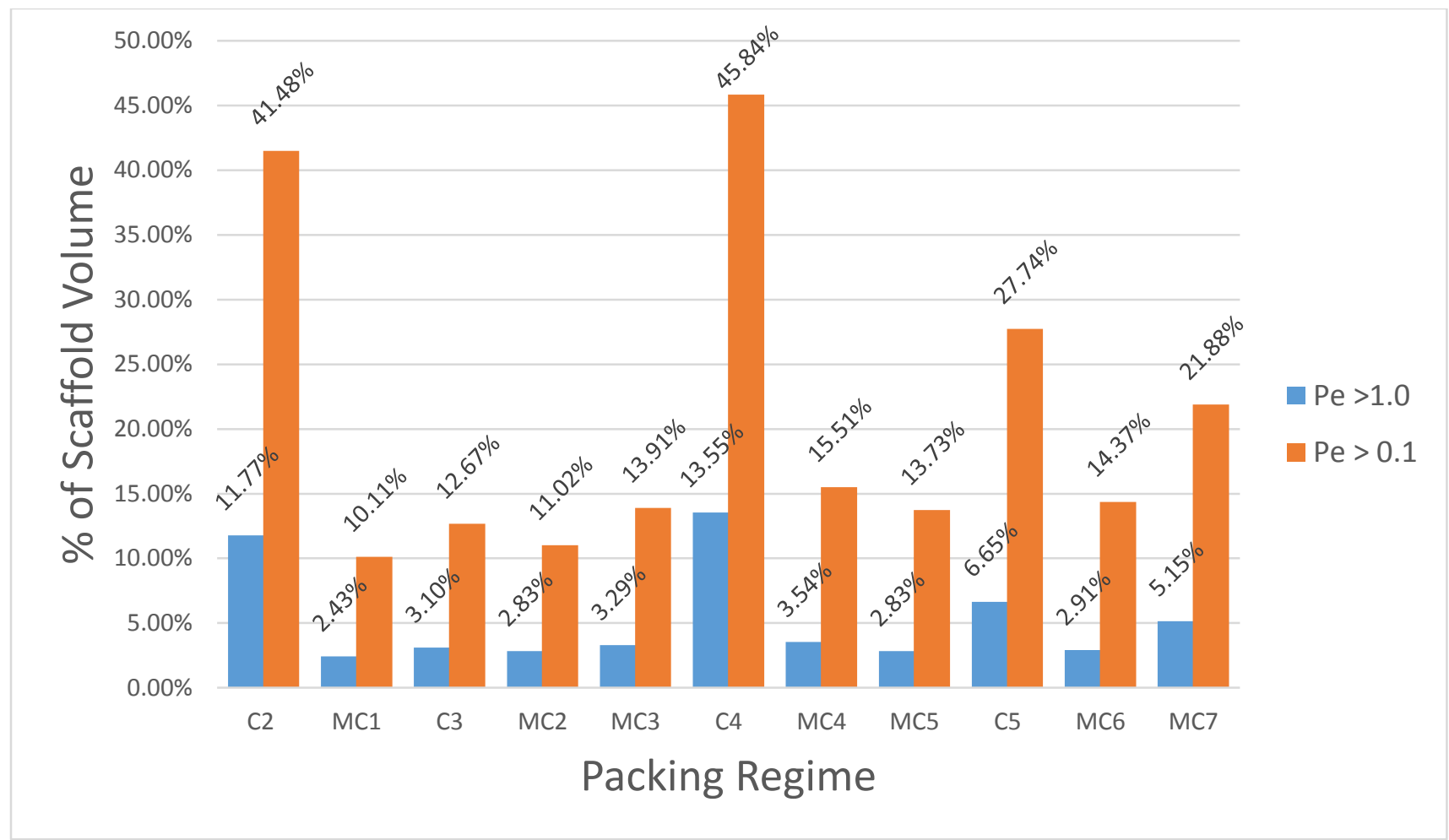

FIGURE 66 - Average Peclet number as a function of packing regime

This data is consistent with the average Peclet number findings and confirms that the averages are not skewed by the presence of small pockets with extremely high velocity. A plausible physical explanation for this finding is that the nature of $\mathrm{C} 2$ and $\mathrm{C} 4$ is such that each unit cell is rotated $90^{\circ}$ relative to the one prior. This means that the flow stream strikes each bead perpendicular to its surface, forcing the greatest amount of flow through the pores of the scaffold. Additionally, due to the highly symmetrical nature of both geometries, each bead is subjected to the same flow pattern, whereas in other packing regimes there may exist beads which contact relatively little of the flow stream. The velocity field in C4 is illustrated in FIGURE 65. 


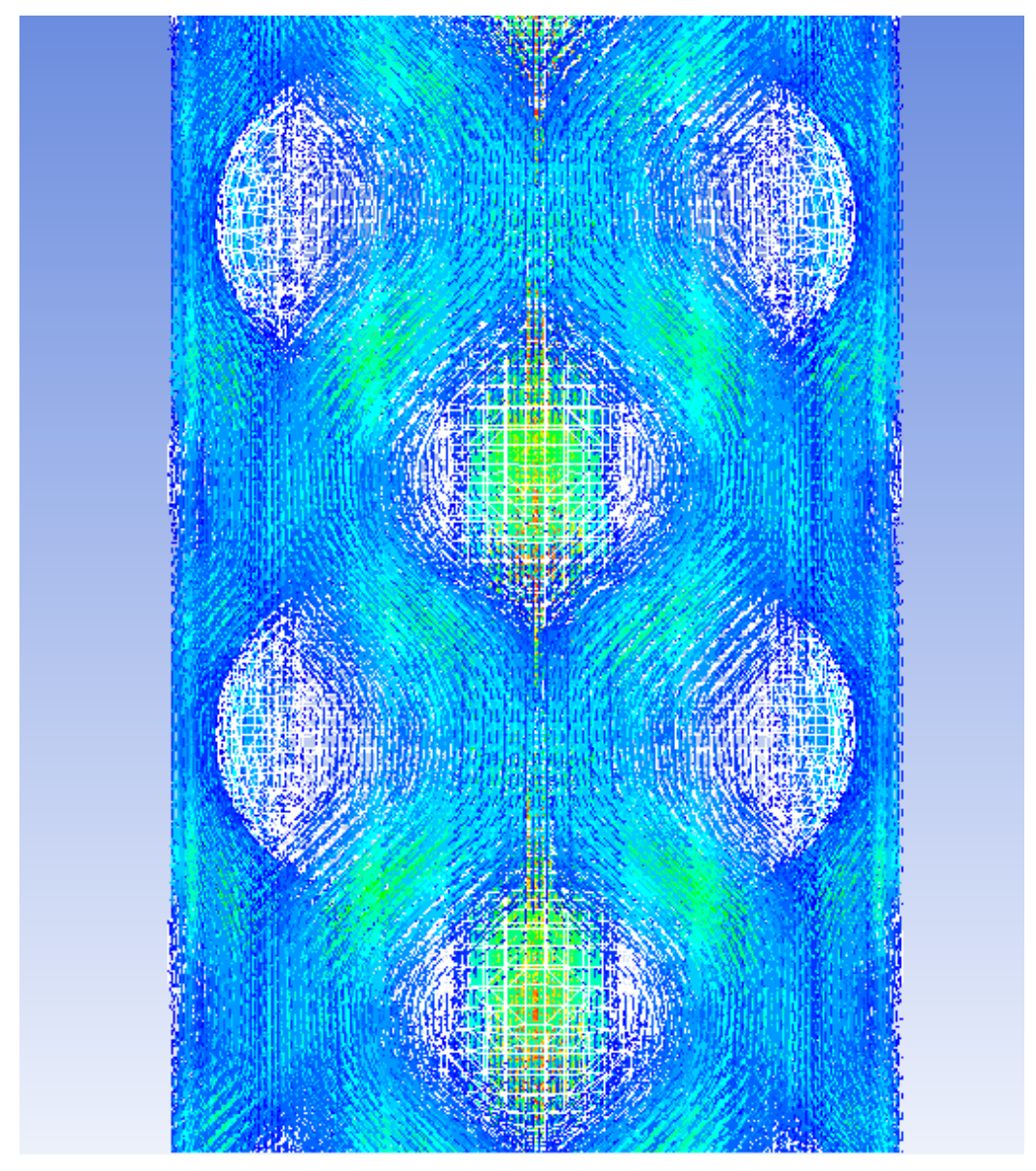

FIGURE 68 - Vector plot for C4 showing flow striking beads perpendicular to surface

To determine if the flow pattern unique to $\mathrm{C} 2$ and $\mathrm{C} 4$ caused the drastic increase in advective flow through the scaffold region, cross sections were taken from representative beads within the structure and the velocity fields within the beads were analyzed. The following figures show cross sections of individual beads within $\mathrm{C} 2$ and $\mathrm{C} 4$ and illustrate regions where $\mathrm{Pe}>1$. This shows the effective penetration depth of the growth media within the scaffold material. 


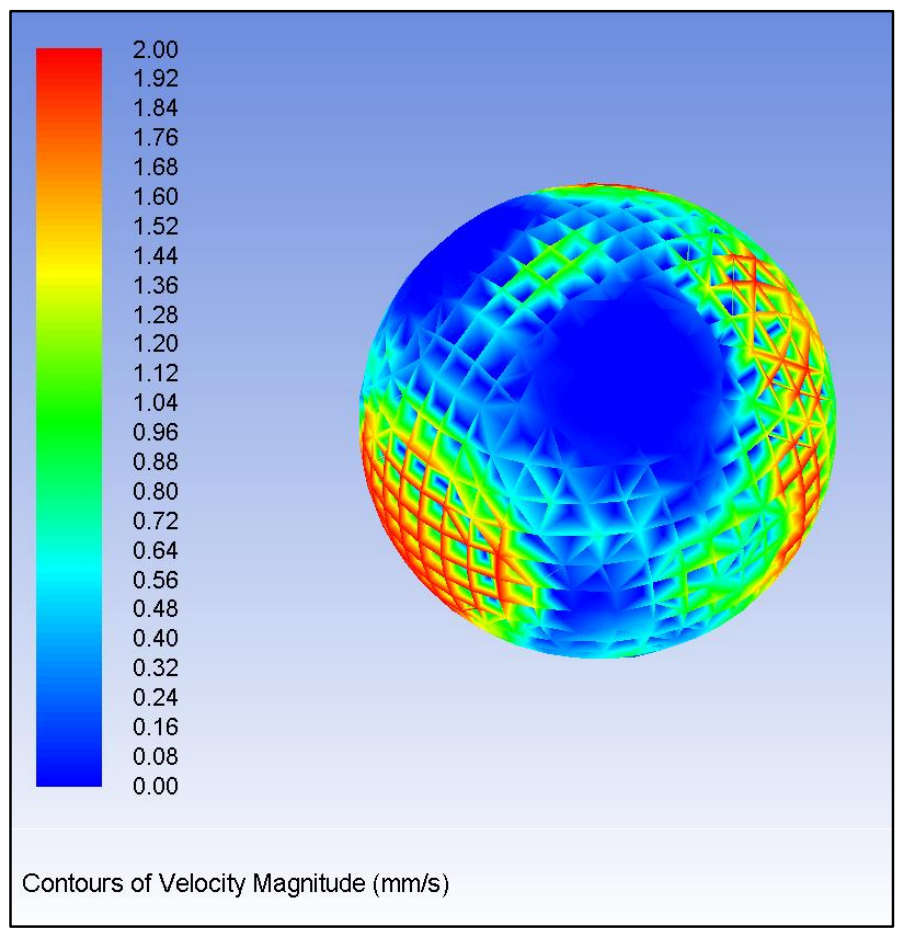

FIGURE 69 - Velocity Contours on representative bead surface for C4
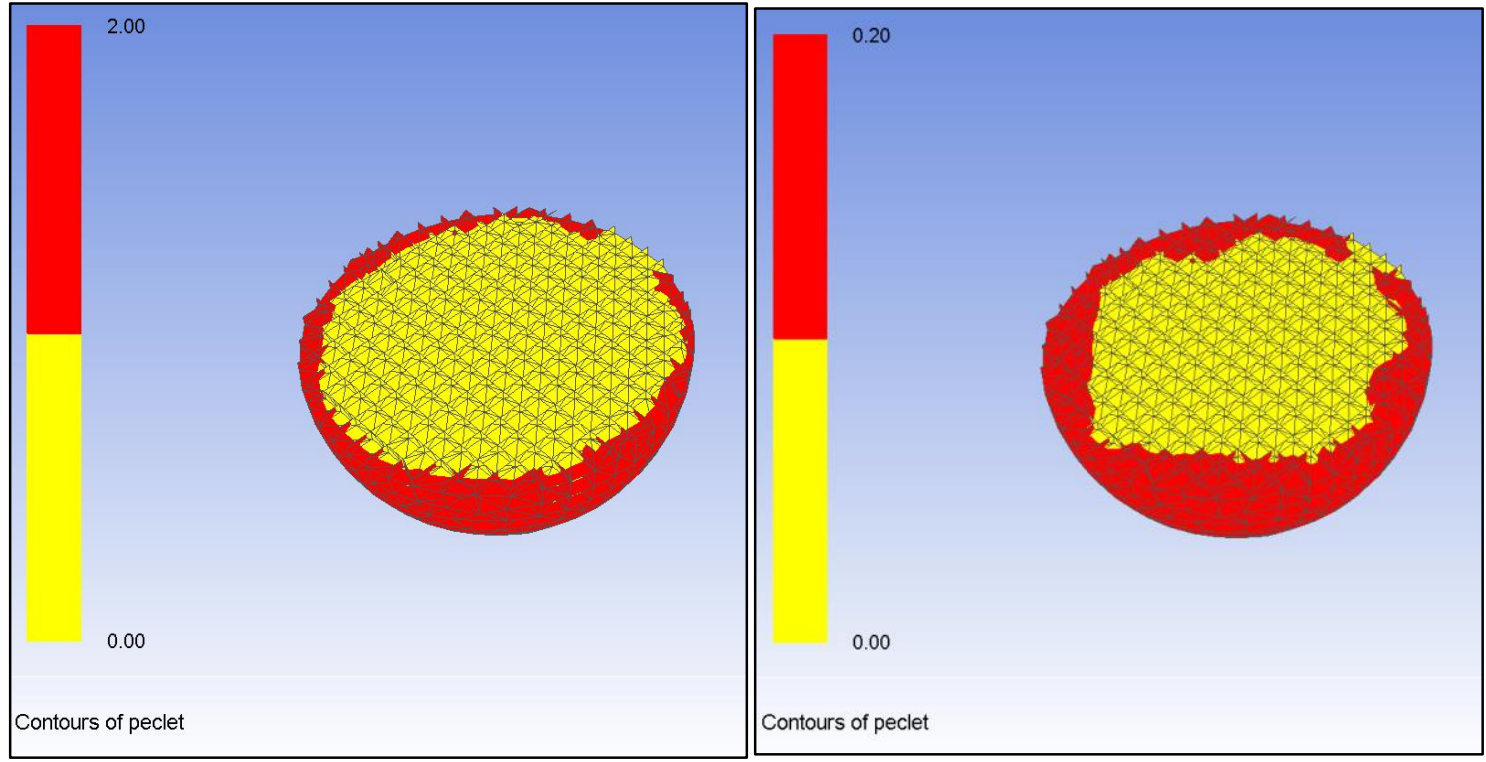

FIGURE 70 - Cross section of C4 bead showing Pe $>1$ (left) and Pe $>0.1$ (right) 


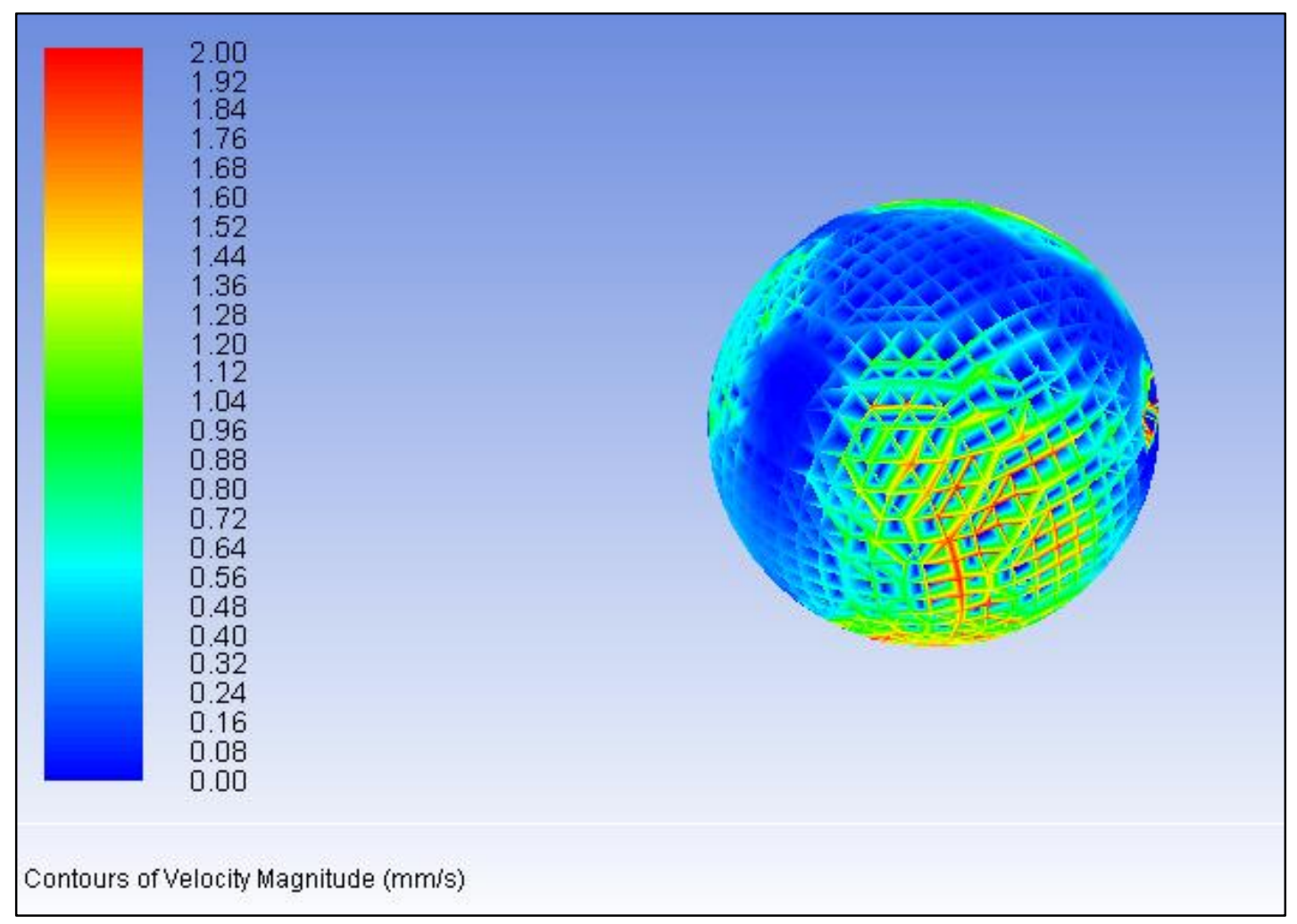

FIGURE 71 - Velocity Contours on representative bead surface for C2
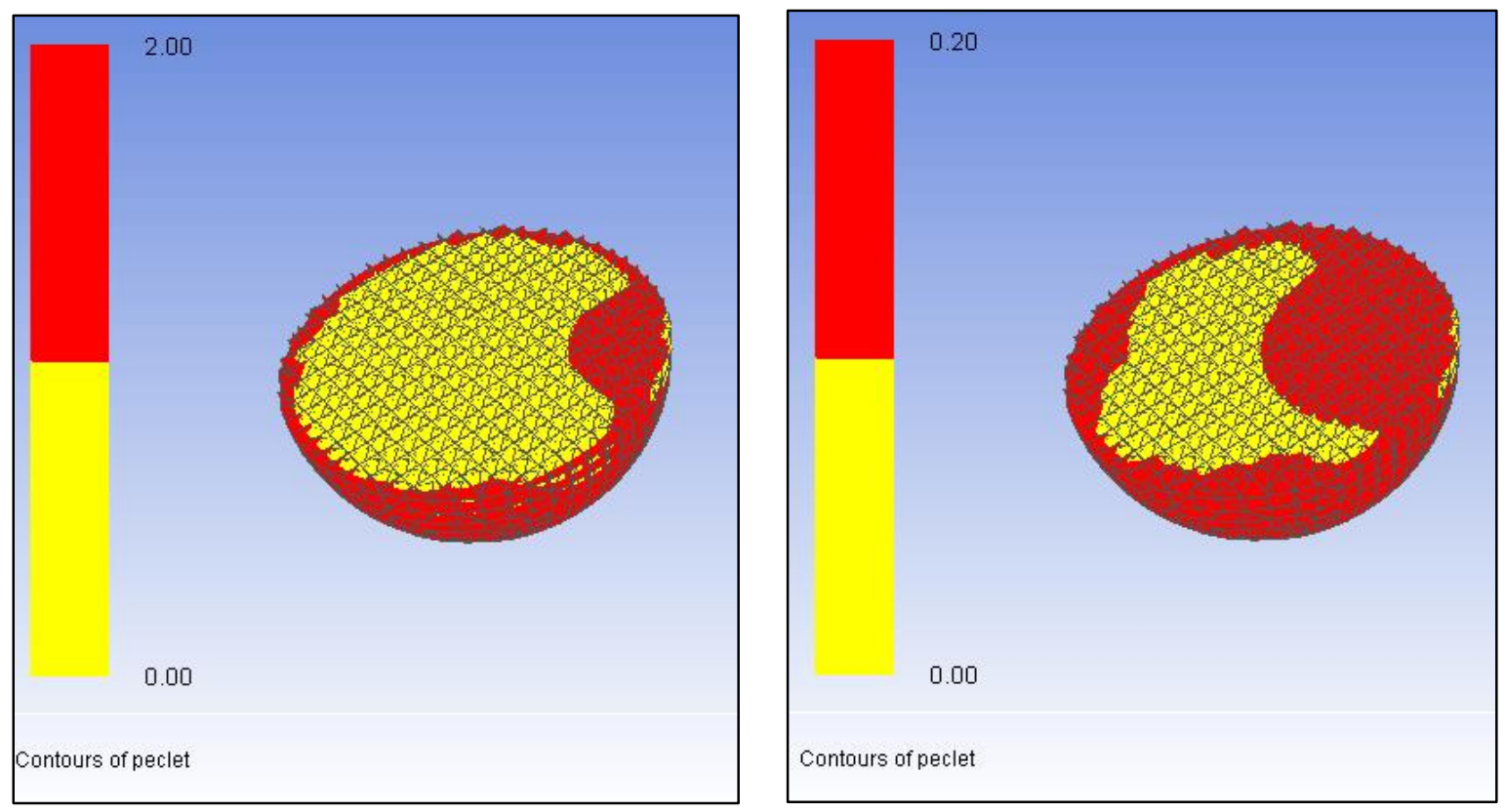

FIGURE 72 - Cross section of C2 bead showing Pe $>1$ (left) and Pe $>0.1$ (right) 
This data confirms that for alginate scaffolds, advective flow only influences nutrient transport significantly in the vicinity of the bead surface. Alginate is a very tightly porous material, so this result is as expected. It also suggests that the packing regimes corresponding to $\mathrm{C} 2$ and $\mathrm{C} 4$ carry an advantage over other regimes due to the nature of their geometry. This is somewhat counterintuitive, since these beads are larger in size than in other packing regimes tested. It therefore seems as though there would be more resistance to nutrient transport, as the center of the bead is farther removed from the bulk media flow. However, the simulation suggests that this is not the case. Even when normalized by comparing \% volumes at different Peclet numbers, C2 and C4 carry a distinct advantage, thought to be due to their unique symmetry.

\section{Flow Rate Comparison}

The same analysis performed in the previous paragraphs was repeated for $10 \mathrm{~mL}$ per minute to determine how altering the flow rate affects shear stress and nutrient transport. For these cases, the packing regimes MC3 and MC7 were chosen, as they correspond closely with the packing regimes used in experimental trials. As before, average shear stress, histogram plots, average Peclet number and Peclet number distribution are presented for each case at 3 and $10 \mathrm{~mL} / \mathrm{min}$. 


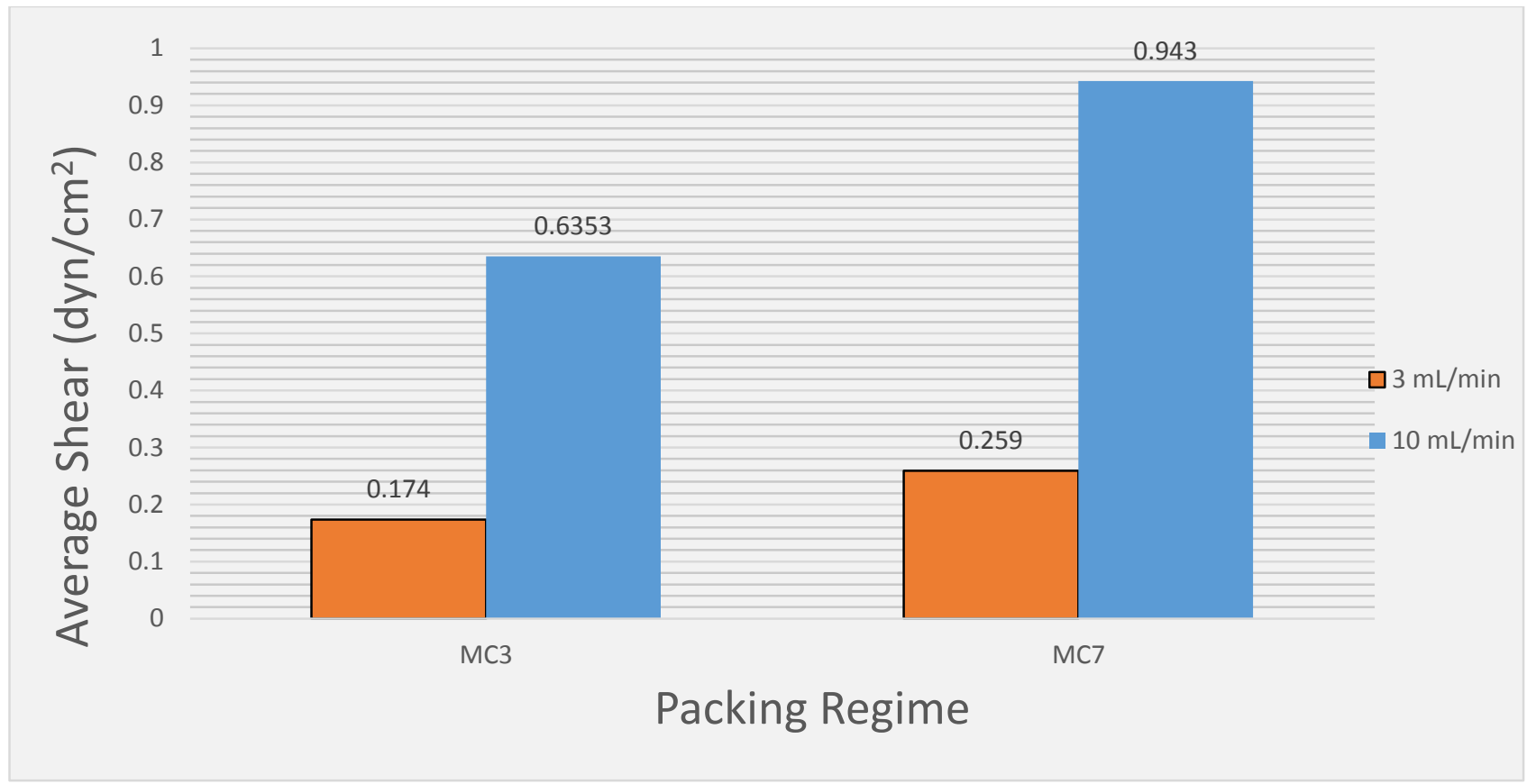

FIGURE 73 - Average shear stress values for MC3 and MC7 at 3 and $10 \mathrm{~mL} / \mathrm{min}$

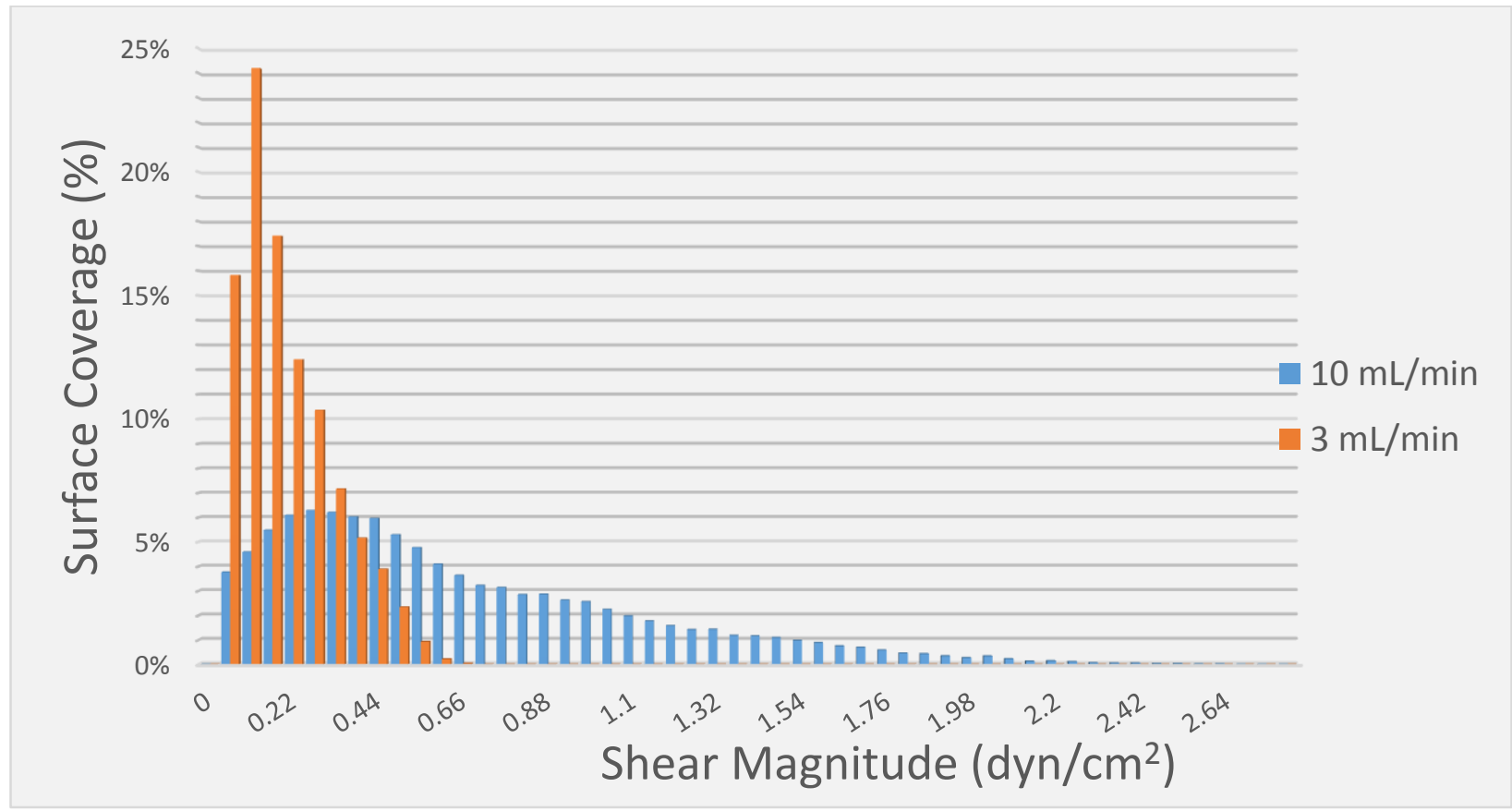

FIGURE 74 - Shear stress distributions for MC3 at 3 and $10 \mathrm{~mL} / \mathrm{min}$ 


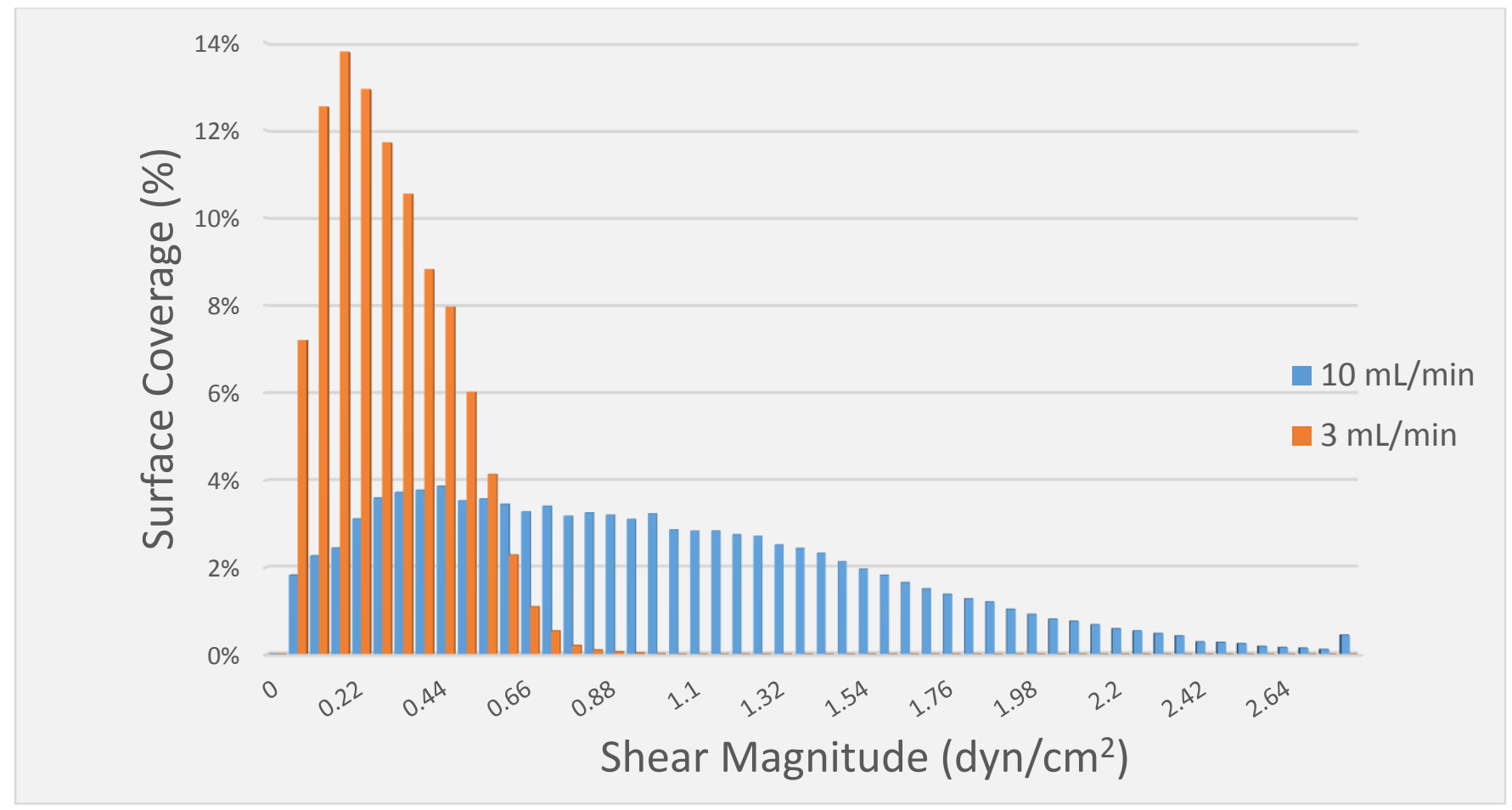

FIGURE 75 - Shear stress distributions for MC7 at 3 and $10 \mathrm{~mL} / \mathrm{min}$

In both geometries, a 3.3-fold increase in flow rate elicited an increase in average shear stress greater than 3.3-fold. For MC3, the average shear stress increased by a factor of 3.75 and for MC7 the factor was 3.64. Despite the higher average shear stress values, still very little of the scaffold surface in either case experienced a shear stress magnitude greater than the critical value for OPN expression determined by Kreke et. al. of $1.6 \mathrm{dyn} / \mathrm{cm}^{2}$. The increased OPN expression observed by Fisher et. al. upon increasing the flow rate from $3 \mathrm{~mL} / \mathrm{min}$ to $10 \mathrm{~mL} / \mathrm{min}$ then suggests that the biosignaling pathways responsible for osteoblastic differentiation are indeed activated at lower shear magnitudes than originally suggested.

The analysis of advective flow in the previous section was also applied to determine the effect of flow rate on Peclet number distribution within the scaffolds. The following figures illustrate the results of this analysis. 


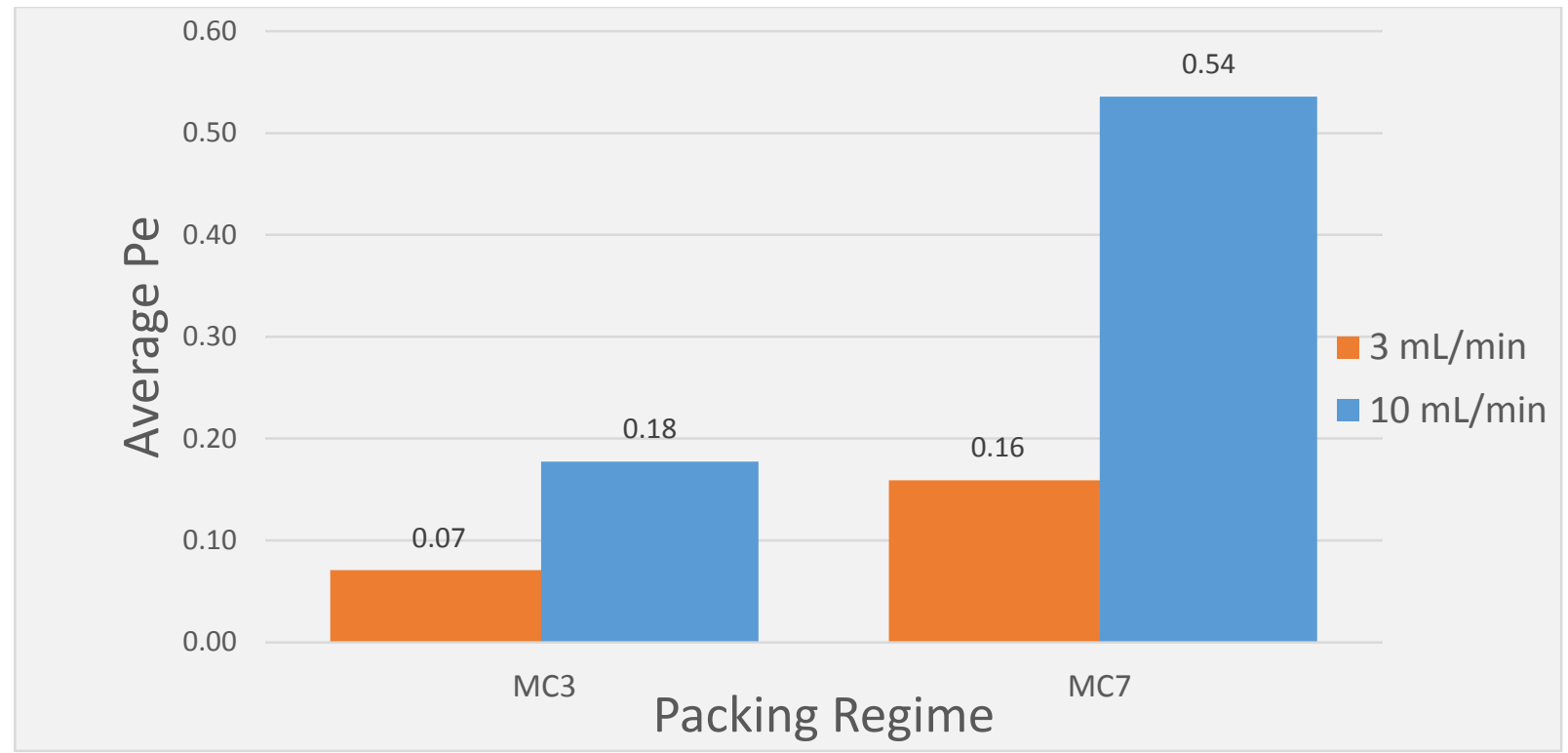

FIGURE 76 - Average Peclet number at 3 and $10 \mathrm{~mL} / \mathrm{min}$

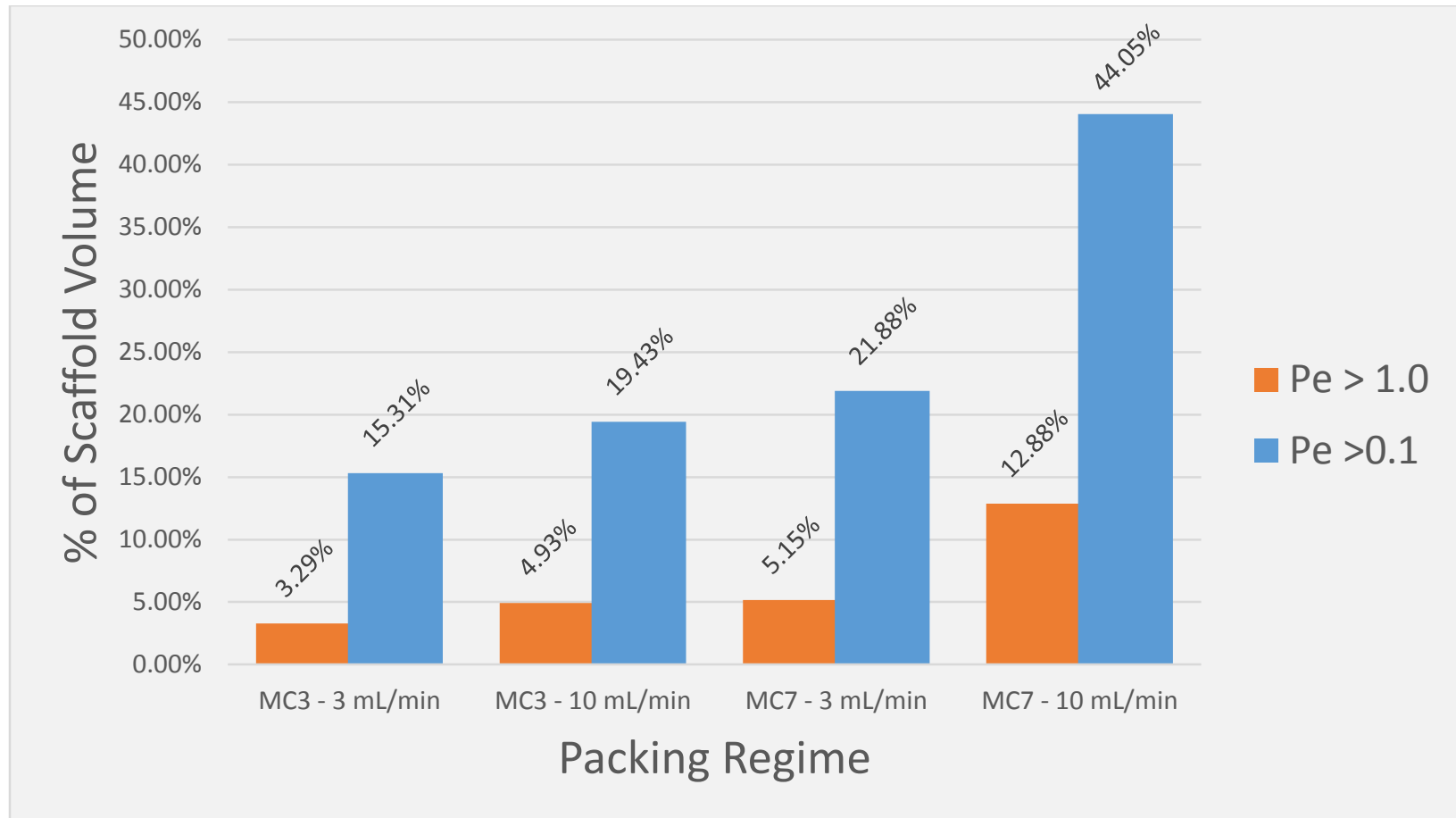

FIGURE 77 - Peclet number distributions by $\%$ of scaffold volume at 3 and $10 \mathrm{~mL} / \mathrm{min}$ 
A modest increase in advective flow within the scaffold at $10 \mathrm{~mL} / \mathrm{min}$ was observed for both packing regimes. However, a more than twofold increase was observed in MC7, with a much more modest change in MC3. Because of the presence of interior spheres in MC7, it was hypothesized that inMC7 an increase in flow rate drastically increases the amount of media flow through the interior beads. To test this hypothesis, cross sectional plots were again utilized to study penetration depth under each set of conditions.
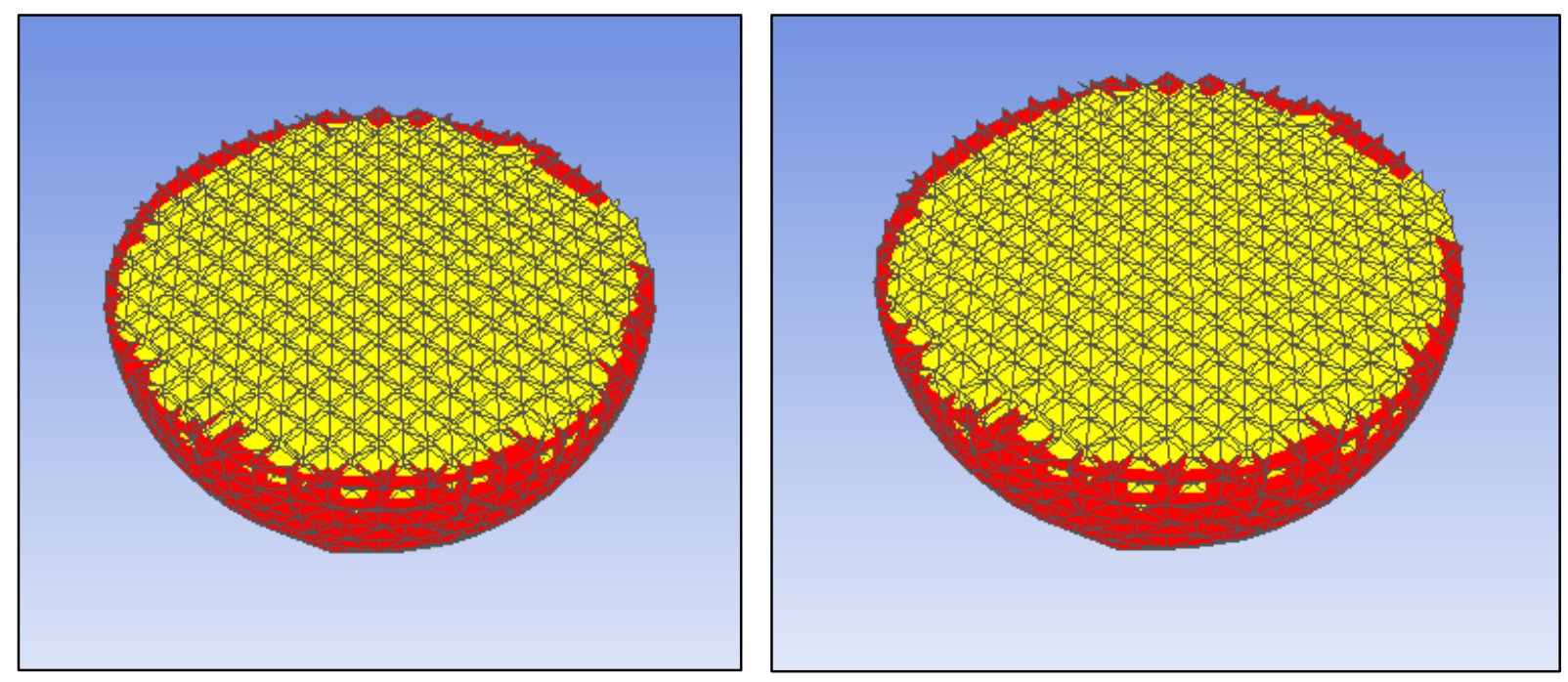

FIGURE 78 - Regions of Pe >1 in MC3 at $3 \mathrm{~mL} / \mathrm{min}$ (left) and $10 \mathrm{~mL} / \mathrm{min}$ (right) 

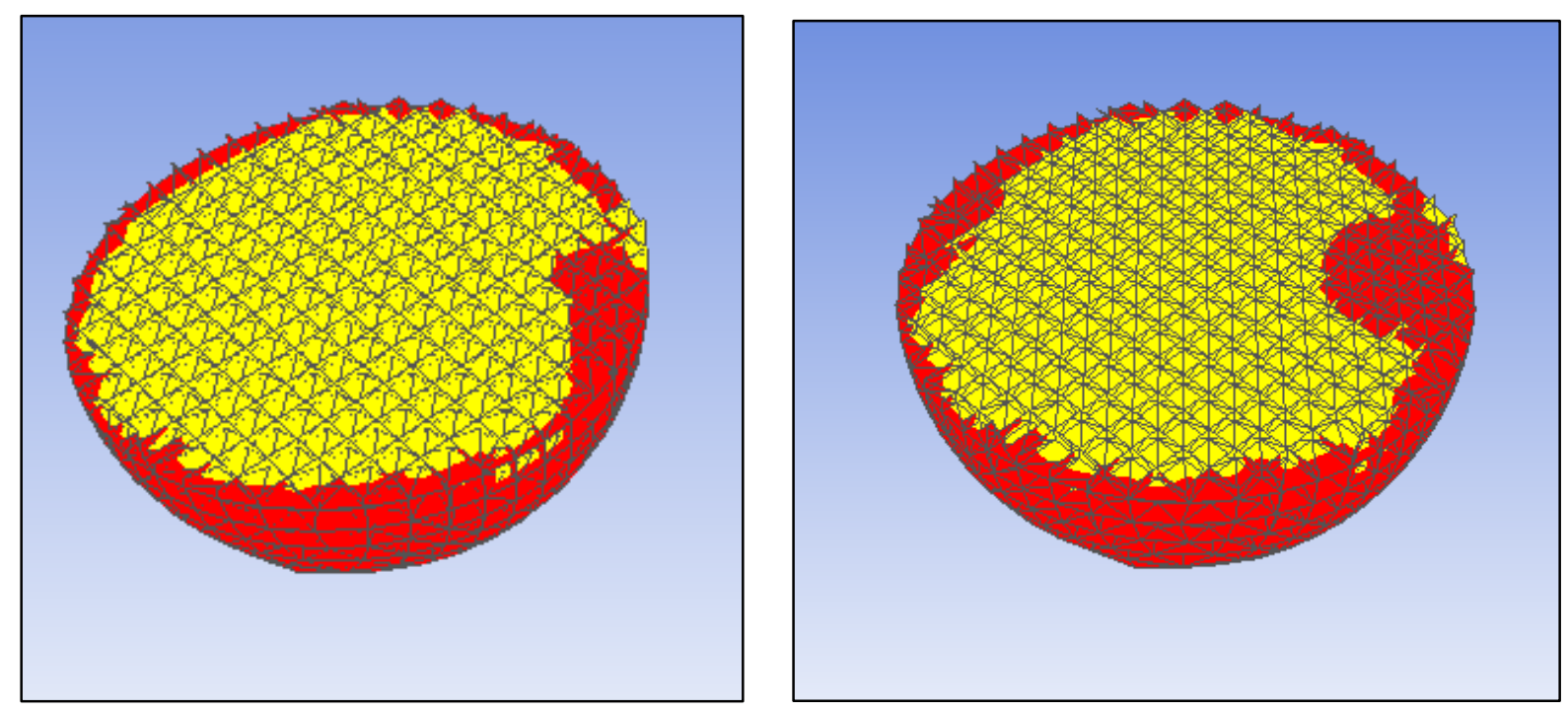

FIGURE 79 - Regions of Pe $>0.1$ in MC3 at $3 \mathrm{~mL} / \mathrm{min}$ (left) and $10 \mathrm{~mL} / \mathrm{min}$ (right)
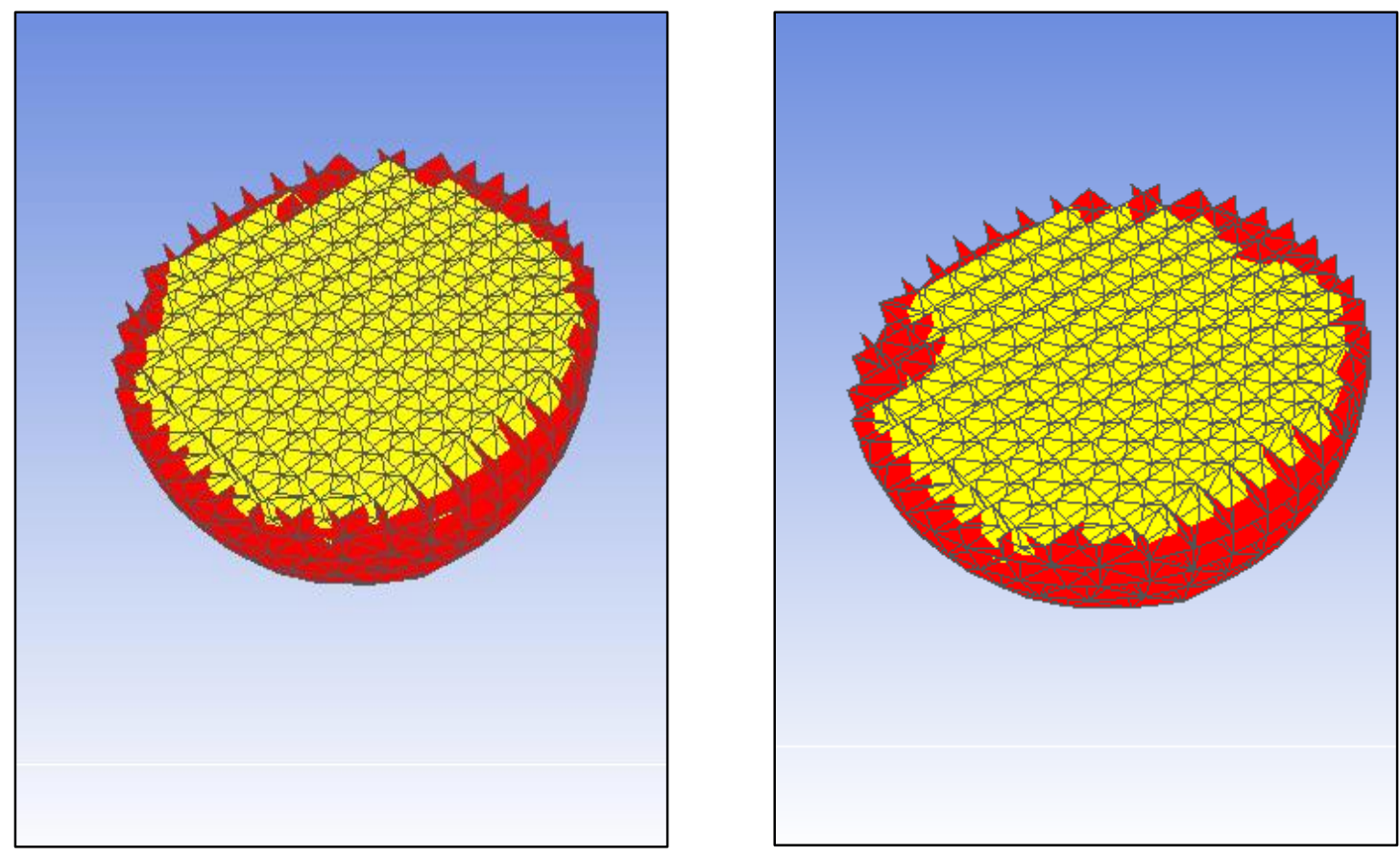

FIGURE 80 - Regions of Pe $>1$ in MC7 at $3 \mathrm{~mL} / \mathrm{min}$ (left) and $10 \mathrm{~mL} / \mathrm{min}$ (right) 

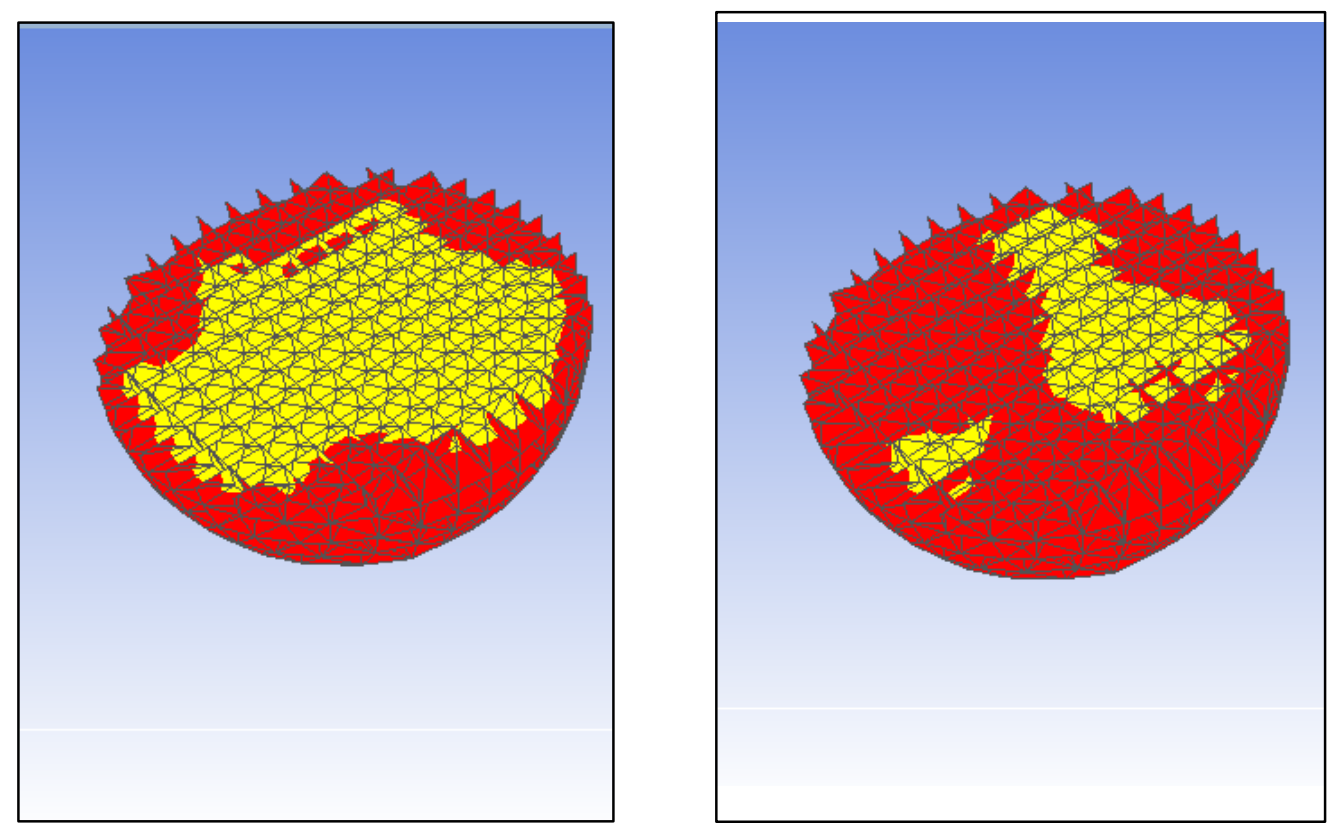

FIGURE 81 - Regions of Pe $>0.1$ in MC7 at $3 \mathrm{~mL} / \mathrm{min}$ (left) and $10 \mathrm{~mL} / \mathrm{min}$ (right)

As the contour plots illustrate, the interior beads of MC7 are affected drastically by the higher flow rate, while the beads of MC3 are affected only modestly, particularly in regions exposed to particularly high velocity bulk flow. In all cases, diffusion still serves as the predominant means of nutrient transport, particularly well beyond the surface where media penetration is low.

\section{E. Maximal Contact and Line Slip Structures}

As previously stated, line slip structures exhibit the same contact pattern as their corresponding maximal contact structure. However, because the diameter ratio is not optimized for the given packing regime, the contacts have "slipped" forming a staggered helix structure. The goal of this section was to explore the influence of selecting a diameter ratio corresponding to an ideal, maximal contact packing structure versus a non-ideal line slip structure in terms of shear stress exposure on the surface. 
Each of the following comparisons is between a line slip structure and its corresponding maximal contact structure. The goal of this comparative analysis was to determine if a compelling reason exists to select bead diameters corresponding to maximal contact structures. As before, results are presented both qualitatively and quantitatively

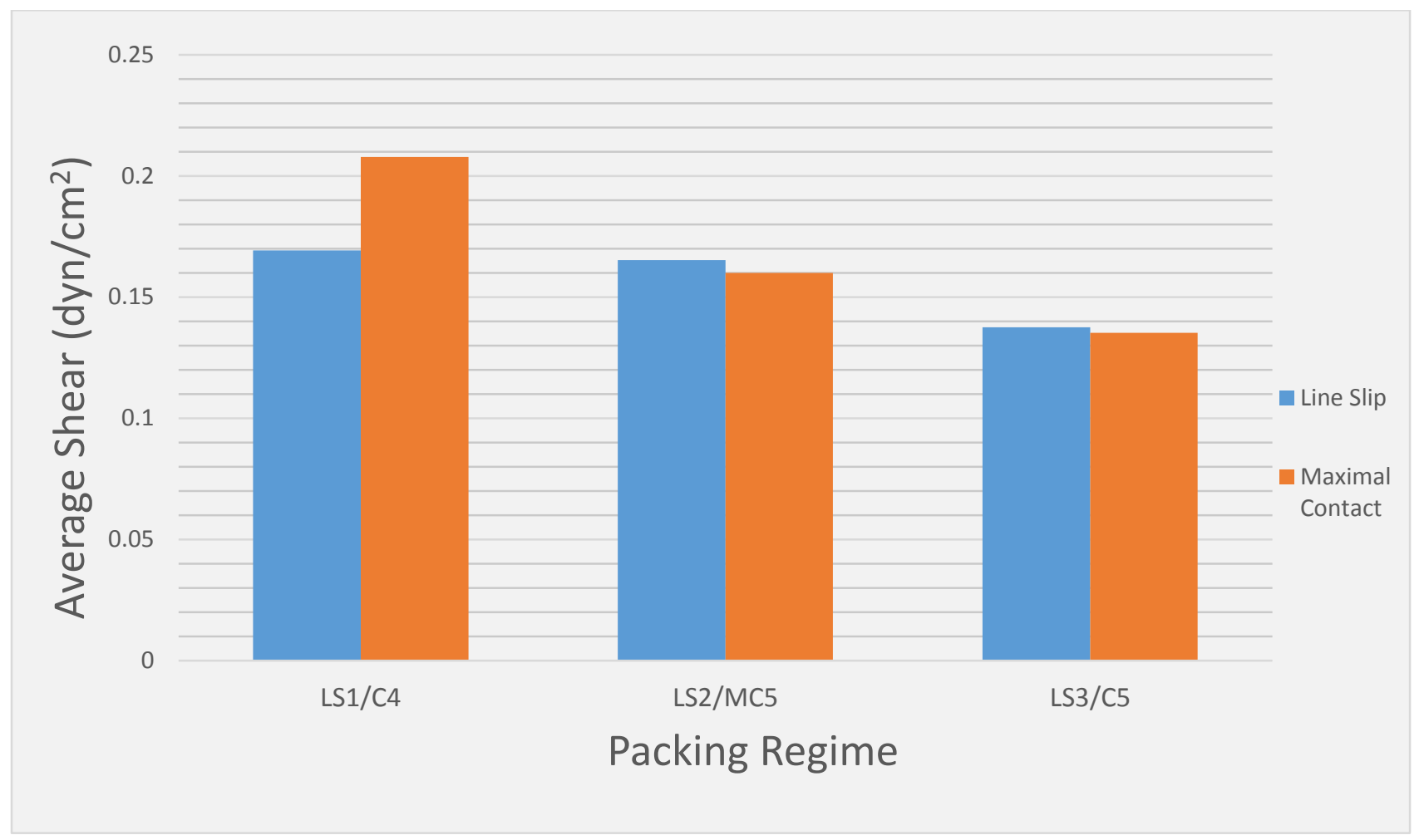

FIGURE 82 - Mean shear stress for each line slip/maximal contact pair

From the averages, only the $\mathrm{C} 4$ packing regime exhibited a significant increase in mean shear stress between the maximal contact structure and line slip structure. In the other two cases, the difference was almost negligible. This seems to suggest that the pattern of contacts between the scaffold beads has a greater effect on the mean shear stress than the void fraction of the packing. In order to better understand the similarities between the line slip and maximal contact structures, a distribution analysis was carried out as before for each pair. 


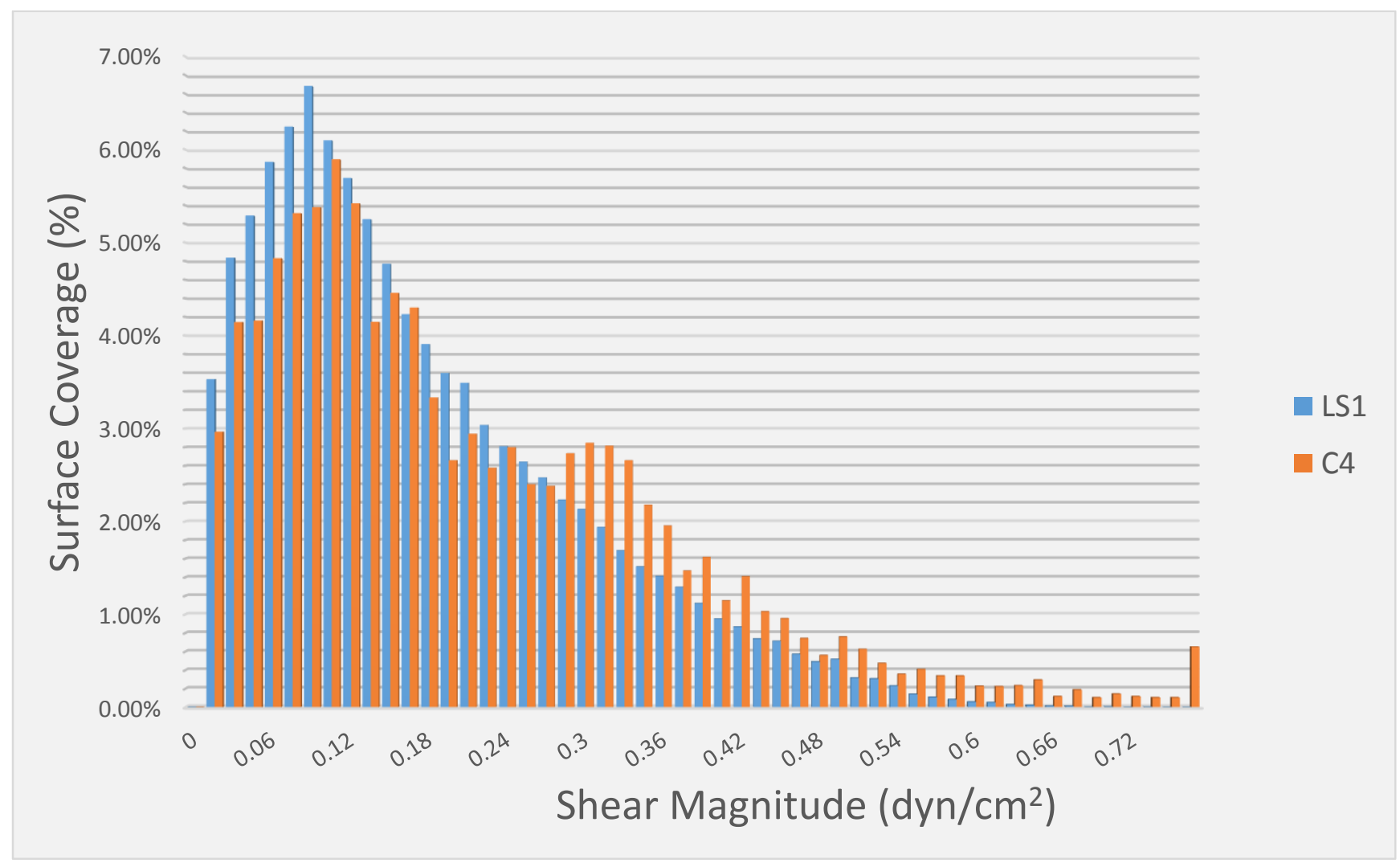

FIGURE 83 - Shear distribution for LS1 and C4 by \% of scaffold surface 

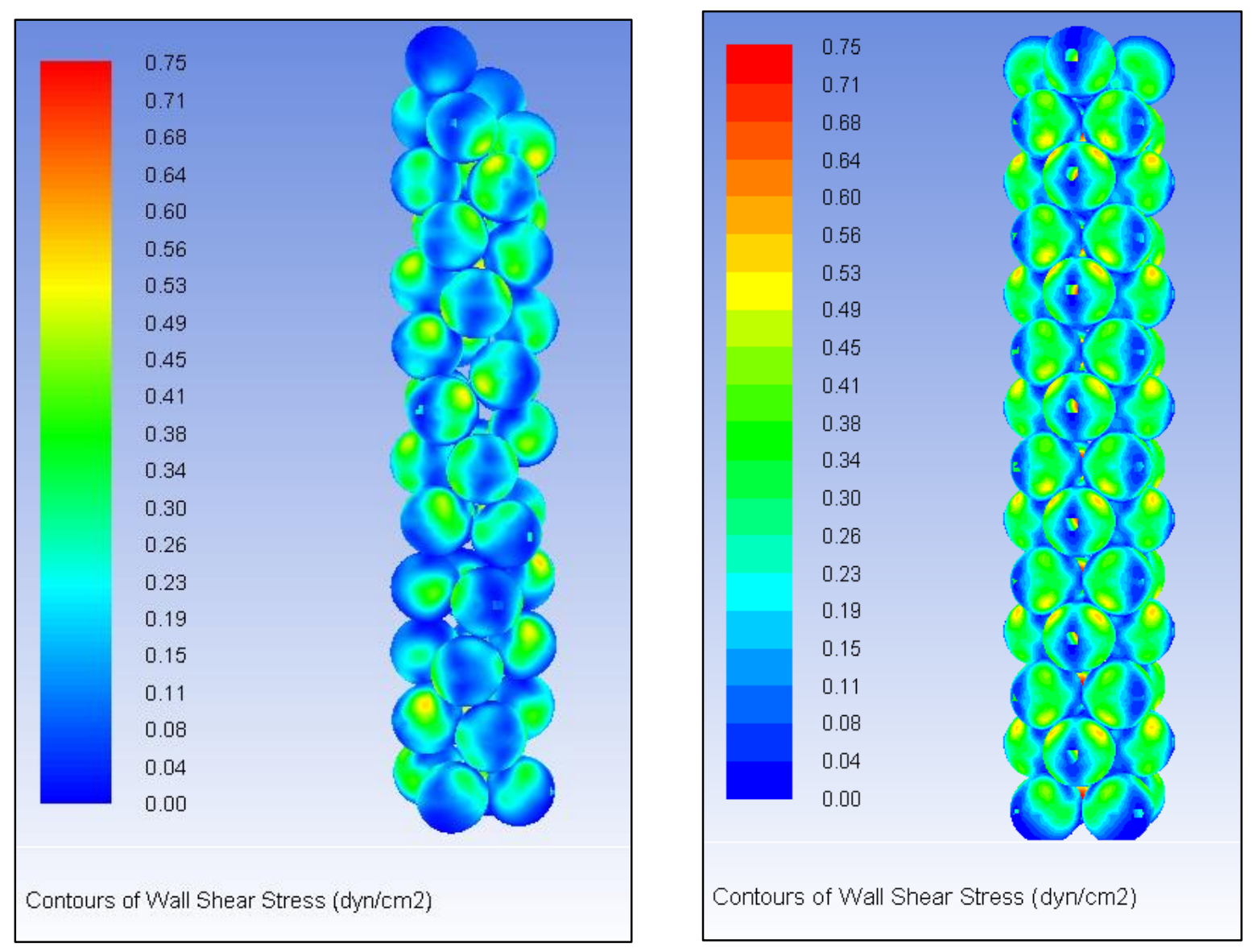

FIGURE 84 - Shear stress contour plots for LS1 (left) and C4 (right)
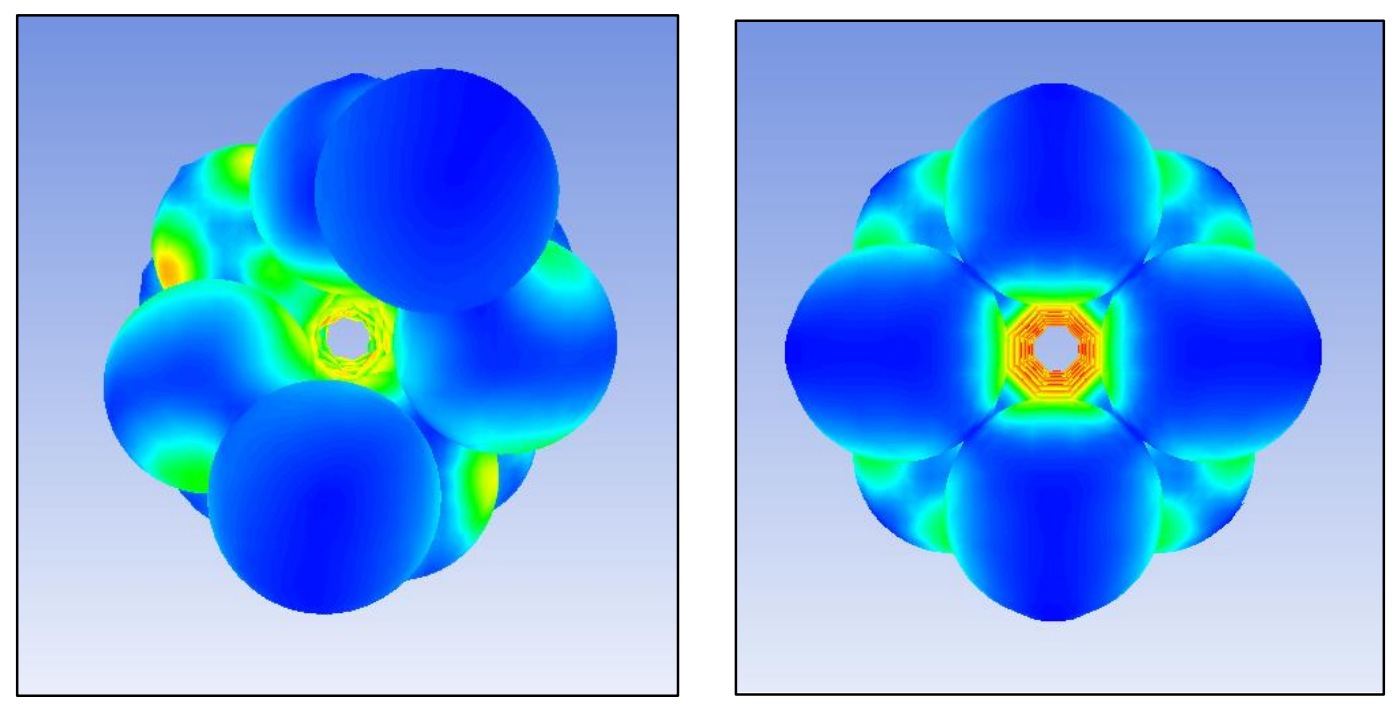

FIGURE 85 - Top-down shear stress contour plots for LS1 (left) and C4 (right) 


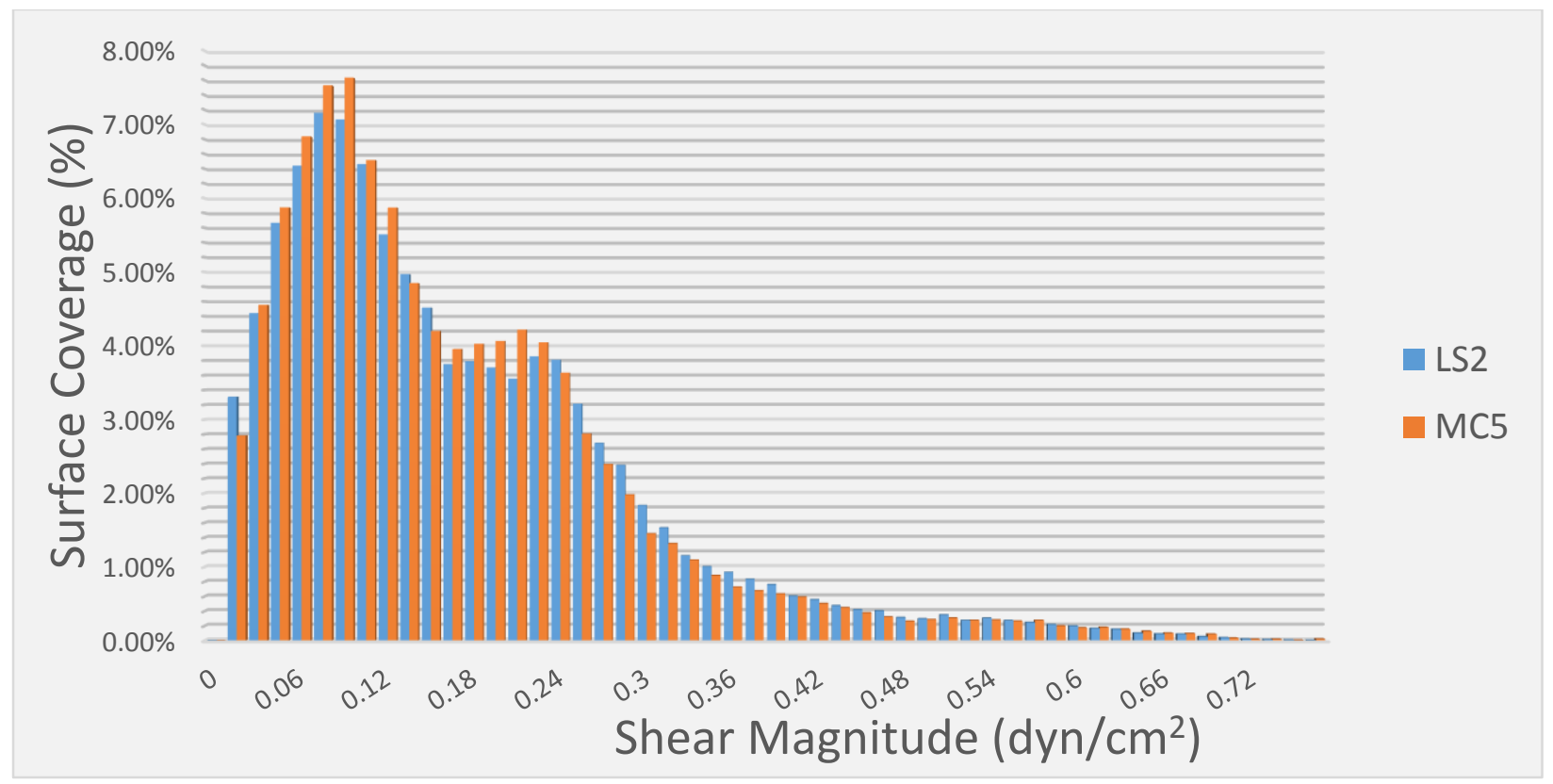

FIGURE 86 - Shear stress distribution for LS2 and MC5 by \% of surface area
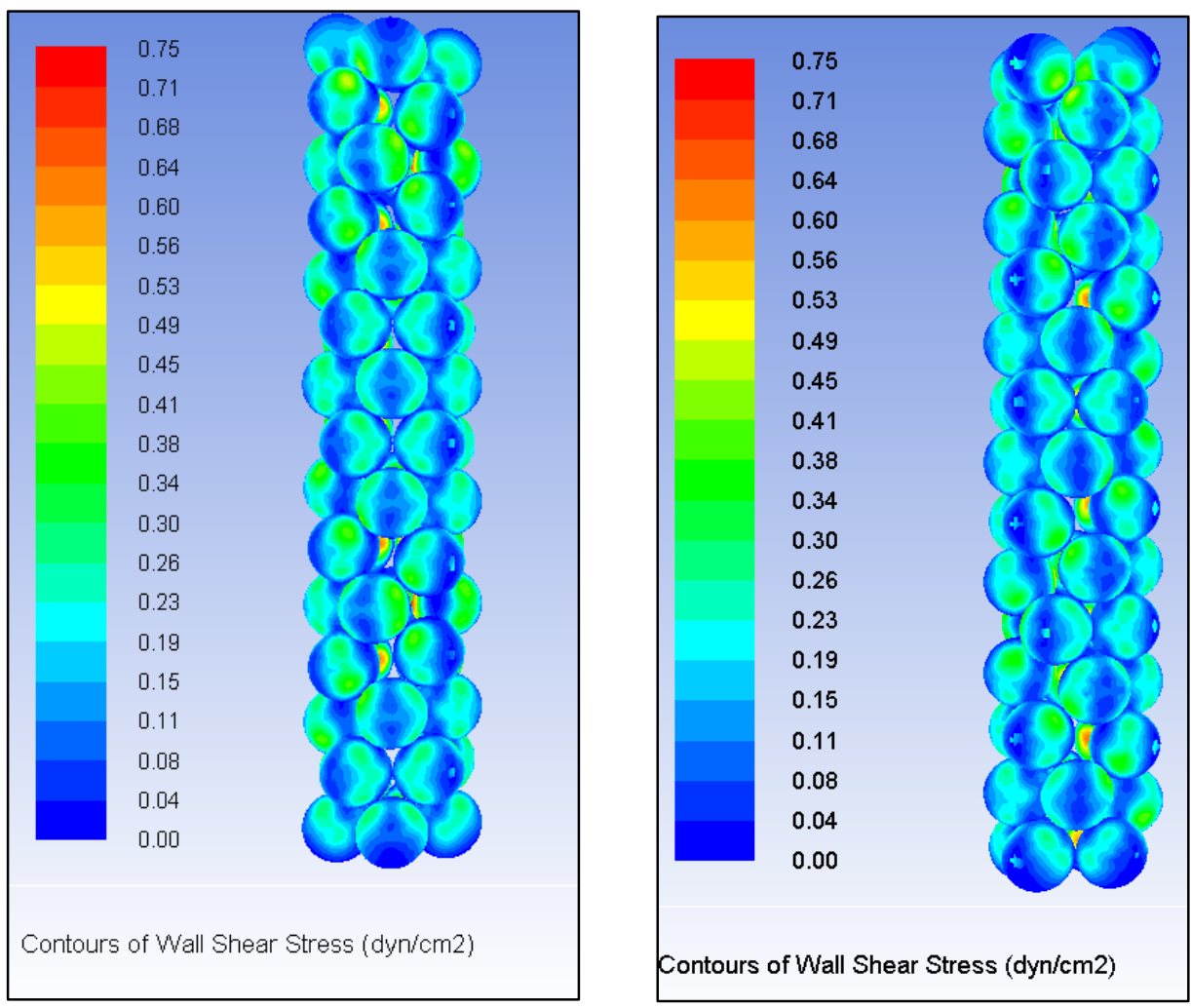

FIGURE 87 - Shear stress contour plots for LS2 (left) and MC5 (right) 

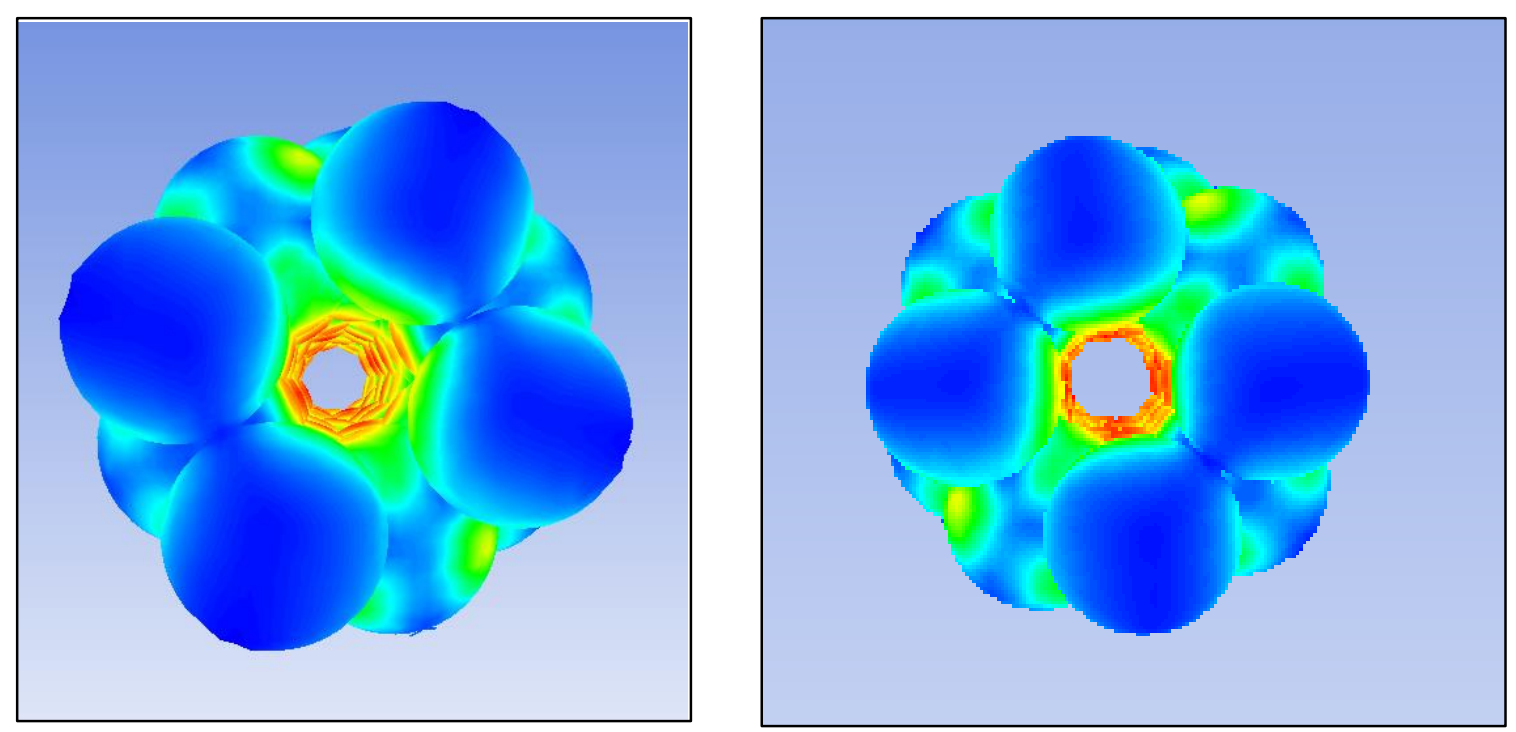

FIGURE 88 - Top down shear stress contour plots for LS2 (left) and MC5 (right)

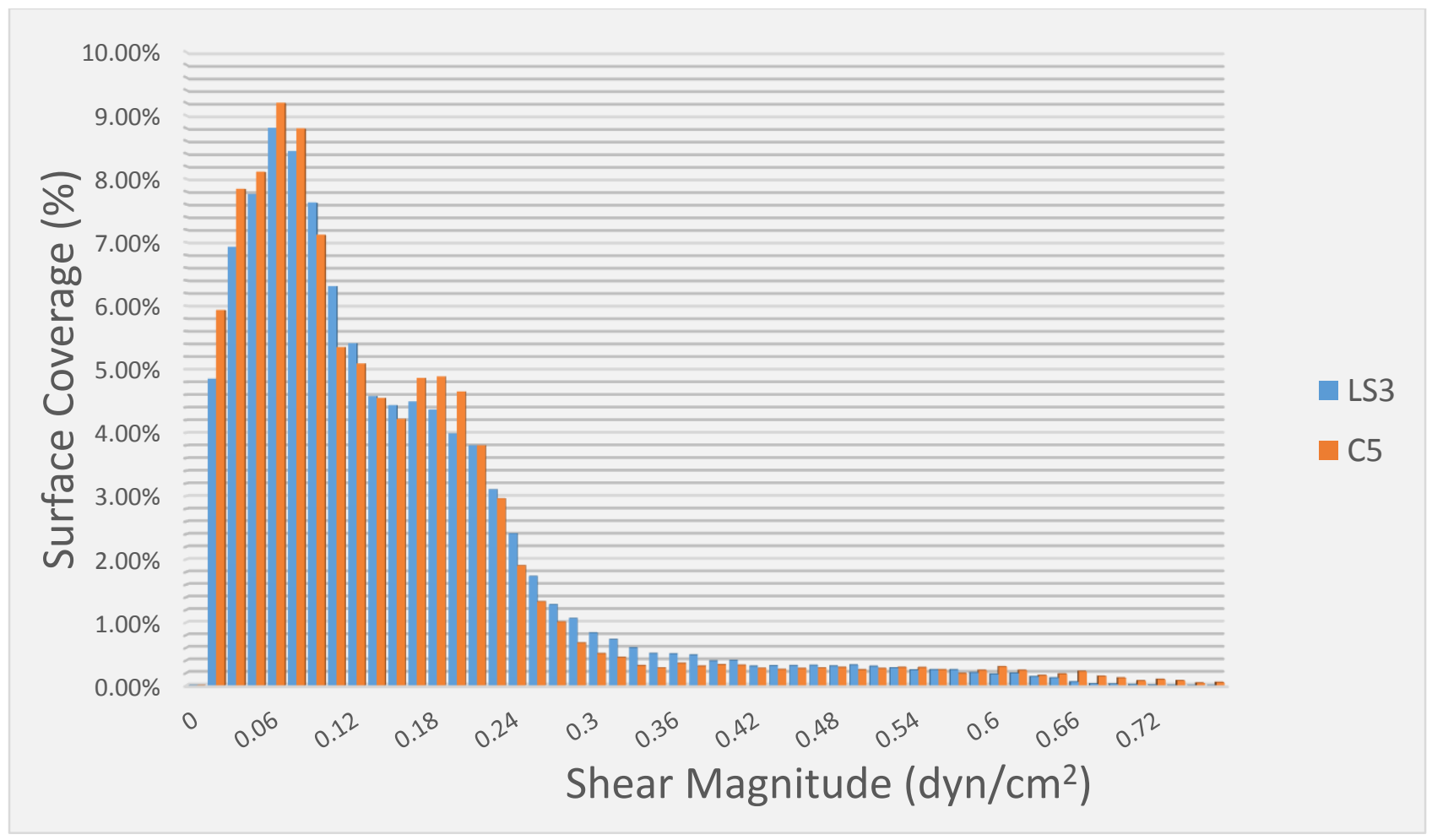

FIGURE 89 - Shear stress distribution for LS3 and C5 by \% of surface area 

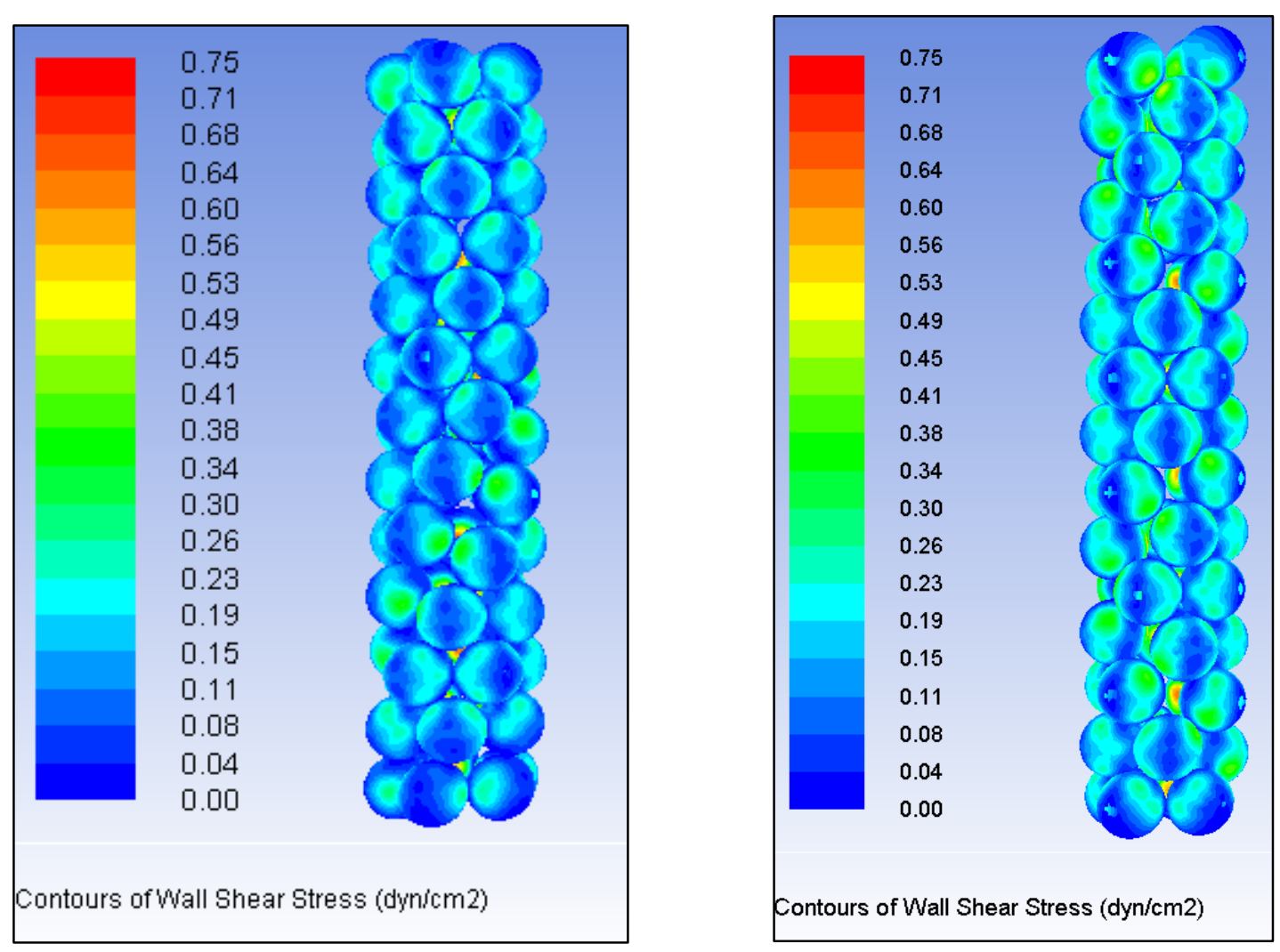

FIGURE 90 - Shear stress contour plots for LS3 (left) and C5 (right)
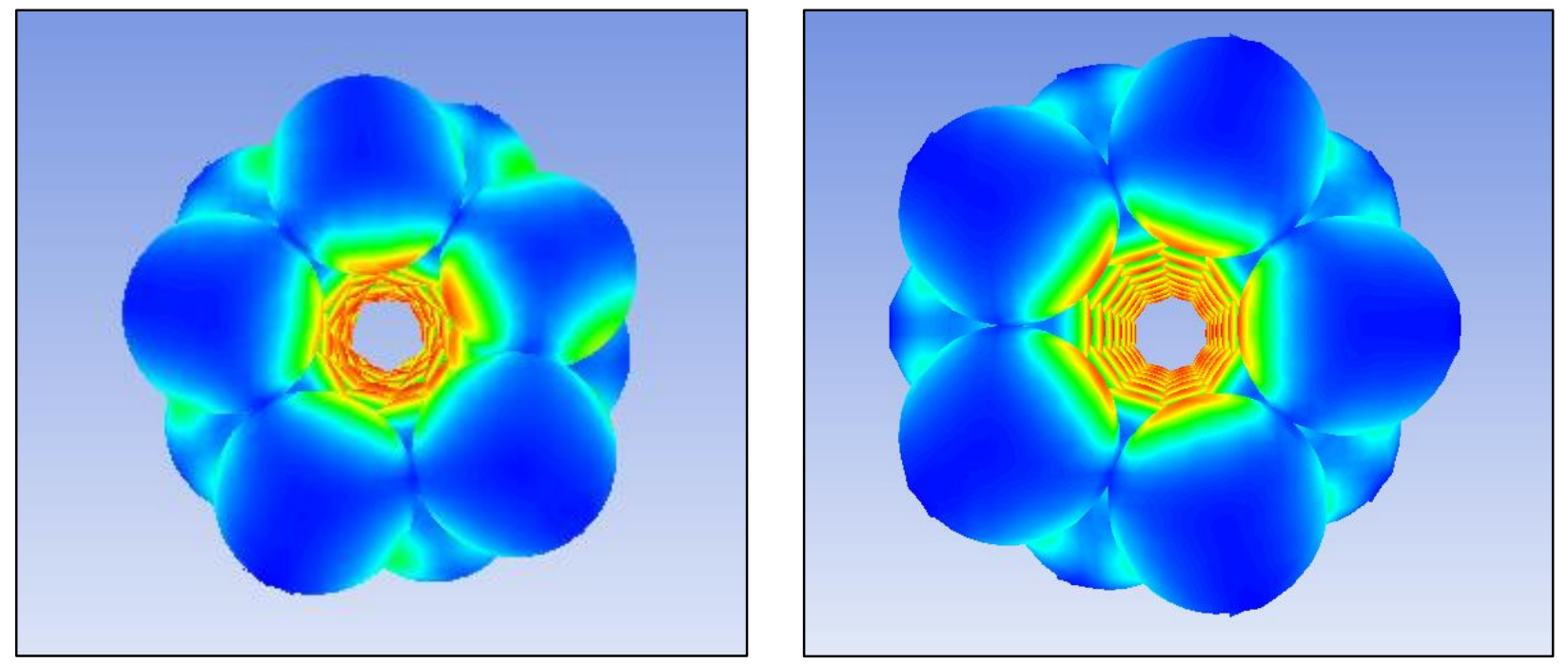

FIGURE 91 - Top down shear stress contour plots for LS3 (left) and C5 (right) 
From inspection of the previous plots, it is evident that in the LS2/MC5 and LS3/C5 pairings, the shear stress distributions are nearly identical. This suggests that although the void fractions differ and the contact points do not align exactly, there is no compelling advantage to selecting a bead diameter corresponding to the maximal contact structure. However, in the case of the LS1/C4 pairing, the "staggered helix" is more pronounced due to the larger bead diameter. This causes a more profound effect, particularly in the central channel where lower shear stresses are observed. This is evident on the distribution plot, as the LS1 distribution lacks the second peak corresponding to the shear stress located on the interior. In this case, it seems that the geometric differences between the maximal contact structure and the line slip structure convers an advantage on the maximal contact structure.

\section{F. Advective Transport in Tricalcium Phosphate Scaffolds}

In the previous sections, the extent of advective flow within the scaffolds was thoroughly characterized for alginate. The purpose of this section was to determine whether advective transport predominates in a much more permeable material, tricalcium phosphate. Fan et. al. (2013) characterized the hydraulic permeability of various TCP scaffolds. For this study, a value of 3.69/ $\mu \mathrm{m}^{2}$ was utilized. Again, MC3 and MC7 were chosen as the two packing regimes for analysis. The results are presented as before, both an average Peclet number over the entire scaffold region and a cross section showing growth media penetration into the scaffold beads. 


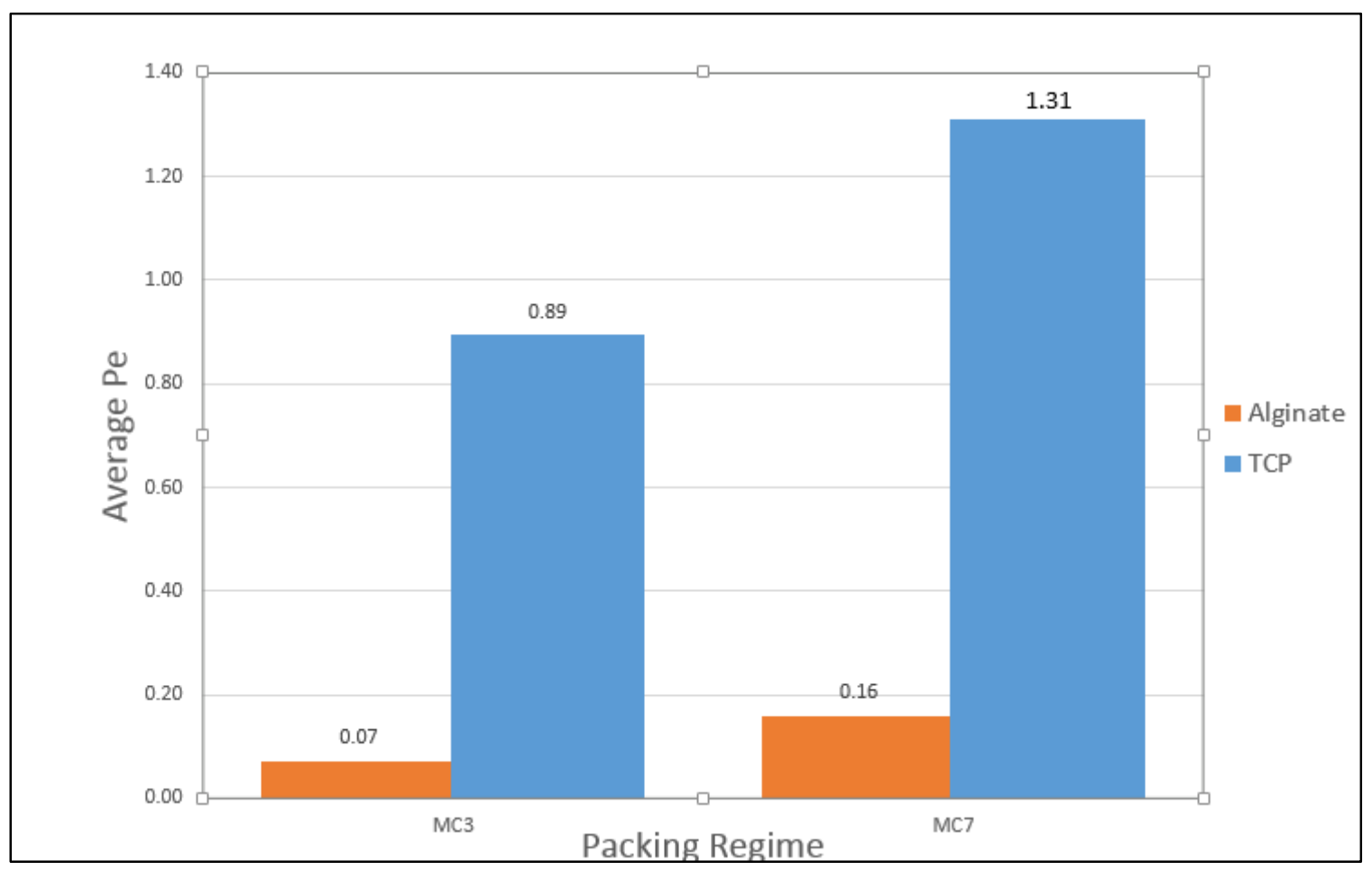

FIGURE 92 - Comparison of average Peclet number for both packing regimes and materials

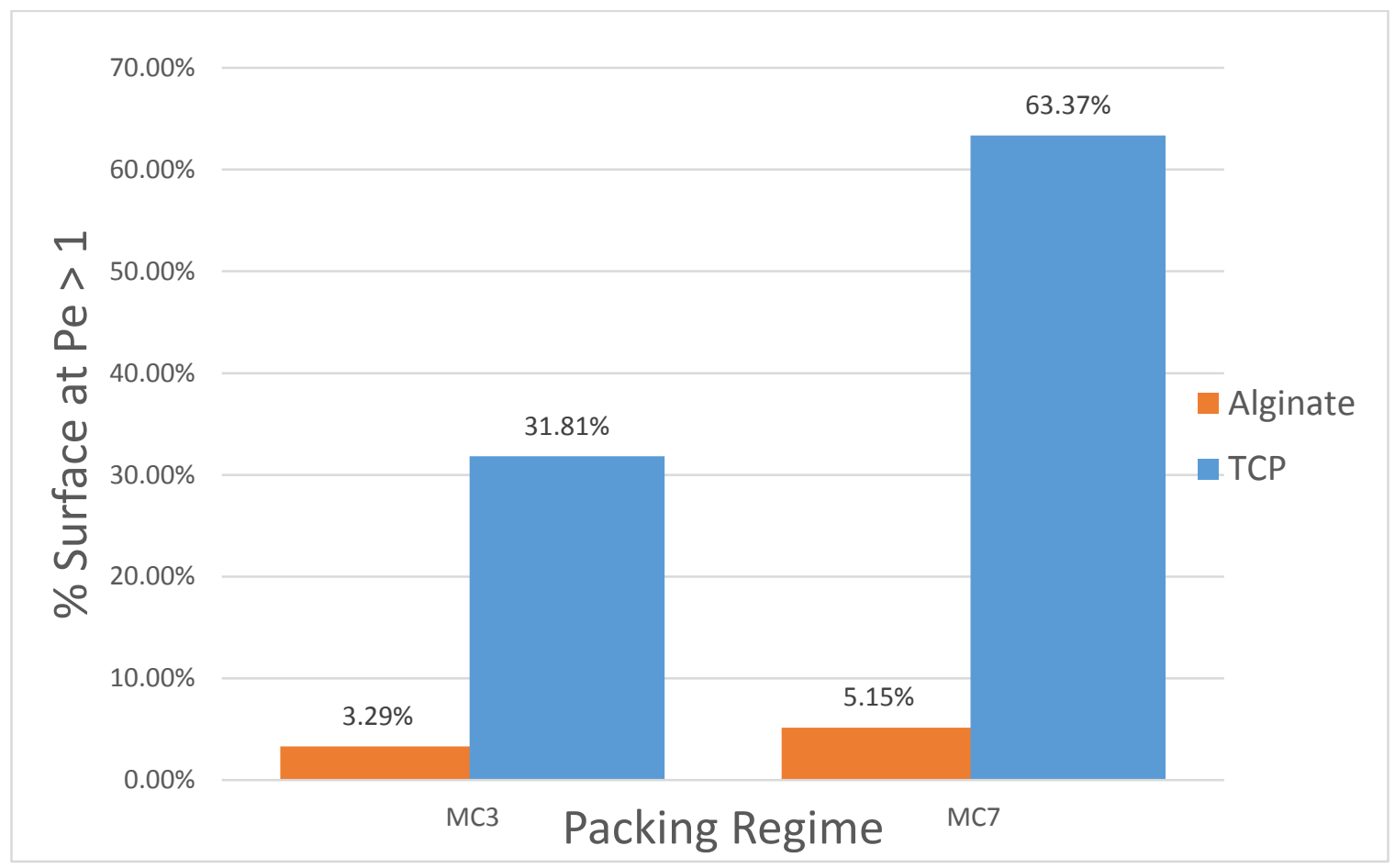

FIGURE 93 - \% of scaffold volume at Pe $>1$ 

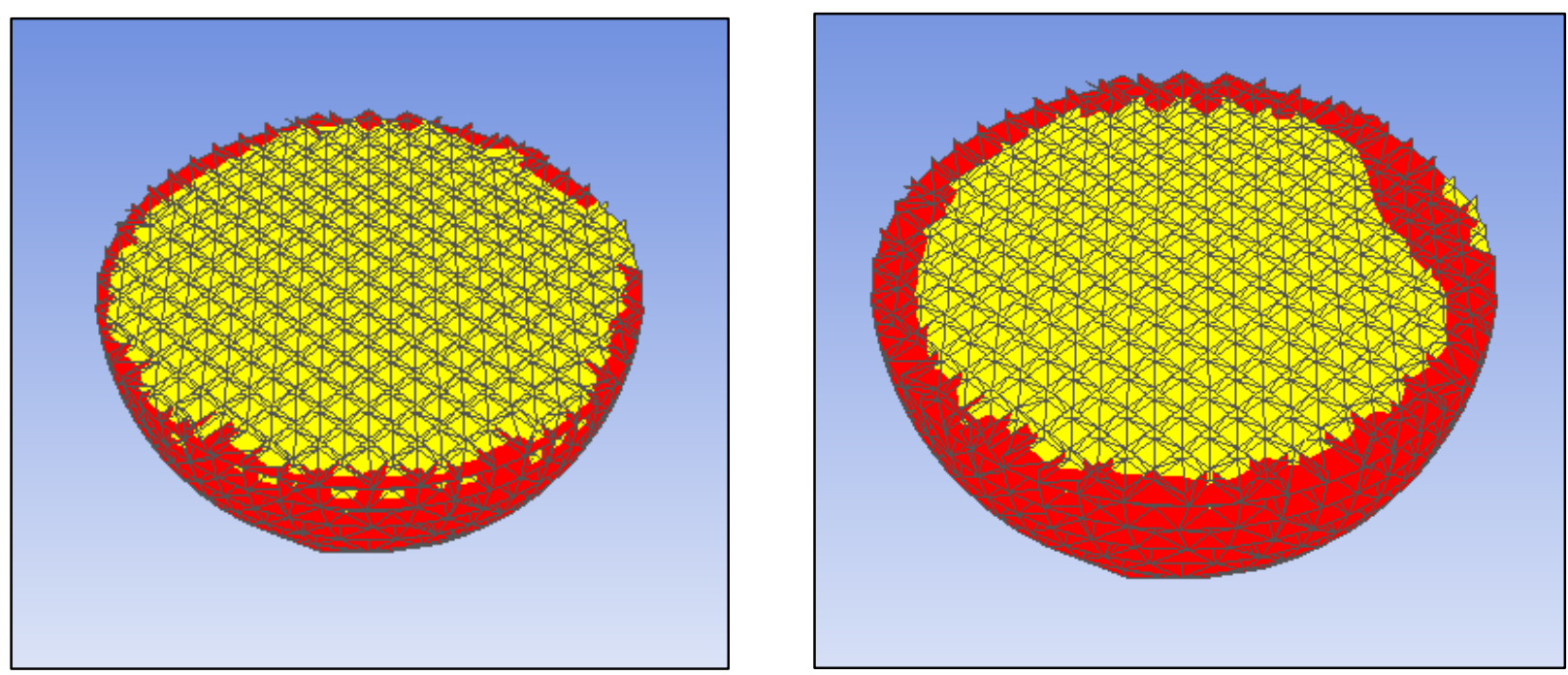

FIGURE 94 - Regions of Pe >1 on MC3 in alginate (left) and tricalcium phosphate (right)
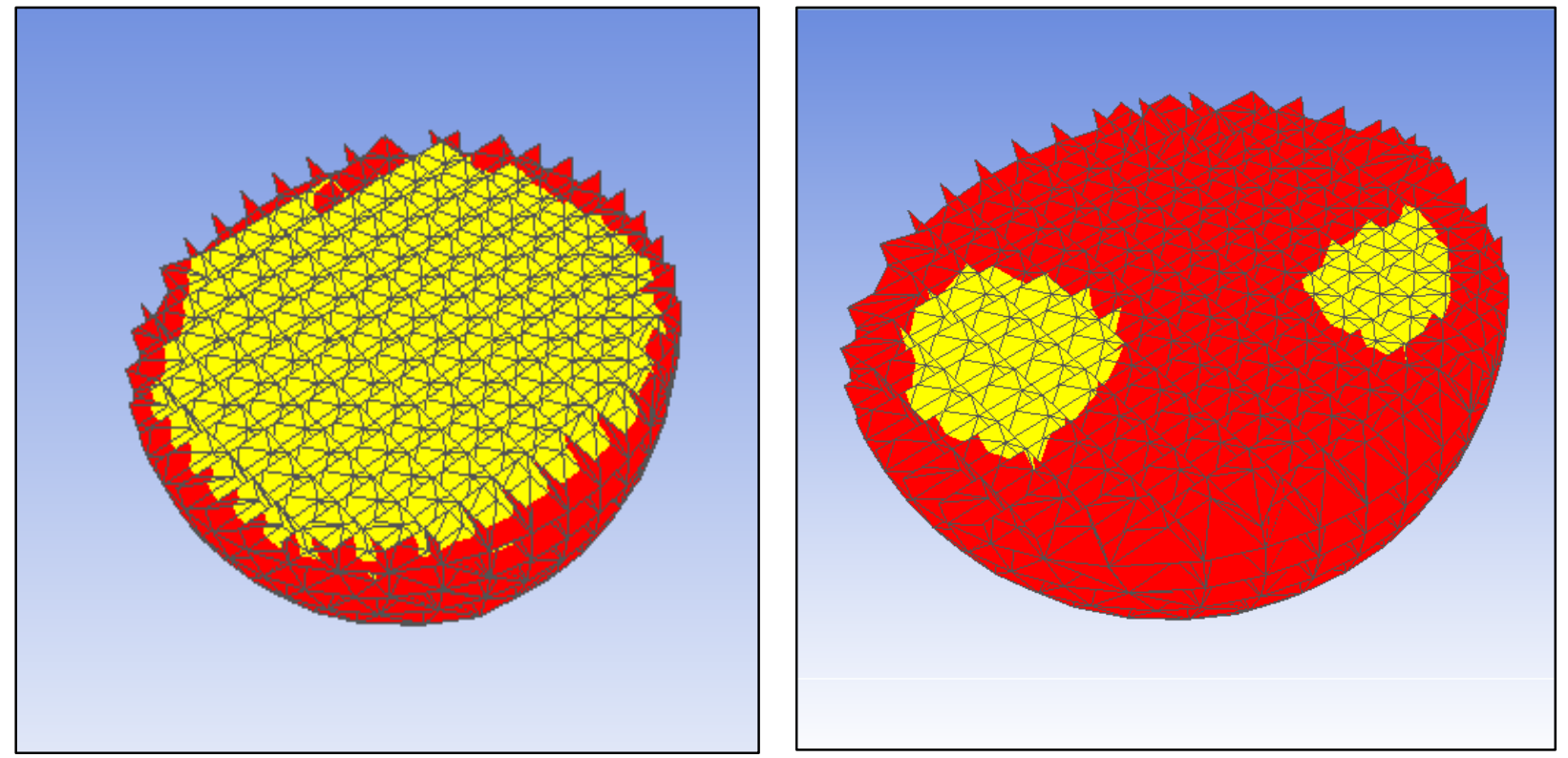

FIGURE 95 - Regions of Pe $>1$ on MC7 in alginate (left) and tricalcium phosphate (right) 
Though the use of tricalcium phosphate, a much more permeable material, enhances advective flow within the scaffold region, there still exist regions in which diffusive transport provides the majority of overall nutrient transport. This is particularly true for MC3, where the majority of the bead interior is still not penetrated by media flow. This underscores the central challenge of designing the TPS. Maintaining adequate nutrient transport to the cells at the center of each scaffold bead is important to keep cell survival high. This data gives shows that even for highly porous materials, diffusive transport is still important for carrying nutrients to the center of the scaffold. 


\section{CONCLUSION}

The first goal of this study was to identify the magnitude of shear stress and advective transport experienced in the bioreactor under normal operating conditions, using a flow rate of $3 \mathrm{~mL} / \mathrm{min}$ and simulating the permeability of alginate. Average shear stress values were found to range from 0.13-0.26 dyn $/ \mathrm{cm}^{2}$ across all cases studied. Furthermore it was determined that the distribution of shear stress on the scaffold surface was highly geometry dependent. In general, those geometries containing interior spheres exhibited a more uniform shear stress distribution, while those with a hollow central channel experienced high shear stress magnitudes directly adjacent to the channel.

After testing all possible packing regimes from $\mathrm{D} / \mathrm{d}=2.0$ to 2.82 , it was determined that the MC7 packing regime exhibited the most advantageous shear distribution, with $73.92 \%$ of the scaffold surface exhibiting a shear stress at or above $0.15 \mathrm{dyn} / \mathrm{cm}^{2}$. This level has been demonstrated by Kreke et. al. (2005) to upregulate OPN expression, a key marker for osteoblastic differentiation. This finding is logical, since the presence of interior spheres in MC7 increases the tortuosity of the fluid pathways, thus maximizing shear exposure.

The permeable media model determined that, in general, nutrient transport in alginate scaffolds predominantly occurs via diffusion, as media flow does not penetrate substantially beyond the surface of the scaffold beads. This is evidenced by average Peclet numbers on the order of 0.05-0.08. Two notable exceptions were the packing regimes $\mathrm{C} 2$ and $\mathrm{C} 4$, which experienced average Peclet numbers of 0.3 and 0.34 respectively, and a much higher degree of scaffold penetration. This is likely due to the unique geometrical arrangement of these two packing structures. In each case, the unit cells were rotated 90 degrees and translated along the axis, which created a flow pattern in which media strikes the beads perpendicular to the surface, forcing much of the flow through the porous region. 
An analysis of using an alternative flow rate of $10 \mathrm{~mL} / \mathrm{min}$ showed a great increase in average shear coverage and a modest increase of advective transport within the scaffold beads. The average Peclet number for the MC7 packing regime rose from 0.16 to 0.54 , a much more substantial increase than that for MC3. This was determined to be due to the presence of interior spheres, which do not allow a path of low resistance through which media flow can pass unimpeded. The findings of this study are consistent with experimental trials by Fisher et. al. (2011) in which enhanced OPN expression was observed upon increasing the flow rate, but lower calcification, likely resulting from an a high advective flow rate preventing cells from depositing extracellular matrix.

Prior to the study, it was hypothesized that selecting a scaffold diameter corresponding to the "maximal contact" structure for a given packing regime would result in a higher average shear stress value. This only proved to be true in the case of LS1/C4. Due to the larger beads, the disruption of the crystal structure was more apparent. C4 exhibited an average shear stress value $23.5 \%$ higher than LS1, the corresponding line slip structure.

Finally, it was determined that changing the scaffold material from alginate to tricalcium phosphate resulted in a significant increase in average Peclet number within the scaffold region. This change was again more pronounced in MC7 than MC3 due to the presence of interior spheres. $31.81 \%$ of the scaffold volume in MC3 and $63.37 \%$ of the scaffold volume in MC7 exhibited a Peclet number greater than 1, indicating a higher relative contribution from advective transport throughout much of the scaffold. However, regions toward the center of the scaffold still experienced low advective flow rate, meaning that in these regions diffusion is critical to maintain oxygen concentration 
In summary, the findings of this report provide a general estimate of the magnitude of shear exposure and advective transport in the tubular perfusion system. Given the breadth of data considered, a recommendation for which packing regime, material, or flow rate is most advantageous is not practical. Rather, this study provides the tools in order to tailor the bioreactor design to meet the needs of the study being performed, and to better understand phenomena which are observed in the bioreactor and how they relate to fluid dynamics. In general, however, the selection of packing regimes containing interior spheres seems to confer some advantages. The influence of packing regime and the presence of interior spheres was not considered prior to this study. 


\section{RECOMMENDATIONS}

Various improvements can be made to the TPS model through future studies. While all of the cases studied were in the laminar or low transition flow regime, and thus modeled as laminar, future studies on high flow rates will need to include a turbulence model. Additionally, a species diffusion model for oxygen could be integrated such that oxygen concentration could be monitored as a function of time across the scaffold beads. By including reaction terms, cellular respiration could be simulated. This would give an excellent indication of cell survivability. 


\section{REFERENCES CITED}

Anisi, F., Salehi-Nik, N., Amoabediny, G., Pouran, B., Haghighipour, N., \& Zandieh-Doulabi, B. (2014). Applying shear stress to endothelial cells in a new perfusion chamber: hydrodynamic analysis. Journal of Artificial Organs, 17(4), 329-336. doi: 10.1007/s10047014-0790-0

Augst, A. K., H.J.; Mooney, D. (2006). Alginate Hydrogels as Biomaterials Macromolecular Bioscience, 6, 623-633.

Baker, M. (2011). CFD Simulation of Flow Through Packed Beds Using the Finite Volume Technique. (Doctor of Philosophy), University of Exeter, Exeter, Devon, UK.

Bird, R. B., Stewart, W.E., Lightfoot, E.N. (2006). Transport Phenomena: John Wiley \& Sons.

De Boodt, S. O., H.; Berckmans, D.; Schrooten, J. . (2010). Bi-Modular Flow Characterization in Tissue Engineering Scaffolds Using Computational Fluid Dynamics and Particle Imaging Velocimetry. Tissue Engineering Part C-Methods, 16(6), 1553-1563.

Duscha, H. H., Mary; Sparrell, Courtney. (2012). Computational Fluid Dynamics Analysis of TwoPhase Flow in a Packed Bed Reactor. (Master of Science), Worcester Polytechnic Institute.

Eiselt, P. Y., Julia; Latvala, Rachel; Shea, Lonnie; Mooney, David. (2000). Porous Carriers for Biomedical Applications Based on Alginate Hydrogels. Biomaterials, 21.

Fan, J. J., Xiaoling; Huang, Yan; Fu, Bingmei; Fan, Yubo. (2013). Greater Scaffold Permeability Promotes Growth of Osteoblastic Cells in a Perfused Bioreactor. Journal of Tissue Engineering and Regenerative Medicine.

He, J. L., Dichen; Liu, Yaxiong; Li, Xiao; Xu, Shanlong; Li, Bingsheng. (2011). Computational Fluid Dynamics for Tissue Engineering Applications. Journal of Mechanics in Medicine and Biology, 11(2), 307-319. 
Huysmans, M. D., Alain. (2003). Review of the use of Peclet numbers to determine the relative improtance of advection and diffusion in low permeability environements. Hydrogeology Journal, 10.

Hwang, C. M., Sant, S., Masaeli, M., Kachouie, N. N., Zamanian, B., Lee, S. H., \& Khademhosseini, A. (2010). Fabrication of three-dimensional porous cell-laden hydrogel for tissue engineering. Biofabrication, 2(3), 035003. doi: 10.1088/1758-5082/2/3/035003

Johnson, E. M., Berk, D. A., Jain, R. K., \& Deen, W. M. (1996). Hindered diffusion in agarose gels: test of effective medium model. Biophysical Journal, 70(2), 1017-1023. doi: 10.1016/S0006-3495(96)79645-5

Kreke, M. R., Huckle, W. R., \& Goldstein, A. S. (2005). Fluid flow stimulates expression of osteopontin and bone sialoprotein by bone marrow stromal cells in a temporally dependent manner. Bone, 36(6), 1047-1055. doi: 10.1016/j.bone.2005.03.008

Kreke, M. R., Sharp, L. A., Lee, Y. W., \& Goldstein, A. S. (2008). Effect of intermittent shear stress on mechanotransductive signaling and osteoblastic differentiation of bone marrow stromal cells. Tissue Eng Part A, 14(4), 529-537. doi: 10.1089/tea.2007.0068

Lawrence, B. J., Devarapalli, M., \& Madihally, S. V. (2009). Flow dynamics in bioreactors containing tissue engineering scaffolds. Biotechnology and Bioengineering, 102(3), 935947. doi: 10.1002/bit.22106

Lesman, A., Blinder, Y., \& Levenberg, S. (2010). Modeling of flow-induced shear stress applied on 3D cellular scaffolds: Implications for vascular tissue engineering. Biotechnology and Bioengineering, 105(3), 645-654. doi: 10.1002/bit.22555

Li, D. Q., Tang, T. T., Lu, J. X., \& Dai, K. R. (2009). Effects of Flow Shear Stress and Mass Transport on the Construction of a Large-Scale Tissue-Engineered Bone in a Perfusion Bioreactor. Tissue Engineering Part A, 15(10), 2773-2783. doi: 10.1089/ten.tea.2008.0540

Ma, C. Y. J., Kumar, R., Xu, X. Y., \& Mantalaris, A. (2007). A combined fluid dynamics, mass transport and cell growth model for a three-dimensional perfused biorector for tissue engineering of haematopoietic cells. Biochemical Engineering Journal, 35(1), 1-11. doi: 10.1016/j.bej.2006.11.024 
Mughal, A. C., H.K.; Weaire D.; Hutzler, S. (2012). Dense Packing of Spheres Inside Cylinders: Simulations. Physical Review, 85.

Papantoniou, I., Guyot, Y., Sonnaert, M., Kerckhofs, G., Luyten, F. P., Geris, L., \& Schrooten, J. (2014). Spatial Optimization in Perfusion Bioreactors Improves Bone Tissue-Engineered Construct Quality Attributes. Biotechnology and Bioengineering, 111(12), 2560-2570. doi: 10.1002/bit.25303

Patrachari, A. P., Jagdeep; Madihally; Madihally, Sundrarajan. (2012). Application of Computational Fluid Dynamics in Tissue Engineering Journal of Bioscience and Bioengineering, 114(2), 123-131.

Sikavitsas, V. I., Temenoff, J. S., \& Mikos, A. G. (2001). Biomaterials and bone mechanotransduction. Biomaterials, 22(19), 2581-2593.

Truscello, S., de Boodt, S., Leroy, T., Schrooten, J., Berckmans, D., \& Van Oosterwyck, H. (2008). Fluid flow within bone tissue engineering scaffolds: a computational and experimental study. Journal of Biomechanics, 41(suppl1), S83. doi: 10.1016/S0021-9290(08)70083-6

Truscello, S. S., Jan; Oosterwyck, Hans. (2011). A Computational Tool for the Upscaling of Regular Scaffolds During In Vitro Perfusion Culture. Tissue Engineering Part C-Methods, 17(6), 619-630.

Yeatts, A. B., Choquette, D. T., \& Fisher, J. P. (2013). Bioreactors to influence stem cell fate: Augmentation of mesenchymal stem cell signaling pathways via dynamic culture systems. Biochimica Et Biophysica Acta-General Subjects, 1830(2), 2470-2480. doi: 10.1016/j.bbagen.2012.06.007

Yeatts, A. B., \& Fisher, J. P. (2011). Bone tissue engineering bioreactors: Dynamic culture and the influence of shear stress. Bone, 48(2), 171-181. doi: 10.1016/j.bone.2010.09.138

Yeatts, A. B., \& Fisher, J. P. (2011). Tubular Perfusion System for the Long-Term Dynamic Culture of Human Mesenchymal Stem Cells. Tissue Engineering Part C-Methods, 17(3), 337-348. doi: 10.1089/ten.tec.2010.0172 
Yeatts, A. B., Geibel, E. M., Fears, F. F., \& Fisher, J. P. (2012). Human mesenchymal stem cell position within scaffolds influences cell fate during dynamic culture. Biotechnology and Bioengineering, 109(9), 2381-2391. doi: 10.1002/bit.24497

Yeatts, A. B., Gordon, C. N., \& Fisher, J. P. (2011). Formation of an Aggregated Alginate Construct in a Tubular Perfusion System. Tissue Engineering Part C-Methods, 17(12), 1171-1178. doi: 10.1089/ten.tec.2011.0263 


\section{APPENDIX}

TABLE II - CALCULATION OF VOID FRACTIONS

\begin{tabular}{|c|c|c|c|c|c|c|c|c|}
\hline Packing Regime & $D / d$ & $\mathrm{~d}(\mathrm{~mm})$ & Height (mm) & Total Vol. $\left(\mathrm{mm}^{3}\right)$ & Truncated Vol. $\left(\mathrm{mm}^{3}\right)$ & $\phi($ Tot) & $\phi(\mathrm{Tr})$ & $\phi($ Expected) \\
\hline $\mathrm{C} 2$ & 2.0000 & 3.1750 & 31.77 & 469.23 & 331.49 & 0.466370664 & 0.480811305 & 0.47 \\
\hline MC1 & 2.0390 & 3.1143 & 29.4 & 411.2 & 284.41 & 0.441640043 & 0.462919847 & 0.47 \\
\hline $\mathrm{C} 3$ & 2.1547 & 2.9470 & 31.7 & 524.77 & 364.7 & 0.522723958 & 0.530687304 & 0.53 \\
\hline MC2 & 2.2247 & 2.8543 & 29.87 & 462.68 & 325.92 & 0.489111836 & 0.517935659 & 0.54 \\
\hline MC3 & 2.2905 & 2.7723 & 31.12 & 503.12 & 359.85 & 0.510498725 & 0.538009893 & 0.54 \\
\hline LS1 & 2.3800 & 2.6681 & 30.25 & 447.47 & 325.4 & 0.467090692 & 0.507405522 & 0.52 \\
\hline $\mathrm{C4}$ & 2.4142 & 2.6303 & 30.03 & 533.52 & 364.22 & 0.560993773 & 0.574176626 & 0.54 \\
\hline MC4 & 2.4863 & 2.5540 & 29.98 & 488.48 & 334.97 & 0.514491053 & 0.529386787 & 0.54 \\
\hline LS2 & 2.5440 & 2.4961 & 30.25 & 488.52 & 338.24 & 0.509940655 & 0.527427301 & 0.53 \\
\hline MC5 & 2.5712 & 2.4697 & 30.31 & 473.24 & 328.21 & 0.49301278 & 0.510275308 & 0.53 \\
\hline LS3 & 2.6550 & 2.3917 & 28.95 & 465.8 & 317.34 & 0.508058339 & 0.528783958 & 0.52 \\
\hline $\mathrm{C} 5$ & 2.7013 & 2.3507 & 27.7 & 476.09 & 309.32 & 0.542715167 & 0.551819957 & 0.54 \\
\hline MC6 & 2.7306 & 2.3255 & 26.55 & 427.74 & 274.32 & 0.508719058 & 0.523386049 & 0.54 \\
\hline MC7 & 2.8211 & 2.2509 & 29.51 & 489.64 & 335.88 & 0.523926479 & 0.543612636 & 0.55 \\
\hline
\end{tabular}

TABLE III - CALCULATION OF REYNOLDS NUMBERS

\begin{tabular}{|c|c|c|c|c|c|c|}
\hline Packing Regime & Flow Rate & $\varepsilon$ (Void) & $\rho(\mathrm{kg} / \mathrm{m} 3)$ & $v(m / s)$ & $\underset{\left(\mathrm{kg} / \mathrm{m}^{*} \mathrm{~s}\right)}{\mu}$ & $\operatorname{Re}$ \\
\hline $\mathrm{C} 2$ & $3 \mathrm{~mL} / \mathrm{min}$ & 0.533629336 & 993 & 0.0016 & 0.00078 & 13.86714747 \\
\hline MC1 & $3 \mathrm{~mL} / \mathrm{min}$ & 0.558359957 & 993 & 0.0016 & 0.00078 & 14.36357956 \\
\hline $\mathrm{C} 3$ & $3 \mathrm{~mL} / \mathrm{min}$ & 0.477276042 & 993 & 0.0016 & 0.00078 & 11.48389299 \\
\hline MC2 & $3 \mathrm{~mL} / \mathrm{min}$ & 0.510888164 & 993 & 0.0016 & 0.00078 & 11.88690321 \\
\hline MC3 & $3 \mathrm{~mL} / \mathrm{min}$ & 0.489501275 & 993 & 0.0016 & 0.00078 & 11.06173872 \\
\hline LS1 & $3 \mathrm{~mL} / \mathrm{min}$ & 0.532909308 & 993 & 0.0016 & 0.00078 & 11.6351017 \\
\hline $\mathrm{C} 4$ & $3 \mathrm{~mL} / \mathrm{min}$ & 0.439006227 & 993 & 0.0016 & 0.00078 & 9.550301183 \\
\hline MC4 & $3 \mathrm{~mL} / \mathrm{min}$ & 0.485508947 & 993 & 0.0016 & 0.00078 & 10.11153285 \\
\hline LS2 & $3 \mathrm{~mL} / \mathrm{min}$ & 0.490059345 & 993 & 0.0016 & 0.00078 & 9.970377668 \\
\hline MC5 & $3 \mathrm{~mL} / \mathrm{min}$ & 0.50698722 & 993 & 0.0016 & 0.00078 & 10.20362095 \\
\hline LS3 & $3 \mathrm{~mL} / \mathrm{min}$ & 0.491941661 & 993 & 0.0016 & 0.00078 & 9.588932117 \\
\hline $\mathrm{C} 5$ & $3 \mathrm{~mL} / \mathrm{min}$ & 0.457284833 & 993 & 0.0016 & 0.00078 & 8.822741943 \\
\hline MC6 & $3 \mathrm{~mL} / \mathrm{min}$ & 0.491280942 & 993 & 0.0016 & 0.00078 & 9.31134161 \\
\hline MC7 & $3 \mathrm{~mL} / \mathrm{min}$ & 0.476073521 & 993 & 0.0016 & 0.00078 & 8.751037099 \\
\hline MC3 & $10 \mathrm{~mL} / \mathrm{min}$ & 0.489501275 & 993 & 0.0053 & 0.00078 & 36.64200951 \\
\hline MC7 & $10 \mathrm{~mL} / \mathrm{min}$ & 0.476073521 & 993 & 0.0053 & 0.00078 & 28.98781039 \\
\hline
\end{tabular}


TABLE IV - DETERMINATION OF 20 GAUGE BEAD DIAMETERS FROM MATLAB

\begin{tabular}{|c|c|c|}
\hline $\mathrm{N}$ (10 beads each) & Diameter (Pixels) & Diameter $(\mathrm{mm})$ \\
\hline 1 & 298.53 & 2.688974959 \\
\hline 2 & 303.74 & 2.735903441 \\
\hline 3 & 309.53 & 2.788056206 \\
\hline 4 & 305.44 & 2.751215997 \\
\hline 5 & 310.63 & 2.797964331 \\
\hline MEAN & 305.574 & 2.752422987 \\
\hline
\end{tabular}

TABLE V - DETERMINATION OF 26 GAUGE BEAD DIAMETERS FROM MATLAB

\begin{tabular}{|c|c|c|}
\hline $\mathrm{N}$ (10 beads each) & Diameter (Pixels) & Diameter $(\mathrm{mm})$ \\
\hline 1 & 243.07 & 2.189425329 \\
\hline 2 & 251.86 & 2.268600252 \\
\hline 3 & 241.39 & 2.17429292 \\
\hline 4 & 247.02 & 2.225004504 \\
\hline 5 & 255.54 & 2.301747433 \\
MEAN & 247.776 & 2.231814088 \\
\hline
\end{tabular}




\section{VITA}

Matthew James Robeson was born on August 23, 1991 in Bowling Green, Kentucky. He lived in Bowling Green, Kentucky throughout his early life, until moving to Louisville, Kentucky in the fall of 2010 to pursue an undergraduate degree at the University of Louisville. After graduating with a bachelor's degree in spring 2014, he undertook the Masters of Engineering program in Chemical Engineering. His expertise in computational fluid dynamics was developed through the NSF REU program at Illinois Institute of Technology and various consulting projects through his mentor, Dr. Eric Berson. In the fall of 2015, Matthew will enroll in PhD studies at the Wallace H. Coulter Department of Biomedical Engineering at Georgia Institute of Technology and Emory University in Atlanta, Georgia. 